\title{
Development of the Dynamic Evaluation Model to Significantly Advance Autism Research
}

\author{
Dana Cihelkova
}

Follow this and additional works at: https://researchrepository.wvu.edu/etd

\section{Recommended Citation}

Cihelkova, Dana, "Development of the Dynamic Evaluation Model to Significantly Advance Autism Research" (2014). Graduate Theses, Dissertations, and Problem Reports. 5368.

https://researchrepository.wvu.edu/etd/5368

This Dissertation is protected by copyright and/or related rights. It has been brought to you by the The Research Repository @ WVU with permission from the rights-holder(s). You are free to use this Dissertation in any way that is permitted by the copyright and related rights legislation that applies to your use. For other uses you must obtain permission from the rights-holder(s) directly, unless additional rights are indicated by a Creative Commons license in the record and/ or on the work itself. This Dissertation has been accepted for inclusion in WVU Graduate Theses, Dissertations, and Problem Reports collection by an authorized administrator of The Research Repository @ WVU.

For more information, please contact researchrepository@mail.wvu.edu. 
Development of the Dynamic Evaluation Model to

Significantly Advance Autism Research

\title{
Dana Cihelkova
}

\author{
Dissertation submitted \\ to the College of Education and Human Services \\ at West Virginia University \\ in partial fulfillment of the requirements for the degree of \\ Doctor of Philosophy in \\ Education/Learning, Instructional Design and Technology
}

\author{
Dan Hursh, Ph.D., Chair \\ Reagan Curtis, Ph.D., Chair \\ Elizabeth Juckett, Ph.D., Committee Member \\ Sam Stack, Ph.D., Committee Member \\ Malayna Bernstein, Ph.D., Committee Member \\ Department of Learning Sciences and Human Development
}

\author{
Morgantown, West Virginia \\ 2014
}

Keywords: Autism treatments, evaluation methodological tool, program evaluation Copyright 2014 Dana Cihelkova 


\author{
Abstract \\ Development of the Dynamic Evaluation Model to \\ Significantly Advance Autism Research
}

\title{
Dana Cihelkova
}

In my dissertation effort I suggested new directions in order to significantly advance Autism Research. I moved from identification of the problem (Stagnation of Autism Research) to offering one possible explanation for the problem (Conceptualization of Autism as a Complex Phenomenon) to one possible solution for the problem. I developed the Dynamic Evaluation Model which is a five dimensional evaluation system to (a) effectively evaluate Autism interventions, (b) uncover Autism symptoms dynamics, and (c) identify what child with what symptoms characteristics will benefit the most from what currently available treatments. And finally I tested the prototype of the Dynamic Evaluation Model on evaluation of the Competent Learner Model at The Vista School. Results suggest that the Competent Learner Model is effective in developing in School Community, Play and Leisure, Coping, Social Interaction, Autism Index, Expressive Communication, and Auditory Comprehension in children with Autism. The Competent Learner Model might be an optimal fit for children with Autism who (a) have need to decrease Stereotyped Behavior and (b) are manifesting severe intensity of Stereotyped behavior. 
Copyright (C) 2014 Dana Cihelkova

All Rights Reserved 


\section{Acknowledgements}

I would like to express my deep gratitude, appreciation, respect, and thankfulness toward my mentor, advisor, and teacher, Dan Hursh, Ph.D. My research expedition started with him. I am a former writer and journalist, hence I initially had neither the understanding nor the desire to perform or appreciate research. Dan Hursh helped me to successfully navigate my years in graduate school, including a world of chaos, a hectic life, information overload, and assignments spins. Professor Hursh found and unlocked the hidden locker of my skills, creativity, vision and knowledge so I could explore, while not being overly afraid, new directions and unexplored possibilities in Autism research. Professor Hursh is a man of honor, nobility, goodness and an aristocrat among mentors. He everlastingly impacted me as a person, teacher, researcher and parent. His dexterous, sensitive and intuitive qualities create a remarkable tutor, professor, behaviorist and coach. All I have done in this dissertation and all I will do in my future work would and will be impossible without daily mentoring and preparation from Dan Hursh. He was willing without any reservation to sacrifice his free time, buoyancy and energy to help me better understand myself, the world around me, the complexities of Autism and the intricacy of a researcher's mind, uncertainties, self-doubt and mission. Dr. Hursh never hesitated to provide me with valuable and detailed feedback, (at times) brave comments, encouragement, emotional support, intellectual engagement, and interest, no matter what I did or wanted to do. I am not sure if in my future I will be successful in creating the research methods and/or tools that will assist researchers in Autism and Cancer research to gain profound understanding into these perplexing phenomena. That is my life's mission. If I succeed, this success will belong to Professor Hursh, a person who discovered and unraveled my abilities, personal dimensions and passion toward research.

Along with Dan Hursh is Elizabeth Juckett, Ph.D., a person with whom I began my American educational journey. It started when I took her Novel class as an undergraduate student. Elizabeth Juckett is a rare professor who can inspire, motivate, influence, and encourage her students to achieve more than they thought they could. Professor Juckett supported my educational endeavors emotionally, intellectually, practically, enthusiastically and with unbreakable trust in my aptitude. Exactly this trust helped me to overcome some difficult times. Dr. Juckett always knew when I needed encouragement, reinforcement or compassion. Elizabeth Juckett has for a decade delivered to me the vitality, faith, confidence, commitment, and conviction that I could do what I thought I would like to do. Hence, I did, and the credit clearly belongs to her endurances, métiers and serenity. Besides her high ethical standards and moral influence on me, Professor Elizabeth Juckett had an important impact on my English language skills by facilitating my learning of textual interpretation techniques that I have translated to my Autism research. This came about because I absolutely loved her stimulating, thoughtful and provoking class discussions, in addition to many personal encounters. Our invigorating discussions went from analyzing the subtleties of Brontë novels to the multi-cultural spirals of Cather's My Ántonia to anguish regarding oppressive poverty's impact on the majority of the world population to the densities of Autism's impact on families.

Last but not least in my development as a researcher, scholar, and person firmly stands

Reagan Curtis, Ph.D. His impact has mainly been on my teaching style, because he has modeled 
for me a type of teaching that I consider humanist, active, enjoyable, and energizing while covering abstract subjects such as Statistics, Program Evaluation, Mixed Methods and etc. Professor Curtis is the magician who turned me from a person who disliked statistics and did not believe in statistical methods, processes and purposes into a person who respects statistical apparatuses as the ultimate organizers of the omnipresent data that surround our daily activities. Dr. Curtis is a brilliant teacher, leader, tutor, and academic and personal counselor. He was my academic advisor along with Dr. Hursh for the two years of my master's program and three years of doctoral study. All of these years he encouraged, supported and challenged me to reach my potential while continuously cheering for my new ideas, activities and research projects. Professor Reagan Curtis is a researcher with both a sensitive heart and an exceptional mind. To learn from him and to cooperate with him on any type of research project was always a pleasure, and ultimately an abundant learning experience.

In this way I would like to express to Dan Hursh, Elizabeth Juckett and Reagan Curtis my gratitude, humility, and appreciation for their foundational, formative, influential, and pivotal impact on my graduate studies, personal and professional life.

Thank you all!

In Morgantown, May 15, 2014, West Virginia University

Dana from Prague 


\section{Dedication}

This dissertation is dedicated to General Oldřich Tichý my Grandfather who was my first Teacher. I spend with him five most peaceful, inspiring, enriching, vibrant and heartening years despite of communist oppression we had to daily face. My Grandfather was passing through many adrenalin-laden experiences for a good part of his life. He started as seventeen years old Austro-Hungarian Empire solder in WWI and moved to a high-level intelligence officer during WW II. He was charged with critical missions, some of which put him in a direct line of cooperation with the likes of Generals Patton and Eisenhower. In the post war period after the overthrow of the Czechoslovakia democratic government by communists' dictatorship, my grandfather had to endure a different kind of ordeal by being demoted from General to Private and thrown into a cruel communist jail. Yet, even when having to face not only professional dishonor but also overwhelming personal humiliation, he retained his dignity. I hold a profound respect to him for being able to achieve reconciliation and peace between his inner world and the outside world, regardless whether the outside world was friendly or hostile. Furthermore, I am deeply grateful that he courageously stepped into my troublesome childhood journey and helped me to find the essence of a human survival - hope. 


\section{Table of Contents}

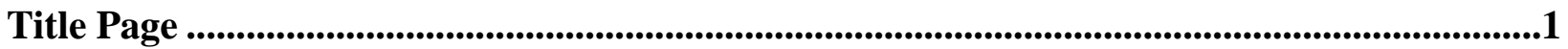

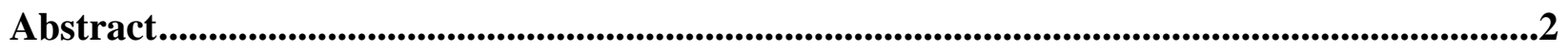

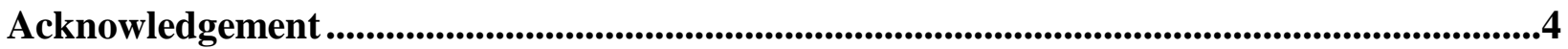

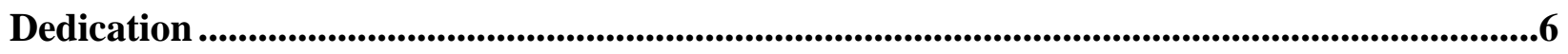

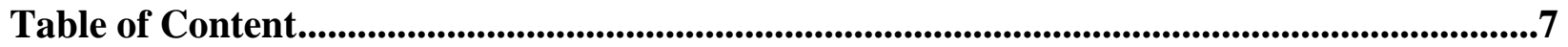

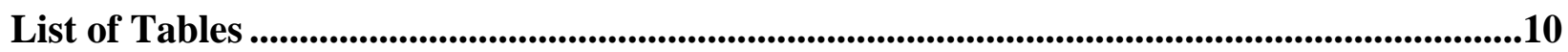

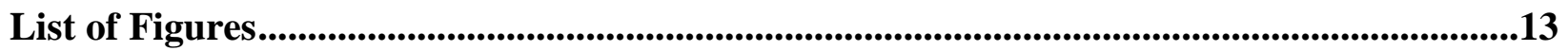

Chapter 1: Problem Identification ...........................................................................................14

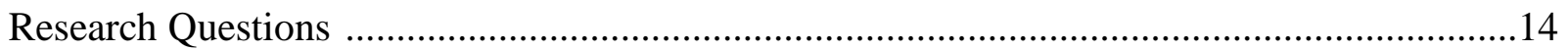

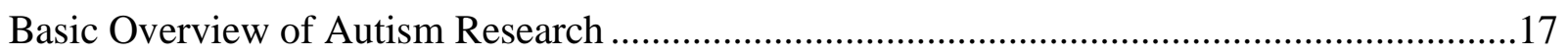

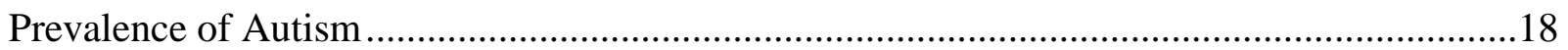

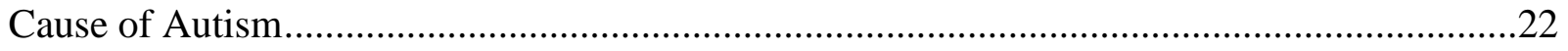

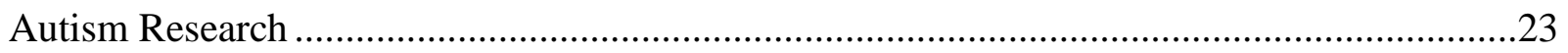

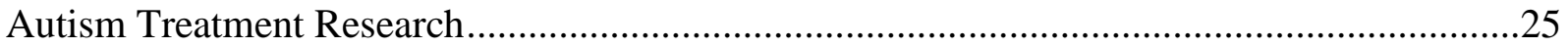

Research Detail of Lovaas Institute ....................................................................28

Method of Systematic Literature Research .................................................................28

Overview of the Lovaas Discrete Trails Treatment ......................................................28

Research Detail of The Koegel Center: Pivotal Response Training ...................................32

Method of Systematic Literature Research ....................................................................32

Overview of the Pivotal Response Training Treatment......................................................32

Research Detail of the Strategic Teaching and Reinforcement System: Verbal Behavior...34

Method of Systematic Literature Research ......................................................................34

Overview of the Strategic Teaching and Reinforcement System Treatment.......................35

Category B: Psychological Based Treatments ..............................................................36

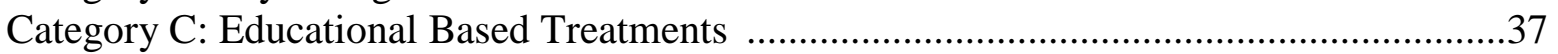

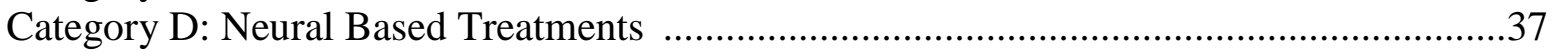

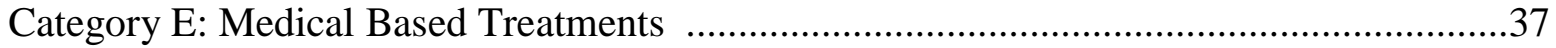

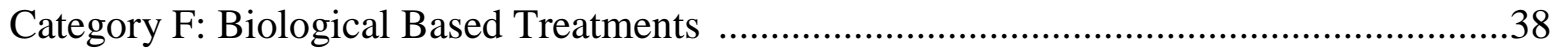

Chapter: 2 Problem Explanation Conceptualization of Autism as Complex Phenomenon.39

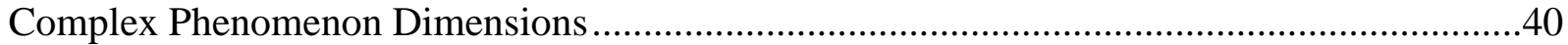

Culture of Evidence versus Culture of Exploration ...........................................................44

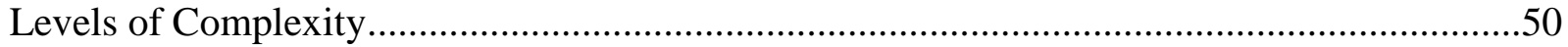

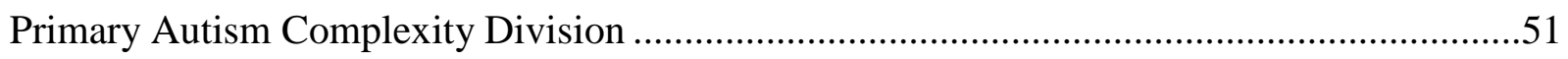

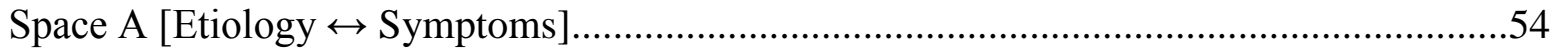

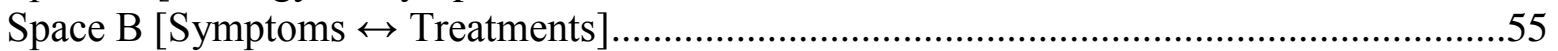

Space C [Treatments $\leftrightarrow$ Treatments Outcomes] ...........................................................56

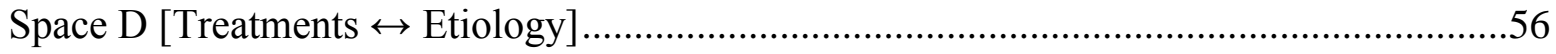

Chapter 3: Problem Solution The Dynamic Evaluation Model...............................................58 
Logic Models vis-à-vis Evaluation of Autism Treatments .................................................60

Theoretical Assumptions of the Dynamic Evaluation Model...............................................62

The Dynamic Evaluation Model ......................................................................................64

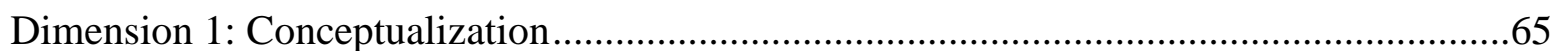

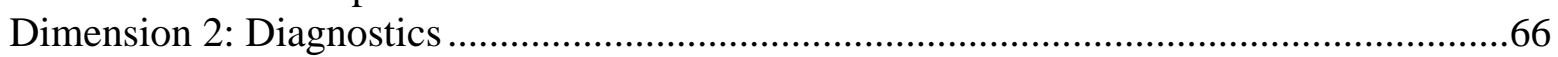

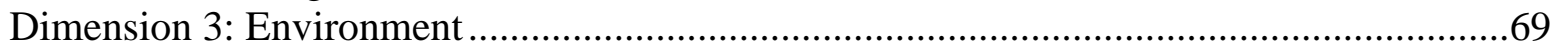

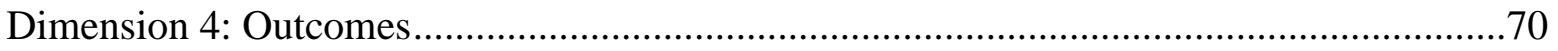

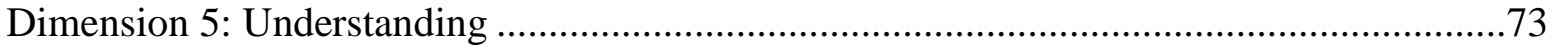

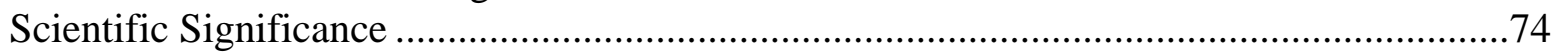

Chapter 4: Prototype of The Dynamic Evaluation Model at The Vista School ......................75

Evaluated Complex Behavioral Program: The Competent Learner Model ............................75

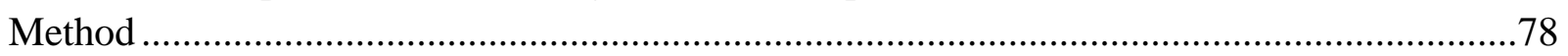

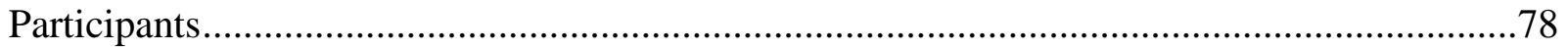

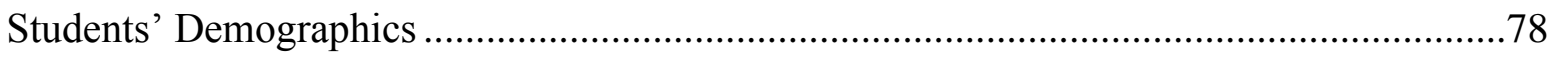

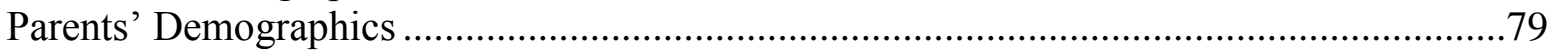

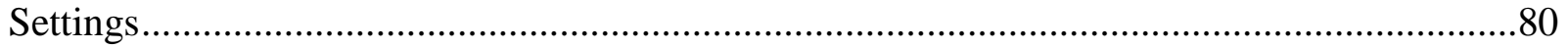

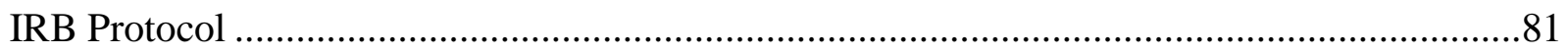

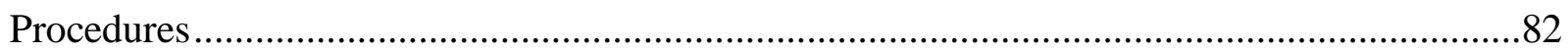

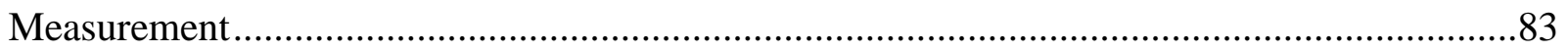

The Vineland Adaptive Behavior- Second Edition ......................................................83

The Gilliam Autism Ratings Scale- Second Edition.......................................................84

The Preschool Language Scales-Fifth Edition..........................................................85

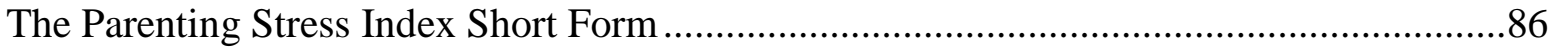

Measurement of Satisfaction with the CLM ultimate Outcomes......................................86

Measurement of Parental and/or Caregivers Satisfaction with The Vista School ...............87

Measurement of Administration and Staff Satisfaction with the CLM Ultimate Outcomes .87

Measurement of Teachers, Administration and/or Staff Satisfaction with Other Components

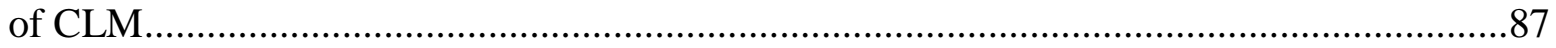

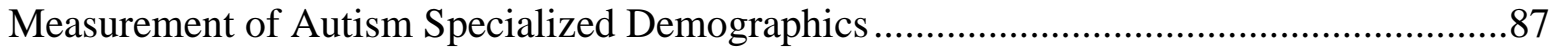

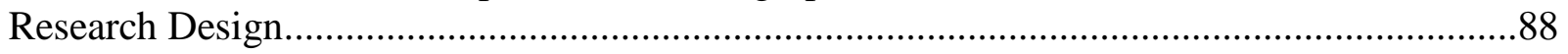

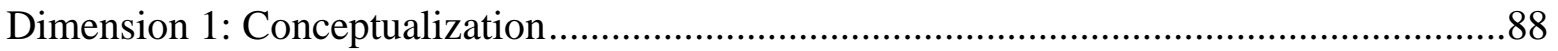

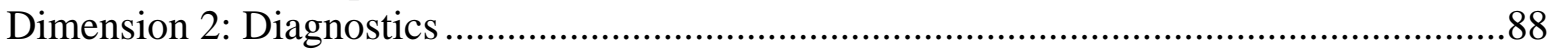

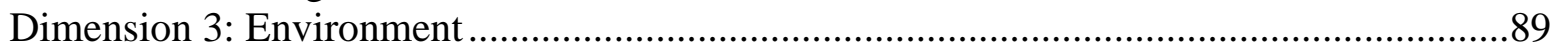

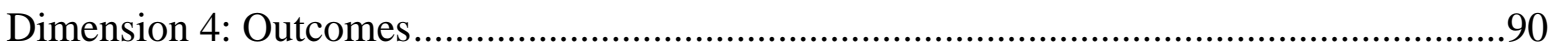

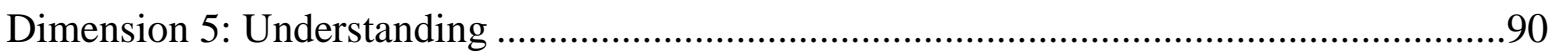

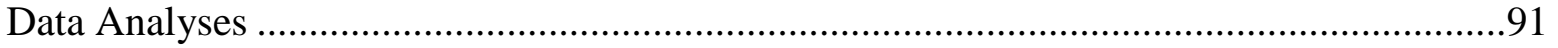

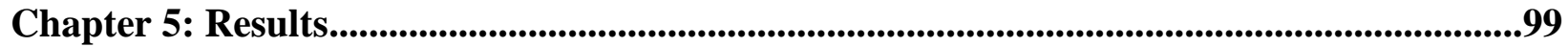

Results from Dimension 1: Conceptualization ........................................................99

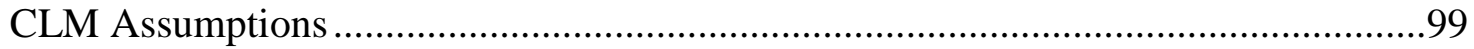

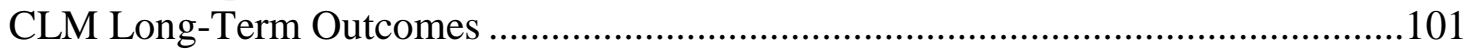

CLM Ultimate Outcomes............................................................................... 102

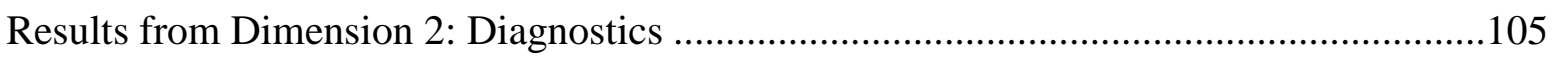

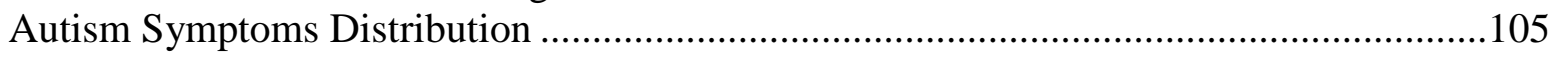




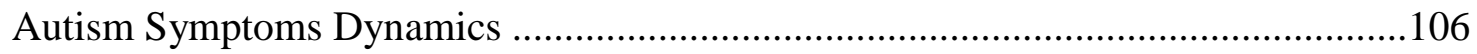

Autism Symptoms and Treatment Outcomes Dynamics .......................................107

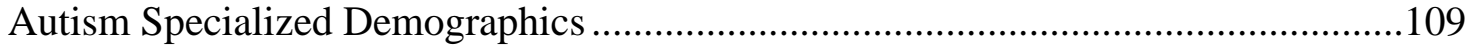

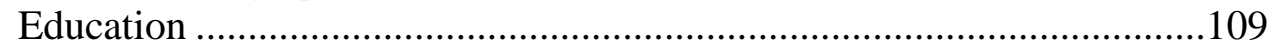

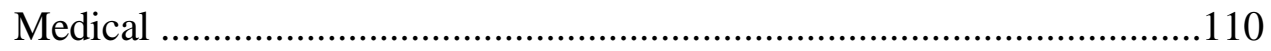

Other Treatments for Autism ..........................................................110

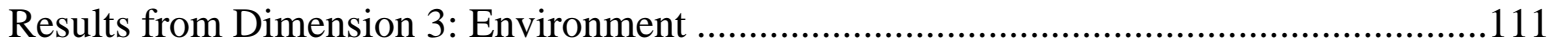

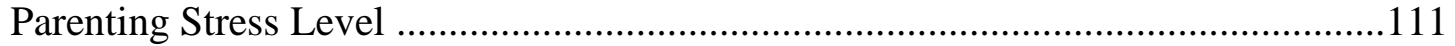

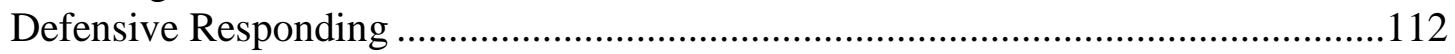

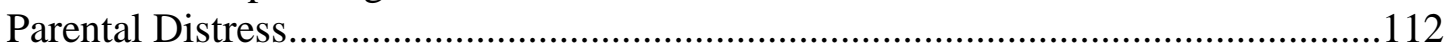

Parental-Child Dysfunctional Interactions...................................................... 112

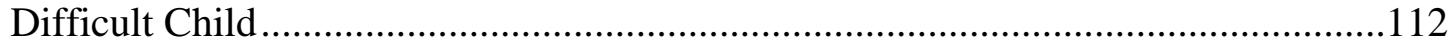

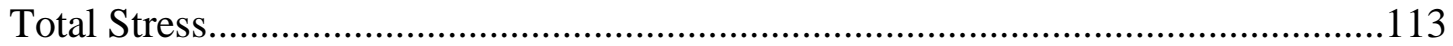

Satisfaction with the CLM Outcomes ....................................................................... 113

Parents Satisfaction with CLM Ultimate Outcomes .................................................113

Parents Satisfaction with The Vista School ...........................................................114

Administration and Staff Satisfaction with CLM Ultimate Outcomes ....................117

Results from Dimension 4: Outcomes ........................................................................125

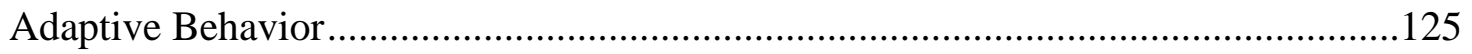

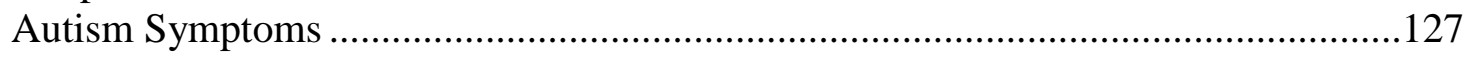

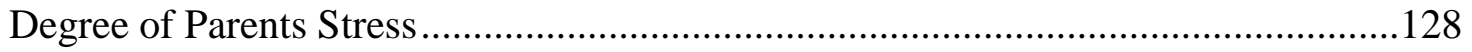

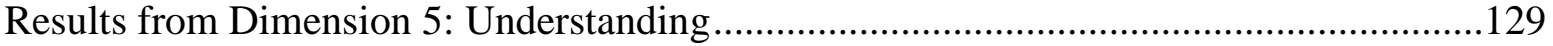

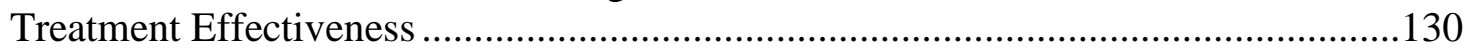

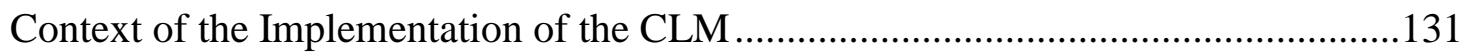

Identification of Who Benefits the Most from the CLM .......................................131

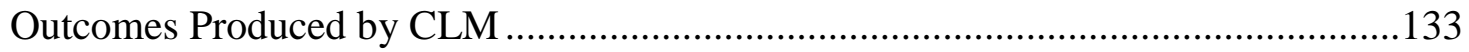

Chapter 6: Discussion and Conclusion ............................................................................134

Research Question 1: Conceptualization .....................................................................134

Research Question 2: DEM ......................................................................................138

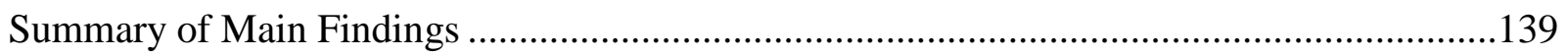

Limitation of the Model ................................................................................................140

Research Question 3 Effect of the CLM.......................................................................142

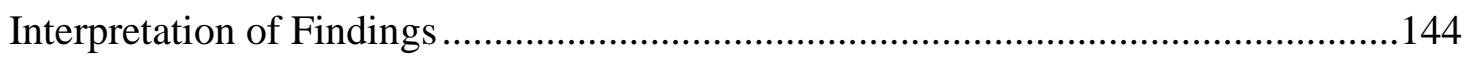

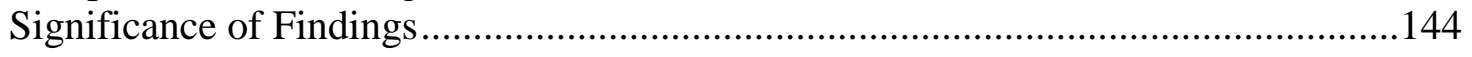

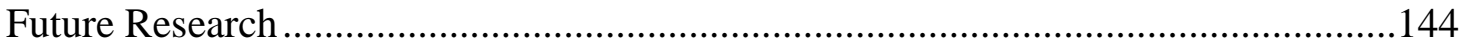

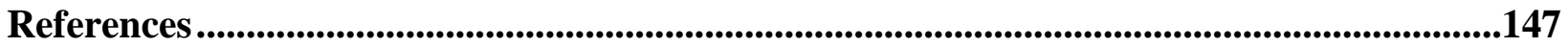

Appendix 1 ....................................................................................................................................................160

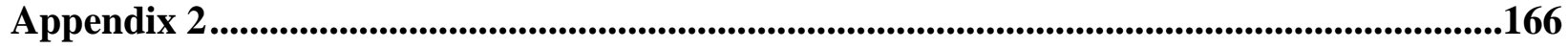

Glossary of New Terms ............................................................................................................................190

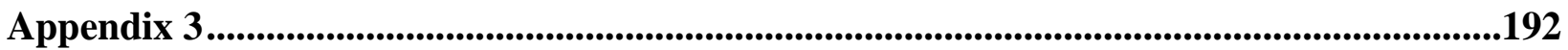




\section{List of Tables}

\begin{tabular}{|c|c|c|}
\hline Table & Name & Page \\
\hline 1.1 & A Sample Representation of Types of Research Methodology & 27 \\
\hline 3.1 & The Gilliam Autism Rating Scales: Stereotyped Behaviors & 68 \\
\hline 3.2 & The Gilliam Autism Rating Scales: Communication & 68 \\
\hline 3.3 & The Gilliam Autism Rating Scales: Social Interaction & 69 \\
\hline 3.4 & The Vineland Adaptive Behavior Preschool Language Scales Composites & 72 \\
\hline 5.1 & $\begin{array}{l}\text { Autism Symptoms Distribution at The Vista School Simple General } \\
\text { Tabulation }\end{array}$ & 106 \\
\hline 5.2 & Autism Symptoms Dynamics at The Vista School & 106 \\
\hline 5.3 & $\begin{array}{l}\text { Dynamics between the Autism Symptoms and Outcomes at The Vista } \\
\text { School }\end{array}$ & 108 \\
\hline 5.4 & Autism Demographics: Parents Education-Major at The Vista School & 109 \\
\hline 5.5 & Parenting Stress Index at The Vista School & 113 \\
\hline 5.6 & $\begin{array}{l}\text { Likert-type Survey Item: Parents and/or caregivers satisfaction with the } \\
\text { CLM Ultimate Outcomes }\end{array}$ & 114 \\
\hline 5.7 & Likert-type Survey Item: Parents' Satisfaction with The Vista School & 115 \\
\hline 5.8 & $\begin{array}{l}\text { Sample of Parent's Identification of the Strongest Components of The } \\
\text { Vista School }\end{array}$ & 116 \\
\hline 5.9 & $\begin{array}{l}\text { Sample of Parents' Suggestions for improvement and/or changes at The } \\
\text { Vista School }\end{array}$ & 117 \\
\hline 5.10 & $\begin{array}{l}\text { Likert-type Survey Item: Teachers' assessment of the CLM Ultimate } \\
\text { Outcomes for SOME Students }\end{array}$ & 118 \\
\hline 5.11 & $\begin{array}{l}\text { Likert-type Survey Item: Teachers' assessment of the CLM Ultimate } \\
\text { Outcomes for ALL Students }\end{array}$ & 119 \\
\hline 5.12 & $\begin{array}{l}\text { Likert-type Survey Item: Teachers, administration and staff satisfaction } \\
\text { with other components of CLM }\end{array}$ & 121 \\
\hline 5.13 & $\begin{array}{l}\text { Sample of Teachers and Staff identification of the strongest components } \\
\text { of the CL }\end{array}$ & 123 \\
\hline 5.14 & Sample of Teachers and Staff suggestions for improvement of the CLM & 124 \\
\hline
\end{tabular}


5.15 Pair Sample Test for The Vineland Adaptive Behavior Scales 126

5.16 Paired Sample Test for The Gilliam Autism Rating Scales for Autistic 127 Symptoms

5.17 Pair Sample Test for Auditory and Expressive Comprehension of The 128 Preschool Language Rating Scales

5.18 Paired Sample Test for The Parenting Stress Index 129

5.19 Comparison Parents and Teachers satisfaction with Ultimate Outcomes 133

A1/1 Examination of the Normality Assumption: Kolomogorov-Smirnov Test 160

A2/1 Category A: Comprehensive Evaluation of Autism Behavioral Based 166 Treatment

A2/2 Category B: Comprehensive Evaluation of Autism Psychological Based 167 Treatment

A2/3 Category C: Comprehensive Evaluation of Autism Educational Based

Treatment

A2/4 Category D: Comprehensive Evaluation of Autism Neural Based

Treatment

A2/5 Category E: Comprehensive Evaluation of Autism Medical Based

Treatment

A2/6 Category F: Comprehensive Evaluation of Autism Biological Based

167

Treatment

A2/7 Summary of Research Regarding Lovaas' DTT 169

A2/8 Summary of Research Regarding: Pivotal Response Training 178

A2/9 Summary of Research Regarding: The Strategic Teaching and 189

Reinforcement System: Verbal Behavior program

A2/10 Glossary of New Terms 190

A3/1 Compete Survey Items for Parents and/or Caregivers 192

A3/2 Compete Survey Items for Administration and Staff and/or Caregivers 193

A3/3 Transcription of Parents Answers: Strongest Component of The Vista 195 School

A3/4 Transcription of Parents Answers: In What Ways can be The Vista School 198 improved 
A3/5 Transcription of Staff Answers: Strongest Components of the CLM 201

A3/6 Transcription of Staff Answers: In What Ways CLM can be Improved 203

A3/7 Booklet for the CLM Developer: Plan of the Evaluation and Pilot Surveys 208 


\section{List of Figures}

\begin{tabular}{clc}
\hline Figure & & \multicolumn{1}{c}{ Name } \\
\hline 2.1 & Primary Autism Complexity Division & 52 \\
2.2 & Primary Autism Complexity Division & 53 \\
4.1 & Normal Q-Q Plot for distribution D18 (Motor Skills) & 95 \\
4.2 & Normal Q-Q Plot for distribution D19 (Gilliam Autism Index) & 96 \\
4.3 & Normal Q-Q Plot for distribution D23 (Auditory Comprehension) & 96 \\
4.4 & Normal Q-Q Plot for distribution D29 (Total Stress) & 97 \\
5.1 & Autism Index Scores Before and After CLM Treatment & 132 \\
A1/2 & Examination of the Normality Assumption: Normal Q-Q Plots & 161 \\
\hline
\end{tabular}




\section{Chapter 1: Problem Identification \\ - Stagnation in Autism Research -}

Autism Spectrum Disorder is a phenomenon that has been extensively studied over the last seventy years. However, these studies have not been sufficient to develop a profound understanding of the complex phenomenon that is Autism. Right now researchers and practitioners are at the stage of understanding where they know enough to recognize the complexity of the characteristics of Autism but do not understand the dynamics between single factors and the sum of the factors that contribute to Autism. None of these studies have uncovered the cause or causes of Autism. None of these studies conveyed understanding of the increasing prevalence of Autism or uncovered true prevalence patterns. None of these studies developed a reliable evaluation system to identify effective treatments for the considerable variability in symptoms among children with Autism. None of these studies explained the complexity around the phenomenon of differential responsiveness (50\% of children with Autism may benefit from the treatment while $50 \%$ do not). None of these studies provide insights into the dynamics of Autism symptomology, and none are able to even agree on a definition of Autism. Therefore, the purpose of the methodological study in this dissertation is (a) to suggest a new direction by posing and exploring three research questions, and (b) to develop a methodological tool to significantly advance Autism Research.

\section{Research Question 1:}

How can we conceptualize Autism research, Autism per se, and evidence for the investigation of complex phenomena to deepen contemporary understanding of Autism? 


\section{Research Question 2:}

What is the best methodological tool to accelerate the assembly of nationwide evidence-based Autism treatments in order to:

(a) Develop a three dimensional matrix of child Autism symptoms, treatments constellations, and behavior outcomes.

(b) Uncover cluster distributions of autism symptoms

(c) Determine in what terms the treatment is effective

(d) Determine what is the context of the treatment implementation

(e) Uncover who benefits the most from what particular intervention

(f) Uncover what programs are able to produce what outcomes

(g) Understand the distribution of Autism symptoms

(h) Uncover the dynamics of Autism symptomology

(i) Understand the dynamics of Autism symptomology

(j) Contribute to the explanation of the phenomenon of differential responsiveness

(k) Understand the impact of the environment in the treatment process and outcomes

(1) Understand the impact of the treatment process and outcomes on the environment

(m) Understand the impact of overall contextual factors

\section{Research Question 3:}

What is the effect of the Competent Learner Model on adaptive behavior, language development, and Autism Symptoms of children with Autism Spectrum Disorder? What is the effect of the Competent Learner Model on parental stress of parents of children with Autism? 
In the current chapter, I briefly overview the Autism Spectrum Disorder and the intricacy of the prevalence of Autism. I suggest that consistency in prevalence be achieved by adopting epidemiology measures and developing the Autism Incidence Rate, the Autism Cumulative Incidence, the Autism Point Prevalence, and the Autism Period Prevalence. I review currently available treatments and show that: (a) there is no methodological tool to systematically and comprehensively evaluate programs/treatments for children with Autism, and (b) there is no comprehensive evaluation of an entire program(s) for treatments for Autism.

In chapter 2, I explore research question 1 (How can we conceptualize Autism research, Autism per se, evidence for investigation of complex phenomena to deepen contemporary understanding of Autism?) and suggest the conceptualization of Autism as a complex phenomenon. I develop and describe a definition of Autism Dynamics. I create and describe a method (Primary Autism Complexity Division) to break down a large complexity block leading to contradictory and controversial results in Autism research. I coin and explain the meaning of several new terms: Complex Phenomenon Dimension, Complex Phenomenon Paradox, Simpleness, Categorization of Effectiveness, Primary Autism Symptoms, Individual Autism Symptoms. And last I suggest classification and division of monolithic Autism Research into specific subfields of Autism Etiology Research, Autism Treatment Research, Autism Epidemiology Research, Autism Family Research and Autism Prevention research.

In chapter 3, I answer research question 2 (What methodological tool can accelerate the assembly of nationwide evidence- based Autism treatments) by creating and describing a fivedimensional program evaluation model to handle the enormous variability of Autism symptoms and to provide a type of information that is not currently available. 
In chapter 4, I identify the methods, participants, location, procedures, measurements and research design to answer research question 3 (What is the effect of the Competent Learner Model on adaptive behavior, language development, and Autism symptoms of children with Autism Spectrum Disorder? What is the effect of the Competent Learner Model on parental stress for parents of children with Autism?)

In chapter 5, I present specific results for research question 3 generated by application of the Dynamic Evaluation Model.

In chapter 6, I discuss the results in broad context and briefly consider the utility, accessibility, limitations and possible improvement of the Dynamic Evaluation Model.

\section{Basic Overview of Autism Spectrum Disorder}

Autism Spectrum Disorder is understood as a developmental disorder that is predominately characterized by more or less severe deficits in communication skills and social interactions, self-absorption, and repetition of certain behavioral patterns. The clinical signs of Autism are manifested around 3 years old, and the heterogeneity of Autism is manifested by clinical phenotype, severity, and frequency of symptoms (Levy, Mandell, \& Schultz, 2009). Autism is perhaps one of the most complex developmental disorders for which a single straightforward definition does not exist. Definitions range from Autism being a psychiatric disorder, to a neurobiological disorder, to a pervasive developmental disorder, to a neurodevelopmental syndrome. Specifically, at the onset of Autism research, Kanner defined Autism as a psychiatric disorder characterized by an aloof child (Steyaert, De La Marche, 2008). The National Institute of Child Health and Human Development defined Autism as a neurobiological disorder of development (National Inst. of Child Health and Human Development, 2005). Some researchers suggest that the Autism spectrum includes neuro-developmental syndromes that have 
a strong heritability factor (Baron-Cohen, 2004; Gurkan, \& Hagerman, 2012; Levy, et al., 2009; Tchaconas, \& Adesman, 2013). According to the American Psychiatric Association, Autism Spectrum Disorder is categorized as a Pervasive Developmental Disorder.

We are basically moving from the speculation that Autism is an abnormal behavioral pattern or mental illness, to a disorder of the nervous system, to delay in development impacting multiple functions such as communication or socialization, to impairment of brain functions impacting learning ability. Because the definitions of Autism substantially differ, this impacts the ways in which we research this phenomenon and the ways we develop treatments for Autism. It leads us into vast variability that may be described as a chaos in our understanding. It does not mean that we need to be terrified of this situation. Rather, we can perceive the chaos in Autism research as a lack of recognition of complex interactions among parts of the phenomenon. I suggest that the chaos in Autism research is only a temporary occurrence due to lack of understanding of the enormous variability of the interacting factors relevant to Autism. Once we are able to see all the connections and interactions and thus the reoccurring patterns, we will resolve the chaos in Autism research. In order to significantly progress in Autism research, we must precisely define the problem. I suggest that Autism research stagnates or rather rotates around the same type of thinking and thus the same type of research questions yield similar results. Consequently, no progress in our understanding can be made perhaps due to a fundamental lack of conceptualization of the complex phenomenon per se. Autism is a complex phenomenon, for which the fundamental blueprint is enormous variability.

\section{Prevalence of Autism}

Even something relatively simple such as prevalence is an intricate problem in Autism research. The prevalence is counted differently in different times, and different sources present 
different numbers for prevalence. The estimates of the prevalence of Autism vary, but overall the prevalence of Autism has substantially increased from 1960. The prevalence in the US between 1960 and 1980 increased from 5 to 72 cases per 10,000 children (Levy et al., 2009). According to Wing and Potter, (2002), however, in the fifties, sixties, seventies, and even eighties, the prevalence of Autism was only 2 or 4 per 10,000 children; Autism was considered rare until it substantially increased after 1990 (Wing \& Potter, 2002). While it is difficult to determine the true trend of prevalence, it is clear that Autism's prevalence is rapidly increasing. According to the Centers for Disease Control and Prevention (2009), 1 in 110 children is classified as having an Autism Spectrum Disorder. Forty years ago it was 1 child in 2000 (Dicker, 2013). Now the question is why is prevalence increasing? Is it truly increasing? Why do we have different estimations of prevalence?

Levy et al., (2009) speculate that the different estimations were influenced by screening, case-conformation strategies, and sample sizes. Williams, Higgins, and Brayne, (2006) speculate that the prevalence numbers of Autism differ due to the changing diagnostic criteria, the age of the children screened, and the geographical location. Wing and Potter (2002) agree with Williams et al. (2006) that prevalence counts differ due to changing diagnostic criteria and add that increasing prevalence may be due to (a) the fact that different studies used different prevalence system counts, (b) the increasing knowledge among parents, (c) better recognition among professional workers, (d) development of the Autism concept, (e) establishing specialized services, and last but not least (f) the possibility that there is a real increase in the prevalence of Autism.

The facts that we do not know the true prevalence and that we do not understand the reasons behind increasing prevalence have driven global research to a place where we cannot 
make progress in Autism research. Essentially the problem with Autism prevalence boils down to three possible things: either we have a counting problem or Autism is truly increasing or both. However, in order to advance Autism research, it is necessary to uncover the reason(s) because we are depriving our research from valuable connections and possible directions to advance knowledge about Autism. For instance, if Autism as a condition is truly increasing, then we can develop research that would uncover what changed in society around the time Autism started increasing. Did society go through some chemical changes in its products, were new drugs introduced, birth control composition changed, food composition changed, and so on? To know whether Autism is truly increasing is absolutely essential for substantial progress because it would suggest an environmental impact as opposed to genetic influence. By the same token, we need to have a stable and reliable counting system to uncover the trajectory of Autism's prevalence and to see patterns in the changes.

I suggest that one of the reasons we do not have a reliable prevalence count is that we have not created a reliable system for counting Autism cases. There are many variables to consider: are we taking prevalence in each state, all of the states, each year, in certain periods, at what age, all the persons with Autism, etc.? It appears that Autism epidemiology research needs to develop a counting system that will be consistent across at least a century so that we can see accurate patterns of Autism's prevalence.

I suggest that for this to happen it is necessary to determine consistent diagnostic criteria rather than considerably changing diagnostic criteria from one edition of the Diagnostic and Statistical Manual of Mental Disorders (DSM) to another. Specifically, in the DSM-I, Autism did not have any diagnostic criteria, instead was classified as childhood schizophrenia (American Psychiatric Association, 1952). In DSM-II, Autism was still categorized as childhood 
schizophrenia, but some of the behaviors were described as "autistic, atypical, and withdrawn behavior" (American Psychiatric Association, 1968). In DSM-III, Autism had its own category, which was called Infantile Autism and had six sub-categories. In order to be diagnosed with Autism, the individual had to have each and all of the characteristics (American Psychiatric Association, 1980). In DSM-III-R, the diagnostic criteria were stipulated to be specific behavior that is observable and interferes with individual development (American Psychiatric Association, 1987). In DSM-IV several categories and subtypes of pervasive developmental disorders were added. Sixteen symptoms were described but only six needed to be exhibited for a diagnosis of Autism Spectrum Disorder (American Psychiatric Association, 1994). In DSM-V, we are moving back toward more conservative diagnostic criteria, thus a person can be diagnosed with Autism only if he/she meets all indicators of impairment in (a) social interaction, (b) communication, and (c) at minimum, two signs of repetitive behavior. In addition, Asperger's Syndrome and Pervasive Developmental Disorder-Not Otherwise Specified were removed (American Psychiatric Association, 2013).

It seems that from gaining more knowledge and some understanding of Autism conditions, the American Psychiatric Association was adjusting the diagnostic criteria. Consequently, from 1952 until now, the diagnostic criteria are constantly changing. This suggests that we are lost in the very nature of this condition - complexity; hence we are moving back and forth with diagnostics creating unstable ground for the development of an Autism prevalence system. Obviously we are moving away from a profound understanding of Autism. It is simple: if we cannot determine consistent diagnostic criteria, then we cannot develop or adopt a consistent system for counting the frequency of Autism occurrences. Without assessment of the 
frequency of Autism occurrences, we will never know whether Autism is truly increasing or we are simply more aware of Autism.

For instance, according to Coggon, Rose, and Barker (1997) epidemiology can be defined according to several measures. We can adopt these general epidemiology measures and develop the Autism Incidence Rate ${ }^{1}$, the Autism Cumulative Incidence ${ }^{2}$, the Autism Point Prevalence ${ }^{3}$, and the Autism Period Prevalence ${ }^{4}$. Indeed, all these measures are more or less already taken. In addition, it is very difficult to take these measures, particularly the incidence measure, in such a chronic condition as Autism (Coggon et al., 1997). Still, in order to gain a true insight into whether or not the Autism Condition is truly increasing or we are becoming more aware of Autism, we need a reliable and multifaceted counting system for the prevalence of Autism across time and locations.

\section{Cause}

The cause of the Autism Spectrum Disorder is unknown. And as with everything in Autism Research, there are many speculations about cause (Tchaconas \& Adesman, 2013). However, researchers have worked toward testing some hypotheses about the cause of Autism. According Williams and Williams (2011) there are three main hypotheses:

(a) Autism is caused by the genetics of inheritance

(b) Autism is caused by neuropathology

(c) Autism is caused by Opioid Excess

\footnotetext{
${ }^{1}$ The Incidence Autism Rate: the rate of Autism where new cases are counted per person-year.

2 The Cumulative Autism Incidence: in a specified time period the total number of new Autism cases.

3 The Point Autism Prevalence: Autism population that had the condition at a specific single point in time.

4 The Period Autism Prevalence: the proportion of the population that had Autism at any time within a specific time period.
} 
Some researchers targeting genetic heritability propose that the possible cause of Autism is maternal immune response to a prenatal infection (Tchaconas, \& Adesman, 2013). Other genetics researchers suggest that altered immune responses in the individuals with Autism cause the Autism (Tchaconas, \& Adesman, 2013). Herman (2006) proposes that because there is a 4 to 1 male majority in Autism patients, perhaps there is a sex-related genetic influence. Zhao et al. (2007) speculate that Autism can be passed on by mothers who do not necessarily display Autism themselves but who have a 50 percent risk for Autism development in their children (Zhao et al., 2007). In sum, it seems that more than one genetic system is responsible for Autism but the fact is that the real genetic mechanism behind Autism is unknown (Folstein, 1999).

Research regarding neuropathology indicates that children with Autism tend to have larger brains (Folstein, 1999). Nonetheless, children with Autism are not born with a larger head; rather it starts to grow between the ages of $2-12$, and the brain volume is larger than the normal population's as well (Aylward, Minshew, \&Field, 2001). Children with Autism suffer from seizures and epileptiform discharges. Specifically, 46 percent had seizures and 20 percent epileptiform discharges (Hughes \& Melyn, 2005).

The Opioid Excess Theory suggests that the cause of Autism is due to metabolic disorders. Particularly the opioid peptides (formed via metabolism of gluten and casein) pass through an abnormally penetrable intestinal membrane and in consequence affect neurotransmission via binding with opioid receptors (Millward, Ferriter, Calver, \& Connel-Jones, 2008).

\section{Autism Research}

Autism research started developing slowly after 1943. Autism was first conceptualized and behaviorally described as an obsession with the same objects, regression in verbal ability, temper tantrums, problematic attachment, exceptional memorization ability, repetitive action, 
lack of eye contact etc., by Leo Kanner, who described 11 cases of children with Autism (Kanner, 1943). Today, we are investing millions of dollars from federal, public, or private sectors to sponsor Autism research. For instance, the National Institutes of Health sponsored \$22 million of Autism research in 1997, and in 2006 that had increased to $\$ 108$ million (Amaral, 2011). Currently, the National Institutes of Health sponsors 169 million dollars of Autism Research (Autism Speaks, 2011).

During the past ten years, Autism Spectrum Disorders have received much societal and media attention (Dicker, 2013). The number of articles published during these ten years is about 3,700 (Volkmar, Lord, Bailey, \& Schultz, 2004). According to Autism Speaks, on the average Autism costs a family about 6000 dollars per year. Autism now affects 1 in 88 children and 1 in 54 boys (Autism Speaks, 2011). Researchers from different disciplines have been working very hard around the globe for 71 years. Yet we neither understand what is Autism or is not, nor do we understand the cause of Autism. Thus we do not have a cure and obviously we cannot even start research toward prevention of Autism.

While we have prolific Autism research, it also produces high levels of controversy and confusion among teachers and parents (Tchaconas \& Adesman, 2013). Perhaps, the best-known controversy in Autism Research is one that is called by Dennis Flaherty "the most damaging medical hoax of the last 100 years." (1302) Dr. Andrew Wakefield, a gastroenterologist, described Autism as a "regressive autism-enterocolitis syndrome" that is caused by measles, mumps or rubella (MMR) vaccination (Flaherty, 2011). This led the public into severe distrust of public health vaccination and consequently into a public health crisis (Flaherty, 2011).

Other controversies have developed around treatments for Autism. For example, the Holding Therapy, Megavitamin Therapy, or Facilitated Communication are on the one hand very 
popular treatments but on the other hand are very controversial (Romanczyk, 1999). Schreibman (2008) considers many treatments as ineffective or unproven or limited, such as Facilitated Communication, Option Therapy, Sensory-based Treatments, Nutritional Treatments, or Pharmacological Treatments. When the treatments are lacking objective evaluation, then testimonials, emotions, and wishful desire can lead society into wrongly trusting some of the treatments (Schreibman, 2008).

\section{Autism Treatment Research}

Autism Spectrum Disorder is a complex disorder for which the etiology is unknown. While we do not know and/or understand the etiology of Autism, we are developing assortments of treatments that are more or less effective and are more or less evaluated. Schreibman, (2008) argued that the fact that we do not have the "cure" for Autism results in the situation where currently there are hundreds of treatments available. Autism treatments can be divided into six general categories (Williams \& Williams, 2011).

Category A: Behavioral Based Treatment (ABA)

Category B: Psychological Based Treatments (Relational)

Category C: Educational Based Treatments (Behavioral)

Category D: Neural Based Treatments (Brain)

Category E: Medical Based Treatments (Pharmacological)

Category F: Biological Based Treatments (Nutritional

Perhaps the most prolific treatments are the behavioral treatments based on Applied Behavioral Analysis.

Williams and Williams (2011) identified the following behavioral treatments as the most effective. I searched whether or not these treatments for Autism underwent comprehensive 
program evaluation and found no comprehensive program evaluation of an entire program for any these treatments (see appendix A2, table A2/1).

A1. The Lovaas Institute: Discrete Trials Training

A2. The Koegel Center: Pivotal Response Training

A3. Strategic Teaching and Reinforcement System: Verbal Behavior

A4. Project Data: School-Based Inclusion Model

A5. May Institute: System of Care

A6. New England Center for Children: Teaching Independence evaluation

A7. Princeton Child Development Institute: Across the Lifespan

A8. Judge Rotenberg Center: Zero Exclusion

In addition to Williams and Williams (2011), Odom et al. (2010), identify other Applied Behavior Analysis based treatments, and again I searched whether or not these treatments for Autism underwent comprehensive program evaluation and found no comprehensive program evaluation of an entire program for any of them (see appendix A2, table A2/1).

A9. Autism Partnership Seal Beach

A10: Center for Autism and Related Disorders (CARD) evaluation

A11: Alpine Learning Group Paramus

A12: Eden Institute Princeton - any comprehensive program evaluation

A13: Douglass Developmental Disabilities Center

A14: Institute for Child Development: SUNY

A15: Pyramid Approach to Education

A16: Strategies for Teaching based on Autism Research (STAR)

A17: Summit Academy

A18: Therapeutic Pathways

A19: Valley Program

A20: Children's Toddler School

A21: Walden Model

A22: Preschoolers and Parents (LEAP) 
To examine a cross-section detail of the research activity, I assessed a purposeful sample of the three most prominent behavioral interventions:

A1. The Lovaas Institute: Discrete Trials Training

A2. The Koegel Center: Pivotal Response Training

A3. Strategic Teaching and Reinforcement System: Verbal Behavior

Specifically, I reviewed what type of research was completed in terms of the types of studies, types of research methodology (see summary table below), overall learning (conclusion), and whether or not these studies (a) demonstrated how each of the program components produced the changes in behavior, (b) provided detailed symptoms descriptions of types and intensity for participants and (c) yielded results connected to our understanding of Autism as a complex phenomenon per se. Consequently, I identified the particular articles, empirical studies, reviews, books, and/or dissertations to generate an overall picture of what has been accomplished and what we have learned from Autism treatment research.

Table 1.1

A Sample Representation of Types of Research Methodology

\begin{tabular}{|c|c|c|c|}
\hline Research Type & $\begin{array}{c}\text { Discrete Trials } \\
\%\end{array}$ & $\begin{array}{c}\text { Pivotal Response } \\
\text { Training } \\
\%\end{array}$ & $\begin{array}{c}\text { Verbal Behavior } \\
\#\end{array}$ \\
\hline $\begin{array}{l}\text { Single Case Experimental } \\
\text { Design }\end{array}$ & 18 & 49 & - \\
\hline Qualitative Methodology & 0 & 0 & - \\
\hline Meta-analysis & 5 & - & - \\
\hline Randomized trial & 5 & & \\
\hline Conceptualization papers & 14 & 5 & 1 \\
\hline Methodological criticisms & 9 & & - \\
\hline Position papers & 14 & 8 & \\
\hline $\begin{array}{l}\text { Book reviews, conference } \\
\text { reports, descriptive reports } \\
\text { addressing practical }\end{array}$ & 12 & 29 & 1 \\
\hline $\begin{array}{l}\text { Group } \\
\text { comparisons/Quantitative }\end{array}$ & 23 & 3 & 1 \\
\hline Dissertation & - & 6 & \\
\hline
\end{tabular}




\section{Subcategory A1: The Lovaas Institute: Intensive Early Intervention - Discrete Trial Training}

Method of Systematic Literature Research. I used the two sets of descriptors: 1) "Discrete Trials Training and Autism Treatment or Intensive Early Intervention or Lovaas Institute" which yielded 4824 publications results. Therefore, I narrowed down the research by changing one of the Boolean operators and a descriptor: 2) "Discrete Trials Training and Autism Treatment and Early Intensive Behavioral Intervention or Lovaas Institute” yielded 33 publications (of which a considerable number were duplicates), all of which I have inspected to understand what kind of research was completed regarding this specific treatment program for Autism Spectrum Disorder.

I used the second set of descriptors with the following databases: Education Research Complete, which yielded 11 articles. ERIC yielded 5 publications, Health Science Sources: Consumer Edition yielded 0 articles, Health Science: Nursing/Academic Edition yielded 2 articles, International Pharmacological Abstracts yielded 0 articles, MEDLINE yielded 6 articles, PsycArticles yielded 0 articles and the PsycINFO yielded 14 articles. The articles regarding the Lovaas Institute (Discrete Trials Training) were published in an assortment of different publications between 1971 and 2013.

\section{Overview of the Lovaas' Discrete Trials Treatment}

Discrete Trial Training is considered one of the most studied behavioral treatments based on Applied Behavior Analysis (Smith, 2001). Discrete Trial Training is a set of short instructions commonly (but not exclusively) implemented by a trained behavioral therapist. Each Discrete Trial has 5 steps of cue, prompt, response, consequence, and intertrial interval (Smith, 2001). In step 1 (cue) the teacher (or parent) asks a child questions or gives some 
instruction to do something. In step 2 (prompt) the teacher helps the child to correctly respond. In step 3 (response) the child answers either correctly or not. In step 4 (consequence) the teacher either reinforces the correct answer or says "NO" to an incorrect answer. In step 5 (intertrial interval) the teacher provides about 5 seconds break and starts again with step 1.

According to the program intensity, the child with Autism can experience up to several hours of Discrete Trial instructions (Smith, 2001). It is strongly suggested that the child with Autism receive more than 40 hours of one-to-one trained therapist treatment per week (Lovaas, 1987). Discrete Trial Training is especially effective for young children (2 years old) to elementary school children (9 years old) (Bogin, 2008). Research supports that Discrete Trial Training leads to enhancement of communication, social, and behavioral skills (Bogin, 2008). Discrete Trial Training can be used in school, home, or community settings (Bogin, 2008).

Regarding the Autism Treatment Research and specifically the research concerning this particular behavioral program ("Discrete Trials Training and Autism treatment and Early Intensive Behavioral Intervention or Lovaas Institute”), I did not find any study and/or journal article and/or book that investigated the variety of symptoms of the participants (type and intensity), the conceptualization of Autism as a complex phenomenon, or in what ways the research can inform our overall understanding of Autism. I also did not find any comprehensive evaluation of Discrete Trial Training as a complex behavioral intervention.

From all the research reviewed $(\mathrm{N}=23)$ (see appendix $\mathrm{A} 2$, table $\mathrm{A} 2 / 7), 18 \%$ of the research work on Discrete Trial Training was done by means of Single Case Experimental Designs. All of these studies investigated the effectiveness of some relatively specific aspect of Discrete Trial Training such as training effectiveness or speed in responding and concluded with positive results. 
None of the reviewed research work on Discrete Trial Training was done by means of Qualitative Methodology. This clearly indicates a strong positive bias toward an epistemological umbrella rooted in positivism and a strong negative bias toward an epistemological umbrella rooted in constructivism and others. I suggest that the total lack of conceptualization of Autism as a complex phenomenon contributes to incomplete information regarding Autism Treatment Research. The multiple levels of conceptualization and theoretical work (i.e., what it means to be effective for the child with Autism in the context of Autism as a complex phenomenon) also should be especially addressed by tools of qualitative methodology and theoretical instruments.

Five percent of the reviewed research work on Discrete Trial Training was done by means of meta-analysis and $5 \%$ by randomized trial. The meta-analysis results indicated positive changes in intelligence and adaptive behavior and the randomized trial yielded positive changes in intelligence, academic skills, and language but not in adaptive behavior. Fourteen percent of the reviewed articles on Discrete Trial Training were position papers where the authors addressed the predominantly methodological controversy around Discrete Trial Training research. One paper addressed the financial benefit of DTT as opposed to special education. Fourteen percent of the reviewed articles on Discrete Trial Training were conceptualization papers, one of which was a practical guide on how to teach specific language skills. The other author suggested that some of the Discrete Trial Training success might be due to combining other interventions. I consider this conceptualization point important, and as a consequence we clearly have to develop a way to address this issue. The author of the last conceptual paper suggested specific criteria for evidence-based practices. However, these criteria are not grounded in deep theoretical and epistemological accounts. Therefore, these criteria, while clearly developed from positivism, are somewhat arbitrary, ignoring the vast variation of symptoms' 
severity and intensity. I consider these criteria only a preliminary attempt to conceptualize what counts as evidence- based practice. Nine percent of the reviewed articles on Discrete Trial Training were methodological criticisms mainly criticizing or defending the faults in sampling, multiple threats to validity, and data analyses. Twelve percent of the reviewed articles on Discrete Trial Training were book reviews, conference reports, and/or descriptive reports addressing practical issues of Discrete Trial Training implementation. Twenty-three percent of the articles on Discrete Trial Training were group comparisons employing quantitative methodology. All but one of these articles investigated whether or not the Discrete Trial Training is effective.

The studies' results indicated that with Discrete Trial Training, the children improved in IQ, adaptive behavior, language development, and so on. While we do not know what effective means or what kind of meaning these results represent for the children with Autism and their families, the studies concluded that the Discrete Trial Training was successful. One of the studies (Lovaas, 1987) claims that $47 \%$ of the children achieved normal educational and intellectual functioning. However, this particular study was methodologically questioned (Gresham \& MacMillan, 1998) and a randomized trial (Smith et al., 2000) showed not as promising results (statistically significant increase in IQ, academic skills, language development but not a significant difference in adaptive behavior) as Lovaas' original study. Still, the Discrete Trial Training, when quantitatively studied, seems to be a promising intervention. However, without a type of research methodology that will allow us to uncover the "whys" and "hows" (make connections), we will never understand why the intervention is successful and what type of child with what type of specific symptomology will benefit the most. Until we employ rich research 
methodologies and connect the results, we will always have uncertainty. I therefore strongly suggest further research uncovering the actual reasons (factors) contributing to the success.

\section{Subcategory A2: The Koegel Center: Pivotal Response Training}

Method of Systematic Literature Research. For the following literature review I used the descriptor "Pivotal Response Training and Autism treatment and Intensive Early Intervention or Koegel Center" which yielded 128 publications (for which a considerable number of articles were duplicated). Therefore, I reviewed $(\mathrm{N}=39)$ publications to understand what kind of research was completed regarding this specific treatment program for Autism Spectrum Disorder (see appendix 2, table A2/8).

I used the following databases: Education Research Complete, which yielded 21 articles. ERIC yielded 7 publications, Health Science Sources: Consumer Edition yielded 0 articles, Health Science: Nursing/Academic Edition yielded 15 articles, International Pharmacological Abstracts yielded 0 articles, MEDLINE yielded 41 articles, PsycArticles yielded 0 articles and PsycINFO yielded 44 articles. The articles were published in an assortment of different publications between 1979 and 2013.

\section{Overview of the Pivotal Response Training Treatment}

The Pivotal Response Training Treatment is one of the most prominent Applied Behavior Analysis-based treatments. Its main objective is to develop language, communication, and social interaction skills while reducing the amount of self-injurious behavior. The specialty of this treatment is that it focuses on "pivotal areas" rather then some specific target behavior (Koegel \& Egel, 1979). The pivotal areas that are being developed are: motivation, self-management, responsivity to multiple cues, and self-initiated learning interactions (Williams \&Williams, 2011). Another Pivotal Response Training assumption is that it is crucial to train children in 
natural settings (Koegel, et al., 1999). In addition, the family needs to be fully involved and trained via specialized training to reinforce the taught behavior at home (Koegel, et al., 1999).

From all $(\mathrm{N}=39)$ the research done (see appendix 2, table A2/8) regarding "Pivotal Response Training and Autism treatment and Intensive Early Intervention or Koegel Center" from1979-2013, 49\% was done by means of Single Case Experimental Designs. All of these studies investigate the effectiveness of some specific aspect of Pivotal Response Training. For instance, assessment of parents training effectiveness (Coolican et al., 2010), self-management (Koegel et al., 2013), individualized reinforcement (Koegel et al., 2012), embedding social interactions (Koegel et al., 2009). All concluded with positive results.

None of the reviewed research work on The Pivotal Response Training was done by means of rigorous Qualitative Methodology. There was one interview with Lynn Koegel, who addresses general aspects of Autism Spectrum Disorders (Hayes, 2009). Three percent of the research done was completed by means of quantitative methodology, concluding that after employment of Pivotal Response Training, children with Autism significantly decreased their behavioral problems. However, the Autism symptoms decreased only if the participants had an IQ of 50 and above (Smith et al., 2010). Eight percent of the research represents various position papers from describing Pivotal Response Training in Nova Scotia and Canada (Bryson et al., 2007) to discussion about maximizing the effects of the treatment (Koegel et al., 1998). Five percent of the research on Pivotal Response Training took the form of conceptualization papers. Twenty-eight percent of the research done was published in books, book chapters, or book reviews informing parents, teachers, and students on a variety of Pivotal Response Training aspects or procedures. For instance, there is a guide for parents to effectively address multiple questions and issues regarding their children with Autism (Koegel et al., 2009) or a description 
of Pivotal Response Training and the need to keep parents involved closely in their children's treatment (Koegel et al., 2006). Six percent of the research represents dissertations addressing broader aspects of the behavioral treatments, such as the problem of differential responsiveness to treatment (50\% of children with Autism significantly benefit from the treatment but $50 \%$ do not benefit from it) and the need to identify the person's characteristics by creating behavioral profiles that are connected with different outcomes for a specific intervention (Sherer, 2003).

None of these studies investigated the variety of the type, duration, and intensity of the Autism symptoms. Indeed, there were articles that summarized via means of basic literature review the current knowledge about Autism Spectrum Disorder or that addressed some of the challenges. However, none of the studies presented a comprehensive program evaluation of the entire Pivotal Response Training Autism Treatment nor demonstrated and explained how each of the program components produced the changes. A good number of the single case research studies discussed the possible implications of their results for future practice. However, none of the studies connected the results of the single investigation to our overall understanding of Autism as a Complex Phenomenon and in what ways this understanding was enhanced by that study.

\section{Subcategory A3: Strategic Teaching and Reinforcement System: Verbal Behavior}

\section{Method of Systematic Literature Research}

For this treatment of Autism I applied the same system as for other literature review topics. Thus, the literature descriptor was " "Verbal Behavior and Autism treatment and Early Intensive Behavioral Intervention or Strategic Teaching and Reinforcement System” and this yielded 17 publications. However, after I reviewed all the publications, I did not identify any that addressed the Strategic Teaching and Reinforcement System: Verbal Behavior program per se. 
Rather, a variety of Single-Case studies addressing early interventions were the content of this sample. Thus, I changed the literature descriptor to "STARS and Autism Treatment." This yielded 11; however, only three were relevant publications to this program. The rest of them addressed some aspect of Verbal Behavior from B.F. Skinner's teaching.

I used the following databases: Education Research Complete, which yielded 1 article. ERIC yielded 1 publication, Health Science Sources: Consumer Edition yielded 0 articles, Health Science: Nursing/Academic Edition yielded 0 articles, International Pharmacological Abstracts yielded 0 articles, MEDLINE yielded 0 articles, PsycArticles yielded 0 articles and the PsycINFO yielded 10 articles. The articles were published in an assortment of different publications between 1983 and 2013.

\section{Overview of the Strategic Teaching and Reinforcement System: Verbal Behavior}

The Strategic Teaching and Reinforcement System: Verbal Behavior program is based on and derived from B.F. Skinner's behavioral analysis of language (Skinner, 1957). While this program is similar to the Discrete Trial Training, it is predominantly oriented toward language training (Williams \& Williams, 2011). The essence of the treatment approach is that everybody around a child (parents, teachers) requires, prompts, and reinforces a verbal response (Williams \& Williams, 2011).

From all the research done (see appendix 2, table A2/9) regarding "STARS and Autism Treatment", there were three publications. One was a conceptualizing paper that (a) pointed out that the verbal-behavior approach is not empirically well supported (while conceptually sound) and (b) offered the way to collect and publish data for practitioners (Carr \& Firth, 2005). The second was a quantitative methodology dissertation concluding that the behaviorally based Star Program leads to significant improvements in expressive language, receptive language, social 
interaction, and pre-academic skills and significantly fewer stereotypic behaviors (Young, 2007). The third publication was a book addressing/describing the Applied Verbal Behavior method and the need to incorporate development of language early in Autism treatment (Weiss \& Demiri, 2011). The book also described other approaches such as: sign language, video modeling, scripts, and Social Stories. The objective of the book is to provide parents a guide and suggestions to improve communication skills in their children (Weiss \& Demiri, 2011).

In conclusion, it seems that this particular program does not have too much empirical support. There is a possibility that I missed some kind of single-case study, but there is no systematic program evaluation of this program. None of these studies investigated the variety of the type, duration, and intensity of the Autism symptoms. None of the studies presented a comprehensive program evaluation of the entire The Strategic Teaching and Reinforcement System: Verbal Behavior program for Autism Treatment nor demonstrated and explained how each of the program components produced the changes. None of the studies connected the results to our overall understanding of Autism as a complex phenomenon and in what ways this understanding was enhanced by that study.

\section{Category B: Psychological Based Treatments (Relational)}

Williams and Williams (2011) identified the following most prominent psychological Autism treatments. I searched whether or not these treatments for Autism underwent comprehensive program evaluation and found no comprehensive program evaluation of an entire program for these treatments (see appendix 2, table A2/3).

B1: Developmental, Individual Difference: Floortime

B2: The Son-Rise

B4: Gentle Teaching

B5: Holding Therapy 


\section{Category C: Educational Based Treatments (Behavioral)}

Williams and Williams (2011) identified the following most prominent educational Autism treatments. I searched whether or not these treatments for Autism underwent comprehensive program evaluation and found no comprehensive program evaluation of an entire program for these treatments (see appendix 2, table A2/3).

C1: Treatment of Autistic and Related Communication-Handicapped Children

C2: Learning Experiences: An Alternative Program for Preschoolers and Parents

\section{Category D: Neural Based Treatments (Brain)}

Williams and Williams (2011) identified the following most prominent neural Autism treatments. I searched whether or not these treatments for Autism underwent comprehensive program evaluation and found no comprehensive program evaluation of an entire program for these treatments (see appendix 2, Table A2/4).

D1: Sensory Integration Treatment Parents

D2: Vision Therapy Parents

D3: Auditory Integration Parents

D4: Art Therapy Parents

D5: Music Therapy Parents

D6: Facilitated Communication Parents

\section{Category E: Medical Based Treatments (Pharmacological)}

Williams and Williams (2011) identified the following most prominent pharmacological Autism treatments. I searched whether or not these treatments for Autism underwent comprehensive program evaluation and found no comprehensive program evaluation of an entire program for these treatments (see appendix A2, table A2/5).

E1: Amphetamines Treatments

E2: Antipsychotic-Benzodiazepines Treatment 
E3: Antidepressants Treatment

E4: Secretin Treatment

E5: Chelation Treatment

\section{Category F: Biological Based Treatments (Nutritional)}

Williams and Williams (2011) identified the following most prominent biological Autism treatments. I searched whether or not these treatments for Autism underwent comprehensive program evaluation and found no comprehensive program evaluation of an entire program for these treatments (see table A2, table A2/6).

F1: Gluten- Free Treatment - any comprehensive program evaluation

F2: Casein Free Treatment - any comprehensive program evaluation

F3: Vitamin Therapy - any comprehensive program evaluation

In conclusion, it does not take too long of an account of the phenomenon of Autism to see that we are facing a multifaceted problem with many variables. I speculate that the fact that we are handling multiple different variables in a single phenomenon leads us into chaos in Autism Research. I propose that the only way out is creating a system via which we will start to identify and organize these variables. Before we develop some system, we need to conceptualize Autism as a complex phenomenon per se to know what we actually research (complex phenomenon), how we research it (research methods), and what kind of understanding (results/findings) these methods bring and where this kind of understanding leads us. 


\section{Chapter 2: Problem Explanation Conceptualization of Autism as a Complex Phenomenon}

One of the reasons we are not able to identify any unified theories explaining the core and comorbid deficits of Autism is perhaps a consequence of the heterogeneous manifestation of Autism (Levy et al., 2009). It seems that Autism is what could be called an "incongruity phenomenon," but we call it a complex phenomenon. The problem is that we do not know how to study phenomena that are complex; we do not know how to address (describe) and encounter (methodology) complexity in Autism research. Obviously, there are many approaches to studying complexity per se. However, it would be wrong to assume that there is one definition of complexity (Johnson, 2009). Weaver (1991) recognized two types of complexity: (a) the disorganized complexity that he suggested be studied via probability theory and statistics and (b) organized complexity that cannot by studied by statistical tools. Weaver (1991) suggested that organized complexity is nothing more or less than correlated relationships (that are non-random) among parts of the structure/system. In the current literature, I did not find any approaches that conceptualized Autism research vis-à-vis complexity, Autism per se, or the type of evidence for the investigation of complex phenomena that could deepen contemporary understanding of Autism. Thus, in the following treatise, I start to conceptualize complexity regarding Autism (research question 1).

We do identify Autism as a complex phenomenon; however, we do not conceptualize what it means vis-à-vis our Autism research. Therefore, we need to start asking and slowly answering questions such as: how do we recognize and classify complexity? Are there different types of complexities? Why are some phenomena "just" simple? What makes them simple? If they are simple, why do they lack complexity? What are the factors that make complexity 
difficult to study? Is complexity actually something simple but we humans perceive everything we do not understand as complex? Is complexity good or bad? Is complexity purely a manifestation of a constantly changing phenomenon? What are the borders of complexity? Are there any? What research methods have to be developed to be effective when studying complexity? What do we need to do differently? Have we created too many structures around the way we do research and consequently become unable to obtain answers that lie beyond contemporary research structures?

There is much to be done in conceptualization of Autism research. We have not even conceptualized things such as: what counts as progress, what is realistic to expect, what actually is the "Spectrum." We have not conceptualized what kind of research approach we need to use to answer questions such as: what are the reasons behind different manifestations and intensity of symptoms? Why does each child not react in a similar way to treatment? Why do some treatments show significant gains while other treatments produce little or no gains? The fundamental questions for us to ask right now are: What are we missing? What are we not seeing? What kinds of questions are we not asking? What do we need to do, or what do we need to stop doing, or what do we need to do differently to move on to other pieces of the Autism puzzle? And finally yet importantly — what new directions in Autism research do we need to take to advance our current understanding of the condition?

\section{Complex Phenomenon Dimensions}

In the natural sciences such as physics or chemistry, the phenomenon is understood by its inner properties such as chemical, physical, or material characteristics of many kinds.

Researchers have developed sophisticated tools to measure or assess these properties such as: size, weight, color, elasticity, length, intensity, volume, and etc. Some phenomena that natural 
scientists study require less sophisticated research methodology (easily definable and measureable) while others are difficult to study due to more complex dynamics. However, in the social sciences (e.g., human behavior, education, psychological disorders, etc.,), phenomena we study have many dimensions and "behave" differently in different contexts (environments) due to their complex internal dynamics. I suggest that the majority (if not all) of the phenomena studied by social scientists are complex phenomena.

Certainly Autism Spectrum Disorder is a complex phenomenon. In order to significantly move forwards our research efforts, I suggest that we: (a) develop new research methods and/or (b) use the current research tools but in new ways so that we can capture the core of the complexity, hidden to us, in this phenomenon and therefore (c) avoid controversial and contradictory research results and findings. We need to collect richer data and to identify the connections among data so as to reduce the number of contradictory results by means of understanding the dimensions and connections of Autism as a complex phenomenon. This can be accomplished by targeted, purposeful, systematic, and comprehensive program evaluation of Autism treatments.

To encounter complexity intelligently and not in a panic, we must slow down or maybe even stop. We have to get out of the mode of frenetic researching and creating more and more new treatments. Rather we need to conceptualize where we are with Autism research, i.e., what we know about Autism and what we do not know about Autism. What is there that would help us to know, and what is actually important to know, and most importantly, how we will know?

I propose that one of the reasons behind the difficulty in capturing the phenomenon of Autism is that complex phenomena are characterized by many variables and a constant fluidity. Consequently, complex phenomena are very sensitive to any type of changes inside or outside of 
the phenomenon; thus it is extremely difficult to research and consequently to provide stable results. For instance, what are the types of teacher or parent interactions and the types of environments that will influence (in some direction) the feelings and the behavior of a person with Autism? Also, what are the biological and neurological components of a person with Autism that will influence their interactions with other people?

I suggest that each complex phenomenon has its own dynamics. These dynamics have certain properties. The number and the types of what I term Complex Phenomenon Dimensions ${ }^{5}$ encapsulating the phenomenon define this property. By a dimension I mean something like a variable. However, contemporary research predominantly understands variable as something that has a one-dimensional fixed quantity and therefore is relatively easy to measure. If we were to pursue research regarding Autism more like searching for dimensions, then we would open up to the likely possibility that one dimension has multiple quantitative and qualitative characteristics which indeed are challenging to measure and will require more than one measurement tool. Consequently, we would be open to the larger spectrum of variation. Such variation we can easily observe in Autism symptoms, which are manifested in each individual differently; they have different intensity or duration and the symptom types vary from person to person.

Each dimension (multifaceted aspect of the phenomenon) has its own degree of internal variation and level of intensity. The dimensions are mutually interconnected, creating a special type of interactive system. This interactive system is the internal (to contemporary measures hidden) dynamics of complex phenomena. I propose that we have to study the internal dynamics that I define as a multi-relational (connections) and multi-dimensional (multiple quantitative and qualitative characteristics) interactive process leading to variable representations of Autism

\footnotetext{
${ }^{5}$ Complex Phenomenon Dimension: A variable with multiple qualitative and quantitative characteristics.
} 
manifestations, which I propose is the ultimate core of the complexity. Consequently, we need to identify and employ the types of methods, procedures, and measurements that will allow us to uncover these dynamics; consequently, we will reach a profound understanding of Autism.

This Autism interactive process we can call Autism Dynamics ${ }^{6}$. Consequently, Autism cannot be a static phenomenon but rather can be easily perceived as a moving target or gelatin with no clear set of boundaries, manifestations, definitions, prevalence, strong biological heterogeneity and etc. Therefore, we have a hard time understanding the variability of symptoms, and thus cannot explain the phenomenon of differential responsiveness [50\% of children with Autism benefit from the treatment but 50\% do not (Sherer, 2003)].). Autism cannot be defined by one definition or one single explanation. Consequently, Autism cannot be explored by use of one single research method or one single type of research results.

For instance, Autism treatment research predominantly studying the impact of Applied Behavior Analysis- based treatments almost exclusively employs Single-Case Experimental Designs as their research method. Now, this particular research tool is superior for evaluating the direct impact of a specific part of a single intervention on a target behavior for a single individual or a single group of individuals. Single-Case Experimental Designs are especially effective when researchers need to use frequent measurements of a single intervention outcome to see if and when the outcome changed. Now, while this research tool is dynamic, as it develops in a direct response to actual data patterns rather than an a priori set plan for data analyses, it cannot fully uncover the dynamics of Autism. Simply, a single method cannot lead to understanding of the multi-relational (connections) and multi-dimensional (multiple quantitative and qualitative

\footnotetext{
${ }^{6}$ Autism Dynamics: a multi-relational (connections) and multi-dimensional (multiple quantitative and qualitative characteristics) interactive process leading to variable representation of Autism manifestations
} 
characteristics) Autism interactive process. Consequently, the use of only Single-Case Experimental Designs (or any other exclusive research method) cannot lead to a profound understating of Autism as a Complex Phenomenon. Rather, the employment of multiple research methods and the development of new methods will eventually lead to uncovering the dynamics of Autism. To succinctly illustrate the problem of the use of one single method originated in one single epistemological umbrella while researching complex phenomena characterized by enormous variability, it is like cooking a complicated meal with only a wooden spoon.

\section{Culture of Evidence versus Culture of Exploration}

Even in specialized and focused Autism treatment research, we need to develop a bigger picture as opposed to a one-dimensional and linear snapshot of a treatment's effectiveness. There are many voices stressing the need to evaluate treatments for Autism (Levy, et al., 2009). We have a strong need for demonstration of the evidence of the efficacy of behavioral interventions (Reichow et al., 2008). We seem to be able to make a conclusion that treatments based on behavioral science are empirically validated as effective (Schreibman, 2008). On the other hand, we seem to be able make a conclusion that some other treatments such as Facilitated Communication or Option Therapy are ineffective (Schreibman, 2008). I do concur with Schreibman and others that it is important to know what treatments are effective and what treatments are ineffective. However, I do not think that "only" this will bring us any closer to a profound understanding of Autism as a phenomenon.

I suggest, given the omnipresent variability in the phenomenon of Autism, that we conceptualize first what actually counts as evidence in Autism treatment research. Before we decide whether such and such treatment is effective or ineffective, we need to determine whether 
we can use the same way of building evidence and the same type of evidence, but across different types of problems - particularly when studying complex phenomena.

I propose that first we need to ask, what is evidence? I view evidence as [something] or data collection that is developed according to particular rules or epistemology to make an objective judgment and/or decision. This [something] or data collection we try to collect and construct in a way that will lead to a clear indication of some kind of behavioral or other type of pattern. In the context of Autism Research, evidence is assumed to be a result of rigorous, preferably quantitative, empirical studies. Specifically, according to what research methods we used, we are talking about strong or weak evidence. Maurice, Green, and Luce (1996) define evidence to determine whether or not an Autism treatment is effective as:

$>$ Results have to be measurable

$>$ The measures have to be objectively observable by direct observation

$>$ Conditions have to be systematically manipulated

$>$ Results have to be replicated

It is clear that epistemological beliefs (in this case positivism) determine what research methodologies will be used to gather the "right" type of evidence. Consequently, we are depriving our research process of a richer picture or unexpected evidence because we do not consider any other types of evidence. Consequently, our understanding (research results) has only linear and one-dimensional characters moving from strong to weaker to weak evidence. However, we are handling an extremely variable phenomenon that has a multi-relational (connections) and multi-dimensional (multiple quantitative and qualitative characteristics) interactive process. What we so far consider as evidence needs to move from one-dimensional linearity to a multifactorial understanding of the context. 
I suggest that we develop a larger picture regarding treatments' success by identifying and describing particular reasons explaining why some treatments are effective; the particular reasons why some treatments are not effective; and the reasons that some treatments are effective for some individuals but not for others. These reasons we can conceptualize as factors and the factors as single tiny pieces of a large and colorful puzzle depicting the condition of Autism. To gain this kind of insight into Autism treatment vis-à-vis a person with Autism and his/her environment, not only do we need to measure changes in specific behavior, when they happened, if they happened, and under what conditions they happened, but also to gain understanding of how each part of the treatment contributes, how the parts function as a whole, what are the connections between and among the treatment's parts, and how they impact the person with Autism and his or her immediate environment.

I suggest that our way (method) of investigating complexity determines the speed, depth, and precision of our research. While many of us can argue that Autism research is producing many studies, others may argue the results are not comprehensive and that we have to admit that we have a lot to learn. We are somewhat successful in describing Autism symptoms predominantly via behavioral description. However, we have not discussed how we would learn about the internal interactive process (Autism Dynamics). And we have not even started the discussion about the relationship between what we consider as evidence and what we learn as a consequence, let alone discussed the need to broaden our epistemological grounding in Autism research. The prison of one epistemological umbrella will not permit development of research methods or redesigning current ones that will lead to different types of learning in order to understand the different dimensions of Autism. 
To significantly advance Autism research, we need to ask the fundamental question: how to do we successfully study Autism? From the conceptualization of Autism as a Complex Phenomenon, it follows that we need to employ research methods that will have the power to uncover the interactive process of Autism. Therefore, we need to employ research methods that will allow us to identify:

a) The number of dimensions of Autism as a Complex Phenomenon

b) The dimensions' degree of variation

c) The dimensions' levels of intensity

d) The connections and the types of connections between and among dimensions

When we (researchers) encounter a complex phenomenon such as Autism, usually we are not aware of its interactive process. We do not know how many dimensions make up the complex phenomenon under our study. We do not know how many and what type of relations (connections) there are among dimensions; thus we do not know how the interactive process works. While we study complex phenomenon such as Autism, we tend to employ rigorous research methods but can arrive at contradictory results - we arrive at what I call A Complex Phenomenon Paradox ${ }^{7}$. I speculate that this Complex Phenomenon Paradox arises when:

(a) We use the same or similar research methods to explain different dimensions of Autism as a complex phenomenon.

\footnotetext{
${ }^{7}$ Complex Phenomenon Paradox: While using rigorous research methods and arrive at contradictory results
} 
(b) We try to explain the complete Autism puzzle with only one single puzzle piece. That is exactly why it is critical to identify how many dimensions a complex phenomenon has and what particular parts we are studying.

To effectively investigate complexity, we will have to adjust our way of thinking, viewing, and doing research. One possible way to start can be to free our research from what I term as Simpleness ${ }^{8}$. Simpleness, I propose is [something] simple because this [something] has only one dimension. If this uncompounded [something] has only one dimension, then once we uncover and describe that dimension, we understand it and do not need to worry about the internal dynamics. Simpleness (one-dimensionality) in Autism treatment research can be exemplified by our predominant tendency to only research (evaluate) if this or that treatment for Autism is effective (one-dimension) or ineffective (one-dimension). While such a research objective is perfectly logical, and essential for the children with Autism and their families, it is not a productive way to handle complexity.

If we take into an account my conceptualization of Autism according to which Autism is a multi-relational (connections) and multi-dimensional (multiple quantitative and qualitative characteristics) interactive process leading to variable representation of Autism's manifestation, then a one-dimensional result (such and such treatment is effective or ineffective) will not bring us any closer to a profound understanding of Autism as a Complex Phenomenon. In other words, the research results determining that treatment $\mathrm{A}$ is effective and treatment $\mathrm{B}$ is ineffective will lead us to the status of the current Autism Treatment Research — it is trapped deep in simpleness. The trap is that things in Autism are simply not that simple. They are neither

\footnotetext{
${ }^{8}$ Simpleness: Something that has only one dimension.
} 
white (treatment A is effective) nor black (treatment B is ineffective). Is treatment A or B only partially effective or ineffective? The problem with this type of research (simple determination of efficacy of treatments) is that it reduces a complex phenomenon to simpleness (something that has one dimension), and that is exactly what Autism is not. Autism symptoms do not have one dimension; Autism treatments do not have one dimension. Correspondingly, we need to open up to complexity, thus multidimensional thinking, and to start to approach Autism research accordingly. In short, any type of phenomenon characterized and manifested by complexity cannot be understood from a dualistic (either ... or) perspective.

Furthermore, there are many possible variations of what we call effective. I strongly suggest that before we go ahead and identify which treatment is effective and which is ineffective, we conceptualize what it means to have "an effective treatment for Autism." In my understanding [something] is effective if it achieves the proposed effect. Parents and practitioners should especially contribute to our understanding of what it means to have an effective treatment for Autism. Consequently, qualitative researchers via use of qualitative methodology should also participate on the development of what I term Categorization of Effectiveness ${ }^{9}$ for Autism Treatments.

Thus, before we identify what is an effective treatment, we need to know what exactly is the proposed effect. Is this proposed effect complete eradication of all (or some) of the Autism symptoms? Is it mitigation of all (or some) of the Autism symptoms? If it is mitigation of symptoms, what is the degree of mitigation? If it is mitigation or elimination of some of the symptoms, what are the specific symptoms? What are the characteristics of the individuals for whom such and such treatment works? What are the characteristics of the individuals for whom

\footnotetext{
${ }^{9}$ Categorization of Effectiveness: Different degree of Autism Treatment impact.
} 
such and such treatment does not work? In what ways do these characteristics differ? What do these characteristics have in common? How do the symptoms and their intensity differ, and in what ways do these differences impact the treatment outcomes? We also need to learn to value information that carries a so-called negative message (treatment is ineffective). This is for us as valuable information as the opposite (treatment is effective) because it moves us closer to a profound understanding of Autism as a Complex Phenomenon and its nature.

\section{Levels of Complexity}

I suggest that we recognize that phenomena are complex and that this means the way we approach research is not sufficient. Therefore, I propose that we need to think about different levels of complexity as well. Complexity is a multi-relational (connections) and multidimensional (aspects) interactive system (dynamics). This definition implies that once we uncover the dimensions and their degree of internal variation, level of intensity, and type of connections, we can come to fully understand any type of complex phenomena such as Cancer. This definition implies that the levels of complexity are the same across different types of complex phenomena. However, such a type of thinking would lead to a reduction of complexity. Therefore, I propose that my own definition of complexity [multi-relational (connections) and the multi-dimensional (aspects) interactive system] is limited because it does not take into account the possible variation of complexity.

I suggest that the dimensions may or may not involve other sub-dimensions that are creating the sub-interactive systems within the main internal interactive system of the complex phenomenon. Therefore, we can develop levels of complexity such as complexity Type A, complexity Type B, complexity Type C or complexity Type AB, Type ABC, etc. There are infinite possibilities for complexity transmutations that are results of change. Any type of change 
outside or inside complex phenomena may lead to phenomenon transmutation. Hence, I think that to come up with Levels of Complexity for Autism is a good idea, however premature it may be at this moment and given the level of our understanding.

\section{Primary Autism Complexity Division}

Laura Schreibman (2008) argues that the fact that we do not have a "cure" for Autism results in a situation where there are all kinds of treatments; but little scientific evidence to base them on. It seem that we have two options here: (a) wait for the research results that would uncover the etiology of Autism or (b) go ahead and try to develop treatments that at least we know will not hurt children with Autism.

It seems that we are trying to hit the target (uncovering optimal treatment) while we have no idea where to find the target (cause). I do not think that we need to position our research in a dual situation where we have only two options: either to wait for the uncovering of Autism's etiology or to develop somewhat blind treatments. Besides, this reasoning (waiting for the cause or developing haphazard treatments) would lead us to simpleness (something that has one dimension). However, we need to start to approach this research in a way that effectively addresses the complexity of Autism. Therefore, we need to avoid dualistic thinking while approaching complex research problems.

I suggest the following method (Primary Autism as a Complex Phenomenon Division) for our current situation in Autism research. I suggest that the Autism Complexity Division method will prevent dualistic thinking and also break down complexity into manageable and focused research.

In Autism Research, let's assume (for now) that there are four main complexity divisions: (see figure 2.2.) 
Autism Etiology Complexity Division

Autism Symptoms Complexity Division

$>$ Autism Treatments Complexity Division

$>$ Autism Treatments Outcome Complexity Division

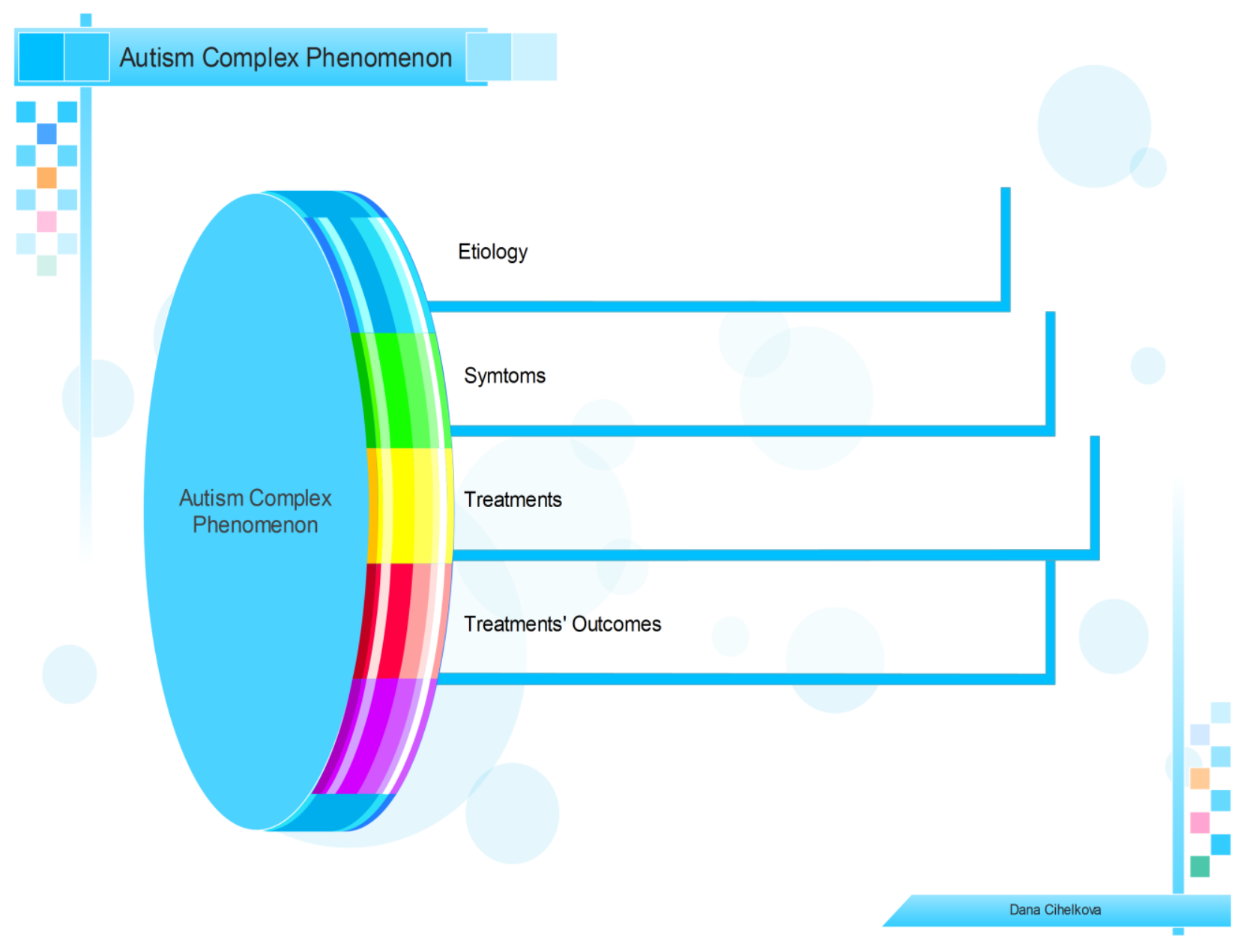

Figure 2.1. Autism as a Complex Phenomenon Division. This figure illustrates the four main complexity divisions of Autism as a Complex Phenomenon

Primary Autism Complex Phenomenon Division (see the figure 2.1. below) is a basic method that will allow us to handle the complexity of Autism by narrowing and focusing our research efforts to a particular space. 


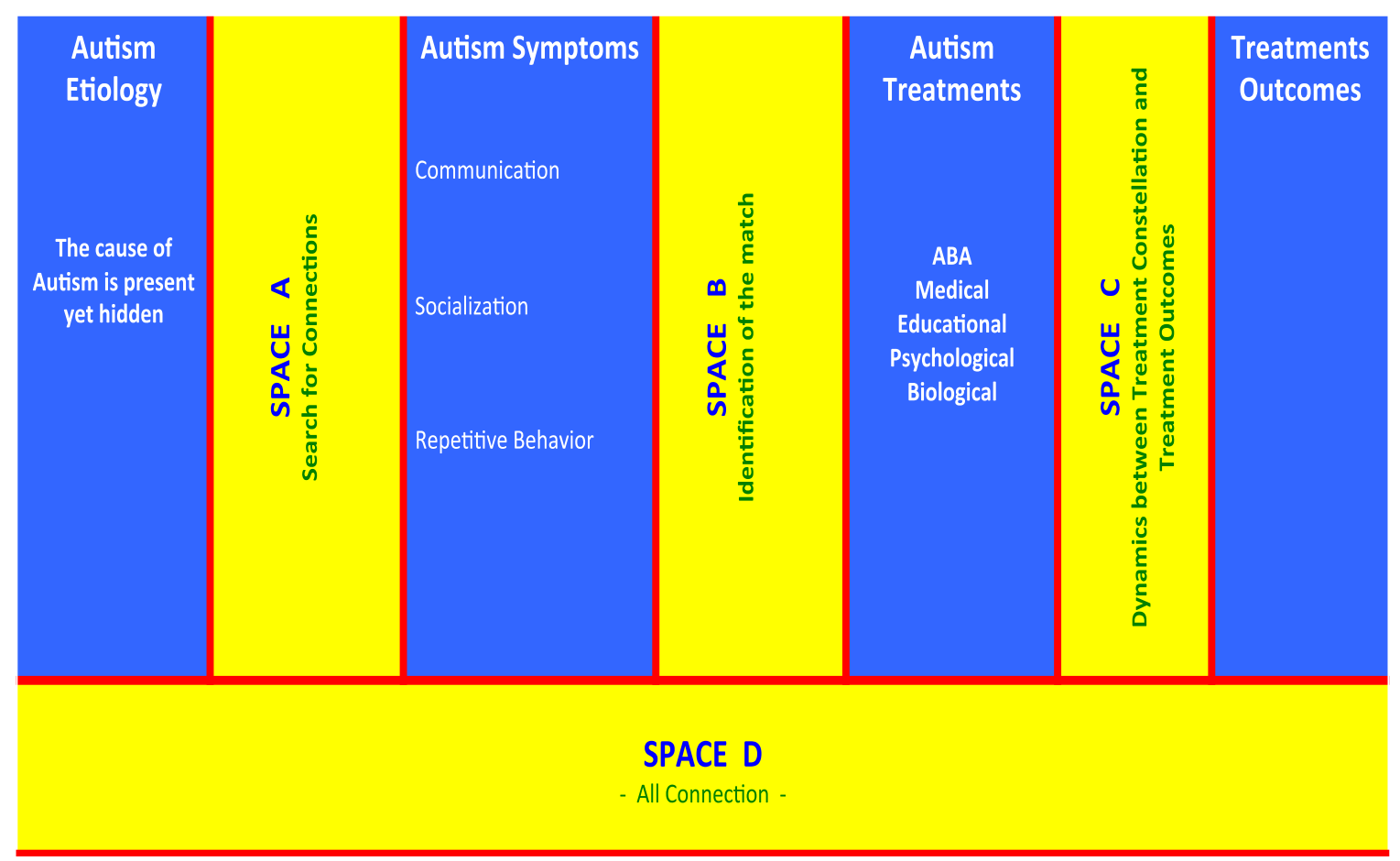

Figure 2.2. Primary Autism Complexity Division. This figure illustrates a method for break down the large complexity block into a manageable and focused research

This method ${ }^{10}$ has one basic assumption: the larger the research space we are investigating, at one time, while not knowing the dimensions and connections of the interactive process, the less satisfactory and the more contradictory, and confusing the results/understanding we produce. In other words, we are everywhere and nowhere with Autism Research. If, however, we narrow the research space (complexity) down so that it is manageable and then extend that space once we understand the inner dynamics of that specific space, and then extend it more, and so on we will gradually gain understanding of the complete complex phenomenon.

\footnotetext{
${ }^{10}$ Primary Autism Complexity Division: a method for break down the large complexity block into a manageable and focused research
} 
If we narrow down the research space, we will (a) focus our research, (b) handle a manageable sized space, and most importantly (c) be able to gradually understand the connections (and types of connections) between and among dimensions of Autism as a complex phenomenon. Let's be specific.

Each complexity division (etiology, symptoms, treatments, and treatments outcome) has an unknown number of dimensions and dimensions types. Between each complexity division is a certain space with, to us, an unknown number of connections and connections types. To handle the unknown properties, we can break down the enormous Autism research space into four different yet interconnected Autism Research Sub-spaces of [Space A (etiology $\leftrightarrow$ symptoms), Space B (symptoms $\leftrightarrow$ treatments), Space C (treatments $\leftrightarrow$ treatment outcomes), and Space D (treatments outcomes $\leftrightarrow$ etiology). This will allow us to uncover important connections. Space A (Etiology $\leftrightarrow$ Symptoms) (See figure 2.1.)

In Space A, we can investigate the dimensions and the connections of Autism Etiology Complexity Division and Autism Symptoms Complexity Division, with the key objective to develop a theory of Autism causation. We know that [something] is causing Autism. However, we do not know what that [something] is. That leads us into today's situation where we are developing blind, less or more successful, treatments and have no research toward Autism prevention. Nevertheless, we do know the symptoms of Autism. There are three main symptoms: disruptions in communication, impaired social interaction, and repetitive behavior. I term these symptoms the Primary Autism Symptoms ${ }^{11}$. However, the intricacy of Autism is manifested by the vast variability of types and different levels of intensity of the Primary Autism

\footnotetext{
${ }^{11}$ Primary Autism Symptoms: Disruptions in communication, social interaction, and repetitive behavior.
} 
Symptoms in each individual, creating what I term Individual Autism Symptoms ${ }^{12}$. Our research task in space A is to uncover the unknown yet present cause or causes of Autism. Therefore, we need to employ research methods that will allow us to uncover the dynamics of the indirect expression of Autism's cause.

Space B (Symptoms $\leftrightarrow$ Treatments) (See figure 2.1.)

In Space B, we can investigate the dimensions and the connections of Autism Symptoms Complexity Division and Autism Treatment Division with the key objective of identifying the matching treatment for such and such manifestation of Primary and Individual Autism symptoms in each child. The current situation in Autism treatment research is that we have a variety of treatments, from psychological to medical to biological to behavioral to educational, but we lack comprehensive and purposeful evaluations (see appendix, Table A2/2) of these treatments to be able to answer how they impact the Individuals with Autism.

Consequently, we cannot learn about the dynamics between such and such symptoms combination and such and such treatment constellation. Ultimately, we cannot identify the optimal (currently available) treatment for such and such manifestation of Primary and Individual Autism symptoms in each child. In addition, if we reach a complete understanding of this space (Space B), we can make, so far to us, hidden connections and inform the other researchers working on a different Autism Complexity Division. I developed a research tool, (the Dynamic Evaluation Model), that will allow us to uncover the optimal treatment match according to the Primary and Individual Autism Symptoms (See chapter 3: The Dynamic Evaluation Model)

\footnotetext{
${ }^{12}$ Individual Autism Symptoms: Different types and level of intensity of primary Autism symptoms.
} 
Space C (Treatments $\leftrightarrow$ Treatment Outcomes) (See figure 2.1.)

This space is similar to Space B, but in this case we will gain understating via The Dynamic Evaluation Model into the dynamics between such and such a treatment constellation and particular treatment outcomes. The knowledge from this space can lead to development of a three-dimensional matrix mapping (relating) the specific treatments constellations, Autism symptoms constellations, and treatment outcomes.

Space D (Treatments Outcomes $\leftrightarrow$ Etiology) (See figure 2.1.)

To investigate Space D is perhaps the least fruitful way to progress in Autism Research. It is simply a very large and thus hardly manageable research space. We need to uncover the necessary connections from the sub-spaces $(\mathrm{A}-\mathrm{C})$ first to be able to see the interactions between etiology and treatment. For instance, a research study that proposed that vaccination is behind Autism was trying to capture all the spaces at one time. Wakefield (1998) concluded that vaccination causes Autism. Therefore, the obvious treatment was not to provide vaccinations for children. The researcher crossed all the spaces, however, without knowledge of the sub-spaces dimensions number and types and connections number and types. In the study of complex phenomena, such a simple (one-dimensional) solution can hardly be the answer (treatment) for the problem (Autism) the inner essence of which is complexity (a multi-relational (connections) and the multi-factorial (aspects) interactive system.

In conclusion, research on Autism is enormous and so far has been broadly divided into two main areas: (a) basic research that searches for the cause and expression of Autism and (b) applied research that searches for effective treatment via identification and evaluation of current treatments (Romanczyk, 1999). Given the complexity of Autism, I suggest that we differentiate 
among Autism Etiology Research ${ }^{13}$, Autism Treatment Research ${ }^{14}$, Autism Epidemiology

Research ${ }^{15}$, Autism Family Research ${ }^{16}$ and Autism Prevention Research ${ }^{17}$ so that we can:

a. Systematize our effort by breaking down the huge Autism research efforts

b. Pass research findings from one Autism Complexity Division to others without loss of valuable data

c. Increase Autism research's efficiency

d. Increase the speed of our research

My main dissertation contribution is development of an evaluation model to evaluate Autism treatments and investigate Spaces B and C in Autism Complexity Division. The Dynamic Evaluation Model has the capacity to significantly advance knowledge in the area of Autism treatment research by creating a three-dimensional matrix of intervention components, child symptoms, and behavior outcomes. Therefore, stakeholders will be able to make informed decisions about what type of treatment can best address various manifestations of Autism spectrum symptomology.

\footnotetext{
${ }^{13}$ Autism Etiology Research: Searches for a cause or causes of Autism

${ }^{14}$ Autism Treatment Research: Searches for optimal treatments for variety of symptoms manifestation

15 Autism Epidemiology Research: Searches for reliable system of count and true pattern of Autism prevalence

${ }^{16}$ Autism Family Research: Uncover the variety of impacts of the Autism on family units

${ }^{17}$ Autism Prevention Research: Searches how to prevent Autism
} 


\section{Chapter 3: Problem Solution The Dynamic Evaluation Model}

Government and accreditation programs highlight the necessity to increase and assess students' academic outcomes, intellectual development and overall educational achievements (Brittingham et al., 2008). The same situation exists in the behavioral domain; where there is a strong need to demonstrate the efficacy of behavioral interventions (Reichow et al., 2008).

Kasari (2002) suggests that there are only limited studies for behavioral treatments for Autism. Stakeholders have the need to identify behavioral models that demonstrate positive outcomes for individuals with Autism Spectrum Disorder (Odom et al., 2010). However, the comprehensive behavioral interventions/models lack systematic evaluation (Odom et al., 2010).

Parents criticize the lack of practical information and research regarding effective treatment for their children (Granpeesheh, Tarbox, \& Dixon, 2009). There are many treatments for Autism that are eagerly supported and researched by the developers of these treatments, but the vast majority was not subjected to rigorous research evaluations (Granpeesheh et al., 2009). This situation leads parents and other stakeholders to a position where they are not able to identify the most productive or suitable treatment for children with Autism or other severe developmental disabilities. "Although it would be wonderful if parents and professionals had an evidence-based algorithm to clearly identify which treatment method is best for each child on the autism spectrum, no such algorithm exists" (Tchaconas \& Adesman, p.137, 2013). The question therefore is what is the best methodological tool to accelerate the assembly of nationwide evidence-based Autism treatments in order to identify the optimal treatments for the variety of different children with Autism (research question 2). Like Tchaconas and Adesman, I also did not find any evaluation model that would have this capacity or an evaluation model that could be 
used to develop this type of model — to identify which treatment is best for each child on the autism spectrum. For this reason, I developed an original Dynamic Evaluation Model for Evaluation of Complex Behavioral Programs and other treatments for Autism. Furthermore, the advantage of this model is that it can be applied, with modification of the measurement types, to any other type of evaluation of complex psychological, educational or medical phenomena.

I suggest that one of the (many) reasons that complex behavioral interventions are not comprehensively evaluated or not evaluated at all is that behavioral interventions are not onedimensional. Rather, behavioral interventions generate an open system. By open system I mean that behavioral interventions are more or less complex combinations of parts that aim to produce a certain type of behavior (behavioral repertoire). Therefore, behavioral interventions are made up of many parts designed to develop a multifaceted set of behaviors. For instance, The Competent Learner Model aims to develop seven specific behavioral repertoires (Warash et al., 2008). While the Competent Learner Model was to some degree conceptualized, the Competent Learner Model was never conceptualized in terms of how the development of the seven repertoires contributes to the treatment of Autism. Thus, stakeholders do not know how each part and the combinations of the parts contribute to the changes in the constellations of symptoms that lead to a diagnosis of Autism for the children they serve or parent.

The majority (if not all) complex behavioral interventions are based on Applied Behavioral Analysis (Granpeesheh, 2009). Because behavioral interventions represent open systems, their success depends on how well the interventions interact with the environment. This requires productive connections with the environments of families, schools, communities, and society; otherwise such interventions may become Autistic in their nature as well. Therefore, the 
degree of the productivity of these interactions (connections/relations) has to be assessed and become part of the comprehensive evaluation.

Complex behavioral interventions cannot be fully evaluated by exclusively using Single Case Research Designs because these types of designs are not well suited for the evaluation of entire programs. While the results from the Single Case Research Designs are valid, rigorous, and show functional relations between particular treatment components and changes in target behavior, these types of results cannot uncover the dynamics of the entire behavioral intervention system. Rather, I suggest that a variety of research methods, data, and data analyses need to be employed not only to measure changes in behavior but also to gain understanding of how each part contributes, how they function as a whole, what the connections are among parts, and how they impact the immediate environmental context. Fundamentally, what we need to do in order to truly understand the impact of behavioral interventions and other treatments for Autism is to evaluate the dynamics of the behavioral interventions. This tactic, evaluation of the intervention dynamics, will lead to comprehensive evaluation and understanding of the consequences of complex behavioral interventions.

\section{Logic Models vis-à-vis Evaluation of Autism Treatments}

In the contemporary literature I did not find any model for comprehensive evaluation of Autism treatments. Perhaps the most common instrument for evaluation of large programs (in general) is the Logic Model, which is used predominantly by governmental organizations. The main purpose of the Logic Model is to provide a plan for evaluation of a program and to develop evaluation questions (Isaacs, Clark, Correia, \& Flannery, 2009). The advantage of the Logic Model is that it helps to uncover the course of the necessary procedures [actions] and the short and long term outcomes of implementing those procedures (Isaacs et al., 2009). The Logic 
Model is essentially a vehicle to develop and deliver a visual representation of the way in which a program or intervention will produce its intended outcomes.

I argue, however, that the Logic Model is not sufficient for evaluation of complex behavioral programs or other interventions for Autism. The vast majority of the Logic Models operate on a linear sequence of: inputs $\rightarrow$ activities $\rightarrow$ outputs $\rightarrow$ outcomes. There are many types of Logic Models, which include the elements of Inputs, Activities, Target Groups, Outcomes, and Outputs (Cooksy, Gill, \& Kelly, 2001).

While there is a variety of Logic Models, they all operate on a linear and a priori predefined sequence of evaluation of outcomes. The significant benefit of the Logic Model is that it helps evaluators to rapidly orient in the path of the evaluation and identify the particular measurements for the anticipated outcomes by means of a visual representation. Nevertheless, I argue that the weakness of a Logic Model is that it views an evaluated entity (program, intervention) as a closed system. For the evaluation of complex behavioral and other interventions for Autism, this closed system assessment will not allow learning beyond the predefined evaluation path of the Logic Model.

There is no single system that is truly isolated, that does not interact with (impact or be influenced by) the external environment. We, however, think of many models as closed systems that help us to understand reality, such as mathematical or statistical models. The problem is not in using these isolated models but in our tendency not to conceptualize these models as only better or worse approximations of reality. To avoid the trap of putting too much weight on statistical and/or mathematical models, we need some supplementary information (data). Therefore, I suggest developing an evaluation model for Autism treatments that will bring attention not only to outcomes but also to context. However, no such type of model exists. 
For this reason, I developed the Dynamic Evaluation Model for evaluation of complex behavioral programs or other treatments for Autism. The dynamic properties of the model will allow evaluators and/or practitioners to engage in iterative and open system evaluation.

Moreover, we can use this tool to advance contemporary knowledge about Autism by developing Autism Symptomology Dynamics, which may explain the phenomenon of differential responsiveness [e.g., $50 \%$ of children with Autism benefit from the treatment but $50 \%$ do not, Sherer (2003)].

\section{Theoretical Assumptions of the Dynamic Evaluation Model}

The Dynamic Evaluation Model's underlying assumption is that complex behavioral interventions are not one-dimensional but rather complex systems. Therefore, complex behavioral interventions for Autism need to be evaluated as a system. The model's assumptions are that:

$>$ Each behavioral intervention has certain types of dynamics that are designed to produce certain types of behavioral outcomes and that these outcomes can be measured.

$>$ The intervention dynamics influence and are influenced by the environmental context of the intervention and that this context can be measured.

$>$ Each complex behavioral intervention has certain structure and therefore the intervention can be described and explained.

$>$ Complex behavioral interventions attempt to change behavior and the degree of these changes can be measured.

$>$ Behavioral interventions form open systems that can be studied.

$>$ These systems are comprised of numbers of parts that are mutually interconnected to achieve a proposed effect that can be measured.

$>$ The Dynamic Evaluation Model cannot draw a certain conclusion about causality between the parts or sum of the parts of the intervention and the outcomes. 
$>$ The Dynamic Evaluation Model can identify, describe, and correlate the intervention parts with the outcomes.

$>$ The dimensions of the Dynamic Evaluation Model define the scope of the evaluation.

$>$ To uncover the intervention dynamics, intervention impacts, and the relations between interventions and environment requires multiple perspectives, data collection, and analyses.

The Dynamic Evaluation Model was developed as a reaction to a critical need to comprehensively evaluate complex behavioral programs for the treatment of Autism. The model's power is in its relative simplicity that allows for the design of evaluations that are doable because the model connects the currently available and commonly used standardized measurements with measurements of contextual understanding to integrate results from each evaluation dimension. This method will lead to multiple levels of evaluation outcomes and to an elegant synergy between practice and theory.

The Dynamic Evaluation Model represents a certain type of theoretical evaluation construct to produce comprehensive evaluations of behavioral and other treatments for Autism. The existing interventions produce an enormous amount of rich data but do not have ways to accomplish comprehensive evaluations from the data. The Dynamic Evaluation Model offers practitioners a guide to accomplish systematic and comprehensive evaluation. Essentially, it is a tool of program evaluation that will inform general research on Autism and research regarding treatments for Autism. The practitioners will learn how the treatments work, what needs to be further developed or enhanced, what does not work, and with whom the treatments work the best. Identifying the distribution of Autism symptoms and uncovering the dynamics among the symptoms will inform research in the areas of Autism etiology, and pharmacology will be informed about many aspects of Autism symptoms and specific treatments responsiveness. 


\section{The Dynamic Evaluation Model}

The Dynamic Evaluation Model is a five-dimensional evaluation method to collect a large specific data set from Autism programs. The model has five dimensions: (1) Conceptualization, (2) Diagnostics, (3) Environment, (4) Outcomes, and (5) Understanding (see Table $\mathrm{X}$ in appendix for a graphic representation). Each phase employs a different research method, collects different types of data, and employs different data analyses. Therefore, each evaluation phase (dimension) will lead to different types of results, allowing for more complete understanding. After employment of the model, we will be able not only to determine if such and such treatment is effective, but also to learn about the Autism condition per se (See specific model outcomes in Dimension 5:Understading).

The general objective for dimension one is to produce evidence-based knowledge about how complex behavioral interventions work. The general objective for dimension two is to identify who benefits the most from a particular complex intervention. The general objective for dimension three is to produce evidence that identifies specific ways in which complex behavioral interventions impact the immediate environment and vice versa. The general objective for dimension four is to quantify changes in behavior by assessing the impact of the complex behavioral intervention. And finally, the general objective for dimension five is to integrate the data and provide contextual understanding of the effectiveness of complex behavioral interventions. 


\section{Dimension 1: Conceptualization}

The purpose of this evaluation dimension is to develop the Structural Framework ${ }^{18}$ of the complex behavioral intervention. The purpose of the structural framework of the intervention is to identify and describe the specific elements and their relationships. The purpose of the identification and description of the interventions elements and their relationships is to identify the functional connections among intervention parts and to measure the degree of these functional relations. The purpose of identification and measurement of degree of functional connections is to uncover how and to what degree each intervention part and the combination of the intervention parts contribute to changes in the behavior of the child with Autism. To develop the structural framework of the intervention, the developers will answer the follow questions:

Name of the intervention

Theoretical grounding of the intervention (e.g., Applied Behavioral Analysis)

Identified parts of the intervention

$>$ Number of parts in the intervention

$>$ Objective for each part

$>$ Functional relations among parts

Strategies to achieve the proposed effect

Intensity of the intervention (time)

$>$ Epistemological assumptions

Short-term outcomes

$>$ Intermediate outcomes

\footnotetext{
${ }^{18}$ Structural Framework of the Treatment: Identification and description of intervention parts and relationship
} among parts. 


\section{Ultimate outcomes}

\section{Dimension 2: Diagnostics}

The evaluation objective of this dimension is to develop a table of Autism symptoms' intensity and frequency, thus their distribution. The purpose for understanding the distribution of Autism symptoms is to achieve an understanding of the dynamics of Autism symptomology by uncovering the relationships among symptoms. The purpose of gaining an understanding of Autism symptomology dynamics is to identify what treatment will work best for what symptom characteristics.

Furthermore, the ultimate complexity in evaluating treatments for Autism is that individuals with Autism are diagnosed according to some degree of presence or absence of multiple Autistic behavior patterns described by DSM-V. Different degrees of symptom intensity and different types of symptoms create enormous variability among individuals with Autism. Due to this variability, it is impossible to achieve a fair comparison of Autism treatments. Additionally, due to the phenomenon of differential responsiveness [e.g., $50 \%$ of children with Autism benefit from the treatment but $50 \%$ do not, Sherer (2003)], it would be premature to make a conclusion that treatment $\mathrm{A}$ is better than treatment $\mathrm{B}$ given the possibility that such and such a child with such and such Autism symptoms may benefit from this treatment but not that treatment. Indeed, we can achieve a fair comparison of treatments for Autism by using the same measurement methods to measure the same changes in Autistic behavior. However, this assumes that each child responds to the treatment in the same way. The phenomenon of differential responsiveness suggests that a simple comparison of Autism treatments can lead to an oversimplification of this complex problem and therefore to misleading conclusions. 
Accordingly, rather than compare treatment to treatment we need to understand the differences in the treatments and how these differences impact the behavior of individuals with Autism.

Therefore, the aim of this phase is to collect data (e.g., detailed demographics and frequency, intensity, and functionality of behaviors) from each complex intervention program and assess what patterns in these data are associated with the outcomes produced by the programs. This way we can avoid misleading comparisons among Autism treatments. Finally, after compilation of the large data set from various intervention programs, we will be able to develop a three dimensional matrix of intervention components by child symptoms characteristics and behavior outcomes. With such a matrix, program managers, policy makers, teachers and/or parents will be able to identify empirically grounded interventions specifically related to target behavioral goals that may allow for a more rapid identification and implementation of an appropriate intervention.

To achieve the research objective of this dimension, the Dynamic Evaluation Model will collect (a) specialized demographic data and (b) standardized measurements such as GARS -3. At this point it is not important what specific measures will be used. What is important is that they will be standardized measures reliably measuring the changes in adaptive behavior and Autism symptoms.

The diagnostic measures for Specialized Autism Demographics will follow a groupcomposite: race, parents' education, parents' occupation, child medication, and when the child was first diagnosed. The diagnostic measures for Autism Symptoms Distribution produce groupcomposite, stereotyped behavior, communication, and social interaction scores in addition to the individual item scores. 
Table 3.1.

The Gilliam Autism Rating Scales: Stereotyped Behaviors

\begin{tabular}{cl}
\hline Item & \\
\hline 1 & Avoids eye contact/looks away \\
2 & Stares at hands, objects \\
3 & Flicks fingers rapidly \\
4 & Eats specific foods \\
5 & Licks, tastes, inedibles \\
6 & Smells/sniffs objects \\
7 & Whirls, turns in circles \\
8 & Spins objects \\
9 & Rocks back and forth \\
10 & Rapid lunging/darting \\
11 & Prances \\
12 & Flaps hands \\
13 & Makes high-pitches \\
14 & Slaps, hits, bites \\
\hline
\end{tabular}

Table 3.2.

The Gilliam Autism Rating Scales: Communication

\begin{tabular}{cl}
\hline Item & \\
\hline 15 & Repeats words \\
16 & Repeats out of context \\
17 & Repeats over and over \\
18 & Speaks/signs with flat tone/affect \\
19 & Responds inappropriately \\
20 & Looks away when called \\
21 & Does not ask for things \\
22 & Does not initiate conversation \\
23 & Uses "yes"' and "no" inappropriately \\
24 & Uses pronouns inappropriately \\
25 & Uses the word I inappropriately \\
26 & Repeats unintelligible sounds \\
27 & Uses gestures instead of speech \\
28 & Inappropriately answers about story \\
\hline
\end{tabular}


Table 3.3

The Gilliam Autism Rating Scales: Social Interaction

\begin{tabular}{cl}
\hline Item & \\
\hline 29 & Avoids eye contact \\
30 & Stares/looks unhappy when praised \\
31 & Resists physical contact \\
32 & Does not imitate \\
33 & Withdraws/remains aloof \\
34 & Unreasonably fearful \\
35 & Unaffectionate \\
36 & No recognition (looks through people) \\
37 & Laughs, giggles, cries inappropriately \\
38 & Uses toys/objects inappropriately \\
39 & Does things repetitively/ritualistically \\
40 & Upset when routines changed \\
41 & Tantrums when given commands \\
42 & Lines up objects, upset when disturbed \\
\hline
\end{tabular}

\section{Dimension 3: Environment}

I suggest that it is necessary to understand (a) in what ways the environment (e.g., family, school, and community) influences the intervention process and intervention outcomes and (b) in what ways the intervention process and outcomes influence the environment. Indeed, they are constantly mutually influencing each other. To capture the level of mutual influence, the Dynamic Evaluation Model will measure the interactions of the environment and the agent of change (complex intervention) and quantify the degree of influence each has with respect to the other. This will be accomplished via:

\section{The Impact of Environment on Treatment Process and Outcomes:}

1. The measurement of degree of family or service provider engagement

2. The measurement of the intervention outcomes (dimension 4) 


\section{The Impact of Treatment Process and Outcomes on Environment:}

1. The measurement of the degree of family or service provider stress

2. The measurement of the degree of satisfaction with the intervention ultimate outcomes

In addition, the Dynamic Evaluation Model will measure the context of the treatment implementation to understand further contextual factors impacting the results of the intervention. This will be accomplished via:

\section{The Impact of the Contextual Factors:}

1. The assessment of the treatment setting

2. The assessment of the treatment duration

3. The assessment of the treatment delivery format

4. The assessment of the treatment cost

5. The assessment of the treatment intensity

6. The assessment of the learners' background

The parent and staff surveys, Parenting Stress Index TX, Fourth Edition (PSITM - 4), Vineland Adaptive Behavior Scales, Second Edition (Vineland ${ }^{\mathrm{TM}}$ - II) [administered via dimension 4] will be the methodological tools to obtain the data with subsequent analyses of the results by items using measurements of central tendency, measurements of variability, correlations among items, and frequencies tables.

\section{Dimension 4: Outcomes}

I am assuming that the key result of behavioral intervention is to achieve some kind of change in some kind of behavior (e.g., improving communication, gaining/achieving eye contact, increasing social skills). I conceptualize behavioral change as a type of transition or transformation into desirable behavior. The degree of this change can uncover the actual capacity 
of the treatment. The actual capacity will be measured via assessment of the degree (pre and post) and the speed (time) of the changes in Autistic behavior.

This can be accomplished via quantitative standardized measurements such as Vineland Adaptive Behavior Scales, Second Edition (Vineland ${ }^{\mathrm{TM}}$ - II) and/or Preschool Language Scales, Fifth Edition (PLS $\left.{ }^{\mathrm{TM}}-5\right)$. Specifically, the outcome measures for the impact of the intervention produce group-composite, receptive language, expressive language, written language, personal, domestic, community, interpersonal relationship, play and leisure, coping skills, gross motor skills, and fine motor skills. 
Table 3.4.

The Vineland Adaptive Behavior Preschool Language Scales Composites

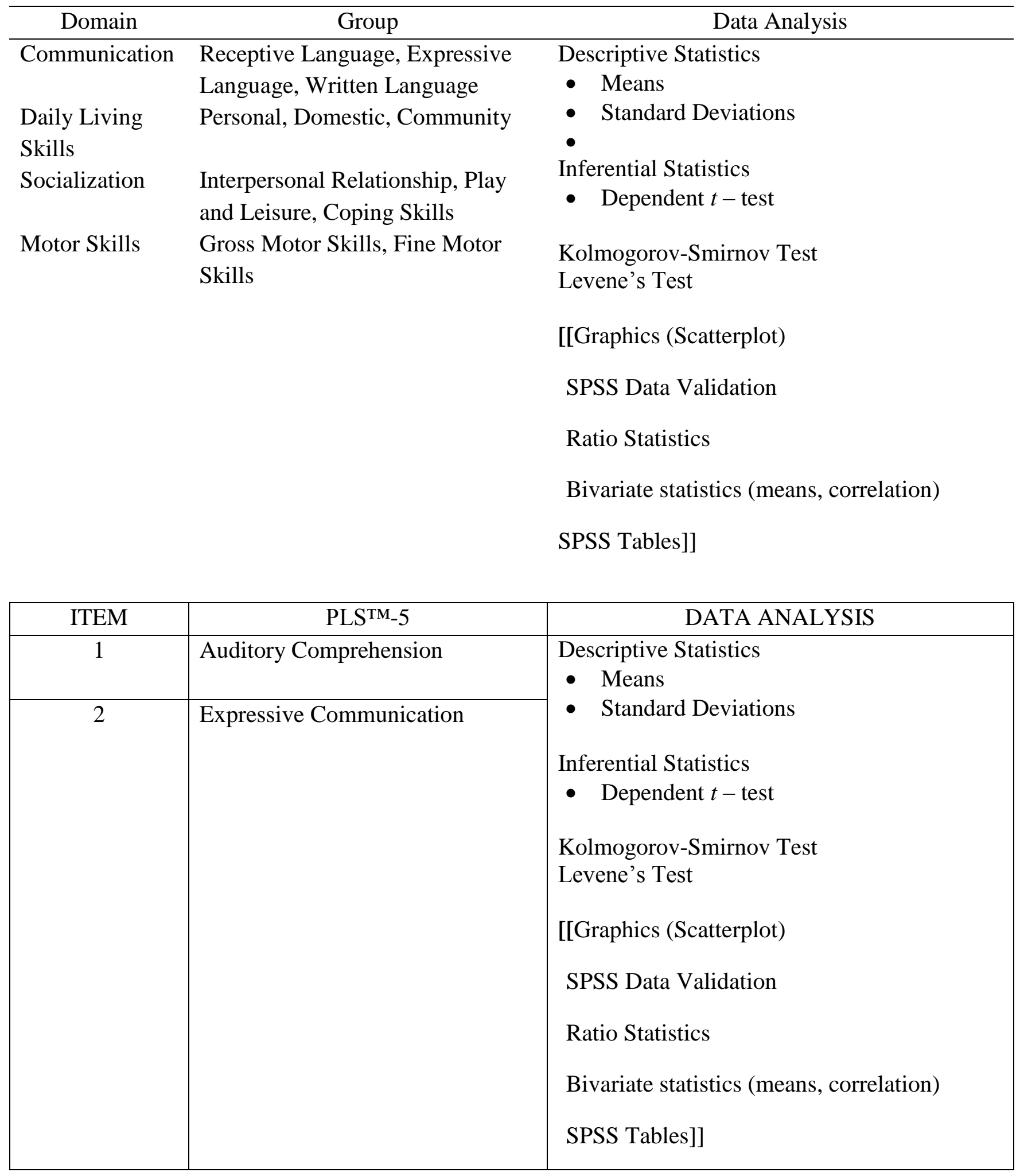




\section{Dimension 5: Understanding}

The objective of this phase is to bring understanding via integration of the data and integrative data analyses from all four Dynamic Evaluation Model Dimensions (D1- D4). Specifically, after implementation of the Dynamic Evaluation Model for evaluation of complex behavioral and other treatments for Autism we will:

Determine whether or not the treatment is effective (D4)

$>$ Determine the context of the treatment implementation (D3)

$>$ Understand who benefits the most from what particular intervention (D1, D2, D4) [Not currently known]

- Understand what programs are able to produce what outcomes (D1, D4)

$>$ Understand the speed (time) of the changes in behavior (D1, D4)

Understand the differences among treatments (D1)

$>$ Develop a three dimensional matrix of intervention components by child symptoms characteristic by behavior outcomes (D1, D2, D4)

$>$ Develop the Autism Symptoms Distribution [tabulation of symptoms their intensity and frequency] (D2)

$>$ Understand the Dynamics of Autism symptomology [the relationships among symptoms] (D2)

Contribute to the explanation of the phenomenon of differential responsiveness (D1, D2, D3, D4)

$>$ Understand the impact of the environment in the treatment process and outcomes (D3, D4)

$>$ Understand the impact of the treatment process and outcomes on environment (D3, D4)

$>$ Understand the impact of contextual factors (D3) 


\section{Scientific Significance}

I believe that application of the Dynamic Evaluation Model will lead to significant advances in our knowledge regarding Autism Spectrum Disorder. It will be evaluation research that may lead to understanding that can significantly contribute to the fields of Applied Behavior Analysis and Special Education. The application of the Dynamic Evaluation Model for evaluation of complex behavioral and other treatments for Autism will have multiple practical benefits and produce new knowledge about Autism Spectrum Disorder per se. The Dynamic Evaluation Model offers a systematic guide for comprehensive evaluation of complex interventions. The result of broader application (nationwide evaluation of Autism treatments) will enable professionals, parents, and other stakeholders to make informed decisions as to what kind of Autism treatment would be optimal for their child. Policy makers can be informed by the results of applications of the Dynamic Evaluation Model as to what kinds of policy changes need to take place to better and more efficiently serve clients. And fellow researchers of Autism may find the systematized and data based information valuable for their further research.

I tested the prototype of the Dynamic Evaluation Model on the Competent Learner Model (CLM), which is a complex behavioral program for children with Autism. I chose to test the prototype on the CLM because I had access to this intervention that was fully implemented at the Vista School in Pennsylvania and I had performed previous research regarding the CLM. The following chapters will present specific program evaluation for the CLM according to the DEM research design. 


\section{Chapter 4: Prototype of the Dynamic Evaluation Model at The Vista School:}

\section{Evaluated Complex Behavioral Program -The Competent Learner Model}

The Competent Learner Model (CLM) is a behavioral model derived from three independent yet interconnected theoretical foundations of Applied Behavior Analysis (ABA), Direct Instruction (DI), and Precision Teaching (PT). The main objective of the CLM is to assist teachers and/or parents while they endeavor to establish sets of behavioral skills that facilitate students' successful involvement in their home and/or school environments.

The CLM is a behavior-based instructive program with the objective to assist teachers and parents to: (a) systematically observe students' behavior; (b) gain understanding of behavioral dynamics; and (c) acquire skills and knowledge to regulate students' challenging behavior. The CLM Course of Study helps teachers and parents to attain the necessary skill sets and familiarity with the behavioral domain leading to teachers' and parents' effective management of behavioral misconduct. The development of the seven behavioral repertoires (observer, listener, talker, reader, writer, problem solver, and participator) is achieved by the use of trained teachers. These teachers and/or parents are taught via the CLM Course of Study to adeptly: (a) observe student behavior, (b) assess the behavior, (c) analyze the function of the behavior, and (d) know how and when to change the instructional conditions.

The developer of the CLM and others have suggested that, if teachers focus on creation of learning arrangements in concert with well-designed learning activities, then students' participation will be enhanced, leading to the development of competent learners (Warash, Curtis, Hursh \& Tucci, 2008). Accordingly, the CLM teaches how to structure learning environments so that students can achieve the best learning outcomes. The CLM's learning environments are changed from teacher-directed to semi-directed to peer-directed to non-directed 
so that the maximum development of the seven repertoires can be accomplished. At the end of the CLM Course of Study, teachers are able to recognize the function of students' behavior as well as replace undesirable student behavior with desirable behavior based on the development of the seven repertoires, which are first established, then strengthened, and finally maintained.

The CLM Course of Study is divided into 13 units and 4 sub-units with the following learning objectives:

Unit 0: Performs as Expected with Introductory CLM Unit

Unit 1: Assesses Learner's Performance Using CLRA Facts/Items

Unit 2: Delivers Lesson(s) to Develop CLRs Assessed

Unit 3: States Outcomes Factually \& Collects Reliable Data

Unit 4: Predicts the Likely Effect on a Learner's Repertoire

Unit 5: Monitors Participator Repertoires across Instructional Conditions

Unit 6: Determines What Stimuli Have Value

Unit 7: Conditions Stimuli as Reinforcers or to Have Value

Unit 8: Selects Lesson Placement for Validated Curricula

Unit 9A L1-3: Sets Up and Runs Teacher-directed Conditions for CLM Lessons 1-3 as suggested, yet is responsive to the effect of the current contingencies

Unit 9A L4: Sets Up and Runs Teacher-directed Conditions for CLM Lesson 4 as suggested, yet is responsive to the effect of the current contingencies.

Unit 9A L5: Sets Up and Runs Teacher-directed Conditions for CLM Lessons 5-7 as suggested, yet is responsive to the effect of the current contingencies.

Unit 9B: Sets Up and Runs Teacher-Directed Conditions

Unit 10: Determines the Source(s) of Reinforcement that Maintains Behaviors 


\section{Unit 11: Monitors Progress Data}

Unit 12: Delivers Supplementary Contingencies as Prescribed

The CLM Course of Study employs programmed instructions, guidelines, collaborative consultation, written handouts, and video examples to help teachers master the learning objectives. The mastery of learning objectives (Unit 0-Unit 12) and skills is demonstrated by passing performance checkouts with CLM coaches for each unit completed (Tucci, Hursh \& Laitinen, 2004). The function of Coaches is one of the most important facets of the CLM. The coaches:

Guide teachers through the CLM Course of Study

Provide written description for each lesson with learning objectives for the teachers and for the learners while particular repertoires are being developed

$>$ Aid teachers to assess learners' level of repertoire development

$>$ Encourage teachers to identify valued stimuli (reinforcers)

$>$ Aid teachers to appraise existing conditions for their learners in their learning environments

Support teachers to master learning objectives

Assist teachers to acquire Applied Behavioral Analysis skills via role-playing

Engage teachers in active discussions and problems solving

$>$ Gradually reduce the coaching support as teachers' skills and knowledge advance

The Coaches also set up coaching sessions to serve as a valued event and pair motivated teachers with less motivated teachers (Tucci \& Hursh, 1991).

In sum, the CLM teaches naïve learners (children without any or with only a few behavioral skills) via validated curricula to become Competent Learners (Tucci et al., 2004). The 
CLM employs systematic empirically-based assessment, instructional, and evaluation strategies (Tucci et al., 2004). The CLM offers ongoing instruction and coaching support to create and recreate instructional conditions that develop Competent Learner Repertoires (Warash et al., 2008).

In conclusion, for the reasons explained above and the very reason that the Competent Learner Model has little to no empirical evidence of its impact, effectiveness and social validation, I proposed to employ the following study. The purpose of this part of my dissertation study was to test the Dynamic Evaluation Model by exploring the effect of the Competent Learner Model on adaptive behavior, language development, and Autism symptoms of children with Autism Spectrum Disorder. I also investigated the effect of the Competent Learner Model on parental stress of parents of children with Autism.

\section{Method}

\section{Participants}

The first group of participants $(\mathrm{N}=107)$ was all children with Autism Spectrum Disorders who were or are students at The Vista School in Pennsylvania. The second group of participants $(\mathrm{N}=37)$ constituted parents and/or caregivers of the current students. The third group of participants $(\mathrm{N}=57)$ comprised teachers, administration and staff members of The Vista School. There were no exclusion criteria because the goal for this evaluation study was to get a maximum possible number, ideally including all the students, parents, teachers, administration and staff members at The Vista School.

\section{Students' Demographics}

Currently in 2014 The Vista School has $(\mathrm{N}=86)$ children with Autism Spectrum Disorders from Berks, Cumberland, Dauphin, Franklin, Juniata, Lancaster, Lebanon, Perry, and 
York Counties in Pennsylvania. The Vista School's students operate on the moderate to severe end of the autism spectrum. Specifically, the Vista School students exhibit severe delays in communication skills, demonstrate challenging behavior, require frequent assistance for activities of daily living, cannot engage in leisure activities, and require one-on-one instruction. The Vista School is a publicly funded facility and provides a safe environment. The students are 88.79\% male and $11.21 \%$ female. The students' $(\mathrm{N}=107)$ ethnicity follows: $75.70 \%$ are European Americans, $5.61 \%$ are African Americans, $1.87 \%$ are Hispanics, $1.87 \%$ are Asian Pacific Islanders, and $11.21 \%$ are others. Regarding the students' families' socio-economic status, $4.21 \%$ have a yearly income less than $\$ 20,000 ; 8.42 \%$ range from $\$ 20,000$ to $\$ 29,999$; $2.11 \%$ range from $\$ 30,000$ to $\$ 39,999 ; 2.11 \%$ range from $\$ 40,000$ to $\$ 49,000 ; 10.53 \%$ range from $\$ 50,000$ to $\$ 59,999 ; 6.32 \%$ range from $\$ 60,000$ to $\$ 69,000$ and $66.32 \%$ had yearly incomes of $\$ 70,000$ or more.

\section{Parents' Demographics:}

The Vista School fathers' primary ethnic identification is: $7.84 \%$ are African Americans, 73.53\% are European Americans, $0.98 \%$ are Asian Pacific Islanders, $0.98 \%$ are Native Americans, 3.92\% are Hispanics and 12.75\% are others. The Vista School mothers' primary ethnic identification is: 5.94\% are African Americans, $73.27 \%$ are European Americans, 2.97\% are Asian Pacific Islanders, $0.99 \%$ are Native Americans, $1.98 \%$ are Hispanics and $14.85 \%$ are others. The caregivers' marital status follows: $82.18 \%$ are married, $8.91 \%$ are divorced, $1.98 \%$ are widowed, $4,95 \%$ are separated and $1.98 \%$ have never been married.

Regarding education, the fathers' highest grade or year of school completed follows: $3.03 \%$ have completed grades 9 through 11, $16.16 \%$ have finished grade 12 or their GED, 25.25\% have some college or technical school, $30.30 \%$ have 4 years college and $25.25 \%$ have graduate 
education. The mothers' highest grade or year of school completed follows: $1.96 \%$ have grades 9 through $11.25 .49 \%$ have grade 12 or GED; $24.51 \%$ have some college or technical school; $21.57 \%$ have 4 years college, and $26.47 \%$ have graduate education. Regarding employment, the fathers' status of employment follows: $78.57 \%$ are employed for wages, $14.29 \%$ are selfemployed, $1.02 \%$ have been out of work for less than one year, 1.02\% are homemakers, $2.04 \%$ are retired, and $3.06 \%$ are unable to work. Mothers' status of employment follows: $41.41 \%$ are employed for wages, $10.10 \%$ are self-employed, $3.03 \%$ have been out of work for less than one year, $1.01 \%$ have been out of work for more than one year $40.40 \%$ are homemakers, $1.01 \%$ are retired and $3.03 \%$ are unable to work.

\section{Settings}

The study took place in two locations. The first was The Vista School, located at 1021 Springboard Drive, Hershey, Pennsylvania. The Vista School assists children with Autism Spectrum Disorders by creating a specialized educational environment. The Vista School was established in 2002 and at that time served only four children with Autism. Currently it serves 86 K-12 students. The Vista School describes itself as a school with an alternative, educational, and therapeutic program. The school serves children with Autism Spectrum Disorder with the mission to enhance independence of these children. The school's main objective is to help children to achieve an optimal amount of independence so that children can operate in their homes, schools, and communities. The Vista School obtained the Mental Health Partial Hospitalization certificate from the Pennsylvania Department of Public Welfare to operate its Educationally Integrated Behavioral Support Program. In this location all the data were collected. The Vista School personnel administered the standardized assessments because I do 
not have appropriate training and certification to do these types of specialized measurements. I administrated, collected and analyzed the data from two surveys.

The second location of this evaluation investigation was West Virginia University, where I managed, stored and analyzed the data. The West Virginia University (WVU) is a public land grant institution with Carnegie Foundation classification as "High Research Activity" and is accredited by the Higher Learning Commission of the North Central Association of Colleges and Schools. In addition, many West Virginia University programs have specialized accreditation. The WVU Board of Governors and the West Virginia Higher Education Policy Commission regulate the institution.

\section{IRB protocol}

This evaluation study was approved by the Institutional Review Board (IRB) and (a) was performed in an adequate public facility, (b) ensured the safety of all participants and (c) assured the confidentiality and privacy of all of the data. Two cover letters (see appendix) were distributed at the beginning of the data collection. I provided information about the study to all participants in a way that was understandable to them, and I encouraged participants to ask any questions in order to ensure that they understood the research objective and their role in it. The participants could withdraw any time from the study, or decline to answer any or all survey questions at any time during the research. I ensured that recruiting for this evaluation study was not coercive and made no false promises or claims. I did not use exculpatory language either in the written consent or in discussions about the research. I protected the rights and welfare of all participants at all times. 


\section{Procedures}

This evaluation study was performed in the following sequential order of steps:

1. The dissertation prospectus was written, presented and approved by my dissertation committee on May 20, 2013.

2. A protocol was written, presented and approved by the Institution Review Board with an expiration date of January 29, 2017.

3. All participants obtained a cover letter explaining the purpose of the study so as to make informed decisions about involvement in the study.

4. Participants were observed and measured via use of standardized measurements in their common educational activities at The Vista School, Pennsylvania. In this location all the assessment data were collected. The Vista School personnel administered the standardized assessments, specifically, The Preschool Language Rating Scale, Fifth Edition, The Gilliam Autism Rating Scale and The Vineland Adaptive Behavior Scales, Second Edition (Vineland -II) because I do not have appropriate training and certification to administer these measurements.

5. I developed, pilot tested, administered and analyzed the data from (a) parents' and/or caregivers 'anonymous electronic survey and (b) administration's and/or staff's anonymous electronic survey. I delivered the survey via Qualtrics on-line software.

6. I stored and analyzed the data at my office in Allan Hall at West Virginia University.

7. For the statistical analyses of the standardized measurements and the two surveys, I employed SPSS 21.0 (Statistical Package for the Social Sciences).

8. I will present the results of the evaluation study to: 
a. The Vista School's administration and staff members who will present the results to the parents and/or caregivers of their students

b. The Developer of the Competent Learner Model- complex behavioral program (Tucci Learning Solution)

c. My committee at my dissertation defense May 15, 2014, where I will discuss the limitations and the possible implications of this study.

d. I will prepare abbreviated manuscripts for publication and conference presentations.

\section{Measurement}

Because the purpose of the Dimension 4: Outcomes of the Dynamic Evaluation Model was measurement of the changes (if any) in behavior, social functioning and level of Autism symptoms, I employed three valid and reliable measures. Specifically, I employed (a) The Vineland Adaptive Behavior Scales, Second Edition (Vineland -II), (b) The Gilliam Autism Rating Scale: Second Edition (GARS-2), and (c) Preschool Language Scales, Fifth Edition (PLS $\left.{ }^{\mathrm{TM}}-5\right)$.

The Vineland Adaptive Behavior Scales. The Vineland Adaptive Behavior Scales, Second Edition (Vineland -II) was administrated via paper-and-pencil survey interview, and parent and teacher ratings forms. The standard scores for the adaptive behavior composite have $(M=100, S D=15)$ and the subdomains each have $(M=15, S D=3)$, percentile ranks, and adaptive levels. This assessment was designed for persons from birth to 90 years old, although it is commonly used to track and report progress for children with Autism Spectrum Disorders and other developmental disabilities. Specifically, the study measured the specific behavioral domains assessed by the adaptive behavior items. 
According to Sparrow and Balla (1984), the reliability is reported (Pearson) for internal consistency for survey form: split half means for domains 0.83 to 0.90 . For adaptive behavior composite 0.94 ; the expanded form: split half means for domains 0.91 to 0.95 ; for adaptive behavior composite 0.97 . Classroom edition: coefficient alpha means for domains 0.80 to 0.95 for adaptive behavior composite 0.98 . The reliability for the test-retest for survey form: means for domains 0.81 to 0.86 for Adaptive Behavior Composite $0.88(\mathrm{~N}=484)$. And the interrater reliability for survey form: correlations between two different interviewers; for domains 0.62 to 0.78; for adaptive behavior composite $0.74(\mathrm{~N}=160)$ (Sparrow \& Balla, 1984).

According to Sparrow and Balla (1984), the assessment's validity, specifically intercorrelations, was supported by correlations of domain standard scores, by age. The construct validity is supported by the developmental progressions of raw scores with age, the principal components analyses of domain standard scores, and the principal factor analyses of subdomain raw scores. The concurrent validity was tested by assessing the correlations between the Vineland and other adaptive behavior scales (Vineland Social Maturity Scale, Adaptive Behavior Inventory for Children, AAMD Adaptive Behavior Scale); and the correlations between the Vineland and tests of intelligence (K-ABC) and vocabulary (PPVT-R), (Sparrow \& Balla, 1984).

The Gilliam Autism Rating Scale, Second edition GARS-2т. Currently on the market is GARS-3, which was published at in 2013. This study used GARS-2 because GARS-3 was not available for two times at first; however, it but will be used in the future. The Gilliam Autism Rating Scale is a norm-referenced assessment to diagnose and classify children with Autism. This measurement is used for children between the ages of 3 to 22 .

The Gilliam Autism Rating Scale was normed on a representative sample of children with Autism (diagnosed according to DSM-IV) $(\mathrm{N}=1107)$ coming from 48 states in the United 
States. The Gilliam Autism Rating Scale is considered a strong psychometric measurement, which was validated and is reliable. The internal consistency of the Gilliam Autism Rating Scale was determined using Cronbach's Alpha with the coefficient of 0.84 for Stereotyped Behavior, 0.86 for Communication, 0.88 for Social Interaction, and 0.94 for the Autism Index. The measurement underwent several studies of validity and is considered valid (Multi-Health Systems Inc., 2013).

Preschool Language Scales, Fifth edition (PLS $S_{-5}^{\text {M. }) . ~ T h e ~ P r e s c h o o l ~ L a n g u a g e ~ S c a l e s ~}$ is a comprehensive language criterion based measurement. The Preschool Language Scales assess the development of language and was designed for birth to 7 years and 11 months. The assessment items range from pre-verbal, interaction-based skills to early literacy. The assessment is used for children who have Autism or severe developmental delays. For 6 - 7 year old children the assessment targets language skills based on Theory of Mind and assesses the use of irregular plurals, synonyms, and constructing sentences. The assessment has standardized scores and percentile ranks and measures two scales:

Within these two subscales it assess areas of attention to environment, attention to people, play, gesture, vocal development, social communication, semantics (vocabulary, qualitative concepts, quantitative concepts, spatial concepts time concept), language structure (morphology, syntax), integrative language skills, and emergent literacy skills. Regarding psychometric information the Preschool Language Scales was tested on $(\mathrm{N}=1400)$ children in a normative sample coming form 45 states from the United States. Clinical studies also included a developmental delay study and three types of language disorders (children with receptive language disorder, expressive language disorder, and both receptive and expressive 
The Parenting Stress Index Short Form. Has 36 items measuring five subscales of Defensive Responding, Parental Distress, Parent-Child Dysfunctional Interaction, Difficult Child and Total Stress

Defensive Responding. The parents' Defensive Responding subscale is measuring responder tendency to minimize the stress in parent-child relationship. Scores of 10 or lower are considering as extremely defensive and can lead to invalid results.

Parental Distress. The parental distress subscale assessed the degree of stress that a parent feels as a consequence of his/her role via measurement of the parent competence, stress associated with restrictions on his/her life, social support and depression (PSI- SF, Guidance Document).

Parent-Child Dysfunctional Interaction. This subscale measures if the child meets the parental expectation and the satisfaction of the child parent interaction (PSI- SF, Guidance Document).

Difficult Child. This subscale measures parental perception of easy or difficult child (behavioral characteristics of the child) that influence parent-child relationship (PSI- SF, Guidance Document)

Total Parental Stress. This subscale measures the overall level of parental stress. The measure does not assessed the life distress rather only stress related to the parenting (PSI- SF, Guidance Document).

Measurement of satisfaction with the CLM ultimate outcomes. Parents and/or caregivers measured the degree of satisfaction via anonymous survey with the CLM Ultimate Outcomes. The degree of satisfaction was calculated based on 7 levels Likert Scale where $1=$ 
Strongly Agree, 2 = Agree, $3=$ Somewhat Agree, $4=$ Neutral, $5=$ Somewhat Disagree, $6=$ Disagree, and $7=$ Strongly Disagree .

Measurement of parents and/or caregivers satisfaction with The Vista School. Parents and/or caregivers measured the degree of satisfaction via anonymous survey with The Vista School. The degree of satisfaction was calculated based on 7 levels Likert Scale where $1=$ Strongly Agree, $2=$ Agree, $3=$ Somewhat Agree, $4=$ Neutral, $5=$ Somewhat Disagree, $6=$ Disagree, and $7=$ Strongly Disagree .

Measurement of administration and staff satisfaction with the CLM ultimate outcomes. Administration, staff and teachers of The Vista School measured the degree of satisfaction with CLM Ultimate Outcomes via anonymous survey. The degree of satisfaction was calculated based on 7 levels Likert Scale where 1 = Strongly Agree, 2 = Agree, $3=$ Somewhat Agree, $4=$ Neutral, $5=$ Somewhat Disagree, $6=$ Disagree, and $7=$ Strongly Disagree.

Measurement of teachers, administration and/or staff satisfaction with other components of the CLM. Administration, staff and teachers measured the degree of satisfaction with components of CLM via anonymous survey with The Vista School. The degree of satisfaction was calculated based on 7 levels Likert Scale where $1=$ Strongly Agree, $2=$ Agree, $3=$ Somewhat Agree, $4=$ Neutral, $5=$ Somewhat Disagree, $6=$ Disagree, and $7=$ Strongly Disagree.

Measurement of autism specialized demographics. Parents and/or caregivers measured the parents' level and type of education, children medical/ pharmacological treatments and other Autism Treatments via anonymous survey items. 


\section{Research Design}

This evaluation study employed the prototype of the Dynamic Evaluation Model (DEM) for the comprehensive evaluation of the Competent Learner Model (CLM) at The Vista School (see appendix table A3/7 for graphic representation of the CLM evaluation according DEM). The research questions (objectives) derived from the Dynamic Evaluation Model.

\section{DEM Dimension 1: Conceptualization}

The research objective of this phase of the DEM was to determinate the Competent Lerner Model constellation. For this purpose I communicated with the developer of the program (Vicci Tucci) who answered the follow questions:

$>$ What are the intervention parts?

$>$ How many parts the intervention has?

$>$ What is the intensity of intervention (time)?

$>$ What are the functional relations among the parts?

What are the strategies?

$>$ What are the intervention assumptions?

What are the intervention short-term outcomes?

What are the intervention intermediate outcomes?

$>$ What are the intervention ultimate outcomes?

\section{DEM Dimension 2: Diagnostics}

The research objective of this phase of the DEM was to collect (a) specialized demographic data and (b) to uncover the "Autism Symptoms Distribution" to develop a tabulation of Autism symptoms. (See table 3.1-3.3) This was done via quantitative standardized 
measurement GARS-3 and surveys items by methods of measurements of central tendency, measurements of variability and development of the frequencies tables. Specifically, the diagnostics measures for specialized demographics had follow group-composite: race, parents' education, parents' occupation, medication, and when the child was first diagnosed. The diagnostics measures for Autism Symptoms Distribution had follow group-composite: stereotyped behavior, communication and social interaction.

\section{DEM Dimension 3: Environment}

The research objective of this phase of the DEM was to collect data to assess the contextual factors via cross sectional survey and by methods of measurements of central tendency, measurements of variability, correlations coefficients and development of the frequencies tables. To capture the level of mutual influence the Dynamic Evaluation Model measured the interactions of the environment and the agent of change (complex intervention) and quantified the degree of influence each has with respect to the other. This will be accomplished via:

\section{The Impact of Environment on Treatment Process and Outcomes:}

1. The measurement of degree of family or service provider engagement

2. The measurement of the intervention outcomes (dimension 4)

\section{The Impact of Treatment Process and Outcomes on Environment:}

1. The measurement of the degree of family or service provider stress

2. The measurement of the degree of satisfaction with the intervention ultimate outcomes

In addition, I planned via the Dynamic Evaluation Model to measure the context of the treatment implementation to understand further contextual factors impacting the results of the intervention. This was to be accomplished via: 


\section{The Impact of the Contextual Factors:}

1. The assessment of the treatment setting

2. The assessment of the treatment duration

3. The assessment of the treatment delivery format

4. The assessment of the treatment cost

5. The assessment of the treatment intensity

6. The assessment of the learners' background

\section{DEM Dimension 4: Outcomes}

The research objective of this phase of the DEM was to collect data to determinate the actual capacity (assess the impact) of the Competent Learner Model. Specially, to uncover the degree, form and the speed of the changes in adaptive behavior. This was done via quantitative standardized measurement Vineland-II and PLSTM -5 by methods of measurements central tendency, measurements of variability, inferential and statics (depended $t$-test). Specifically, the outcomes measures for the impact of the intervention had a follow group-composite: Receptive Language, Expressive Language, Written Language, Personal, Domestic, Community, Interpersonal Relationship, Play and Leisure, Coping Skills, Gross Motor Skills, and Fine Motor Skills. (See table 3.4.)

\section{DEM Dimension 5: Understanding}

The research objective of this phase of the DEM was to expand our knowledge about Autism, provide a contextual understanding of CLM effectiveness, identify who benefits the most from the intervention, identify which children with what kind of symptoms' characteristics can benefit the most from the CLM structure, create Autism Symptoms Distribution for The 
Vista School Pennsylvania and the Dynamics of Autism Symptomology [the relationships among symptoms] at The Vista School Pennsylvania.

\section{Data Analyses}

I will address the type of data analyses that I performed for each dimension of the Dynamic Evaluation Model individually.

\section{Dimension 1: Conceptualization}

For this dimension no statistical analyses were performed. I asked the developer of the Competent Learner Model to answer the questions in order to develop the Structural Framework of the Competent Learner Model. I obtained some answers (see results in chapter 6, Dimension 1), however not enough detail was provided to construct the complete Structural Framework of the Competent Learner Model as a complex behavioral intervention.

\section{Examination of Assumptions}

I identified the theoretical assumptions of the Competent Lerner model. (See chapter 6dimension 1)

\section{Dimension 2: Diagnostics}

For this dimension of the evaluation I performed statistical analyses to understand the symptoms representation. Specifically, descriptive analyses of measurement of central tendency and measurement of the variability were calculated. In addition, graphs were used to see the trend in the data. However, I was not able to develop the Autism Symptoms Distribution (the data I collected is only valid for the current Vista School students) because I only obtained the subscales raw score and the Autism Index. For developing the detailed symptoms tabulation for the Vista School I would need to perform the statistical analyses on the individual items levels because each item represents one specific Autism symptom. 


\section{Examination of Assumptions}

No specific assumptions for the descriptive analyses were evaluated.

\section{Computation of the Tests Statistics}

The frequency table of the medication type was developed and the sample mean $\mathrm{n}$ was calculated to obtain the mean values for Stereotyped Behavior Symptoms, Communication and Social Interaction.

The sample standard deviation was calculated to understand the variability among the symptoms representation. The percentages were used to see the proportion in gender, race, and socio economic status of the parents. Frequencies Tables were planned to construct to see how many times each symptoms value occurred in the data.

\section{Dimension 3: Environment}

For this dimension of the evaluation I performed statistical analyses to assess environmental factors. Specifically, descriptive analyses of measurement of central tendency and measurement of the variability were calculated. In addition, I employed bivariate statistics to measure the relationship between some survey items, (see chapter 6) thus I calculated the Pearson product-moment correlation. According to the Dynamic Evaluation Model I was also suppose to calculate the impact of the environment on the treatment process and treatment outcomes via measurement of the degree of family or service provider engagement. This was not completed because by the time this measure (survey of family involvement) was developed the other surveys were already IRB approved and administrated. For the measurement of the degree of satisfaction with the Competent Learner Model and The Vista School and the measurement of the degree of family stress I employed the descriptive analyses of measurement of central tendency and measurement of the variability with the results of these surveys. 


\section{Examination of Assumptions}

No specific assumptions for the descriptive analyses were evaluated.

\section{Setting the Level of Significance}

For the correlation the level of significance of risk of Type I error was set on $p<0.05$.

\section{Computation of the Tests Statistics}

The sample mean was calculated to obtain the mean values for and the sample standard deviation was calculated and the Pearson product-moment correlation.

\section{Dimension 4: Outcomes}

For this dimension of the evaluation I performed statistical analyses to assess what is the impact, and magnitude of the impact, of the Competent Learner Model on the development of Children with Autism Spectrum Disorders at Vista School. Specifically, descriptive analyses of measurement of central tendency and measurement of the variability were calculated and the inferential statistics particularly dependent $t$ - test was employed on some of the measured outcomes and on others the Wilcoxon signed-rank test (see assumptions examination below) to quantify the changes in measured behavior.

\section{Examination of Assumptions}

The assumptions of the two dependent samples, comparison made on same trait, ratio levels of measurement were met but the assumption of normality presented a statistical judgment call. Because I employed paired-sample $t$ - test I analyzed the sampling distributions of the differences between scores rather than normality of the data per se. For this purpose I employed the Kolmogorov-Smirnoff test. In the majority $(\mathrm{N}=20)$ of the compared pairs I did find that the sampling distributions of the difference were not normally distributed (see appendix table X). However, for the vast majority of the pair comparisons $(\mathrm{N}=24)$ the sample size was large. Only 
five pairs had a relatively small sample size but paradoxically (see explanation below) four of these distributions, according to Kolmogorov-Smirnoff test, were normally distributed.

I also constructed the Q-Q plots for each distribution (see appendix, figure) to visually judge how much the quantiles fall beyond the diagonal line representing the normal distribution. I employed this additional test because (1) the Kolmogorov-Smirnoff test is extremely sensitive to just a little deviation from normality in a large sample size which then leads to significant findings and thus a wrong assumption that the normality assumption was not met. And vise versa the Kolmogorov-Smirnoff test does not have the power to uncover the not normally distributed data in a small sample size which can lead to wrong assumptions that the data are normally distributed while they are not. (2) In addition, given the phenomenon of the Central Limit Theorem where the distribution is consider normal (and normality tests are not need it) regardless how the scores look when the sample size is large.

After evaluation of the Q-Q plots for each distribution I decided that in almost each distribution there was some degree of problem with kurtosis because the dots were either above or below the line or both indicating slight skew. There were also a good number of outliers further skewing the distributions from the normality. However, given the fact that $t$-tests, particularly dependent $t$-test, are robust enough to handle some amount of distribution irregularity I employed parametric depended $t$-test but on the 4 pairs comparisons that had more severe problems with Q-Q plot I employed the nonparametric equivalent to a pair $t$-test Wilcoxon signed-rank test on distribution that which Q-Q plot indicated larger problem with deviation from normal distribution.

Specifically, for the non-normally distributed distributions D18 (Motor Skill), D19 (Gilliam Autism Index), D23 (Auditory Comprehension) and D29 (Total Stress) see graphs 
below (figures 5.1, 5.2, 5.3, and 5.4) representing the pairs comparisons of Vineland measurement of motor skill domain, Gilliam measurement of Autism index, Preschool Language Scale measurement of auditory comprehension and the Parenting Stress Index measurement of total stress the nonparametric equivalent to a pair $t$-test Wilcoxon signed-rank test was employed.

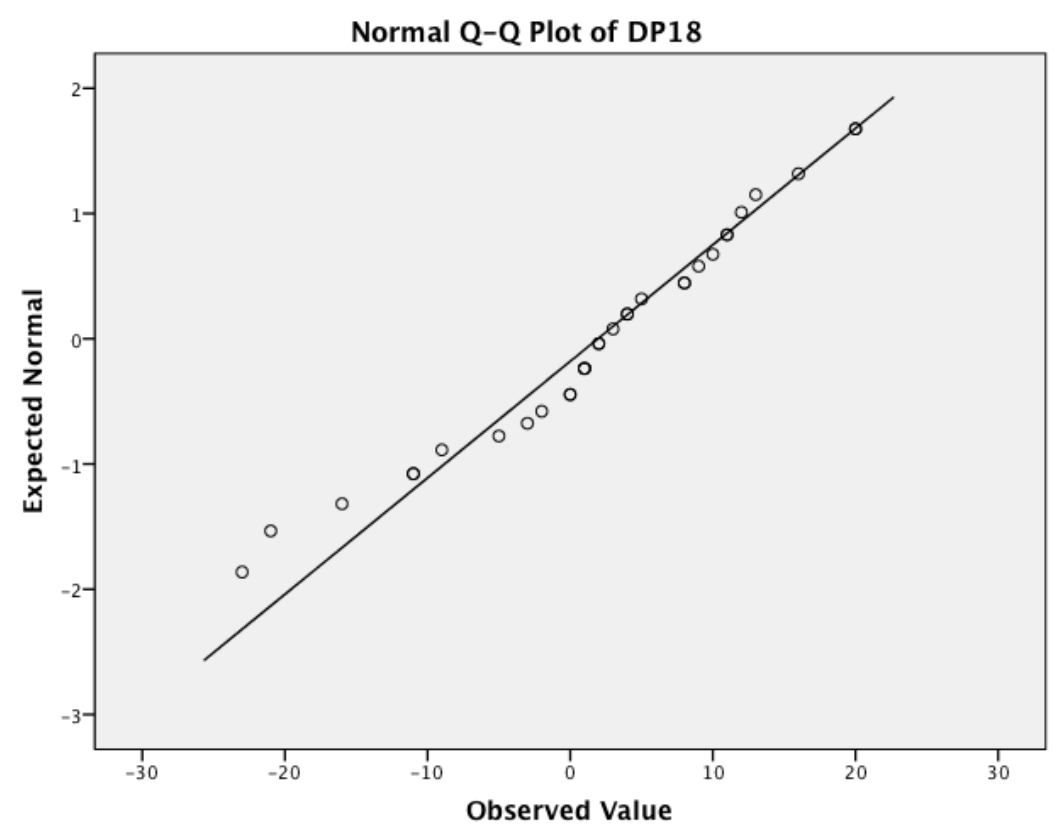

Figure 4.1. Normal Q-Q Plot for distribution D18 (Motor Skills). Figure is illustrating Exanimation of Normality Assumption 


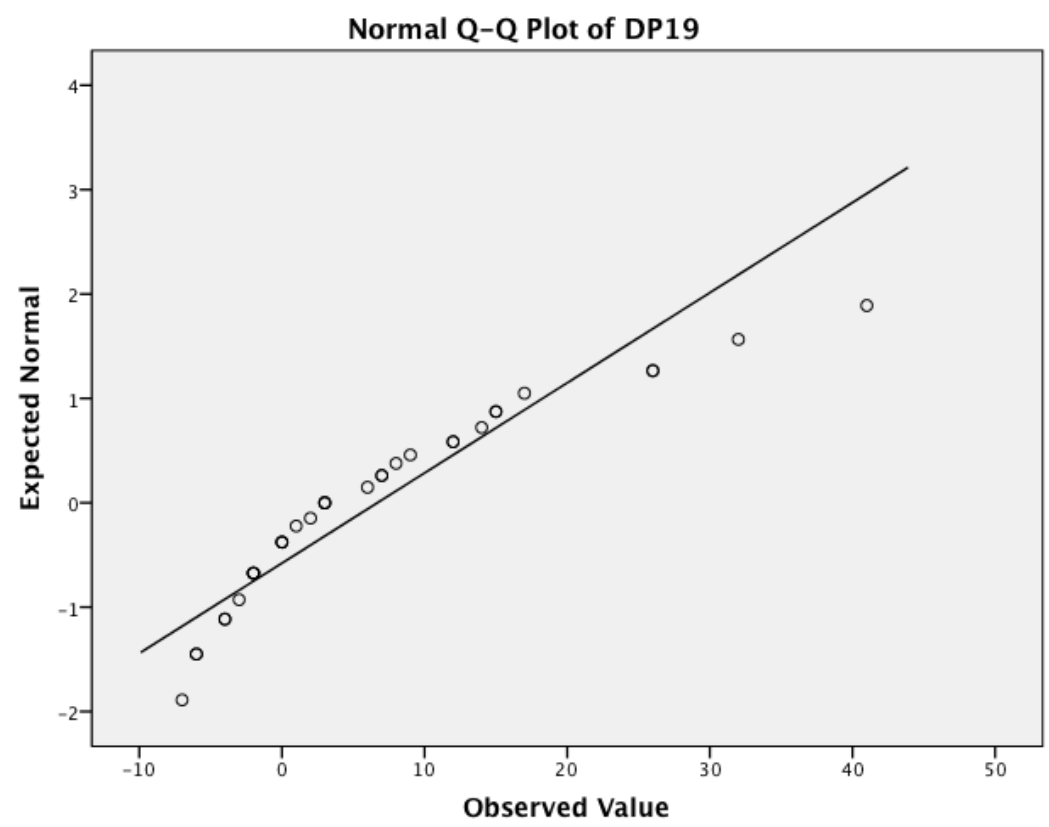

Figure 4.2. Normal Q-Q Plot for distribution D19 (Gilliam Autism Index). Figure is illustrating Exanimation of Normality Assumption

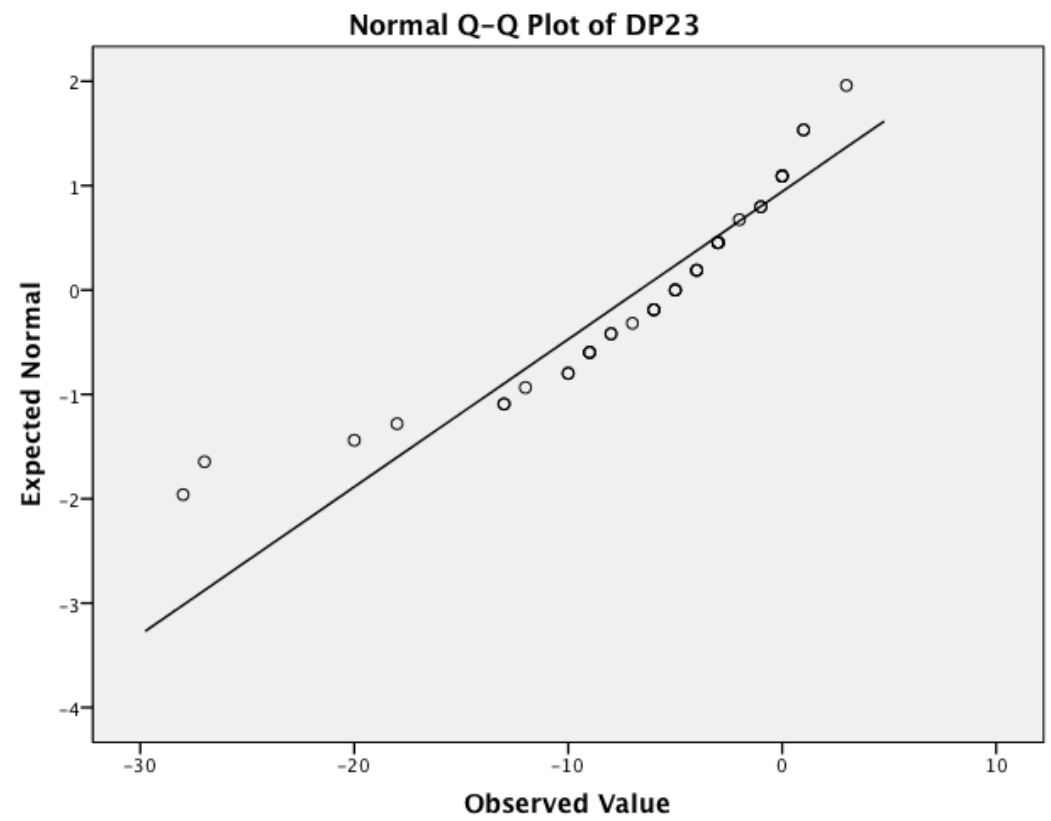

Figure 4.3. Normal Q-Q Plot for distribution D23 (Auditory Comprehension). Figure is illustrating Exanimation of Normality Assumption 


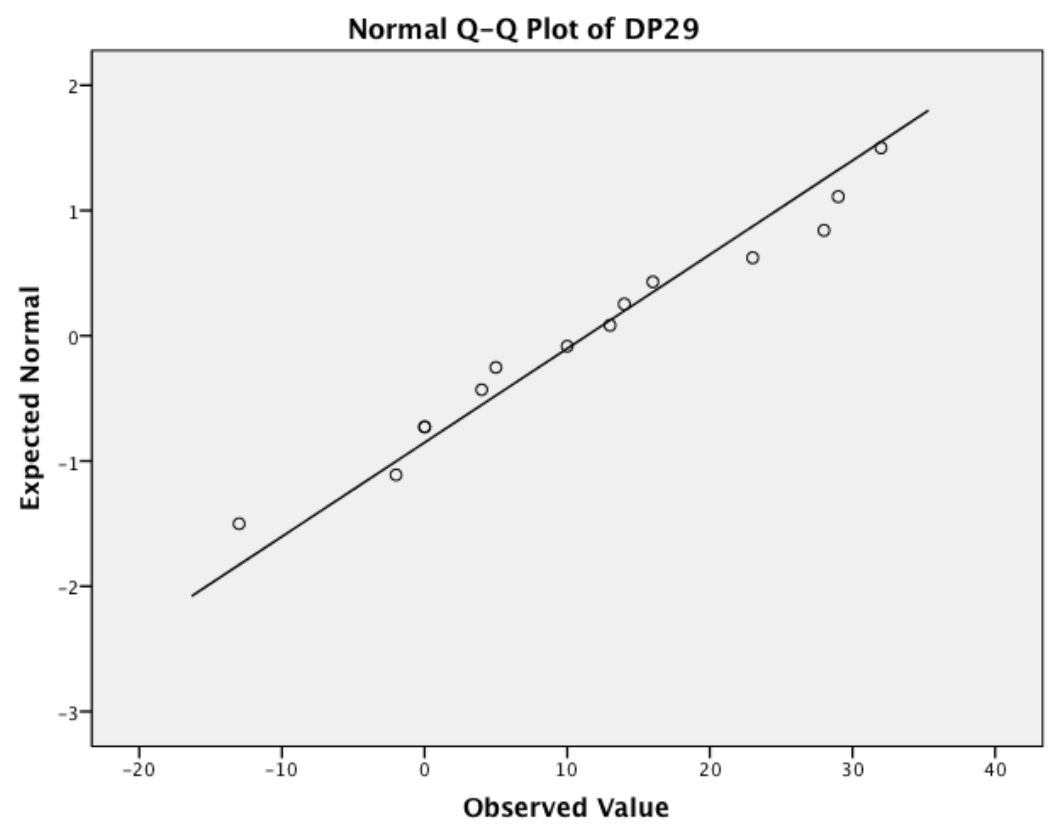

Figure 4.4. Normal Q-Q Plot for distribution D29 (Total Stress). Figure is illustrating Exanimation of Normality Assumption

\section{Research Hypothesis}

(1) There will be difference in the measured adaptive behavior domains, measured by the Vineland Adaptive Behavior, from before the CLM implementation to after CLM implementation.

(2) There will be difference in the measured language scale, measured by the Preschool Language Scale, from before the CLM implementation to after CLM implementation.

(3) There will be difference in the autistic symptoms, measured by the Gilliam Autism Rating Scales, from before the CLM implementation to after CLM implementation.

(4) There will be difference in the stress level of The Vista School students' parents, measured by the Parenting Stress Index, from before the CLM implementation to after CLM implementation. 


\section{Setting the Level of Significance}

At the beginning of this study I was thinking to pose a positive directional research hypotheses because I was expecting positive changes in the behavior due to the Competent Learner Model intervention. However, after careful evaluation of the assumption of the normality and given the fact the distributions are not perfectly normal I have decided to be more conservative and posed non directional research hypotheses, thus I performed the two tailed test and set the level of significant on 0.05 .

\section{Computation of the Tests Statistics}

The sample means for each pair comparisons were calculated to obtain the mean values. The standard deviations for each pair comparisons were calculated. The parametric $t$-test for majority of the pair comparisons was calculated.

The Wilcoxon signed-rank test for the pair comparisons of Vineland measurement of motor skill domain, Gilliam measurement of Autism index, Preschool Language Scale measurement of auditory comprehension and the Parenting Stress Index measurement of total stress were calculated.

The Cohen's $d$ was used to calculate effect sizes given the tendency of overestimation of the population effect size when using $t$ from paired samples while computing $r$. 


\section{Chapter 5: Results}

This evaluation study employed the prototype of the Dynamic Evaluation Model (DEM) for the comprehensive evaluation of the Competent Learner Model at The Vista School. The research questions (objectives) derived from the Dynamic Evaluation Model (DEM).

\section{Results from DEM Dimension 1: Conceptualization}

The Competent Learner Model (CLM) is constructed from the follow parts: (1) Online Electronic CLM Performance Tools and Face-to-Face Assistance in Actual Settings with Various Team Members, (2) Course of Study for Educators/Parents, (3) Coaching for Educators/Parents, Curricula for Learners, (4) Performance Assessments for Educators/Parents, (5) Performance Assessments for Learners, (6) Collaborative Consultations with Educators/Parents and (7) Action Management for Educators/Parents. These parts are related by means of the following strategies:

(1) Online Courses, Cloud-based Google Docs, and Responsive Management, (2), Approaches (i.e., resources provided to perform expected outcomes for each Team Member), (3) ABA (Applied Behavior Analysis), (4) DI (Direct Instruction), (5) PT (Precision Teaching) and (6) PSI (Personalized System of Instruction)

\section{CLM Assumptions}

The Competent Learner Model depends on several general assumptions about human behavior. Explicitly, the Competent Learner Model operates on positivist epistemological beliefs. Specifically, the Competent Learner Model assumes that the universe is a lawful and orderly place that can therefore be studied by using objective scientific methods. The Competent Learner Model assumes that teachers' and students' behavior and the environmental events contiguous with that behavior can and should be objectively observed, described, and quantified via systematic and objective (reliable) data collection so that teachers' and students' behavior 
can be established, strengthened, and maintained or weakened in socially validated directions. It presumes that human behavior is constantly changing but can be analyzed and modified by finding the functional relations among teacher behavior, student behavior, and other environmental events. The Competent Learner Model postulates that in order for students' personal, social, and academic behavior to be maintained in everyday circumstances, a set of seven behavioral repertoires must be established, strengthened, and maintained. The Competent Learner Model suggests that development of the seven Competent Learner Repertoires (observer, listener, talker, reader, writer, problem solver, and participator) will result in appropriate personal, social, and academic functioning of students with Autism in everyday circumstances.

\section{CLM Short-Term Outcomes}

1) Teachers will acquire $\mathrm{ABA}$ knowledge to understand behavioral principles

2) Teachers will correctly assess students' performance

3) Teachers will collect reliable data about students behavior

4) Teachers will determine sources of valuable reinforcement

5) Teachers will deliver effective reinforcement

\section{CLM Intermediate Outcomes}

1) Teachers will acquire ABA skills to develop the 7 learner repertoires (7Rs)

2) Teachers will deliver lessons to develop the $7 \mathrm{Rs}$

3) Teachers will predict students' performance

4) Teachers will routinely monitor students' participation across different instructional conditions

5) Teachers will arrange and re-arrange instructional conditions to discover the functional relations between behavior and environment 


\section{CLM Long-Term Outcomes}

1) Instructors (e.g., parents and educators) use empirically validated behavioral and instructional practices systematically to develop competent learners who can function successfully in new day-to-day situations.

2) Naïve learners become more and more competent learners who can act effectively to produce their desired outcomes or the expected outcomes of others in new situations without being explicitly taught by an instructor.

3) Instructors develop mastery of the evidence-based practices (i.e., formulate, deliver, and monitor CLM behavioral and instructional programming) at their own pace via the online course of study.

4) Instructors apply what they have learned in each of the CLM Course of Study Units either in person or via Virtual Coaching.

5) Scoped and Sequenced CLM Lessons are delivered to establish, strengthen, and maintain the competent learner repertoires (e.g., Talker, Problem Solver, and Participator)

6) Team Members (i.e., Instructor, Coach, Coordinator, and Administrator) conduct on-going self-assessments of the expected outcomes they are to exhibit during the implementation of CLM Programming

7) Team determines the "missing" Competent Learner Repertoires (CLRs) and then monitor the progress of the development of the CLRs

8) Team makes conspicuous the effects of the existing procedures in place in the setting (i.e., learning environment) for the educator and/or parent. Thereby they determine collaboratively the changes that need to be made for the development of the CLRs and the weakening of undesirable repertoires (e.g., Injurious Behaviors). 
9) A facilitator conducts action management planning sessions to delineate the results and concerns of the parents and/or educators to determine the learners' ongoing objectives

\section{CLM Ultimate Outcomes}

The ultimate goal of the Competent Learner Model is that students with Autism will develop the seven Competent Learner Repertoires (observer, listener, talker, reader, writer, problem solver, and participator) to the degree that they are maintained over prolonged periods of time and thus allow the students to engage in everyday functional actions. These repertoires are developed gradually by instructional formats organized into Competent Learner Model Curriculum lessons with a specific scope and sequence. The general learning objective for each repertoire follows:

1) The goal for the talker repertoire is that the student with Autism accurately and fluently uses sounds, words, and detailed statements about actions and objects and answers questions when and where appropriate.

2) The goal for the listener repertoire is that the student with Autism accurately and fluently follows instructions and uses rules when and where appropriate.

3) The goal for the observer repertoire is that the student with Autism accurately and fluently matches objects, sorts objects, and imitates actions when and where appropriate.

4) The goal for the reader repertoire is that the student with Autism accurately and fluently says written words and answers questions about what was read.

5) The goal for the writer repertoire is that the student with Autism accurately and fluently copies, takes dictation, and independently produces text that has the appropriate effect on the reader of that text. 
6) The goal for the problem solver repertoire is that the student with Autism will accurately, fluently, and appropriately ask for help, items, or actions, ask about the actions of others or events, say no when offered something not preferred and tell what is preferred instead, and manipulate items to make them work or to gain access to something preferred.

7) The goal for the participator repertoire is that the student with Autism engages in actions as directed by teachers, other adults, or peers, or that are appropriate for completing assignments, working independently, or using free time to do what they want.

To illustrate these goals, I developed a Theory of Change (see figure below) of the Competent Learner Model. 


\section{Theory of Change}

The Competent Leaner $\underline{\text { Model }}$

The Competent Leaner Model implements multiple strategies to develop the knowledge and skills that teachers need to effectively assist students with Autism to

develop $7 \mathrm{R}^{1}$

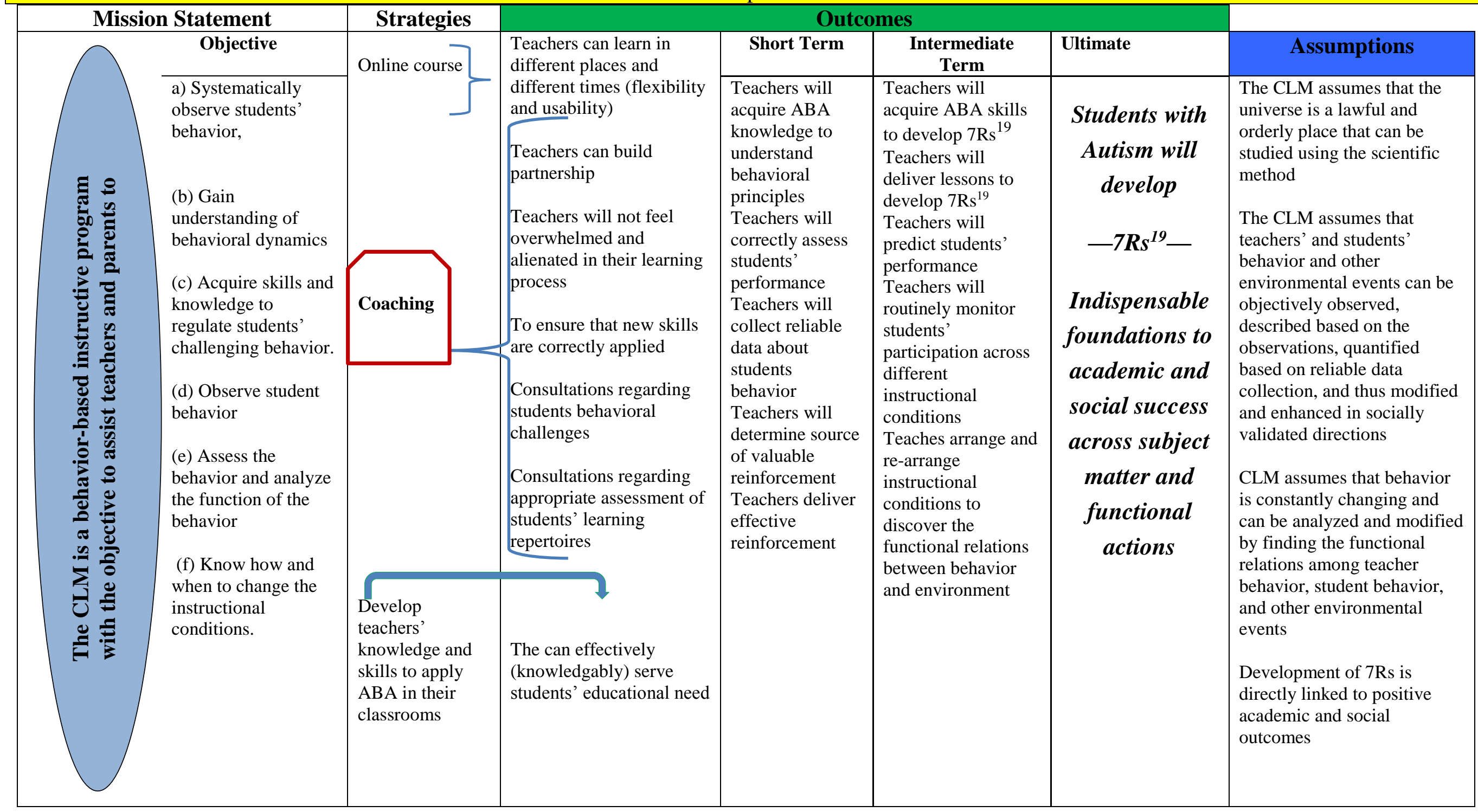

\footnotetext{
${ }^{19}$ 7R: Seven CLM Behavioral Repertoires (observer, listener, talker, reader, writer, problem solver, participator)
} 


\section{Results from DEM Dimension 2: Diagnostics}

\section{Autism Symptoms Distribution}

I developed a general tabulation of the symptoms distribution at The Vista School. Specifically, for the evaluation of the Competent Learner Model at The Vista School, I was able to develop a simple general tabulation of symptoms (see table 5.1 below). According to the Autism Index Scale, a person scoring 85 and above is classified by GARS-2, as very likely autistic (high-level of Autism). An Autism Index score from 70-84 indicates a person who is possibly autistic. An Autism Index measure of 69 or less indicates a person who is unlikely to be autistic. At the Vista School, the students who began the treatment were all very likely Autistic (high-level of Autism) on the overall Autism Index. The subscales of stereotyped behavior, communication and social interaction are interpreted, according to GARS-2, as very likely autistic with a score of 7 or higher. Scores from 4- 6 indicate a probability of Autism and scores of 1 to 3 are interpreted as suggesting an unlikely probability of Autism. Communicating with repeated words, responding inappropriately etc., were the most impacted by Autism ( $\mathrm{M}=9.10$, $\mathrm{SD}=2.77$ ). Social interaction such as showing no recognition of a person's presence or becoming upset when routines are changed was less impacted by Autism than communication, but also in the category of very likely probability of Autism $(\mathrm{M}=8.43, \mathrm{SD}=2.93)$. And finally the stereotypical behaviors such as avoiding eye contact, staring at hands, rapidly lunging, were the least impacted by Autism, however still in the very likely probability of Autism category $(\mathrm{M}=8.34, \mathrm{SD}=2.69)$. This simple general tabulation of the Autism Symptoms Distribution at the Vista School clearly indicates that students at The Vista School are very Autistic in all measured subscales. 
Table 5.1

Autism Symptoms Distribution at The Vista School Simple General Tabulation

\begin{tabular}{lcc}
\hline \multicolumn{1}{c}{ Gilliam Autism Rating Scale-Second Edition } & Mean & SD \\
\hline Stereotyped Behavior & 8.34 & 2.69 \\
Social Interaction & 8.43 & 2.93 \\
Communication & 9.10 & 2.77 \\
\hline \hline Autism Index & 91.43 & 13.07 \\
\hline
\end{tabular}

\section{Autism Symptoms Dynamics}

Regarding the symptom dynamics at The Vista School (see table 5.2 below), not surprisingly, there were significant large and positive relationships between the Autism Index and Stereotyped Behavior, $r=.69, p=.001$; the Autism Index and Communication, $r=.60, p=$ .001 ; and the Autism Index and Social Interaction, $r=.76, p=.001$. Somewhat unexpectedly, there were statistically not significant small positive correlations between Communication and Social Interaction, $r=.28, p=.091$, and Communication and Stereotyped Behaviors, $r=.13, p$ $=.404$. Finally, there was a significant but small positive relationship between Stereotyped Behavior and Social Interaction, $r=.38, p=004$.

Table 5.2

Autism Symptoms Dynamics at The Vista School

\begin{tabular}{lcccc}
\hline & Autism Index & $\begin{array}{l}\text { Stereotyped } \\
\text { Behavior }\end{array}$ & Communication & Social Interaction \\
\hline Autism Index & - & - & & \\
Stereotyped Behavior & $.69^{* *}$ & .13 & - & .27 \\
Communication & $.60^{* *}$ & $.38^{* *}$ & - \\
Social Interaction & $.76^{* *}$ & &
\end{tabular}




\section{Autism Symptoms and Treatment Outcomes Dynamics}

I developed a correlation matrix (see table 5.3, below) that revealed a pattern in the dynamics between the students' symptoms, measured by the Gilliam Autism Rating Scale, and the Competent Learner treatment outcomes measured by The Vineland Adaptive Behavior Scales. The correlation matrix clearly indicates that the Competent Learner Model almost exclusively (but one pair of correlation) significantly negatively correlates (the more adaptive behavior is developed the less Autism characteristic is present) between many adaptive behavior subscales and mitigating symptoms of stereotyped behaviors in children with high-level Autism.

Specifically, among all the 72 correlations coefficients measuring the relationship between the Competent Learner treatment outcomes and the Autism symptoms, there was only one significant correlation between Receptive Language and Autism Index where Receptive Language was significantly related to the decreasing of the Autism Index, $r=-.42, p=.15$. The rest of the significant relationships were "only" in the stereotyped behaviors. Particularly, the increasing of the Adaptive Behavior outcome was significantly related to the decreasing of the Stereotyped Behaviors symptoms, $r=-51, p=.003$. The increasing of the Receptive Language outcome was significantly related to the decreasing of the Stereotyped Behaviors symptoms, $r=$ $-.52, p=.002$. The increasing of the Written Language outcome was significantly related to the decreasing of the Stereotyped Behaviors symptoms, $r=-.41, p=.020$. The increasing of the Academic outcome was significantly related to the decreasing of the Stereotyped Behaviors symptoms, $r=-.49, p=.005$. The increasing of the School Community outcome was significantly related to the decreasing of the Stereotyped Behaviors symptoms, $r=-.43, p=$ .012. The increasing of the Daily Living Skills outcome was significantly related to the decreasing of the Stereotyped Behaviors symptoms, $r=-.48, p=.005$. The increasing of the 
Coping outcome was significantly related to the decreasing of the Stereotyped Behaviors symptoms, $r=-.48, p=.005$. The increasing of the Fine Motor outcome was significantly related to the decreasing of the Stereotyped Behaviors symptoms, $r=-.67, p=.034$. And finally the increasing of the Motor Skills outcome was significantly related to the decreasing of the Stereotyped Behaviors symptoms, $r=-.72, p=.020$.

Table 5.3

Dynamics between the Autism Symptoms and Outcomes at The Vista School

\begin{tabular}{|c|c|c|c|c|c|}
\hline & & Autism Index & $\begin{array}{r}\text { Stereotyped } \\
\text { Behaviors }\end{array}$ & Communication & $\begin{array}{r}\text { Social } \\
\text { Interaction }\end{array}$ \\
\hline $\begin{array}{l}\text { Adaptive } \\
\text { Behavior }\end{array}$ & Pearson & -.288 & $-.505^{* *}$ & -.075 & -.100 \\
\hline $\begin{array}{l}\text { Receptive } \\
\text { Language }\end{array}$ & Pearson & $-.418^{*}$ & $-.519^{* *}$ & -.124 & -.263 \\
\hline $\begin{array}{l}\text { Expressive } \\
\text { Language }\end{array}$ & Pearson & -.260 & $-.521^{* *}$ & -.114 & -.086 \\
\hline $\begin{array}{l}\text { Written } \\
\text { Language }\end{array}$ & Pearson & -.091 & $-.402^{*}$ & .084 & .132 \\
\hline $\begin{array}{l}\text { Communication } \\
\text { Domain }\end{array}$ & Pearson & -.226 & $-.491^{* *}$ & -.003 & -.017 \\
\hline Personal & Pearson & -.211 & -211 & -.185 & -.97 \\
\hline Domestic & Pearson & .243 & -.065 & .181 & .262 \\
\hline Academic & Pearson & -.287 & $-.481^{* *}$ & -.216 & -.079 \\
\hline Community & Pearson & -130 & -.319 & .042 & .118 \\
\hline $\begin{array}{l}\text { School } \\
\text { Community }\end{array}$ & Pearson & -.330 & $-.433^{*}$ & -.027 & -.263 \\
\hline $\begin{array}{l}\text { Daily Living } \\
\text { Skills }\end{array}$ & Pearson & -315 & $-.473^{* *}$ & -.205 & -.135 \\
\hline Interpersonal & Pearson & -.162 & -.150 & -.164 & -.095 \\
\hline Play and & Pearson & -.163 & -.075 & .045 & -.253 \\
\hline Leisure & (2-tailed) & .366 & .680 & .849 & .155 \\
\hline Coping & Pearson & -.318 & $-.478^{* *}$ & -.166 & -.081 \\
\hline Socialization & Pearson & -.278 & -.328 & -.106 & -.166 \\
\hline Gross Motor & Pearson & -.276 & -.580 & -.012 & .020 \\
\hline Fine Motor & Pearson & -.039 & $-.670^{*}$ & .250 & .384 \\
\hline Motor Skills & Pearson & -.164 & $-.717^{*}$ & .211 & .258 \\
\hline
\end{tabular}




\section{Autism Specialized Demographics}

Education. From the parents' survey responses $(\mathrm{N}=36)$, there were 34 families that have one child at The Vista School and 2 families that have two children at The Vista School. $97 \%$ of these children were males and only $3 \%$ were females. $18 \%$ of The Vista School students had attended the school for less than one year, $9 \%$ two years, $6 \%$ three years, $0 \%$ four years, $6 \%$ five years, $3 \%$ six years, $18 \%$ seven years, $3 \%$ eight years, $6 \%$ nine years, $6 \%$ ten years, $6 \%$ eleven years, and $21 \%$ twelve years. Regarding the parents' and/or legal guardians' education , $14 \%$ parents had earned a high school diploma, $47 \%$ earned a Bachelor of Art degree, $11 \%$ had a Master of Art degree, $11 \%$ had a Master of Science degree, $3 \%$ had earned a Medical Doctor degree, and $14 \%$ had experienced some other type of education. For the specific major, see Table 5.4 below

Table 5.4

Autism Demographics: Parents Education-Major at The Vista School

\begin{tabular}{|c|c|c|}
\hline \multicolumn{3}{|c|}{ Majors } \\
\hline English & Business & Criminal Justice \\
\hline Communications & Accounting & Communications \\
\hline Journalism/PR & & \\
\hline Psychology & Business Administration & Physical education \\
\hline College Prep & Political Science & Communications \\
\hline Education & Public Administration & $\begin{array}{l}\text { Electrical Engineering } \\
\text { Technology }\end{array}$ \\
\hline Elementary Education & Biblical Studies & School counseling \\
\hline Social Work & Law & Nursing \\
\hline Teaching and curriculum & Special Education & Telecommunication \\
\hline $\begin{array}{l}\text { Teaching elementary } \\
\text { education }\end{array}$ & Broadcast Journalism & Social Work \\
\hline
\end{tabular}


Medical. Regarding the medical-oriented demographics, 56\% of The Vista School students were first diagnosed at age 2 years old, $26 \%$ were first diagnosed at age 3,12\% were first diagnosed at age 4,3\% were first diagnosed at age 7, and 3\% were first diagnosed at age 12 . In addition to or previous to the diagnosis of Autism Spectrum Disorder, 26\% of students of The Vista School were diagnosed with Language Disorder, 29\% with Cognitive Delay, 12\% with Anxiety Disorder, 9\% with Mood Disorder, 0\% with Depression Disorder, 26\% with Attention Deficit Hyperactive Disorder, 0\% with Neurological Disorder, 6\% with Learning Disorder, $15 \%$ with some type of medical issue, $24 \%$ with Gastrointestinal Disorders, $9 \%$ with genetic issues, 9\% with Tic Disorder, 0\% with Fragile X Syndrome, 18\% with Epilepsy, 0\% Mendelian, 3\% with Bipolar Disorder, 26\% with other medical issues (Apraxia , PDD, Immune Deficiency, Intellectual Disability, Cancer, Tricatylamania, Smith-Magenis Syndrome, Diabetes $\&$ Perthes Disease). 26\% were diagnosed only with Autism Spectrum Disorder.

Concerning medication, $16 \%$ of The Vista School students take Luvox $^{\mathrm{TM}}, 21 \%$ take Zoloft $^{\mathrm{TM}}, 0 \%$ take Anafranil ${ }^{\mathrm{TM}}, 0 \%$ take Haloperidol, $5 \%$ take Chlorpromazine, $5 \%$ take Thioridazine, 0\% take Fluphenazine, 32\% take Risperidone, 0\% take Zyprexa, 11\% take Geodon, $21 \%$ take Clonidine, $11 \%$ take Tegretol, $0 \%$ take Lamictal, $0 \%$ take Topamax, $11 \%$ take Depakote, $0 \%$ take Ritalin, and 47\% take other (Citalopram, Buspar, Keppra, bilify (aripiprazole), exapro, Lexapro, Seroquel, Intuniv, and Adderall).

Other treatments for autism. Sixty five percent of The Vista School students received other treatment(s) for Autism and 35\% did not receive any other treatment but the Competent Learner Model at The Vista School. Specifically, 45\% received Discrete Trials Training, 45\% received Verbal Behavior training, 10\% School-Based Inclusion Model training, 10\% received Center for Autism and Related Disorders (CARD) training, 20\% Floortime training, 10\% 
received the Pyramid Approach to Education, 30\% received Sensory Integration training, 55\% Music Therapy, 5\% Vision Therapy, 50\% Gluten Free Diet, 35\% received Casein Free Diet, 5\% received Anti-Yeast Therapy, 30\% received Vitamin Therapy, and 25\% received other type of treatment (speech therapy, Tomatis Sound Therapy, at home program behavior).

Nine percent of the parents strongly agreed that their child benefited from the previous program before they were exposed to CLM, $16 \%$ agreed, $13 \%$ somewhat agreed, $16 \%$ were neutral, $6 \%$ somewhat disagreed, $16 \%$ disagreed, and $25 \%$ strongly disagreed $(\mathrm{M}=4.41, \mathrm{SD}=$ 2.12).

\section{Results from DEM Dimension 3: Environment}

\section{Parenting Stress Level}

The parenting stress was assessed to (a) measure the stress level of parents with children with Autism when their child start at The Vista School and (b) uncover whether or not the parents' stress statistically significantly decreased when their children had been students at The Vista School for two years (see results dimension 4:outcomes).

(A) The Degree of Parents Stress Level. The Parenting Stress Index-Short Form was administered to The Vista School parents. I interpreted the results according to the Abidin Interpretation Form, which interprets the results according to the follow parameters:

$>16-80$ Percentile $=$ Normal Range

> $81-84$ Percentile $=$ Borderline

$85-99^{+}$Percentile $=$Clinically Significant

$>$ Defensive Responding score less than 10 is clinically significant

I calculated the percentile position of each raw score to determine parents' level of stress and identified the percentages of the parents in each category. 


\section{Defensive Responding}

Table 5.5 below illustrates the frequency distribution and percentile of parental defensive responding. Five parents $(10 \%)$ responded below 10, indicating the tendency to minimize the stress in a parent-child relationship, consequently raising possible quest ions regarding the validity of the results, but $90 \%$ of the parents responded within the valid protocol range.

\section{Parental Distress}

Table 5.5 below illustrates the frequency distribution and percentile of Parental Distress. Fifty-five percent of the parents are within a normal range, indicating that these parents are experiencing a normal degree of stress associated with their role and adjustment as a parent to a child with Autism. Seven percent of the parents have borderline stress levels, and thirty-eight percent of The Vista School parents are clinically significantly distressed in their role as parent of a child with Autism.

\section{Parent-Child Dysfunctional Interaction}

Twenty-three percent of the parents are within the normal range, indicating satisfactory expectations and parent-child interactions. However, seventy-seven percent of the parents scored within the clinically significant range, indicating they are highly distressed parents by the quality of parent-child interactions. Possibly the child does not meet the parental expectations, or the parent may feel alienated and rejected by her/his child's Autistic behavior. See Table 5.5 below for the frequency distribution and percentile of the Parent-Child Dysfunction Interaction.

\section{Difficult Child}

Eighteen percent of the parents are within the normal range indicating satisfactory parentchild relationships because the parent perceives child characteristics as normal. Eighty-two percent of the parents scored within the clinically significant range, indicating that child behavior 
is markedly upsetting or destructive to the relationship between the parent and a child with

Autism. See Table 5.5 below for the frequency distribution and percentile of the Difficult Child.

\section{Total Parental Stress}

Twenty percent of the parents are within the normal range, indicating that the parent has no more stress than the average parent. Eighty percent of the parents scored within the clinically significant range indicating highly stressed out parents when parenting their child with Autism.

See Table 5.5 below for the frequency distribution and percentile of the Total Parental Stress.

Table 5.5

Parenting Stress Index at The Vista School

\begin{tabular}{|c|c|c|c|c|c|c|}
\hline \multirow[t]{2}{*}{ Subscale } & \multirow[t]{2}{*}{ Mean } & \multirow[t]{2}{*}{ SD } & \multirow[t]{2}{*}{$\mathrm{N}$} & \multicolumn{3}{|c|}{ Category \% } \\
\hline & & & & $\begin{array}{l}\text { Normal } \\
\text { Range }\end{array}$ & Borderline & $\begin{array}{c}\text { Clinically } \\
\text { Significant }\end{array}$ \\
\hline Defensive Responding & 18.39 & 6.22 & 49 & $90 \%$ & - & $10 \%$ \\
\hline Parental Distress & 32.11 & 8.91 & 45 & $55 \%$ & $7 \%$ & $38 \%$ \\
\hline $\begin{array}{l}\text { Parent Child } \\
\text { Dysfunctional Interaction }\end{array}$ & 29.27 & 5.85 & 44 & $23 \%$ & - & $77 \%$ \\
\hline $\begin{array}{l}\text { Difficult Child } \\
\text { lat }\end{array}$ & 40.34 & 9.23 & 44 & $18 \%$ & - & $82 \%$ \\
\hline Total Stress & 102.09 & 19.09 & 44 & $25 \%$ & - & $80 \%$ \\
\hline
\end{tabular}

\section{Satisfaction with Ultimate Outcomes of the CLM}

\section{Parents' and/or caregivers' satisfaction with the Ultimate Outcomes of the}

CLM. The In average parents and/or caregivers (N=37) identified the highest amount of satisfaction with their child's development as a problem solver $(\mathrm{M}=6.03, \mathrm{SD}=0.88)$. Parents agree that the repertoires of participator $(M=6.00, S D=0.94)$, listener $(M=5.79, S D=1.29)$, and observer $(\mathrm{M}=5.70, \mathrm{SD}=1.21)$ have improved. The least amount of satisfaction was with the writer repertoire $(\mathrm{M}=4.63, \mathrm{SD}=1.54)$. In sum, parents and/or caregivers are most satisfied with development of the problem solver behavioral repertoire and least satisfied with 
development of the writer behavioral repertoire. The reliability statistics on this scale indicated a high level of internal consistency Cronbach's $\alpha=.86$. The items were evaluated based on the 7 level Likert scale where $1=$ Strongly Agree, $2=$ Agree, $3=$ Somewhat Agree, $4=$ Neutral, $5=$ Somewhat Disagree, $6=$ Disagree, and $7=$ Strongly Disagree. The items in table 5.6 are ordered from highest to lowest agreement.

Table 5.6

Likert-type Survey Item: Parents and/or caregivers satisfaction with the CLM Ultimate Outcomes

\begin{tabular}{clcc}
\hline $\begin{array}{c}\text { Item } \\
\#\end{array}$ & Likert-type Survey Items & Mean & \\
\hline PSR & I believe that overall my child's solving skills have improved & 6.03 & 0.88 \\
PR & I believe that overall my child's participates more in learning & 6.00 & 0.94 \\
LR & I believe that overall my child's listening skills have improved & 5.79 & 1.29 \\
OR & I believe that overall my child's observing skills have improved & 5.70 & 1.21 \\
TR & I believe that overall my child's talking skills have improved & 5.24 & 1.75 \\
RR & I believe that overall my child's reading skills have improved & 5.21 & 1.56 \\
WR & I believe that overall my child's writing skills (have improved & 4.63 & 1.54 \\
\hline
\end{tabular}

Parents' and/or caregivers' satisfaction with The Vista School. Parents are very satisfied with the school $(\mathrm{M}=6.70, \mathrm{SD}=0.92)$ and would recommend The Vista School to other parents of children with Autism $(\mathrm{M}=6.67, \mathrm{SD}=0.92)$. In addition, parents agree that The Vista School employs rigorous and systematic teaching $(\mathrm{M}=6.51, \mathrm{SD}=0.97)$. The reliability statistics on this scale indicated excellent internal consistency Cronbach's $\alpha=.96$. The items were evaluated based on the 7 level Likert scale where $7=$ Strongly Agree, $6=$ Agree, $5=$ Somewhat 
Agree, 4 = Neutral, 3 = Somewhat Disagree, $2=$ Disagree, and $1=$ Strongly Disagree. The items in table 5.7 below are ordered from highest to lowest agreement.

Table 5.7

Likert-type Survey Item: Parents' Satisfaction with The Vista School

\begin{tabular}{|c|c|c|c|}
\hline Item \# & Likert-type Survey Item & Mean & SD \\
\hline PV3 & $\begin{array}{l}\text { I recommend The Vista School for other children with } \\
\text { Autism }\end{array}$ & 6.70 & 0.92 \\
\hline PV2 & Overall, how satisfied are you with The Vista School? & 6.67 & 0.92 \\
\hline PV1 & $\begin{array}{l}\text { I feel that The Vista School employs rigorous and } \\
\text { systematic teaching }\end{array}$ & 6.51 & 0.97 \\
\hline
\end{tabular}

Parents and/or caregivers responded to two open-ended questions about The Vista School (see table A3/3, appendix3 for a complete transcription of all answers). I asked them: (1) what do you think is the strongest component of The Vista School and (2) please help us to understand in what ways The Vista School can be improved. What would you like to change? For both questions, I employed the Thematic Analysis for the textual responses to open-ended survey questions. Therefore, I identified and coded the main themes to uncover the parents' experiences with their children's involvement in The Vista School and the CLM curriculum. I identified the four main clusters by clear repetition of patterns in each question. The four clusters emerged from the inductive analytical process of detecting the similarities in the textual answers. To be able to see the clusters, I de-contextualized the data by separating each answer, and put them into a table organization to be able to systematically search for emerging themes. Subsequently, I recontextualized the textual data by creating and analyzing the themes. 


\section{Q1: What do you think is the strongest component of The Vista School?}

I identified four main clusters uncovering what are the strongest components of The Vista School. Clusters are organized from the most prevalent to least dominant.

1. $\operatorname{Staff}(\mathrm{N}=18)$

2. Satisfaction with The Vista School $(\mathrm{N}=7)$

3. Behavioral and academic improvement $(\mathrm{N}=6)$

4. CLM systematic approach $(\mathrm{N}=6)$

See table 5.8 below for an example of the answers sorted by the clusters.

Table 5.8

Sample of Parent's Identification of the Strongest Components of The Vista School

\begin{tabular}{|c|c|}
\hline Cluster & Abbreviated Responses \\
\hline Staff & $\begin{array}{l}\text { Staff is amazing; staff and how they work; staff makes school } \\
\text { successful; staff dedication, staff love of our children, without staff } \\
\text { Vista would be just another school; staff are awesome, staff who truly } \\
\text { care, staff commitment, caring staff, wonderful staff, staff are by far } \\
\text { the best I have seen; staff who work directly with my son; staff makes } \\
\text { a priority to include family. }\end{array}$ \\
\hline $\begin{array}{l}\text { Satisfaction with The } \\
\text { Vista School }\end{array}$ & $\begin{array}{l}\text { The Vista School address my son behavior, I recommend The Vista } \\
\text { School to anybody, Vista is blessing, I love the school in so many } \\
\text { ways; I am pleased with Vista; I am 100\% pleased with Vista and the } \\
\text { CLM model. }\end{array}$ \\
\hline $\begin{array}{l}\text { Behavioral and } \\
\text { Academic } \\
\text { improvement }\end{array}$ & $\begin{array}{l}\text { My son now ask for help; he is making tremendous progress, he } \\
\text { speaks sentences most of the time; my son improved so much } \\
\text { behaviorally and academically; he understands how to use toiled. }\end{array}$ \\
\hline $\begin{array}{l}\text { CLM Systematic } \\
\text { approach }\end{array}$ & $\begin{array}{l}\text { The latest teaching methods; ABA; work systematically to achieve } \\
\text { the goal; teaching is great, commitment to the model; comprehensive } \\
\text { and systematic approach to behavioral management. }\end{array}$ \\
\hline
\end{tabular}




\section{Q2: Please help us to understand in what ways The Vista School can be improved.}

\section{What would you like to change?}

I identified three main clusters uncovering in what ways The Vista School can be improved and what the parents would like to change. Clusters are organized from the most prevalent to least dominant.

1. Adult Program $(\mathrm{N}=14)$

2. Communication $(\mathrm{N}=7)$

3. Other changes and/or improvement $(\mathrm{N}=12)$

Table 5.9

Sample of Parents' Suggestions for improvement and/or changes at The Vista School

\begin{tabular}{ll}
\hline \multicolumn{1}{c}{ Cluster } & \multicolumn{1}{c}{ Abbreviated Responses } \\
\hline Adult Program & $\begin{array}{l}\text { Get the adult program up; create an adult program; provide } \\
\text { quality care for adults; work living program; adult services; over } \\
21 \text { program; residential care. }\end{array}$ \\
Communication & $\begin{array}{l}\text { Communication with other parents; stronger communication } \\
\text { between parents and teachers; better communication with the } \\
\text { personal. }\end{array}$ \\
Other & $\begin{array}{l}\text { Longer school days; workshop for parents; add a day program; } \\
\text { Dr. note hand in; music therapist; expend after school services; } \\
\text { bigger enrolment; pre-school programming; more use of } \\
\text { technology in teaching; homework assignments; literacy based } \\
\text { program; involvement in sport, Special Olympics. }\end{array}$ \\
\hline
\end{tabular}

Administration and staff satisfaction with the Ultimate Outcomes of the CLM. The

Vista School has students with large variability and different degrees of Autism symptoms and/or comorbid developmental or learning disabilities. Therefore the items investigating satisfaction with the ultimate outcomes of the CLM were organized on (a) improvement in the 
behavioral repertoires for SOME students and (b) improvement in the behavioral repertoires for ALL students.

(A) Teachers and/or other staff of The Vista School identified (see table 5.10 below) that the CLM is most successful in improvement of the ultimate outcomes in SOME of their students, particularly in development of the participator $(\mathrm{M}=5.65, \mathrm{SD}=1.08)$, observer $(\mathrm{M}=$ $5.61, \mathrm{SD}=0.97)$ and problem solver $(\mathrm{M}=5.60, \mathrm{SD}=1.03)$. The least successful improvement in some of the students as a result of the CLM implementation at The Vista School is reader $(\mathrm{M}=$ 4.80, $\mathrm{SD}=1.30)$, writer $(\mathrm{M}=4.90, \mathrm{SD}=1.23)$ and listener $(\mathrm{M}=5.40, \mathrm{SD}=1.02)$. The reliability statistics on this scale indicated a high level of internal consistency Cronbach's $\alpha=$ .82. The items were evaluated based on the 7 level Likert scale where $7=$ Strongly Agree, $6=$ Agree, $5=$ Somewhat Agree, $4=$ Neutral, $3=$ Somewhat Disagree, $2=$ Disagree, and $1=$ Strongly Disagree. The items in the table 5.10 below are ordered from highest to lowest agreement.

Table 5.10

Likert-type Survey Item: Teachers' assessment of the CLM Ultimate Outcomes for SOME Students

\begin{tabular}{clrc}
$\begin{array}{c}\text { Item } \\
\#\end{array}$ & \multicolumn{1}{c}{ Likert-type Survey Item } & Mean & SD \\
\hline T15 & I believe that SOME of my students overall participate more in learning & 5.65 & 1.08 \\
T9 & I believe that SOME of my students' overall observing skills have improved & 5.61 & 0.97 \\
T5 & I believe that SOME of my students' overall problem solving skills have & 5.60 & 1.03 \\
& improved & 5.40 & 1.02 \\
T13 & I believe that SOME of my students' overall listening skills have improved & 5.25 & 1.04 \\
T11 & I believe that SOME of my students' overall talking skills have improved & 4.90 & 1.23 \\
T3 & I believe that SOME of my students' overall writing skills have improved & 4.80 & 1.30 \\
T7 & I believe that SOME of my students' overall reading skills have improved & &
\end{tabular}


(B) Regarding improvement in the behavioral repertoires for ALL students (see table 5.11

below), teachers, administration and/or other staff of The Vista School identified that the CLM in their view is most successful in improvement of the ultimate outcomes of the problem solver (M $=5.00, \mathrm{SD}=1.41)$, participator $(\mathrm{M}=4.96, \mathrm{SD}=1.38)$ and observer $(\mathrm{M}=4.76, \mathrm{SD}=1.62)$. The least successful improvement in ALL of the students as a result of the CLM implementation at The Vista School is Writer $(\mathrm{M}=3.32, \mathrm{SD}=1.46)$, reader $(\mathrm{M}=3.931, \mathrm{SD}=1.60)$ and talker $(\mathrm{M}$ $=3.94, \mathrm{SD}=1.57)$. The reliability statistics on this scale indicated a high level of internal consistency Cronbach's $\alpha=.84$.

Table 5.11

Likert-type Survey Item: Teachers' assessment of the CLM Ultimate Outcomes for ALL Students Item Likert-type Survey Item Mean SD \#

T4 I believe that ALL of my students' overall problem solving skills have $\quad \begin{array}{lll}5.00 & 1.41\end{array}$ improved

T14 I believe that overall ALL of my students participate more in learning $\quad 4.96 \quad 1.38$

T8 I believe that ALL of my students' overall observing skills have improved $4.76 \quad 1.62$

T12 I believe that ALL of my overall students' listening skills have improved $\quad 4.70 \quad 1.49$

T10 I believe that ALL of my students' overall talking skills have improved $\quad 3.94 \quad 1.57$

T6 I believe that ALL of my students' overall reading skills have improved $\quad 3.93 \quad 1.60$

T2 I believe that ALL of my students' overall writing skills improved $\quad 3.32 \quad 1.46$

Teachers', administration and/or staff satisfaction with The Vista School. Teachers, administration and staff members somewhat agree that The Vista School employs rigorous and systematic teaching $(\mathrm{M}=5.43, \mathrm{SD}=1.37)$. 


\section{Teachers', administration and/or staff satisfaction with other components of the CLM.}

The teachers and/or staff feel most comfortable obtaining assistance from a BCBA $(\mathrm{M}=5.83$, $\mathrm{SD}=1.8)$, using CLM Curriculum Instructional Formats $(\mathrm{M}=5.61, \mathrm{SD}=1.13)$, and using CLM support material in the classroom $(\mathrm{M}=5.55, \mathrm{SD}=1.31)$. Teachers and staff members are least satisfied with the preparation by a coach to use the CLM components $(M=4.74, S D=1.71)$, and with the Component Learner Model Repertoire Assessment $(\mathrm{M}=4.74, \mathrm{SD}=1.27)$. The reliability statistics on this scale indicated excellent internal consistency Cronbach's $\alpha=.94$. The items were evaluated based on the 7 level Likert scale where $7=$ Strongly Agree, $6=$ Agree, $5=$ Somewhat Agree, 4 = Neutral, $3=$ Somewhat Disagree, $2=$ Disagree, and $1=$ Strongly Disagree . The items in the table 5.12 below are ordered from highest to lowest agreement. 
Table 5.12

Likert-type Survey Item: Teachers, administration and staff satisfaction with other components of CLM

\begin{tabular}{clcc}
\hline $\begin{array}{c}\text { Item } \\
\#\end{array}$ & Likert-type Survey Item & Mean & SD \\
\hline Q18 & $\begin{array}{l}\text { I feel that I can obtain assistance from a BCBA with difficult student behavior } \\
\text { in a timely manner }\end{array}$ & 5.83 & 1.18 \\
Q35 & I feel comfortable using the CLM Curriculum Instructional Formats & 5.61 & 1.13 \\
Q36 & I am using CLM support materials in my classroom & 5.55 & 1.31 \\
Q9 & Overall, how satisfied are you with the CLM Curriculum? & 5.16 & 1.30 \\
Q17 & I feel that I can obtain assistance from my CLM coach with difficult student & 5.13 & 1.70 \\
& behavior in a timely manner & 5.00 & 1.23 \\
Q10 & Overall, how satisfied are you with the entire CLM? & 4.94 & 1.36 \\
Q11 & The CLM Course of Study materials are easy to use & 4.90 & 1.14 \\
Q5 & Overall, how satisfied are you with the CLM Collaborative Consultation? & 4.86 & 1.18 \\
Q13 & The CLM Curriculum materials are easy to use & 4.84 & 1.59 \\
Q7 & Overall, how satisfied are you with the CLM Coaching? & 4.84 & 1.23 \\
Q14 & Overall the CLM materials are easy to use & 4.77 & 1.20 \\
Q8 & Overall, how satisfied are you with the Competent Learner Repertoire & & \\
& Assessments? & 4.74 & 1.27 \\
Q12 & The Competent Learner Model Repertoire Assessments (CLRA) are easy to & & \\
& use & 4.74 & 1.71 \\
Q16 & I feel well supported by my coach when I teach students using the components \\
& of the CLM & 4.71 & 1.32 \\
Q6 & Overall, how satisfied are you with the CLM Course of Study? & 4.22 & 1.63 \\
Q15 & I feel I was well prepared by my coach to use the components of the CLM & & \\
\hline
\end{tabular}

Administration, teachers, and/or staff also responded to two open-ended questions about The Competent Learner Model (see table A3/4, appendix 3 for complete transcription of all answers). I asked the following questions: (1) what do you think is the strongest component of the CLM and (2) please help us to understand in what ways the CLM can be improved. What would you like to change? For both questions, I employed Thematic Analysis for the textual responses to the open-ended survey question. Therefore, I identified and coded the main themes to uncover the teachers', administration and staff experiences with the Competent Learner Model. I identified the seven main clusters by clear repetition of patterns in each question. The 
seven clusters emerged from the inductive analytical process of detecting the similarities in the textual answers. To be able to see the clusters, I de-contextualized the data by separating each answer, and put them into a table organization to be able to systematically search for emerging themes. Subsequently, I re-contextualized the textual data by creating and analyzing the themes.

\section{Q1: What do you think is the strongest component of the CLM?}

I identified seven main clusters uncovering what are the strongest components of the Competent Learner Model. Clusters are organized from the most prevalent to least dominant.

1. Repertoires $(\mathrm{N}=6)$

2. Consistency $(\mathrm{N}=5)$

3. Coaching $(\mathrm{N}=4)$

4. Problem Solver $(\mathrm{N}=3)$

5. Early Lessons $(\mathrm{N}=3)$

6. Flexibility toward Learner $(\mathrm{N}=3)$

7. Other $(\mathrm{N}=8)$ 
Table 5.13

Sample of Teachers and Staff identification of the strongest components of the CLM

\begin{tabular}{ll}
\hline \multicolumn{1}{c}{ Cluster } & \multicolumn{1}{c}{ Abbreviated Responses } \\
\hline Repertories & $\begin{array}{l}\text { Repertoires; Identifying and outlining the } 7 \text { repertoires; how all } \\
\text { repertoires work together; understanding of the repertoires; } \\
\text { repertoires }\end{array}$ \\
Consistency & $\begin{array}{l}\text { Repetition in teaching the material; Consistency in teaching; } \\
\text { formats that create consistency; systematic }\end{array}$ \\
Coaching & Coaching system; the coaching; coaching support assistance \\
Problem Solver & $\begin{array}{l}\text { Problem solver; problem solver } \\
\text { Early Lessons }\end{array}$ \\
Flexibility toward Learner & $\begin{array}{l}\text { Curriculum adapts for students; look at learners strengths and } \\
\text { use them; }\end{array}$ \\
Other & $\begin{array}{l}\text { Visual poster boards; lessons break down; collaborative } \\
\text { consultations; behavioral theory; the formats; the scope and } \\
\text { sequence }\end{array}$ \\
\hline
\end{tabular}

\section{Q2: In what ways the CLM can be improved}

I identified seven main clusters uncovering in what ways the Competent Learner Model can be improved. Clusters are organized from the most prevalent to least dominant.

1. Coaching Time $(\mathrm{N}=9)$

2. Use Friendly Materials $(\mathrm{N}=7)$

3. Checkout $(\mathrm{N}=4)$

4. Programming $(\mathrm{N}=4)$

5. Course of Study $(\mathrm{N}=3)$

6. Video $(\mathrm{N}=3)$

7. Other $(\mathrm{N}=7)$ 
Table 5.14

Sample of Teachers' and Staff suggestions for improvement of the CLM

\begin{tabular}{ll}
\hline \multicolumn{1}{c}{ Cluster } & \multicolumn{1}{c}{ Abbreviated Responses } \\
\hline Couching Time & Have direct time with coach; get individualized time with \\
coach; amount of time spent with coaches; increase number of \\
coaches; additional coaches; support from classroom coaches; \\
available coach, meeting with coach within a few days after \\
completion of units and application, we need to be trained with \\
fidelity, we don't have enough "in the moment" training in the \\
classroom, have trainers in the classroom on daily basis
\end{tabular}




\section{Results from DEM Dimension 4: Outcomes}

\section{Adaptive Behavior}

The analyses indicated (see table 5.15 below) statistically significantly results in developing four measured adaptive behavior subscales. Specifically, on the average, The Vista School students' adaptive behavior statistically significantly improved from before the CLM implementation until two years later. It improved in the School Community subscale from the initial measurement $(\mathrm{M}=6.77, \mathrm{SE}=.28)$ to two years later $(\mathrm{M}=7.39, \mathrm{SE}=.16), t(74)=2.67, p$ $=.011$, which represented a medium sized effect, $d=0.32$. It statistically significantly improved in the Play and Leisure subscale from the initial measurement $(\mathrm{M}=6.65, \mathrm{SE}=.16)$ to two years later $(\mathrm{M}=7.40, \mathrm{SE}=.16), t(74)=-5.16, p=.000$, which represented a medium sized effect, $d=$ 0.56. It statistically significantly improved in the Coping subscale from the initial measurement $(\mathrm{M}=7.19, \mathrm{SE}=.19)$ to two years later $(\mathrm{M}=7.83, \mathrm{SE}=.19), t(74)=-3.47, p=.001$, which represented a medium sized effect, $d=0.40$. And it statistically significantly improved in the Socialization subscale from the initial measurement $(\mathrm{M}=54.20, \mathrm{SE}=.77)$ to two years later $(\mathrm{M}$ $=57.04, \mathrm{SE}=.87), t(74)=-4.01, p=.000$, which represented a medium sized effect, $d=0.31$.

The Competent Learned Model was statistically significantly not successful in three measured adaptive behavior subscales. Explicitly, on the average, the adaptive behavior of Vista School students statistically significantly declined from before the CLM implementation until two years later in the subscales of Written Language, with an initial measurement of $(\mathrm{M}=7.68$, $\mathrm{SE}=.40)$ to two years later $(\mathrm{M}=7.23, \mathrm{SE}=.34), t(74)=2.13, p=.037$, which represented a small sized effect, $d=0.13$. It statistically significantly declined in Academic, from the initial measurement of $(\mathrm{M}=6.71, \mathrm{SE}=.39)$ to two years later $(\mathrm{M}=6.27, \mathrm{SE}=.34), t(74)=2.13, p=$ .036 , which represented a small sized effect, $d=0.14$. And it statistically significantly declined 
in Community, from the initial measurement of $(\mathrm{M}=6.22, \mathrm{SE}=.36)$ to two years later $(\mathrm{M}=$ $5.41, \mathrm{SE}=.39), t(48)=4.05, p<.000$, which represented a medium sized effect, $d=0.31$.

The Competent Learner Model did not produce any statistically significant changes in the follow adaptive behavior domains: Adaptive Behavior, Receptive Language, Expressive Language, Communication, Personal, Domestic, Daily Living Skills, Interpersonal, Gross Motor Skills. Fine Motor Skills and Motor Skills. For Motor Skills , a non-parametric Wilcoxon signedrank test comparison was done, given not meeting assumption of normality (see chapter 5). There was no statistically significant result in Motor Skill from the initial measurement $(M d n=$ 61.00) to two years later $(M d n=59.50), T=160, p=.213$.

Table 5.15

Pair Sample Test for The Vineland Adaptive Behavior Scales

\begin{tabular}{|c|c|c|c|c|c|c|c|c|}
\hline Pair & Subscale & Mean & SD & $\begin{array}{l}\text { Std. } \\
\text { Error }\end{array}$ & $\mathrm{t}$ & df & $\begin{array}{c}\text { Sig. } \\
\text { (2-tailed) }\end{array}$ & $\begin{array}{l}\text { Effect } \\
\text { Size } d\end{array}$ \\
\hline Pair 1 & Adaptive Behavior & -1.32 & 6.87 & .79 & -1.67 & 74 & .100 & - \\
\hline Pair 2 & Receptive Language & -.16 & 1.54 & 18 & -.89 & 74 & 372 & - \\
\hline Pair 3 & $\begin{array}{l}\text { Expressive } \\
\text { Language }\end{array}$ & -.05 & 1.04 & .12 & -.45 & 74 & 658 & - \\
\hline Pair 4 & Written Language & .45 & 1.85 & .21 & 2.13 & 74 & $.037^{*}$ & 0.13 \\
\hline Pair 5 & Communication & .57 & 7.23 & .83 & .69 & 74 & 494 & - \\
\hline Pair 6 & Personal & -.44 & 2.10 & .24 & -1.81 & 74 & .074 & - \\
\hline Pair 7 & Domestic & .53 & 1.94 & .28 & 1.92 & 48 & .061 & - \\
\hline Pair 8 & Academic & .44 & 1.79 & .21 & 2.13 & 74 & $.036^{*}$ & 0.14 \\
\hline Pair 9 & Community & .81 & 1.41 & .20 & 4.05 & 48 & $.000^{*}$ & 0.31 \\
\hline Pair 10 & School Community & -.61 & 2.03 & .24 & -2.61 & 74 & $.011^{*}$ & 0.32 \\
\hline Pair 11 & Daily Living Skills & -1.48 & 8.25 & .95 & -1.55 & 74 & .125 & - \\
\hline Pair 12 & Interpersonal & -.25 & 1.51 & .174 & -1.46 & 74 & .150 & - \\
\hline Pair 13 & Play and Leisure & -.75 & 1.25 & .15 & -5.16 & 74 & $.000^{*}$ & 0.56 \\
\hline Pair 14 & Coping & -.64 & 1.59 & .19 & -3.5 & 74 & $.001^{*}$ & 0.40 \\
\hline Pair 15 & Socialization & -2.84 & 6.13 & .71 & -4.01 & 74 & $.000^{*}$ & 0.31 \\
\hline Pair 16 & Gross Motor Skills & .39 & 2.79 & .40 & .97 & 48 & .337 & - \\
\hline Pair 17 & Fine Motor Skills & -.25 & 2.22 & .32 & -.77 & 48 & .444 & - \\
\hline
\end{tabular}




\section{Autistic Symptoms}

The analyses indicated (see table 5.16 below) statistically significantly results in the development of two measured Autism symptoms subscales. Specifically, on the average, the Autism symptoms of students at The Vista School statistically significantly decreased from before the CLM implementation until two years later in the subscales of Social Interaction, from an initial measurement of $(\mathrm{M}=8.00, \mathrm{SE}=.59)$ to two years later $(\mathrm{M}=6.06, \mathrm{SE}=.32), t(32)=$ $3.65, p=.001$, which represented a large sized effect, $d=0.71$. Students statistically significantly improved (manifested less Autism) in the subscale of the Autism Index. For the Autism Index, a non-parametric Wilcoxon signed-rank test comparison was done given not meeting the assumption of normality (see chapter 5). There was a statistically significant result in the Autism Index from the initial measurement $(M d n=94.00)$ to two years later $(M d n=82.00), T=92.50, p$ $=.004$, which represented a large sized effect $d=0.78$.

The Competent Learner Model did not produce any statistically significant changes in the subscales of Stereotyped Behaviors and Communications.

Table 5.16

Paired Sample Test for The Gilliam Autism Rating Scales for Autistic Symptoms

\begin{tabular}{clccccccc}
\hline Pair & \multicolumn{1}{c}{ Subscale } & Mean & SD & $\begin{array}{c}\text { Std. } \\
\text { Error }\end{array}$ & $\mathrm{t}$ & $\mathrm{df}$ & $\begin{array}{c}\text { Sig. } \\
(2-\text { tailed })\end{array}$ & $\begin{array}{c}\text { Effect } \\
\text { Size }\end{array}$ \\
\hline Pair 20 & Gilliam Stereotyped & .52 & 2.09 & .36 & 1.41 & 32 & .167 & - \\
Pair 21 & Gilliam Communication & .65 & 3.17 & .71 & .92 & 19 & .370 & - \\
Pair 22 & Gilliam Social & 1.94 & 3.05 & .53 & 3.65 & 32 & $.001^{*}$ & 0.71 \\
& Interaction & & & & & & & \\
\hline
\end{tabular}




\section{Language Development}

The analyses indicated (see table 5.17 below) statistically significantly results in developing both subscales of (1) Auditory Comprehension and (2) Expressive Communication. For Auditory Comprehension, a non-parametric Wilcoxon signed-rank test comparison was done given not meeting the assumption of normality (see chapter 5). There was a statistically significant result in the Auditory Comprehension from the initial measurement $(M d n=31.00)$ to two years later $(M d n=35.00), T=616.50, p=.000$, which represented a medium sized effect $d$ $=0.49$. Expressive Comprehension was statistically significantly improved from the initial measurement $(\mathrm{M}=32.26, \mathrm{SE}=1.87)$ to two years later $(\mathrm{M}=35.59 \mathrm{SE}=2.09), t(38)=-5.46, p$ $=.000$, which represented a small sized effect, $d=0.24$.

Table 5.17

Pair Sample Test for Auditory and Expressive Comprehension of The Preschool Language Rating Scales

\begin{tabular}{clccccccc}
\hline Pair & \multicolumn{1}{c}{ Subscale } & Mean & SD & $\begin{array}{c}\text { Std. } \\
\text { Error }\end{array}$ & t & df & $\begin{array}{c}\text { Sig. } \\
\text { (2 tailed) }\end{array}$ & $\begin{array}{c}\text { Effect } \\
\text { Size }\end{array}$ \\
\hline Pair 24 & $\begin{array}{l}\text { Expressive } \\
\text { Communication }\end{array}$ & -4.33 & 4.95 & .79 & -5.46 & 38 & $.000^{*}$ & 0.24 \\
\hline
\end{tabular}

\section{Degree of Parents' Stress}

The analyses indicated (see table 5.18 below) statistically significantly results developing in two subscales of the Difficult Child and Total Stress. For the Total Stress subscale, a nonparametric Wilcoxon signed-rank test comparison was given not meeting the assumption of normality (see chapter 5). There was a statistically significant decrease in Total Stress from the initial measure $(M d n=103.50)$ to two years later $(M d n=98.00), T=6.50, p=.011$, which represented a medium sized effect $d=0.64$. There was also a statistically significant result in the 
Difficult Child subscale, from the initial measurement $(\mathrm{M}=45.79, \mathrm{SE}=2.43)$ to two years later $(\mathrm{M}=40.29 \mathrm{SE}=2.47), t(13)=3.70, p=.003$, which represented a medium sized effect, $d=$ 0.56 .

The Competent Learner Model did not produce any statistically significant changes in the follow subscales: Defense Responding, Parental Distress, and Parent Child Dysfunctional Interactions.

Table 5.18

Paired Sample Test for The Parenting Stress Index

\begin{tabular}{clccccccc}
\hline Pair & \multicolumn{1}{c}{ Subscale } & Mean & SD & $\begin{array}{c}\text { Std. } \\
\text { Error }\end{array}$ & t & df & $\begin{array}{c}\text { Sig. } \\
\text { (2-tailed) }\end{array}$ & $\begin{array}{c}\text { Effect } \\
\text { Size }\end{array}$ \\
\hline Pair 25 & Defense Responding & 1.65 & 4.66 & 1.13 & 1.46 & 16 & .165 & - \\
Pair 26 & Parental Distress & 3.07 & 7.75 & 2.00 & 1.53 & 14 & .148 & - \\
& Parent Child & 2.29 & 4.87 & 1.30 & 1.76 & 13 & .103 & - \\
Pair 27 & Dysfunctional & & & & & & & \\
& Interactions & 5.50 & 5.56 & 1.49 & 3.70 & 13 & $.003^{*}$ & 0.56 \\
\hline
\end{tabular}

\section{Results from DEM Dimension 5: Understanding}

The objective of this dimension of evaluation was to bring understanding by integrating data from all four Dynamic Evaluation Model Dimensions (D1- D4). Specifically, I aimed to do the following:

(1) Determine whether or not the treatment effective

(2) Understand the context of treatment implementation

(3) Understand who benefits the most from what particular intervention

(4) Understand what programs are able to produce what outcomes 
(5) Understand the differences among treatments

(6) Develop a three dimensional matrix of intervention components by child symptoms characteristics and behavior outcomes

(7) Tabulate the intensity and frequency of Autism symptoms (their distribution)

(8) Understand the dynamics of Autism symptomology (the relationships among symptoms)

(9) Contribute to the explanation of the phenomenon of differential responsiveness to treatments

\section{Determine whether or not the treatment is effective}

The Competent Learner Model seems to be effective in developing the following adaptive behavior subscales in children with Autism:

$>$ School Community

$>$ Play and Leisure

$>$ Coping

Social Interaction (Especially Effective)

Autism Index (Especially Effective)

Expressive Communication

Auditory Comprehension

The Competent Learner Model seems to be effective in mitigating stress in parents of children with Autism in the follow subscales:

$>$ Difficult Child

Total Stress 
The Competent Learner Model seems not to be effective in developing the following adaptive behavior subscales in children with Autism:

Written Language

Academics

Community

The Competent Learner Model seems to be neutral in developing the following adaptive behavior subscales in children with Autism:

Adaptive Behavior

$>$ Receptive Language

Expressive Language

Communication

$>$ Personal

$>$ Domestic

$>$ Daily Living Skills

$>$ Interpersonal

$>$ Gross Motor Skills

Fine Motor Skills

\section{Context of the Implementation of the CLM}

\section{Identification of Who Benefits the Most from What Particular Intervention}

From this evaluation it emerged that the Competent Learner Model is a better match for children with a severe form of Autism (see figure 5.5 below). It is apparent that before the treatment (a) there is high variability in Autism Index scores among students and (b) the vast 
majority of the students scored above 90. After the CLM treatment, the variability was reduced and the vast majority of the students tended to cluster between scores of 70 and 90.
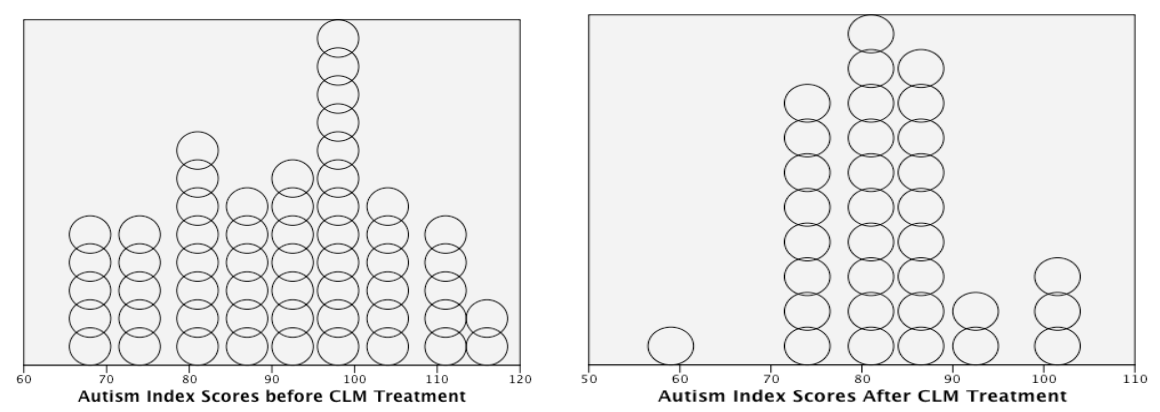

Figure 5.1 Autism Index Scores Before and After CLM Treatment. Figures illustrates the reduction in variability in Autism Index

The results from the correlation matrix mapping the dynamics between Autism

Symptoms and the Competent Learner Model Outcomes suggest that the Competent Learner Model significantly negatively correlates (the more adaptive behavior is developed, the less Autism is present) between:

Adaptive Behavior:

Receptive Language

Expressive Language

Written Language

Stereotyped Behavior

Communication

AND Characteristic Autism symptoms

Academic

School Community

Daily Living Skills

Coping

Fine Motor

Motor Skills

This would suggest that the Competent Learner Model might be a good fit for children with a predominant manifestation of stereotyped Autistic behavior. 


\section{Outcomes Produced by the CLM}

The Competent Learner Model produces statistically significant outcomes in: School Community, Play and Leisure, Coping, Social Interaction, Autism Index, Expressive Communication, and Auditory Comprehension.

The Competent Learner Model produced the following ultimate outcomes that were rated by parents and teachers in a similar sequence of success. (See table 5.19 below) The items were evaluated based on the 7 level Likert scale where $7=$ Strongly Agree, $6=$ Agree, $5=$ Somewhat Agree, $4=$ Neutral, $3=$ Somewhat Disagree, $2=$ Disagree, and $1=$ Strongly Disagree. Overall, parents were more satisfied with the development of ultimate outcomes then teachers. According to parents, teachers, administration and staff of The Vista School, the Competent Learner Model is most successful with development of problem solver and participator. The Competent Learner Model is least successful in development of talker, reader and writer [consistent result with Vineland Adaptive Behavior where the subscale of Written Language statistically significantly declined from the initial measure $(\mathrm{M}=7.68, \mathrm{SE}=.40)$ to two years later $(\mathrm{M}=7.23, \mathrm{SE}=.34), t$ $(74)=2.13, p=.037$,$] .$

Table 5.19

Comparison of Parents' and Teachers 'satisfaction with Ultimate Outcomes

\begin{tabular}{cclccl}
\hline & \multicolumn{2}{c}{ Parents } & & \multicolumn{2}{c}{ Teachers } \\
\hline Rank & Mean & CLM Repertoire & Rank & Mean & \multicolumn{1}{c}{ CLM Repertoire } \\
1 & 6.03 & Problem Solver & 1 & 5.00 & Problem Solver \\
2 & 6.00 & Participator & 2 & 4.96 & Participator \\
2 & 5.79 & Listener & 3 & 4.76 & Observer \\
4 & 5.70 & Observer & 4 & 4.70 & Listener \\
5 & 5.24 & Talker & 5 & 3.94 & Talker \\
6 & 5.21 & Reader & 6 & 3.93 & Reader \\
7 & 4.63 & Writer & 7 & 3.32 & Writer \\
\hline
\end{tabular}




\section{Chapter 6 \\ Discussion and Conclusion}

\section{Discussion}

In my dissertation I have suggested new directions in order to significantly advance Autism research. I have moved from identification of the problem (the stagnation of Autism research) to offering one possible explanation for the problem (conceptualization of Autism as a complex phenomenon) to one possible solution of a part of the problem (the Dynamic Evaluation Model) to testing the prototype of the Dynamic Evaluation Model using data from The Vista School.

I have explored three research questions:

RQ 1 How can we conceptualize Autism research, Autism per se, and evidence for the investigation of complex phenomena to deepen our contemporary understanding of Autism?

RQ 2 What is the best methodological tool to accelerate the assembly of nationwide evidencebased Autism treatments?

RQ 3 What is the effect of the Competent Learner Model on adaptive behavior, language development, and Autism Symptoms of children with Autism Spectrum Disorder? What is the effect of the Competent Learner Model on parental stress of parents of children with Autism?

\section{Research Question 1: Conceptualization of Autism}

Autism researchers from different disciplines have been working very hard around the globe for 71 years. Yet we still do not understand what Autism is; nor have we developed a cure. Moreover, we do not have research focused on prevention of Autism. Currently there are hundreds of treatments available (Williams \& Williams, 2011). We have behaviorally based 
treatments, psychologically based treatments, educationally based treatments, neural based treatments, medically based treatments, and biologically based treatments.

Moreover, we are not sure what is the true prevalence of Autism, nor do we understand the reasons behind its increasing trends. It seems that either we have a counting problem or Autism is truly increasing or both. I have suggested that we need to determine consistent diagnostic criteria in order to develop or adopt a consistent system for counting the frequency of Autism diagnoses.

The evidence strongly suggests that Autism research has neither uncovered the cause or causes of Autism nor conveyed understanding of the increasing prevalence of Autism nor developed a reliable evaluation system to identify effective treatments for the considerable variability in symptoms among children with Autism nor explained the complexity around the phenomenon of differential responsiveness (50\% of children with Autism benefit from the treatment but 50\% do not). From my literature review, it emerged that none of the Autism research efforts provided insights into the dynamics of Autism symptomology or the distribution of Autism symptoms vis-à-vis identification of the optimal treatment.

I suggested that the situation in Autism research is that we have not been able to agree on a definition of Autism, which has had an impact on the ways in which we have researched this phenomenon and the ways we developed treatments for Autism. This has led us into chaos in our understanding about Autism. I suggested that the chaos in Autism research was a result of the lack of conceptualization of Autism per se, complexity per se, and the way we researched them. In addition, I proposed that we were not recognizing the parts of this phenomenon and the complex interactions among the parts of the phenomenon. Accordingly, we could not reach an understanding of the enormous variability of the interacting factors that were a blueprint of 
Autism. I suggested that once we are able to uncover the connections and interactions, we will start to see reoccurring patterns and make progress in Autism research. Before that, we must precisely define the problem in order to find a solution.

I discussed that Autism research is challenged by a multifaceted problem with multiple different variables and proposed that in order to advance Autism research, we need to create a system by means of which we will start to identify and organize these variables. I suggested that we need to start to discuss and conceptualize what we are actually researching, a complex phenomenon; how we research it (research methods); what kind of understanding by way of results and findings these methods bring; and where this kind of understanding leads us.

\section{Conceptualization of Autism as a Complex Phenomenon}

I suggested that we identify Autism as a complex phenomenon; however, I did not conceptualize what this mean vis-à-vis our research. Therefore, we did not identify the optimal and productive methods to study this complex phenomenon. We did not conceptualize essential elements such as: what counts as progress, what is realistic to expect, what are the reasons behind different manifestations and intensity of symptoms. We did not answer the questions: why does each child react differently to treatment? Why do some treatments show significant gains while other treatments produce little or no gains?

I suggested that we have created too many methodological and/or epistemological structures around the way we study Autism but did not discuss the impact of these narrow structures, such as certain types of evidence, on our attempts to understand a phenomenon with a blueprint of enormous variability. I proposed that to advance our understanding of Autism as a complex phenomenon, we need first to conceptualize what actually counts as evidence and determine whether we can use the same ways of building evidence and the same types of 
evidence across different types of problems. I recommended that the character of what we considered as evidence needed to move from one-dimensional linearity to a multifactorial understanding of the context.

I advocated for expanding the boundaries of our current epistemological and methodological structures in Autism research to obtain answers that lie beyond these structures by changing our thinking, approaches, and undertaking of Autism research. I suggested that we may expand the boundaries of contemporary knowledge in Autism research by developing new research methods and/or using current research tools but in new ways.

I proposed that Autism as a complex phenomenon cannot be defined by one definition or one single explanation coming from one type of evidence. Consequently, Autism cannot be explored by use of one single research method or one single type of research result. I offered a definition of Autism that is a multi-relational (connections) and multi-dimensional (multiple quantitative and qualitative characteristics) interactive process leading to variable manifestations of Autism. I recommended searching for dimensions rather than single variables to open our research to the larger spectrum of variation.

Finally, I proposed to break down the complexity of Autism research, making it manageable and focused, by systematizing our efforts, increasing the efficiency of Autism research, and increasing the speed of research by division of etiology, symptoms, treatments, and treatment outcomes within the complex primary phenomenon of Autism. Subsequently, I suggested differentiating between Autism etiology research, in which researchers search for causes of Autism, and Autism treatment research, in which researchers seek to discover optimal treatments for a variety of symptoms. Autism etiology researchers should search for a reliable system of counting and describing the true pattern of Autism's prevalence. Autism family 
researchers should uncover the variety of ways Autism impacts family units. Finally, the Autism prevention researchers should investigate how to prevent Autism.

\section{Research Question 2: Best Methodical Tools}

In this part of my dissertation, I answered the research question (what is the best methodological tool to accelerate the assembly of nationwide evidence-based Autism treatments) by developing new methodological tool for evaluation of complex programs.

I believe that the Dynamic Evaluation Model (DEM) is an optimal methodological tool to accelerate the assembly of nationwide evidence-based Autism treatments. The DEM aims to develop a three-dimensional matrix of child Autism symptoms, treatment constellations, and behavior outcomes. I was not able to create such a matrix in this study because it requires a large data set coming from many different treatments. However, it is clear that this tool will be able to collect the necessary data to identify Autism symptoms, treatments constellations, and behavior outcomes and consequently create the three-dimensional matrix.

One of the Dynamic Evaluation Model 's purposes is to develop distributions of Autism symptoms by uncovering Autism clusters. This feature of the model depends on a large data set coming from many Autism treatments. Concerning the model's power to determine in what ways a treatment is effective or ineffective, I have a firm belief that the model is able to do that. Perhaps what would be beneficial for future improvement and use of the model is to tabulate two pairs of relationships: the relationship between the types of effective outcomes and the types of child symptoms and the relationship between the types of ineffective outcomes and types of child symptoms. In this way, the DEM would be more "user-friendly" in identifying the data and in uncovering reoccurring and interesting patterns in the data. 


\section{Summary of main findings.}

In my opinion, the development of the Dynamic Evaluation Model fulfills the requirement of comprehensive program evaluation. By uncovering clusters of Autism symptoms and by enabling understanding of the impact of "contextual" factors and of treatments structures, the Dynamic Evaluation Model can develop a three- dimensional matrix of child Autism symptoms, treatment constellations, and particular treatment outcomes.

From evaluating one entity, the Competent Learner Model at The Vista School, however, I was not able to confirm the actual capacity of the model because the capacity of the model depends on a large coordinated data set. However, from the evaluation of the Vista School I could see and experience that the model is feasible and effective to provide the necessary information to reach data- based insights regarding the effectiveness of current treatments vis-àvis child Autism symptoms.

Regarding the position of the Dynamic Evaluation Model in relation to previous research, it is clear from the literature that this type of model or guide is not available today. This may explain why Autism treatment programs are currently not comprehensively evaluated. Simply, so far there has not been any tool to comprehensively evaluate them. The Dynamic Evaluation Model is original and comprehensive; therefore, it can become a methodological tool for creating a three-dimensional matrix and for uncovering clusters of Autism symptoms. Ultimately, by uncovering these symptoms clusters, we will reduce the chaos of what we call "Autism Spectrum." Hence rather than classifying a child somewhere on the Autism spectrum, we will be able to see and say within what specific distribution of Autism symptoms the child "belongs." This breaking down of the spectrum will lead to significant improvement of our understanding of Autism treatments and the condition of Autism per se. I am positive that in 
Autism treatment research there is no guide or evaluation model of this comprehensiveness or magnitude to enable understanding of the effectiveness and/or ineffectiveness of Autism treatments.

Regarding the field of evaluation per se, the Dynamic Evaluation Model is unique in being both general and specific at the same time. This allows the model to be used for any other comprehensive evaluation of psychological or medical or educational interventions or treatments. The only item in the model that will need to be changed for such purposes is the measurement system, as each program has different objectives and will need to measure different constructs and/or behavioral outcomes. Therefore, the Dynamic Evaluation Model is a contribution to the program evaluation field as a whole in the way that it offers systematic multilevel comprehensive evaluation for comprehensive programs.

The limitation of the model. One of the rather significant limitations of this study was that I could only evaluate one particular behavioral program. The model's real power will be experienced after we are able to collect large data sets from many Autism treatments across the country. Subsequently, the model will produce what it was designed to do. It is essential, however, to make sure that phase 1: conceptualization will be feasible and easily manageable for developers of the treatment programs. Right now, the developers complete phase 1 by answering questions targeting the structure of their programs.

The aim of the conceptualization phase is to build a conceptual structure for the treatment. However, I was not able to clearly identify the structural framework of the Competent Learner Model. Therefore, I was not able to identify and describe the intervention parts and relationships among the parts. This represents a significant limitation of the model. In order to identify what part of the intervention creates what specific behavioral outcomes, the structural 
framework of the Autism treatments must be conceptualized completely, clearly and in a great detail, leading to visual and written representation.

I can only speculate as to why the developer of the Competent Learner Model was not able to answer structural framework questions. I am not sure whether the structural questions were unusual, but the developer could not provide full and clear answers. I think that one of the reasons is that this particular intervention was developed over 20 years ago. I can also speculate that the work of conceptualization is simply too difficult for program developers. Consequently, I was not able to understand the complete structural framework of the Competent Learner Model. One way or other, this conceptualization phase needs to be changed so that every developer will be able to clearly present and understand the structural framework of his or her Autism treatment. One way to facilitate this could be creation of a specific table that will help developers to visualize their program. The cells in the table will need to be constructed in such a way as to permit them to see the relationships between parts of their intervention.

Another limitation of this study was the fact that not all measures were performed measurement of the environmental context, for example. It is however a limitation of this study and not a limitation of the model per se. What would help for future nationwide evaluation of Autism treatments is to identify all measures a priori. The goal of measuring environmental context is to gauge how the intervention impacts the family, or how the family impacts the intervention. Because these measures were not administered, I could not uncover the contextual components of the Competent Learner Model and see in what ways the Competent Learner Model and families impact each other.

In conclusion, I think that the model is operable and applicable. It is necessary, however, to determine what specific measures will be used for each phase and also to develop 
environmental measures so that there can be a pilot study to ensure the reliability and validity of those environmental measures.

There are several practical implications of the model. For instance, parents will be able to quickly identify the optimal treatment for their child with such- and- such autism symptoms. This will lead to reduction of the unproductive time when the child with Autism is going from treatment to treatment. Furthermore, the parents' frustration will decrease due to quick recognition of the optimal treatment for each child on the Autism spectrum. In addition, pediatricians will be able to provide data-based evidence about optimal treatment for each individual child with her or his own cluster of Autism symptoms. Moreover, the insurance companies will be able to decrease the cost for Autism treatment. Likewise, policy makers will be able to construct or reconstruct policy in ways that will reflect the findings of this nationwide evaluation of Autism treatments. Additionally, by identifying the Autism clusters, we will be closer to a profound understanding of the complex phenomenon of Autism.

\section{Research Question 3: Effects of the Competent Learner Model}

In research question three, I studied the effect of the Competent Learner Model on adaptive behavior, language development, and Autism symptoms of children with Autism Spectrum Disorder. I also wanted to measure the effect of the Competent Learner Model on parental stress of parents of children with Autism.

Summary of main findings. The pre-test assessments of adaptive behavior were administered when the students started the program at the Vista School where the CLM was fully implemented as the daily curriculum. The post-test assessments were administered after two years of attending the Vista School. The results indicated that the Competent Learner Model was statistically significantly successful in developing four measured adaptive behavior subscales. 
Specifically, the School Community results indicate that students on average better able to use time, tools, and employ practical skills and behavior that are needed to take care of oneself. The Play and Leisure results indicate that students on average are better able to use leisure time and play. The Coping results indicate that students on average are better able to display responsibility and sensitivity toward others. Finally, the Socialization results indicate that students on the average are displaying the types of behavior and skills that are needed to get along with others.

Concerning the lessening of Autism symptoms, the results indicated that the Competent Learned Model was statistically significantly successful in developing two measured subscales of Social Interaction, which indicate that students on average are less resisting of physical contact from others, less withdrawn, less fearful, less upset when routines are changed, exhibit fewer temper tantrums, make more eye contact, imitate other people or learning activities more, are more affectionate, etc. The results for the Autism Index indicated that students on the average moved down one Autism category (have less Autism) from being very likely a person with Autism to a possibly Autistic person.

With regards to language development, the results indicated that the Competent Learner Model was statistically significantly successful in developing Auditory Comprehension and Expressive Comprehension. The Auditory expression results indicated that students on the average improved in ability to name objects and answer yes or no questions. The Expressive Comprehension indicated that students on average improved in ability to name objects and answer yes or no questions.

Parental stress results indicated that the Competent Learner Model was statistically significantly successful in reducing the overall level of parental stress. In addition, results 
indicated that parental perceptions of behavioral characteristics of their child changed in ways that positively influenced the parent-child relationship.

Interpretation of findings. As expected in light of the phenomenon of differential responsiveness, the Competent Learner Model did not produce statistically significant positive results in four measured behavioral domains. It was interesting to find that the children with the most severe form of Autism had better results than the children with less severe Autism. This indicates that the Competent Learner Model is well-suited for children with severe forms of Autism and children whose symptomology is mainly in repetitive behavior. I concluded then that children with severe Autism will benefit from the Competent Learner Model.

Significance of findings. The significance of this particular evaluation study is that the Competent Learner Model did gain specific empirical support. This comprehensive evaluation of the Competent Learner Model produced data-based evidence of its specific effectiveness. Regarding the Vista School in Pennsylvania, now parents and other stakeholders have the information available about the level of parental satisfaction with the School and the effectiveness of their program.

\section{Future Research}

Regarding The Competent Learner Model, the full structural framework needs to be developed. The measurement of the degree of provider engagement at The Vista School and the assessments of all contextual factors need to be completed.

Further research can target and explain the reasons behind a negative strong correlational pattern (the more adaptive behavior is developed, the less the stereotyped behavior) suggesting that The Competent Learner Model could be a good intervention for children with a large deficit 
and strong intensity of stereotyped behavior. If more studies confirm this correlational pattern, research toward establishment of causation should take place.

Future research can also be done based on rich data coming from the specialized demographics of Autism. These data can be a source of new and interesting hypotheses, research questions, and relationships. Researchers may wish to explore, for example, why parents with technical education tend to have children with Autism. Alternatively, they may wish to map the number of treatments and medication a child with Autism on average underwent.

I suggest continuing and deepening the evaluation of the Competent Learner Model in the Vista School Pennsylvania and elsewhere. To my knowledge, the Competent Learner Model has been implemented in many locations worldwide. I suggest evaluating every CLM implementation site to create an even richer picture of this particular Autism program.

Moreover, I suggest starting nationwide evaluation of Autism treatments. Obviously future research needs to be done to uncover the etiology of Autism and create a theory of its causation. Further research should address the development of new research methods or tools or approaches that will be strong and powerful enough to uncover the relationship between the symptoms and the cause or causes for Autism.

It is necessary to admit that current research methods are not powerful enough, strong enough or insightful enough to get a full understanding of the dynamics of complex phenomena. In addition, we need to remain open to many research methodologies, methods and perspectives and not to base our research on a single epistemological foundation. Particularly noteworthy is that Autism research is predominantly done via quantitative means, and qualitative research is rarely employed for investigating Autism or its treatments. It will be necessary to leave the Cold War of epistemological camps. Given the enigma that Autism is, we will simply have to use 
everything at our disposal to uncover the fundamental constantly changing complexity of Autism. 


\section{References}

Amaral, D. G. (2011). The promise and the pitfalls of autism research: An introductory note for new autism researchers. Brain Research, 13803-9. doi:10.1016/j.brainres.2010.11.077

American Psychiatric Association. (1952). Diagnostic and statistic manual: Mental disorders. Washington, DC: American Psychiatric Association: Mental Hospital Service.

American Psychiatric Association. (1968). Diagnostic and statistic manual of mental disorders (2nd ed.). Washington, DC: Author.

American Psychiatric Association. (1980). Diagnostic and statistical manual of mental disorders (3rd ed.). Washington, DC: Author.

American Psychiatric Association (1987). Diagnostic and statistical manual of mental disorders (3rd ed., rev.). Washington, DC: Author.

American Psychiatric Association. (1994). Diagnostic and statistical manual of mental disorders (4th ed.). Washington, DC: Author.

American Psychiatric Association (2013). DSM-V Implementation and Support Retrieved January 21, 2014 from http://www.dsm5.org/Pages/Default.aspx

Autism Speaks (2011). http://www.autismspeaks.org/what-autism/facts-about-autism

Aylward, E.H., Minshew, N. J., \& Field, K. (2001). Brain volume in autism. Journal of Child Neurology, 16(6), 421-424.

Baron-Cohen, S. (2004). Autism: Research into causes and intervention. Pediatric Rehabilitation, 7(2), 73-78.

Bogin, J. (2008). Overview of discrete trial training. Sacramento, CA: National Professional Development Center on Autism Spectrum Disorders, M.I.N.D. Institute, The University of California at Davis Medical School. 
Brittingham, B., O’Brien, P. M., \& Alig, J. (2008). Accreditation and institutional research: The traditional role and new dimensions. New Directions for Higher Education, 141, 69-76. doi:10.1002/he.294.

Brookman, L., Boettcher, M., Klein, E., Openden, D., Koegel, R. L., \& Koegel, L. (2003). Facilitating Social Interactions in a Community Summer Camp Setting for Children with Autism. Journal Of Positive Behavior Interventions, 5(4), 249-252. doi:10.1177/10983007030050040801

Bryson, S. E., Koegel, L. K., Koegel, R. L., Openden, D., Smith, I. M., \& Nefdt, N. (2007). Large scale dissemination and community implementation of pivotal response treatment: Program description and preliminary data. Research \& Practice For Persons With Severe Disabilities, 32(2), 142-153.

Carr, J. E., \& Firth, A. M. (2005). The Verbal Behavior Approach to Early and Intensive Behavioral Intervention for Autism: A Call for Additional Empirical Support. Journal Of Early And Intensive Behavior Intervention, 2(1), 18-27.

Chasson, G. S., Harris, G. E., \& Neely, W. J. (2007). Cost Comparison of Early Intensive Behavioral Intervention and Special Education for Children with Autism. Journal of Child And Family Studies, 16(3), 401-413.

Coggon, D., Rose, G. A., \& Barker, D. J. P. (1997). Epidemiology for the uninitiated. London: BMJ Pub. Group.

Cooksy, L. J., Gill, P., \& Kelly, P. A. (2001). The program logic model as an integrative framework for a multimethod evaluation. Evaluation and Program Planning, 24(2), 119128. 
Coolican, J., Smith, I. M., \& Bryson, S. E. (2010). Brief parent training in pivotal response treatment for preschoolers with autism Jamesie Coolican, Isabel M. Smith, and Susan E. Bryson Parent training in PRT. Journal of Child Psychology \& Psychiatry, 51(12), 13211330. doi:10.1111/j.1469-7610.2010.02326.x

Dicker, S. (2013). Entering the Spectrum. Infants \& Young Children: An Interdisciplinary Journal of Special Care Practices, 26(3), 192-203. doi:10.1097/IYC.0b013e3182953081

Didden, R., Sigafoos, J., Lang, R., O'Reilly, M., Drieschner, K., \& Lancioni, G. E. (2012). Intellectual disabilities. In P. Sturmey, M. Hersen (Eds.), Handbook of evidence-based practice in clinical psychology, Vol 1: Child and adolescent disorders (pp. 131-159). Hoboken, NJ US: John Wiley \& Sons Inc. doi:10.1002/9781118156391.ebcp001006

Fienup, D. M., \& Doepke, K. (2008). Evaluation of a Changing Criterion Intervention to Increase Fluent Responding with an Elementary Age Student with Autism. International Journal of Behavioral Consultation \& Therapy, 4(3), 297-303.

Flaherty, D. K. (2011). The vaccine-autism connection: A public health crisis caused by unethical medical practices and fraudulent science. Annals of Pharmacotherapy, 45(10), 1302-1304.

Folstein, S.E. (1999). Autism. International Review of Psychology, 11(4), 269-277.

Eldevik, S., Hastings, R. P., Hughes, J., Jahr, E., Eikeseth, S., \& Cross, S. (2009). Meta-Analysis of Early Intensive Behavioral Intervention for Children With Autism. Journal of Clinical Child \& Adolescent Psychology, 38(3), 439-450. doi:10.1080/15374410902851739 [[ Alternative Title: L'utilisation des données provenant des participants pour améliorer les interventions comportementales intensives basées sur des données probantes pour les enfants autistes.]] 
Eldevik, S., Hastings, R. P., Hughes, J., Jahr, E., Eikeseth, S., \& Cross, S. (2010). Using participant data to extend the evidence base for intensive behavioral intervention for children with autism. American Journal On Intellectual And Developmental Disabilities, 115(5), 381-405. doi:10.1352/1944-7558-115.5.381

Granpeesheh, D., Tarbox, J., \& Dixon, D. R. (2009). Applied behavior analytic interventions for children with autism: A description and review of treatment research. Annals of Clinical Psychiatry (Taylor \& Francis Ltd), 21(3), 162-173.

Gresham, F. M., \& MacMillan, D. L. (1998). Early Intervention Project: Can Its Claims Be Substantiated and Its Effects Replicated? Journal Of Autism \& Developmental Disorders, $28(1), 5-13$.

Gresham, F. M., \& MacMillan, D. L. (1997). Denial and defensiveness in the place of fact and reason: Rejoinder to Smith and Lovaas. Behavioral Disorders, 22(4), 219-230.

Gurkan, C., \& Hagerman, R. J. (2012). Targeted Treatments in Autism and Fragile X Syndrome. Research In Autism Spectrum Disorders, 6(4), 1311-1320.

Hayes, S. (2009). A Look at Asperger's Syndrome. Scholastic Parent \& Child, 17(2), 86-89.

Herman A. (1996). Neurobiological insight into infantile autism. The Harvard Brain, Spring, 1925.

Hughes, J. R., \& Melyn, M. (2005). EEG and seizures in autistic children and adolescents: Further findings with the therapeutic implication. Clinical EEG Neuroscience, 36(1), 1520.

Isaacs, B., Clark, C., Correia, S., \& Flannery, J. (2009). Utility of Logic Models to Plan Quality of Life Outcome Evaluations. Journal of Policy \& Practice In Intellectual Disabilities, 6(1), 52-61. doi:10.1111/j.1741-1130.2008.00197.x 
Johnson, D. F. (1977). Behaviorism Examines its 'Roots' and its Flowers. Psyccritiques, 22(8), 552-554. doi:10.1037/016142

Johnson, N. (2009). Simply Complexity: A clear guide to complexity theory. One world Publications.

Kanner, L., (1943). Autistic disturbances of affective contact. Nervous Child, (2), 217-250

Kasari, C. (2002). Assessing change in early intervention programs for children with autism. Journal of Autism and Developmental Disorders, 32, 447-461.

Koegel, L., Ashbaugh, K., Koegel, R. L., Detar, W. J., \& Regester, A. (2013). INCREASING SOCIALIZATION IN ADULTS WITH ASPERGER'S SYNDROME. Psychology In The Schools, 50(9), 899-909. doi:10.1002/pits.21715

Koegel, L., Park, M., \& Koegel, R. (2013). Using Self-Management to Improve the Reciprocal Social Conversation of Children with Autism Spectrum Disorder. Journal of Autism And Developmental Disorders,

Koegel, R., Bradshaw, J., Ashbaugh, K., \& Koegel, L. (2013). Improving Question-Asking Initiations in Young Children with Autism Using Pivotal Response Treatment. Journal of Autism And Developmental Disorders,

Koegel, R., Kim, S., Koegel, L., \& Schwartzman, B. (2013). Improving socialization for high school students with ASD by using their preferred interests. Journal of Autism And Developmental Disorders, 43(9), 2121-2134. doi:10.1007/s10803-013-1765-3

Koegel, R., Bharoocha, A., Ribnick, C., Ribnick, R., Bucio, M., Fredeen, R., \& Koegel, L. (2012). Using individualized reinforcers and hierarchical exposure to increase food flexibility in children with autism spectrum disorders. Journal of Autism \& Developmental Disorders, 42(8), 1574-1581. doi:10.1007/s10803-011-1392-9 
Koegel, L., Koegel, R. L., Green-Hopkins, I., \& Barnes, C. (2010). Brief Report: QuestionAsking and Collateral Language Acquisition in Children with Autism. Journal of Autism \& Developmental Disorders, 40(4), 509-515. doi:10.1007/s10803-009-0896-Z

Koegel, L., \& LaZebnik, C. (2009). Growing up on the spectrum: A guide to life, love, and learning for teens and young adults with autism and Asperger's. New York, NY US: Viking.

Koegel, R. L., \& Koegel, L. (2006). Pivotal response treatments for autism: Communication, social, \& academic development. Baltimore, MD US: Paul H Brookes Publishing.

Koegel, L., Koegel, R. L., Nefdt, N., Fredeen, R., Klein, E. F., \& Bruinsma, Y. M. (2005). First S.T.E.P.: A Model for the Early Identification of Children With Autism Spectrum Disorders. Journal of Positive Behavior Interventions, 7(4), 247-252.

Koegel, R. L., Symon, J. B., \& Kern Koegel, L. (2002). Parent education for families of children with autism living in geographically distant areas. Journal of Positive Behavior Interventions, 4(2), 88-103. doi:10.1177/109830070200400204

Koegel, L. K., Koegel, R. L., Shoshan, Y., \& McNerney, E. (1999). Pivotal Response Intervention II: Preliminary long-term outcome data. The Journal of the Association for Persons with Severe Handicaps, 24,186-198.

Koegel, L., Camarata, S., Valdez-Menchaca, M., \& Koegel, R. (1998). Setting generalization of question-asking by children with autism. American Journal Of Mental Retardation: AJMR, 102(4), 346-357.

Koegel, R. L., Koegel, L., \& Parks, D. (1995). 'Teach the Individual' Model of Generalization: Autonomy Through Self-Management. In R. L. Koegel, L. Koegel (Eds.), Teaching 
children with autism: Strategies for initiating positive interactions and improving learning opportunities (pp. 67-77). Baltimore, MD US: Paul H Brookes Publishing. Koegel, R. L., Koegel, L., Frea, W. D., \& Smith, A. E. (1995). Emerging Interventions for Children with Autism: Longitudinal and Lifestyle Implications. In R. L. Koegel, L. Koegel (Eds.), Teaching children with autism: Strategies for initiating positive interactions and improving learning opportunities (pp. 1-15). Baltimore, MD US: Paul H Brookes Publishing.

Koegel, R. L., \& Koegel, L. (1995). Teaching children with autism: Strategies for initiating positive interactions and improving learning opportunities. Baltimore, MD US: Paul H Brookes Publishing.

Koegel, L., Koegel, R., Hurley, C., \& Frea, W. (1992). Improving social skills and disruptive behavior in children with autism through self-management. Journal of Applied Behavior Analysis, 25(2), 341-353.

Koegel, R., \& Egel, A. (1979). Motivating autistic children. Journal of Abnormal Psychology, 88(4), 418-426.

Levy, S. E., Mandell, D. S., \& Schultz, R. T. (2009). Autism. Lancet, 374(9701), 1627-1638. doi:10.1016/S01406736(09)61376-6

Lovaas, O. (1987). Behavioral treatment and normal educational and intellectual functioning in young autistic children. Journal of Consulting And Clinical Psychology, 55(1), 3-9. doi:10.1037/0022-006X.55.1.3

Lovaas, O. I., \& Wright, S. (2006). " A Reply to Recent Public Critiques...". Journal of Early \& Intensive Behavior Intervention, 3(2). 
Lozowski-Sullivan, S. (2012). Psychometric properties of diagnostic assessment instruments for autism spectrum disorders in a community sample aged 2-17 years. Dissertation Abstracts International, 72,

Maurice, C. E., Green, G. E., \& Luce, S. C. (1996). Behavioral intervention for young children with autism: A manual for parents and professionals. Pro-ed.

Mceachin, J., Smith, T., \& Lovaas, O. (1993). Long-term outcome for children with autism who received early intensive behavioral treatment. American Journal on Mental Retardation, 97(4), 359-372

Millward, C., Ferriter, M., Calver, S., \& Connell-Jones, G. (2008). Gluten-and casein-free diets for autistic spectrum disorder. Cochrane Database Syst Rev, 2.

Multi-Health Systems Inc., (2013). Retrieved from http://www.mhs.com/product.

Nanclares, V. (2004). Program evaluation: An analysis of the puentes intensive home-based treatment program for young children with autism. Dissertation Abstracts International, 65

National Inst. of Child Health and Human Development (NIH), B. D. (2005). Autism Overview: What We Know. National Institutes Of Health

Odom, S. L., Boyd, B. A., Hall, L. J., \& Hume, K. (2010). Evaluation of comprehensive treatment models for individuals with autism spectrum disorders. Journal of Autism \& Developmental Disorders, 40(4), 425-436. doi:10.1007/s10803-009-0825-1

Park, C., \& National Society for Autistic Children, S. Y. (1971). Research and Education: Top Priorities for Mentally Ill Children. Proceedings of the Second Annual Meeting and Conference of the National Society for Autistic Children (San Francisco, California, June 24-27, 1970). 
Peterson, G. B., Larsson, E. V., \& Riedesel, K. L. (2003). A Conceptual Toolkit for Intensive Early Behavioral Intervention Teachers. Journal of Behavioral Education, 12(2), 131146.

Pope, K. L. (1999). Autism: An Overview and Investigation into the Lovaas Intervention Technique.

Reichow, B., Volkmar, F., \& Cicchetti, D. (2008). Development of the evaluative method for evaluating and determining evidence based practices in autism. Journal of Autism and Developmental Disorders, 38, 1311-1319.

Romanczyk, R. G., Weiner, T., Lockshin, S., \& Ekdahl, M. (1999). Research in autism: Myths, controversies, and perspectives. In D. Zager (Ed.), Autism: Identification, education, and treatment (2nd ed.) (pp. 23-61). Mahwah, NJ US: Lawrence Erlbaum Associates Publishers.

Rosenblatt, J., Bloom, P., \& Koegel, R. L. (1995). Over selective Responding: Description, Implications, and Intervention. In R. L. Koegel, L. Koegel (Eds.), Teaching children with autism: Strategies for initiating positive interactions and improving learning opportunities (pp. 33-42). Baltimore, MD US: Paul H Brookes Publishing.

Ryan, C. S., \& Ilemmes, N. S. (2005). Post-training discrete-trial teaching performance by instructors of young children with autism in early intensive behavioral intervention. Behavior Analyst Today, 6(1), 1-12.

Schreibman, L. (2008). Treatment Controversies in Autism. Zero To Three, 28(4), 38-45.

Schreibman, L., \& Koegel, R. L. (2005). Training for Parents of Children With Autism: Pivotal Responses, Generalization, and Individualization of Interventions. In E. D. Hibbs, P. S. Jensen (Eds.), Psychosocial treatments for child and adolescent disorders: Empirically 
based strategies for clinical practice (2nd ed.) (pp. 605-631). Washington, DC US: American Psychological Association.

Sherer, M. (2003, April). Individual behavioral profiles and predictors of treatment effectiveness for children with autism. Dissertation Abstracts International, 63,

Skinner, B.F (1957). Verbal behavior. Englewood Cliffs, NJ: Prentice-Hall.

Smith, T. (2001). Discrete Trial Training in the Treatment of Autism. Focus On Autism And Other Developmental Disabilities, 16(2), 86-92.

Smith, T., Groen, A. D., \& Wynn, J. W. (2000). Randomized Trial of Intensive Early Intervention for Children With Pervasive Developmental Disorder. American Journal on Mental Retardation, 105(4), 269-285.

Smith, I. M., Koegel, R. L., Koegel, L. K., Openden, D. A., Fossum, K. L., \& Bryson, S. E. (2010). Effectiveness of a Novel Community-Based Early Intervention Model for Children With Autistic Spectrum Disorder. American Journal On Intellectual \& Developmental Disabilities, 115(6), 504-523. doi:10.1352/1944-7558-115.6.504

Sparrow, S., Balla, D., \& Cicchetti, D. (1984) American Guidance Service, Inc.: 4201 Woodland Rd.Circle Pines, MN 55014. Retrieved from https://www.pearsonassessments.com Steyaert, J. G., \& De La Marche, W. (2008). What's new in autism?. European Journal Of Pediatrics, 167(10), 1091-1101. doi:10.1007/s00431-008-0764-4

Tarbox, J., Wilke, A. E., Findel-Pyles, R. S., Bergstrom, R. M., \& Granpeesheh, D. (2010). A comparison of electronic to traditional pen-and-paper data collection in discrete trial training for children with autism. Research In Autism Spectrum Disorders, 4(1), 65-75. doi:10.1016/j.rasd.2009.07.008 
Tchaconas, A., \& Adesman, A. (2013). Autism spectrum disorders: A pediatric overview and update. Current Opinion In Pediatrics, 25(1), 130-144.

doi:10.1097/MOP.0b013e32835c2b70

Thomson, K., Martin, G. L., Arnal, L., Fazzio, D., \& Yu, C. T. (2009). Instructing Individuals to Deliver Discrete-Trials Teaching to Children with Autism Spectrum Disorders: A Review. Research In Autism Spectrum Disorders, 3(3), 590-606.

Tucci, V., Hursh, D., \& Laitinen, R. (2004). The competent learner model: A merging of applied behavior analysis, direct instruction, and precision teaching. Evidence-Based Educational Methods (109-123). San Diego, CA US: Elsevier Academic Press. DOI:10.1016/B978012506041-7/50009-7.

Tucci, V., \& Hursh, D. (1991). Competent Learner Model: Instructional programming for teachers and learners. Education \& Treatment Of Children (ETC), 14(4), 349.

Ventola, P. (2013). Review of 'The PRT pocket guide: Pivotal response treatment for autism spectrum disorders'. Journal of Autism And Developmental Disorders, 43(1), 247-248. doi:10.1007/s10803-012-1572-2

Vernon, T., Koegel, R., Dauterman, H., \& Stolen, K. (2012). An early social engagement intervention for young children with autism and their parents. Journal of Autism \& Developmental Disorders, 42(12), 2702-2717. doi:10.1007/s10803-012-1535-7

Volkmar, F., Lord, C., Bailey, A., Schultz, R., \& Klin, A. (2004). Autism and pervasive developmental disorders. Journal of Child Psychology And Psychiatry, And Allied Disciplines, 45(1), 135-170.

Voress, J. K. (2005). From the Periodicals Director. Journal of Positive Behavior Interventions, $7(2), 66$. 
Wakefield, A. J., Murch, S. H., Anthony, A., Linnell, J., Casson, D. M., Malik, M., ... \& WalkerSmith, J. A. (1998). RETRACTED: Ileal-lymphoid-nodular hyperplasia, non-specific colitis, and pervasive developmental disorder in children. The Lancet, 351(9103), 637641.

Warash, B., Curtis, R., Hursh, D., \& Tucci, V. (2008). Skinner meets Piaget on the Reggio playground: Practical synthesis of applied behavior analysis and developmentally appropriate practice orientations. Journal of Research in Childhood Education, 22(4), 441-453.

Warren, Z., McPheeters, M. L., Sathe, N., Foss-Feig, J. H., Glasser, A., \& VeenstraVanderWeele, J. (2011). A systematic review of early intensive intervention for autism spectrum disorders. Pediatrics, 127(5), e1303-e1311. doi:10.1542/peds.2011-0426

Weaver, W. (1991). Science and complexity. In Facets of Systems Science (pp. 449-456). Springer US.

Weiss, M. J., \& Demiri, V. (2011). Jumpstarting Communication Skills in Children with Autism: A Parents Guide to Applied Verbal Behavior. Woodbine House.

Wilczynski, S. M., Fisher, L., Sutro, L., Bass, J., Mudgal, D., Zeiger, V., \& ... Logue, J. (2011). Evidence-based practice and autism spectrum disorders. In M. A. Bray, T. J. Kehle (Eds.), The Oxford handbook of school psychology (pp. 567-592). New York, NY US: Oxford University Press.

Williams, B.F. \& Williams, R.L. (2011) Effective programs for treating autism spectrum disorder: Applied behavior analysis models. New York, Routledge.

Williams, J. G., Higgins, J. P., \& Brayne, C. E. (2006). Systematic review of prevalence studies of autism spectrum disorders. Archives of disease in childhood, 91(1), 8-15. 
Wing, L., \& Potter, D. (2002). The epidemiology of autistic spectrum disorders: is the prevalence rising? Mental Retardation and Developmental Disabilities Research Reviews, 8(3), 151161.

Young, H. (2007). An examination of the variables that affect the outcomes of children with autism spectrum disorders. Dissertation Abstracts International Section A, 68,

Zhao, X., Leotta, A., Kunstanovich, V., Lajonchere, C., Genschwind, D. H., Law, K., Law, P.,Qiu, S.,Lord, C., Sebat, J., Ye, K., \&Wingler, M. (2007). A unified genetic theory for sporadic and inherited autism. Proceedings of the National Academy of Science of the United Stated of America, 104(31), 12831-12836. 
Table A1/1

\section{Appendix 1}

Examination of the Normality Assumption: Kolomogorov-Smirnov Test

\begin{tabular}{|c|c|c|c|c|c|c|}
\hline \multicolumn{7}{|c|}{ Tests of Normality } \\
\hline & \multicolumn{3}{|c|}{ Kolmogorov-Smirnov ${ }^{\mathrm{a}}$} & \multicolumn{3}{|c|}{ Shapiro-Wilk } \\
\hline & Statistic & df & Sig. & Statistic & df & Sig. \\
\hline DP1 & .091 & 75 & $.200^{*}$ & .991 & 75 & .884 \\
\hline DP2 & .165 & 75 & .000 & .940 & 75 & .002 \\
\hline DP3 & .267 & 75 & .000 & .881 & 75 & .000 \\
\hline DP4 & .170 & 75 & .000 & .949 & 75 & .004 \\
\hline DP5 & .078 & 75 & $.200^{*}$ & .989 & 75 & .772 \\
\hline DP6 & .195 & 75 & .000 & .896 & 75 & .000 \\
\hline DP7 & .139 & 49 & .019 & .968 & 49 & .203 \\
\hline DP8 & .157 & 75 & .000 & .958 & 75 & .014 \\
\hline DP9 & .164 & 49 & .002 & .929 & 49 & .006 \\
\hline DP10 & .139 & 75 & .001 & .973 & 75 & .104 \\
\hline DP11 & .067 & 75 & $.200 *$ & .983 & 75 & .421 \\
\hline DP12 & .167 & 75 & .000 & .951 & 75 & .006 \\
\hline DP13 & .193 & 75 & .000 & .922 & 75 & .000 \\
\hline DP14 & .162 & 75 & .000 & .947 & 75 & .003 \\
\hline DP15 & .072 & 75 & $.200 *$ & .987 & 75 & .665 \\
\hline DP16 & .139 & 49 & .019 & .974 & 49 & .360 \\
\hline DP17 & .143 & 49 & .014 & .956 & 49 & .067 \\
\hline DP18 & .138 & 31 & .137 & .961 & 31 & .313 \\
\hline DP19 & .171 & 33 & .016 & .883 & 33 & .002 \\
\hline DP20 & .203 & 33 & .001 & .886 & 33 & .002 \\
\hline DP21 & .206 & 20 & .026 & .939 & 20 & .227 \\
\hline DP22 & .203 & 33 & .001 & .886 & 33 & .002 \\
\hline DP23 & .153 & 39 & .022 & .869 & 39 & .000 \\
\hline DP24 & .078 & 39 & $.200 *$ & .990 & 39 & .977 \\
\hline DP25 & .158 & 17 & $.200 *$ & .914 & 17 & .118 \\
\hline DP26 & .128 & 15 & $.200 *$ & .937 & 15 & .348 \\
\hline DP27 & .148 & 14 & $.200 *$ & .941 & 14 & .433 \\
\hline DP28 & .196 & 14 & .150 & .939 & 14 & .401 \\
\hline DP29 & .112 & 14 & $.200 *$ & .964 & 14 & .787 \\
\hline
\end{tabular}


Figure A1/2

Examination of the Normality Assumption: Normal Q-Q Plots. Figure illustrates the patters in the distributions
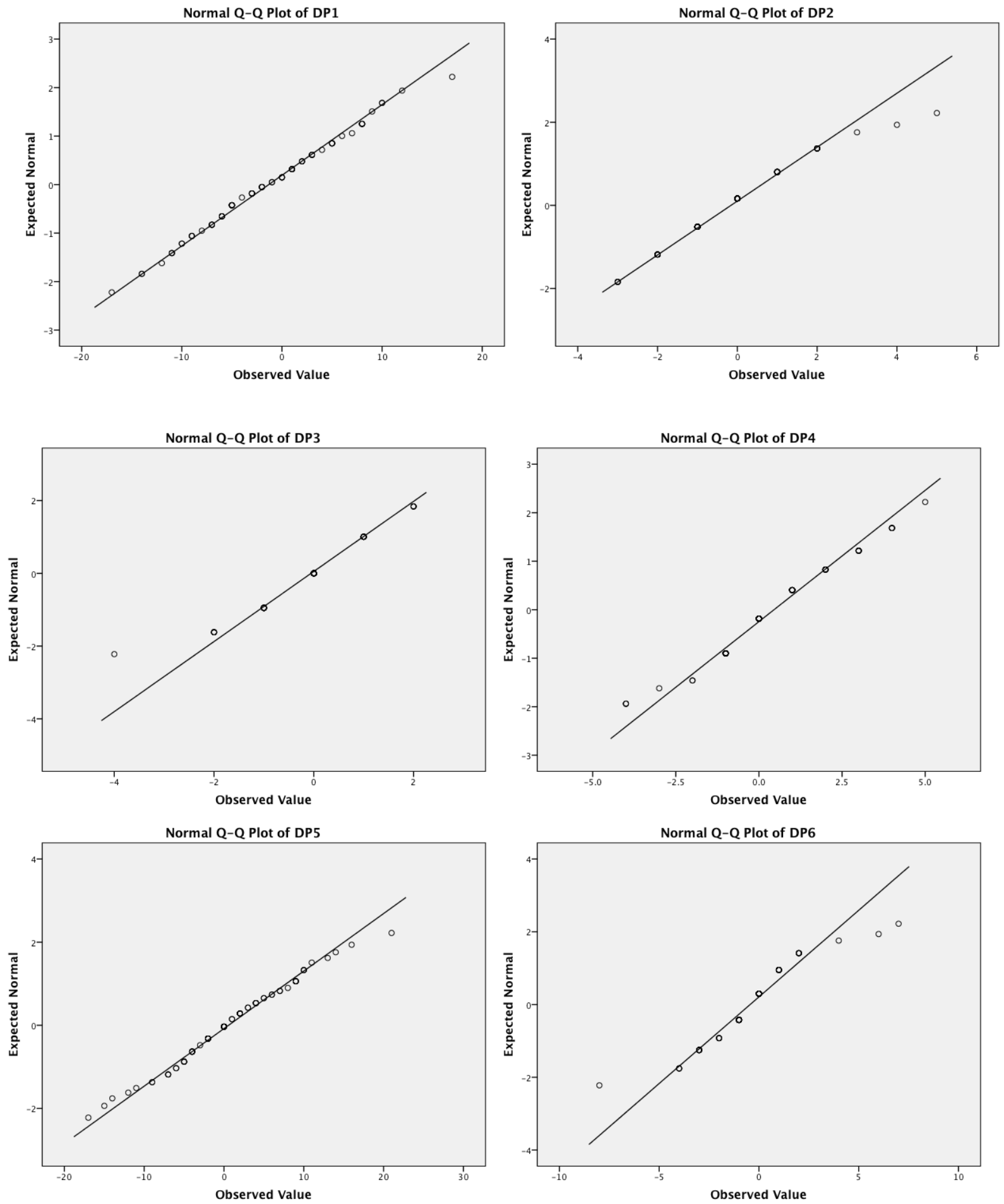

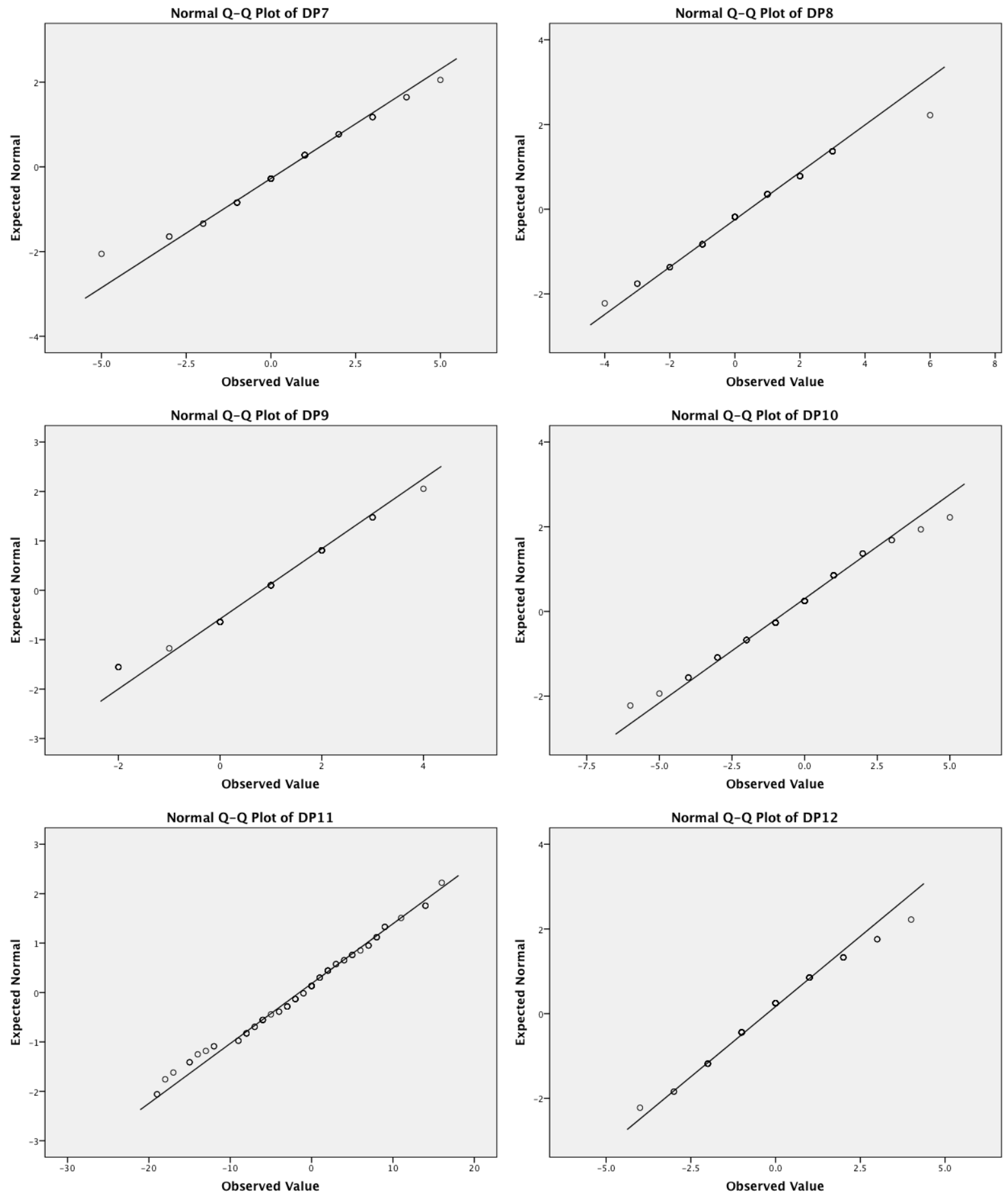

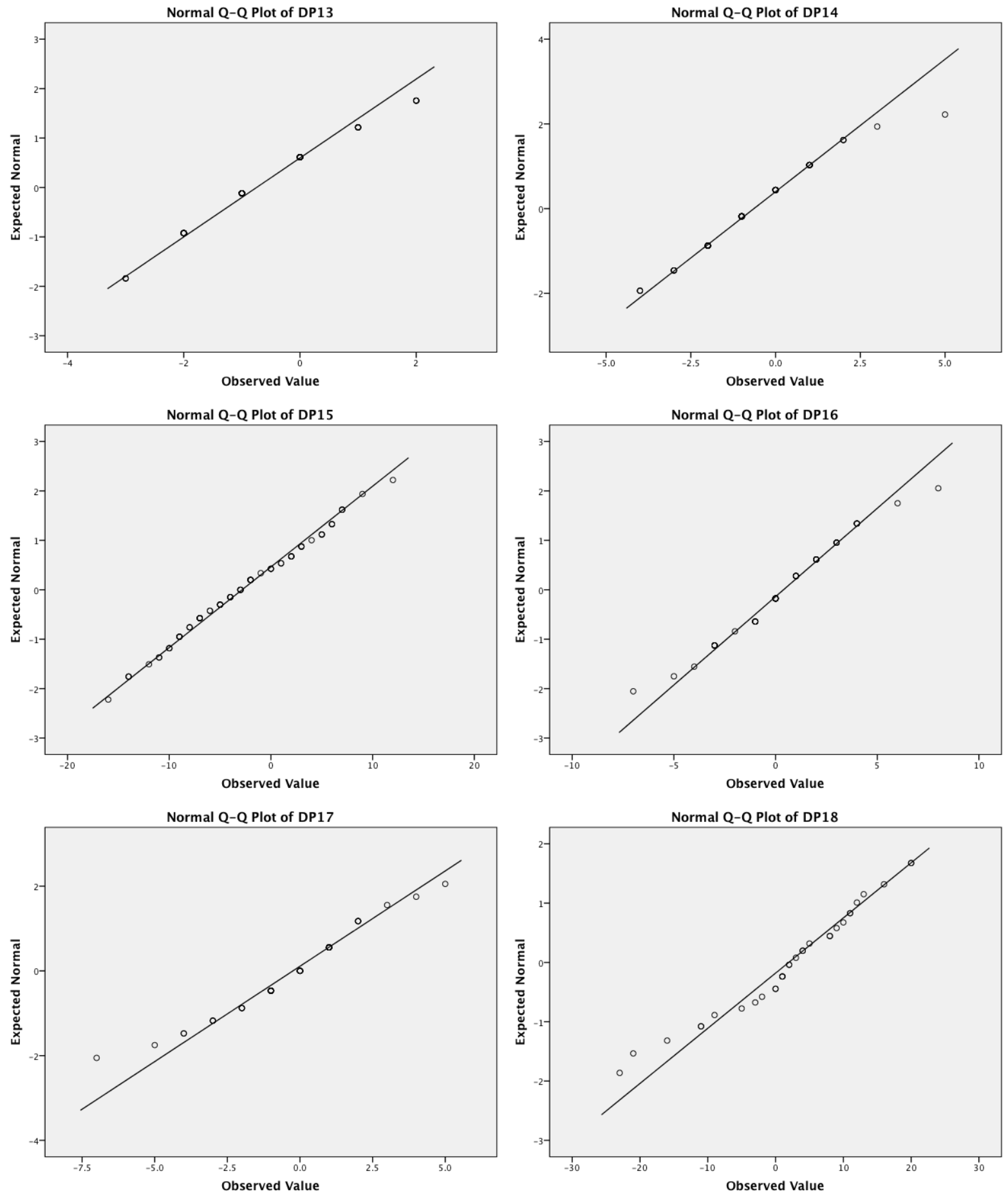

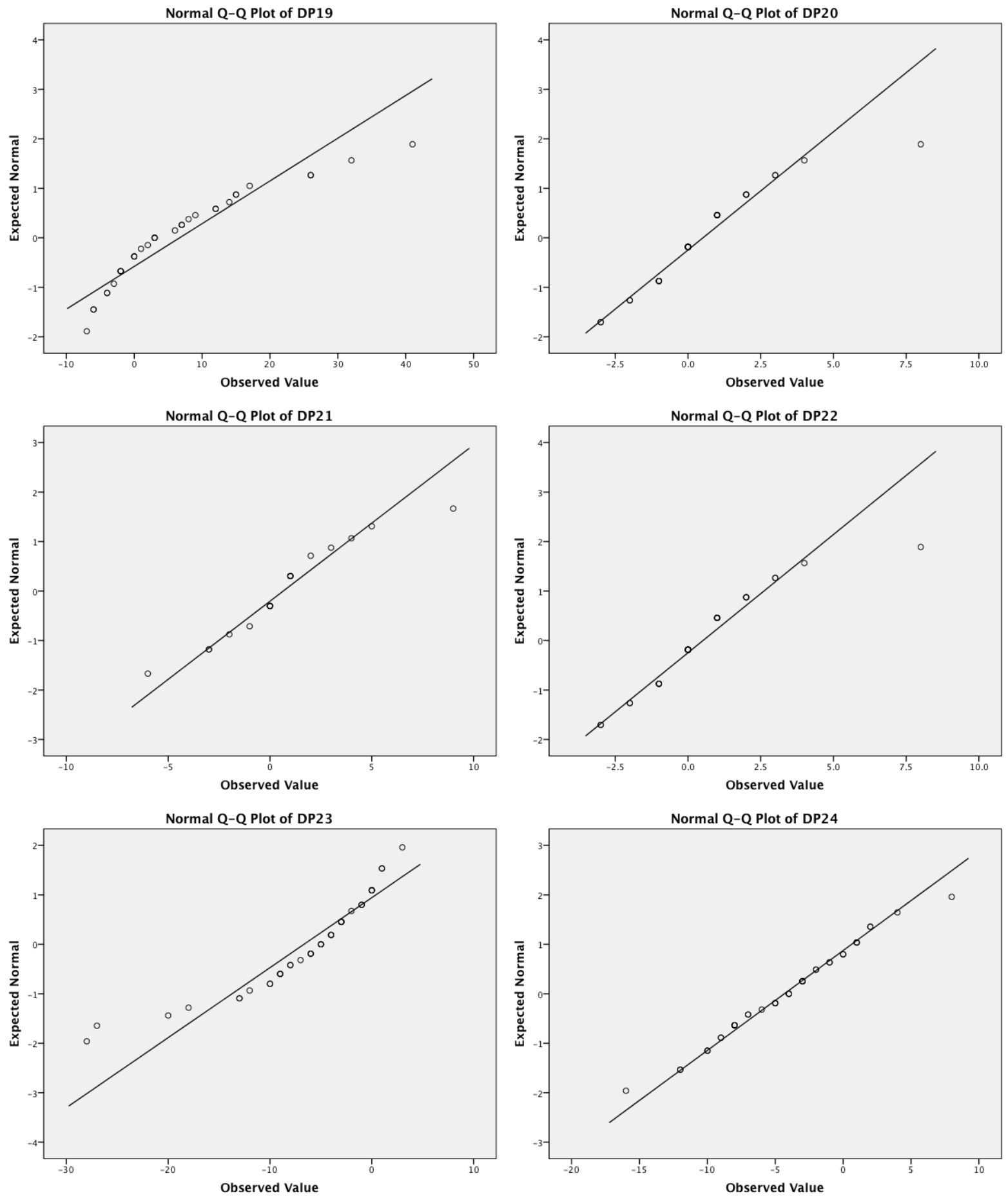

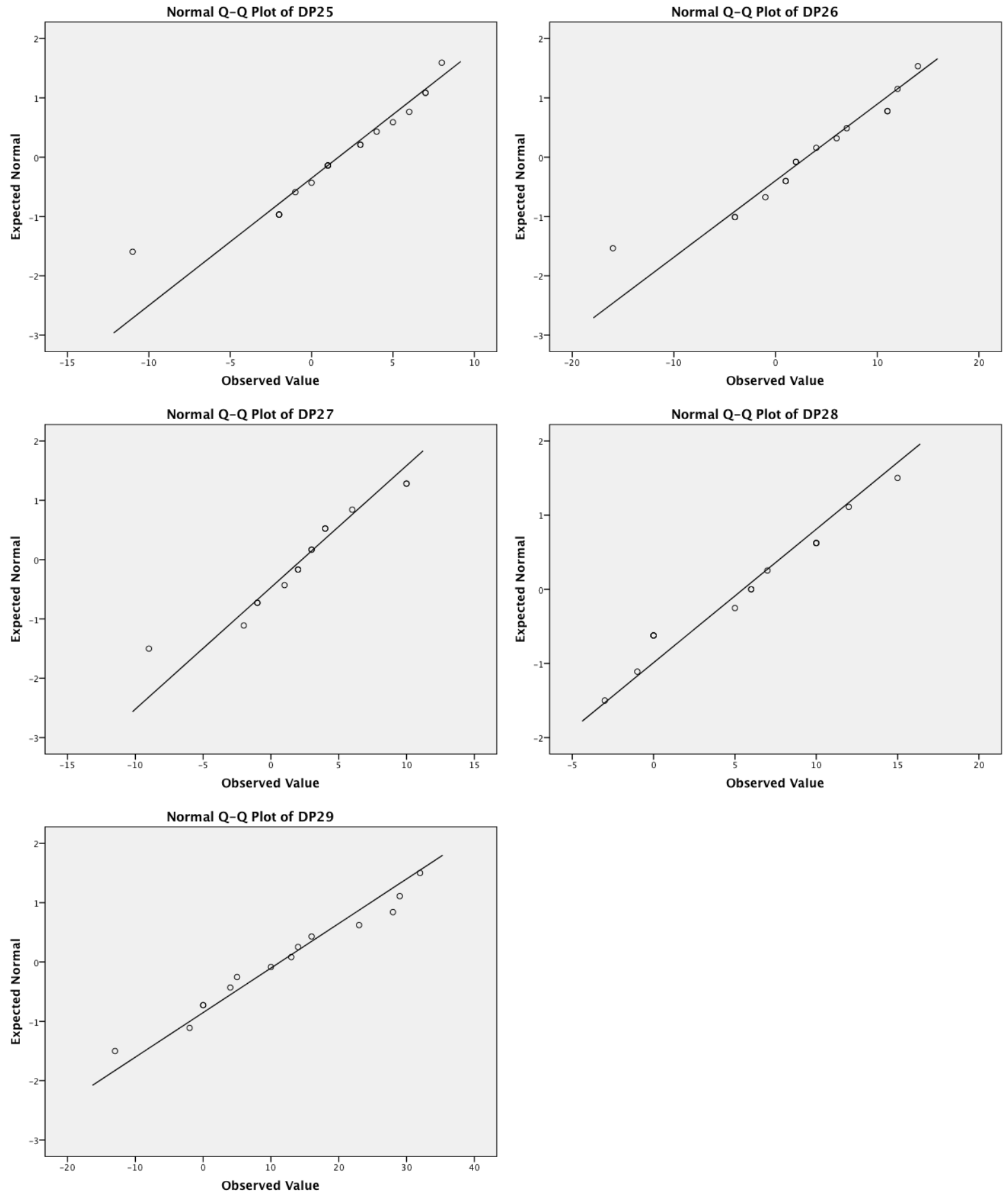


\section{Appendix 2}

Table A2/1

Category A: Comprehensive Evaluation of Autism Behavioral Based Treatment

\begin{tabular}{clc}
\hline Category & \multicolumn{1}{c}{ Autism Treatment } & $\begin{array}{c}\text { Comprehensive } \\
\text { Program Evaluations }\end{array}$ \\
\hline A1 & The Lovaas Institute: Discrete Trials Training & NO \\
A2 & The Koegel Center: Pivotal Response Training & NO \\
A3 & Strategic Teaching and Reinforcement System: Verbal Behavior & NO \\
A4 & Project Data: School-Based Inclusion Model & NO \\
A5 & May Institute: System of Care & NO \\
A6 & New England Center for Children: Teaching Independence & NO \\
A7 & Princeton Child Development Institute: Across the Lifespan & NO \\
A8 & Judge Rotenberg Center: Zero Exclusion & NO \\
A9 & Autism Partnership Seal Beach & NO \\
A10 & Center for Autism and Related Disorders (CARD) & NO \\
A11 & Alpine Learning Group Paramus & NO \\
A12 & Eden Institute Princeton & NO \\
A13 & Douglass Developmental Disabilities Center & NO \\
A14 & Institute for Child Development - SUNY & NO \\
A15 & Pyramid Approach to Education & NO \\
A16 & Strategies for Teaching based on Autism Research (STAR) ? & NO \\
A17 & Summit Academy & NO \\
A18 & Therapeutic Pathways & NO \\
A19 & Valley Program & NO \\
A20 & Children's Toddler School & NO \\
A21 & Walden Model & NO \\
A22 & Preschoolers and Parents (LEAP) & \\
& & \\
\hline
\end{tabular}


Table A2/2

Category B: Comprehensive Evaluation of Autism Psychological Based Treatment

\begin{tabular}{clc}
\hline Category & \multicolumn{1}{c}{ Treatment } & $\begin{array}{c}\text { Comprehensive Program } \\
\text { Evaluation }\end{array}$ \\
\hline B1 & Developmental, Individual Difference - Floortime & NO \\
B2 & The Son-Rise & NO \\
B3 & Gentle Teaching & NO \\
B4 & Holding Therapy & \\
\hline
\end{tabular}

Table A2/3

Category C: Comprehensive Evaluation of Autism Educational Based Treatment

\begin{tabular}{clc}
\hline Category & \multicolumn{1}{c}{ Treatment } & $\begin{array}{c}\text { Comprehensive Program } \\
\text { Evaluation }\end{array}$ \\
\hline C1 & $\begin{array}{l}\text { Treatment of Autistic and Related Communication-Handicapped } \\
\text { Children }\end{array}$ & NO \\
C2 & $\begin{array}{l}\text { Learning Experiences: An Alternative Program for Preschoolers } \\
\text { and Parents }\end{array}$ & NO \\
\hline
\end{tabular}

Table A2/4

Category D: Comprehensive Evaluation of Autism Neural Based Treatment

\begin{tabular}{clc}
\hline Category & \multicolumn{1}{c}{ Treatment } & $\begin{array}{c}\text { Comprehensive Program } \\
\text { Evaluation }\end{array}$ \\
\hline D1 & Sensory Integration Treatment & NO \\
D2 & Vision Therapy & NO \\
D3 & Auditory Integration & NO \\
D4 & Art Therapy & NO \\
D5 & Music Therapy & NO \\
D6 & Facilitated Communication & NO \\
\hline
\end{tabular}

Table A2/5

Category E: Comprehensive Evaluation of Autism Medical Based Treatment

\begin{tabular}{clc}
\hline Category & \multicolumn{1}{c}{ Treatment } & $\begin{array}{c}\text { Comprehensive Program } \\
\text { Evaluation }\end{array}$ \\
\hline E1 & Amphetamines Treatments & NO \\
E2 & Antipsychotic-Benzodiazepines Treatment & NO \\
E3 & Antidepressants Treatment & NO
\end{tabular}


E4 Secretin Treatment NO

E5 Chelation Treatment NO

Table A2/6

Category F: Comprehensive Evaluation of Autism Biological Based Treatment

\begin{tabular}{clc}
\hline Category & Treatment & $\begin{array}{c}\text { Comprehensive Program } \\
\text { Evaluation }\end{array}$ \\
\hline F1 & Gluten- Free Treatment & NO \\
F2 & Casein Free Treatment & NO \\
F3 & Vitamin Therapy & NO \\
\hline
\end{tabular}


Table A2/7

Summary of Research Regarding Lovaas' DTT

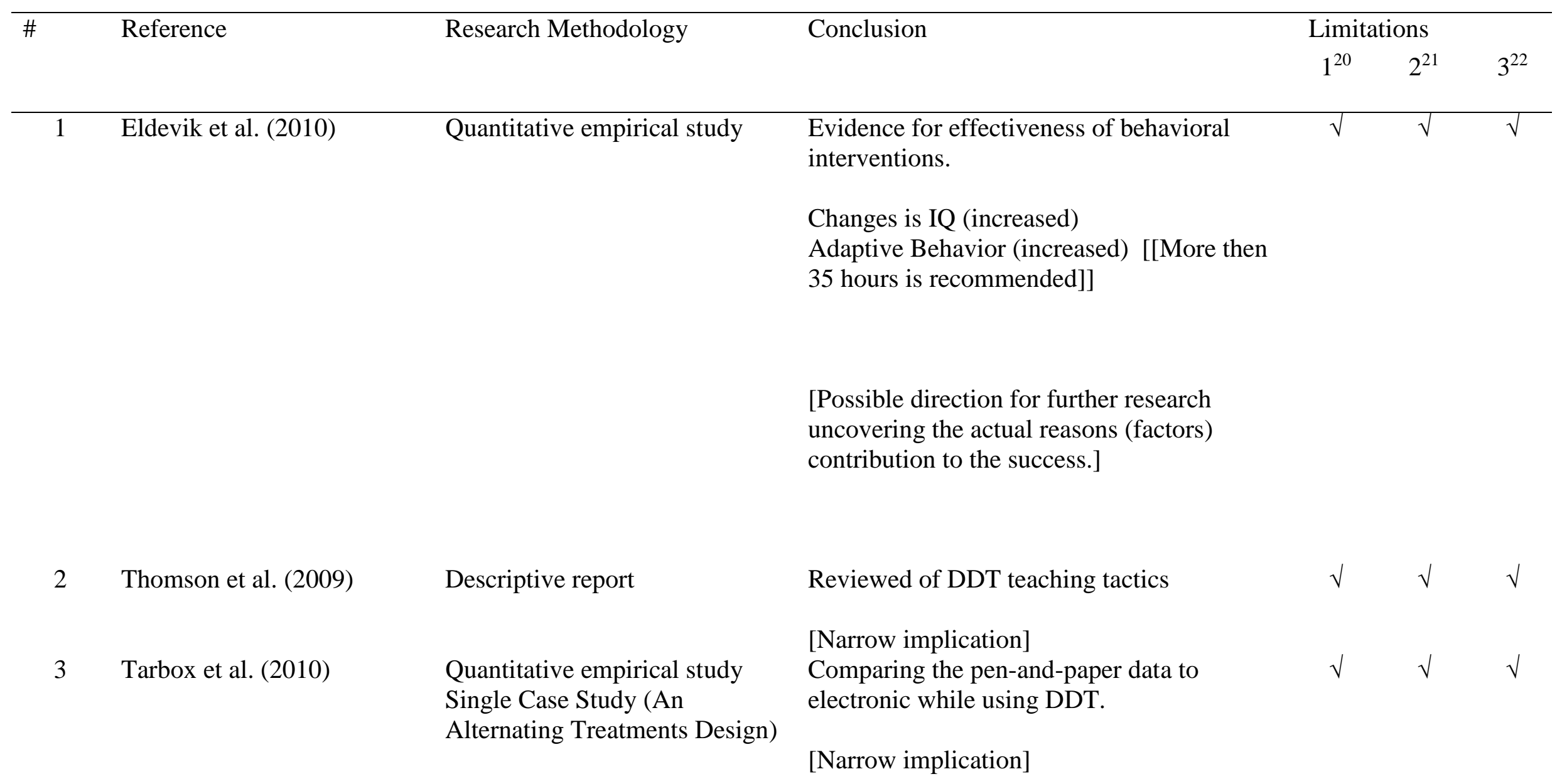

\footnotetext{
${ }^{20}$ Does not explain why or how the changes occurred

${ }^{21}$ Does not map the variety of symptoms spectrum (type and intensity)

${ }^{22}$ Does not conceptualize or address anything about Autism Complex Phenomenon per se and in what ways their research can inform the condition per se
} 
\#

Reference

Research Methodology

Conclusion

Limitations

$1^{20}$

$2^{21} \quad 3^{22}$

4

Gresham and MacMillan (1998)

Methodological criticism

Conceptualization of language training

Position Paper
Criticism of methodological faults in samplings, multiple threats to validity, and data analyses when Lovaas at all., claims that their intervention lead to recovery from Autism in $47 \%$ cases.

In addition authors recommend " healthy skepticism

It is recommended that parents and fair hearing officers adopt an attitude of healthy skepticism toward DDT.

[Methodological implication]

Practical guide for methodological training of specific language

[Narrow implication]

Comparison of financial benefit of DDT as oppose to special education

[Practical implication] 


\section{\#}

Reference Research Methodology

Research Methodology

Conclusion

Limitations

$1^{20} \quad 2^{21} \quad 3^{22}$

$7 \quad$ Thomson et al (2009)

Single Case

Training effectiveness of DDT instructors

and informing about the level accuracy of

prescribed performance and the need to

necessitate a certain criterion-level

performance to achieve optimal results when

serving to children with Autism.

[Narrow implication]

8 Gresham and MacMillan (1997)

Position Paper/ Reply to methodological issue

Conceptualization/ review article

Conceptualization criteria for Evidence based practices
Suggestion to admit methodological limitations (i.e., threats to external validity, generalizability) of Lovaas et al., empirical studies.

[Methodological implication]

Descriptive -informative about people with intellectual disability.

Authors review the psychological interventions that are evidence based.

They adopted Chambless and Hollon criteria of what is consider by Chambless \& Hoolon as (a) evidence based treatment and (b) effective treatment. The criteria are the followed: 
Single Case (Changing Criterion)
(1) Two randomized trails

(2) Trials have to be conducted by independent researcher

(3) Three small TV experimental studies with $(\mathrm{N}=9)$ participants

(4) TXs are "possibly effective" (according Chambless and Hoolon) if there are some studies indicated effectiveness and/or if the study was conducted only by one research team. Treatments are considered ineffective if they do not match the criteria.

[Preliminary conceptualization]

Evidence of using 10 consecutive discrete trails to increased students responding speed

[Narrow implication]

\section{Controversy about ABA}

\section{of ABA)}

Meta-analysis

Effect sizes measures (Hedges) yield to conclusion that early child intervention should be recommended. 


\begin{tabular}{|c|c|c|c|c|c|c|}
\hline \multirow[t]{2}{*}{ \# } & \multirow[t]{2}{*}{ Reference } & \multirow[t]{2}{*}{ Research Methodology } & \multirow[t]{2}{*}{ Conclusion } & \multicolumn{2}{|c|}{ Limitations } & \multirow[b]{2}{*}{$3^{22}$} \\
\hline & & & & $1^{20}$ & $2^{21}$ & \\
\hline & & & $\begin{array}{l}\text { [Positive changes in intelligence and } \\
\text { adaptive behavior] } \\
\text { [Possible direction for further research } \\
\text { uncovering the actual reasons (factors) } \\
\text { contribution to the success.] }\end{array}$ & & & \\
\hline \multirow[t]{3}{*}{14} & Warren et al (2011) & $\begin{array}{l}\text { Review of Literature regarding } \\
\text { evidence for early behavioral } \\
\text { interventions }\end{array}$ & $\begin{array}{l}\text { Authors reviewed } 34 \text { studies from which } \\
\text { they identified } 17 \text { were case series and } 2 \\
\text { were randomized controlled trails. }\end{array}$ & $\sqrt{ }$ & $\sqrt{ }$ & $\sqrt{ }$ \\
\hline & & & $\begin{array}{l}\text { Authors rated only } 1 \text { study as a good quality, } \\
10 \text { fair and } 23 \text { poor qualities. In sum authors } \\
\text { concluded that the strength of the evidence is } \\
\text { insufficient or low. }\end{array}$ & & & \\
\hline & & & $\begin{array}{l}\text { [Informative outcome }- \text { methodological and } \\
\text { conceptual implications] }\end{array}$ & & & \\
\hline \multirow[t]{2}{*}{15} & Pope, (1999) & Review/position paper & $\begin{array}{l}\text { Overall summation of history of Autism, } \\
\text { definition, etiology, diagnostic criteria, types } \\
\text { of Autism and Lovaas Early Intervention. }\end{array}$ & $\sqrt{ }$ & $\sqrt{ }$ & $\sqrt{ }$ \\
\hline & & & $\begin{array}{l}\text { The author suggests that if the intervention } \\
\text { will be implemented early, one-to-one } \\
\text { therapy for } 5-7 \text { hours and at least } 5 \text { days } \\
\text { (ideally } 7 \text { days) per week, then the individual } \\
\text { will achieve a normal functioning around } 7 \\
\text { years old. }\end{array}$ & & & \\
\hline
\end{tabular}


\#

Reference

Research Methodology

Conclusion

Limitations

$1^{20}$

$2^{2}$

The author supports her claim by Lovaas research.

[Informative/instructional outcome]

16 Jonson (1977)
Review of the book:

"Handbook of Behavior

Modification and Behavior

Therapy"

Single Case Design
Jonson suggests that the author of the book is praising (kind of "bible" style) the behaviorists' contributions regarding behavioral modification and/or therapy.

According to Johnson the book could be used as a textbook for specialized graduate courses.

[Practical implication]

Authors study the performance training of 12 DDT teaching responses.

Employ the Single Case Design to conclude that there is a need to ensure that instructors of DTT will achieve a certain criterion level of performance. 


\begin{tabular}{|c|c|c|c|c|c|}
\hline & \multirow[t]{2}{*}{ Reference } & \multirow[t]{2}{*}{ Research Methodology } & \multirow[t]{2}{*}{ Conclusion } & \multicolumn{2}{|l|}{ Limitations } \\
\hline & & & & $1^{20} \quad 2^{21}$ & $3^{22}$ \\
\hline
\end{tabular}

[Narrow outcome and by design and a topic

very similar to Fienup and Doepke, (2008)]

Author review common knowledge about Autism characteristics and treatments.

Author suggests that behavioral interventions such as Lovaas DDT lead to positive results.

Employment of the (pre and post treatment) quasi-experimental design led to statistically significant result suggesting that Puentes program is an effective intervention.

[Possible direction for further research uncovering the actual reasons (factors) contribution to the success.]

19 Park and National Society for Autistic Children (1971)

Conference Report (position paper/speeches)
Single Case (multiple-baseline design across subjects)
The report summarized the speeches regarding Federal reaction toward disabled children; Lovaas' speech evaluating pros and cons of treatments for Autism based on the operant condition tactics; and the research study regarding vitamins treatment.

[Practical implication for the conference attendees]

The author evaluated the effectiveness of the Intensive Early Intervention. He assessed 
\#

Reference

Research Methodology

Conclusion

Limitations

$1^{20}$

$2^{21} \quad 3^{22}$

students' attainment of 40 different tasks and the optimal delivery. Author concluded that students acquired the skills slower then comparable group. And that the parents and paraprofessional would be optimal for delivery of DDT.

[Narrow implication]

$21 \quad$ Lovaas (1987)
Quantitative - empirical study

Quantitative- follow up study
The experimental group received DDT and resulting in a statistically significant increase in IQ in comparison with control group.

[Possible direction for further research uncovering the actual reasons (factors) contribution to the success.]

Confirmed Lovaas successes from 1987 by followed up study. The followed up study find that 8 children from the experimental group (intensive DTT) have average IQ and adaptive behavior. 


\section{\#}

Reference

Research Methodology

Conclusion

Limitations

$1^{20}$

Indeed, we do not know if these children have had any other treatments and or interventions.

[Possible direction for further research uncovering the actual reasons (factors) contribution to the success.]

The experimental group obtained 30 hours per week of DDT (Lovaas suggested 40) indicated statistically significant increase in IQ, academic skills, language development and not a significant difference in adaptive behavior when comparing to control group.

[Possible direction for further research uncovering the actual reasons (factors) contribution to the success.] 
Table A2/8

Summary of Research Regarding: Pivotal Response Training

\begin{tabular}{|c|c|c|c|c|c|c|}
\hline Reference & $\begin{array}{c}\text { Research } \\
\text { Methodology }\end{array}$ & Authors' Conclusions & $\begin{array}{c}\text { Is it a } \\
\text { Comprehensive } \\
\text { Evaluation of } \\
\text { an Entire } \\
\text { Program? }\end{array}$ & $\begin{array}{l}\text { Does it } \\
\text { Demonstrate } \\
\text { How Each of } \\
\text { the Program } \\
\text { Components } \\
\text { Produce the } \\
\text { Changes? }\end{array}$ & $\begin{array}{l}\text { Does it Provide } \\
\text { Detailed } \\
\text { Descriptions of } \\
\text { the Symptoms } \\
\text { for Each } \\
\text { Participant? }\end{array}$ & $\begin{array}{c}\text { Does it Connect } \\
\text { the Results of the } \\
\text { Investigation to } \\
\text { Our } \\
\text { Understanding of } \\
\text { Autism as a } \\
\text { Complex } \\
\text { Phenomenon? }\end{array}$ \\
\hline $\begin{array}{l}\text { Hayes } \\
(2009)\end{array}$ & Qualitative & $\begin{array}{l}\text { The findings of (single) interview } \\
\text { indicate that early intervention is } \\
\text { necessary for children with Asperger. In } \\
\text { addition to, the interviewee (Lynn } \\
\text { Koegel) differentiate between a person } \\
\text { with Autism (language delay, impaired } \\
\text { social communication, limited interest) } \\
\text { and person with Asperger's (problems } \\
\text { with social and limited interest but not } \\
\text { with language development) }\end{array}$ & $\mathrm{NO}$ & $\mathrm{NO}$ & NO & $\mathrm{NO}$ \\
\hline $\begin{array}{l}\text { Coolican et } \\
\text { al. (2010) }\end{array}$ & $\begin{array}{l}\text { Single-Case } \\
\text { Experimental } \\
\text { Design }\end{array}$ & $\begin{array}{l}\text { Assessment of a brief training in PRT for } \\
\text { parents who (a) has a child with Autism } \\
\text { and that child is pre-schooled age and (b) } \\
\text { the child is not receiving treatment. The } \\
\text { study suggests that in general the } \\
\text { children skills increased in } \\
\text { communication skills. }\end{array}$ & NO & NO & NO & $\mathrm{NO}$ \\
\hline
\end{tabular}




\begin{tabular}{|c|c|c|c|c|c|c|}
\hline Reference & $\begin{array}{c}\text { Research } \\
\text { Methodology }\end{array}$ & Authors' Conclusions & $\begin{array}{c}\text { Is it a } \\
\text { Comprehensive } \\
\text { Evaluation of } \\
\text { an Entire } \\
\text { Program? }\end{array}$ & $\begin{array}{l}\text { Does it } \\
\text { Demonstrate } \\
\text { How Each of } \\
\text { the Program } \\
\text { Components } \\
\text { Produce the } \\
\text { Changes? }\end{array}$ & $\begin{array}{l}\text { Does it Provide } \\
\text { Detailed } \\
\text { Descriptions of } \\
\text { the Symptoms } \\
\text { for Each } \\
\text { Participant? }\end{array}$ & $\begin{array}{c}\text { Does it Connect } \\
\text { the Results of the } \\
\text { Investigation to } \\
\text { Our } \\
\text { Understanding of } \\
\text { Autism as a } \\
\text { Complex } \\
\text { Phenomenon? }\end{array}$ \\
\hline
\end{tabular}

Authors suggested that a brief training for parents might provide some intervention while it is cost-effective.

Smith et al. Quantitative Due to employment of Pivotal Response (2010)

$(\mathrm{N}=45)$ children with Autism

significantly decreed their behavioral problems. However, the Autism

symptoms decreased only if the participants had IQ 50 and above.

Koegel et al. Single-Case The study assesses three parts of self-

(2013) Experimental management intervention. Researchers

Design

suggested that the intervention led to

"meaningful improvements" in conversation.

Koegel et al. Single-Case Assessment of a single intervention

(2013) Experimental

Design

(Structured Social Planning) for college students with ASD.

Participants increased their social interaction by increasing the number of participation of the social events.

Koegel et al. Single-Case (2013)

Experimental Design
Authors assess the efficacy of using

motivational procedures of the Pivotal response Training. Authors concluded
NO

NO

Basic

description of

the participants

demographics

NO

NO

Some

description

NO

NO

NO

NO

NO

NO

NO

NO

NO 


\begin{tabular}{|c|c|c|c|c|c|c|}
\hline Reference & $\begin{array}{c}\text { Research } \\
\text { Methodology }\end{array}$ & Authors' Conclusions & $\begin{array}{l}\text { Is it a } \\
\text { Comprehensive } \\
\text { Evaluation of } \\
\text { an Entire } \\
\text { Program? }\end{array}$ & $\begin{array}{l}\text { Does it } \\
\text { Demonstrate } \\
\text { How Each of } \\
\text { the Program } \\
\text { Components } \\
\text { Produce the } \\
\text { Changes? }\end{array}$ & $\begin{array}{c}\text { Does it Provide } \\
\text { Detailed } \\
\text { Descriptions of } \\
\text { the Symptoms } \\
\text { for Each } \\
\text { Participant? }\end{array}$ & $\begin{array}{l}\text { Does it Connect } \\
\text { the Results of the } \\
\text { Investigation to } \\
\text { Our } \\
\text { Understanding of } \\
\text { Autism as a } \\
\text { Complex } \\
\text { Phenomenon? }\end{array}$ \\
\hline $\begin{array}{l}\text { Koegel et al. } \\
(2013)\end{array}$ & $\begin{array}{l}\text { Single-Case } \\
\text { Experimental } \\
\text { Design }\end{array}$ & $\begin{array}{l}\text { that after the intervention the participants } \\
\text { increased social interaction. } \\
\text { Assessment of lunchtime activates to } \\
\text { increase engagement of the high school } \\
\text { students with Autism. Positive results }\end{array}$ & NO & NO & NO & $\mathrm{NO}$ \\
\hline $\begin{array}{l}\text { Vernon et } \\
\text { al. (2012) }\end{array}$ & $\begin{array}{l}\text { Single-Case } \\
\text { Experimental } \\
\text { Design }\end{array}$ & $\begin{array}{l}\text { Assessment of parents' social } \\
\text { engagement with their children. The } \\
\text { intervention led to increasing eye contact, } \\
\text { verbal initiation, and parents' positive } \\
\text { effect. }\end{array}$ & $\mathrm{NO}$ & $\mathrm{NO}$ & NO & $\mathrm{NO}$ \\
\hline $\begin{array}{l}\text { Koegel et al. } \\
(2012)\end{array}$ & $\begin{array}{l}\text { Single-Case } \\
\text { Experimental } \\
\text { Design }\end{array}$ & $\begin{array}{l}\text { Assessment of individualized } \\
\text { reinforcement and hierarchical exposure } \\
\text { to increase flexibility. Results indicate } \\
\text { increasing behavior in terms of } \\
\text { requesting new foods. }\end{array}$ & $\mathrm{NO}$ & $\mathrm{NO}$ & NO & $\mathrm{NO}$ \\
\hline $\begin{array}{l}\text { Koegel et al. } \\
(2010)\end{array}$ & $\begin{array}{l}\text { Single-Case } \\
\text { Experimental } \\
\text { Design }\end{array}$ & $\begin{array}{l}\text { Assessment of choice, interspersal of } \\
\text { maintenance tasks, and natural reinforces } \\
\text { improve performance and interest. } \\
\text { Students decreased the latency to begin } \\
\text { academic tasks, improved their rate of } \\
\text { performance and interest, and decreased } \\
\text { their disruptive behavior. }\end{array}$ & NO & $\mathrm{NO}$ & NO & $\begin{array}{c}\text { NO } \\
\text { But some } \\
\text { Theoretical and } \\
\text { applied } \\
\text { suggestions were } \\
\text { discussed }\end{array}$ \\
\hline $\begin{array}{l}\text { Bryson et al. } \\
\text { (2007) }\end{array}$ & $\begin{array}{l}\text { Position } \\
\text { Paper }\end{array}$ & $\begin{array}{l}\text { Descriptive paper about PRT in Nova } \\
\text { Scotia and Canada. }\end{array}$ & NO & NO & NO & $\mathrm{NO}$ \\
\hline
\end{tabular}




\begin{tabular}{|c|c|c|c|c|c|c|}
\hline Reference & $\begin{array}{c}\text { Research } \\
\text { Methodology }\end{array}$ & Authors' Conclusions & $\begin{array}{c}\text { Is it a } \\
\text { Comprehensive } \\
\text { Evaluation of } \\
\text { an Entire } \\
\text { Program? }\end{array}$ & $\begin{array}{c}\text { Does it } \\
\text { Demonstrate } \\
\text { How Each of } \\
\text { the Program } \\
\text { Components } \\
\text { Produce the } \\
\text { Changes? }\end{array}$ & $\begin{array}{l}\text { Does it Provide } \\
\text { Detailed } \\
\text { Descriptions of } \\
\text { the Symptoms } \\
\text { for Each } \\
\text { Participant? }\end{array}$ & $\begin{array}{c}\text { Does it Connect } \\
\text { the Results of the } \\
\text { Investigation to } \\
\text { Our } \\
\text { Understanding of } \\
\text { Autism as a } \\
\text { Complex } \\
\text { Phenomenon? }\end{array}$ \\
\hline
\end{tabular}

Satisfaction data (survey/descriptive statistics) on stakeholders' satisfaction.

Koegel et al Single-Case Assessment of "Where" questions" via (2010) Experimental use of intrinsic reinforcement to create Design the generalized use of the questions.

Results indicate that children could acquire and generalize the inquiry

Koegel et al. Single-Case Assessment of (a) individualized (2009) Experimental Design

orienting cues identification and (b) if

their presentation result in expressive words.

Results indicate increase correct responding to verbal representations as well as subsequent word use.

But some Theoretical and applied implications of this intervention are discussed

NO NO NO NO (2009)

Experimental
Design

Assessment of embedding social interactions would lead to increased levels of child-initiated social behaviors.
But some

Theoretical and applied 


\begin{tabular}{|c|c|c|c|c|c|c|}
\hline Reference & $\begin{array}{c}\text { Research } \\
\text { Methodology }\end{array}$ & Authors' Conclusions & $\begin{array}{l}\text { Is it a } \\
\text { Comprehensive } \\
\text { Evaluation of } \\
\text { an Entire } \\
\text { Program? }\end{array}$ & $\begin{array}{l}\text { Does it } \\
\text { Demonstrate } \\
\text { How Each of } \\
\text { the Program } \\
\text { Components } \\
\text { Produce the } \\
\text { Changes? }\end{array}$ & $\begin{array}{l}\text { Does it Provide } \\
\text { Detailed } \\
\text { Descriptions of } \\
\text { the Symptoms } \\
\text { for Each } \\
\text { Participant? }\end{array}$ & $\begin{array}{c}\text { Does it Connect } \\
\text { the Results of the } \\
\text { Investigation to } \\
\text { Our } \\
\text { Understanding of } \\
\text { Autism as a } \\
\text { Complex } \\
\text { Phenomenon? }\end{array}$ \\
\hline & & $\begin{array}{l}\text { Results indicate increases in child- } \\
\text { initiated social engagement during } \\
\text { communication, improved nonverbal } \\
\text { dyadic orienting, and improvements in } \\
\text { general child affect. }\end{array}$ & & & & $\begin{array}{l}\text { implications are } \\
\text { discussed }\end{array}$ \\
\hline $\begin{array}{l}\text { Koegel et al. } \\
(2005)\end{array}$ & $\begin{array}{l}\text { Conceptual/ } \\
\text { theoretical } \\
\text { Paper }\end{array}$ & $\begin{array}{l}\text { Description of a early identification } \\
\text { model for children with autism spectrum }\end{array}$ & NO & NO & NO & $\begin{array}{l}\text { Theoretical } \\
\text { explanation for } \\
\text { the need to early } \\
\text { identify Autism }\end{array}$ \\
\hline $\begin{array}{l}\text { Koegel at al. } \\
(2012)\end{array}$ & $\begin{array}{l}\text { Single-Case } \\
\text { Experimental } \\
\text { Design }\end{array}$ & $\begin{array}{l}\text { Assessment of generalization in the } \\
\text { absence of interventionists in elementary } \\
\text { school children with ASD at recess. }\end{array}$ & NO & NO & NO & NO \\
\hline & & $\begin{array}{l}\text { Results indicate that students } \\
\text { demonstrated generalized peer social } \\
\text { engagement, increases in unprompted } \\
\text { peer-directed initiations, and positive } \\
\text { affect during peer interactions. }\end{array}$ & & & & \\
\hline $\begin{array}{l}\text { Ventola } \\
\text { (2013) }\end{array}$ & Book Review & $\begin{array}{l}\text { Review of "The PRT Pocket Guide: } \\
\text { Pivotal Response Treatment for } \\
\text { Autism Spectrum Disorders" }\end{array}$ & NO & NO & NO & NO \\
\hline $\begin{array}{l}\text { Voress } \\
(2005)\end{array}$ & $\begin{array}{l}\text { Position } \\
\text { Paper }\end{array}$ & Opinion & NO & NO & NO & NO \\
\hline
\end{tabular}




\begin{tabular}{|c|c|c|c|c|c|c|}
\hline Reference & $\begin{array}{c}\text { Research } \\
\text { Methodology }\end{array}$ & Authors' Conclusions & $\begin{array}{c}\text { Is it a } \\
\text { Comprehensive } \\
\text { Evaluation of } \\
\text { an Entire } \\
\text { Program? }\end{array}$ & $\begin{array}{c}\text { Does it } \\
\text { Demonstrate } \\
\text { How Each of } \\
\text { the Program } \\
\text { Components } \\
\text { Produce the } \\
\text { Changes? }\end{array}$ & $\begin{array}{l}\text { Does it Provide } \\
\text { Detailed } \\
\text { Descriptions of } \\
\text { the Symptoms } \\
\text { for Each } \\
\text { Participant? }\end{array}$ & $\begin{array}{l}\text { Does it Connect } \\
\text { the Results of the } \\
\text { Investigation to } \\
\text { Our } \\
\text { Understanding of } \\
\text { Autism as a } \\
\text { Complex } \\
\text { Phenomenon? }\end{array}$ \\
\hline $\begin{array}{l}\text { Koegel et al. } \\
(2001)\end{array}$ & $\begin{array}{l}\text { Single-Case } \\
\text { Experimental } \\
\text { Design }\end{array}$ & $\begin{array}{l}\text { The study identified the need for } \\
\text { intervention for children with Autism in } \\
\text { the area of social skills. }\end{array}$ & $\mathrm{NO}$ & $\mathrm{NO}$ & NO & $\mathrm{NO}$ \\
\hline $\begin{array}{l}\text { Koegel et al. } \\
(2002)\end{array}$ & $\begin{array}{l}\text { Single-Case } \\
\text { Experimental } \\
\text { Design }\end{array}$ & $\begin{array}{l}\text { Study assessed a weeklong education } \\
\text { program (Pivotal Response Training) for } \\
\text { parents so that they can improve social } \\
\text { communication for children with autism. } \\
\text { Researchers suggest that logistics } \\
\text { difficulty can be overcome by intensive } \\
\text { parent education program. }\end{array}$ & NO & NO & NO & NO \\
\hline $\begin{array}{l}\text { Koegel et al. } \\
\text { (1998) }\end{array}$ & $\begin{array}{l}\text { Position } \\
\text { Paper }\end{array}$ & $\begin{array}{l}\text { Discussion about maximizing effect of } \\
\text { treatment for children with Autism }\end{array}$ & NO & NO & NO & NO \\
\hline $\begin{array}{l}\text { Koegel et al. } \\
\text { (1997) }\end{array}$ & $\begin{array}{l}\text { Single-Case } \\
\text { Experimental } \\
\text { Design }\end{array}$ & $\begin{array}{l}\text { Researchers assessed and suggested to } \\
\text { improve motivation and attention to } \\
\text { positively influence students with Autism } \\
\text { performance on standardized test }\end{array}$ & NO & NO & NO & NO \\
\hline $\begin{array}{l}\text { Koegel et al. } \\
\text { (1992) }\end{array}$ & $\begin{array}{l}\text { Single-Case } \\
\text { Experimental } \\
\text { Design }\end{array}$ & $\begin{array}{l}\text { Researchers assessed and suggested that } \\
\text { children with Autism can learn self- } \\
\text { management to respond others and } \\
\text { reduction in disruptive behavior. }\end{array}$ & NO & NO & NO & NO \\
\hline
\end{tabular}




\begin{tabular}{|c|c|c|c|c|c|c|}
\hline Reference & $\begin{array}{c}\text { Research } \\
\text { Methodology }\end{array}$ & Authors' Conclusions & $\begin{array}{l}\text { Is it a } \\
\text { Comprehensive } \\
\text { Evaluation of } \\
\text { an Entire } \\
\text { Program? }\end{array}$ & $\begin{array}{c}\text { Does it } \\
\text { Demonstrate } \\
\text { How Each of } \\
\text { the Program } \\
\text { Components } \\
\text { Produce the } \\
\text { Changes? }\end{array}$ & $\begin{array}{l}\text { Does it Provide } \\
\text { Detailed } \\
\text { Descriptions of } \\
\text { the Symptoms } \\
\text { for Each } \\
\text { Participant? }\end{array}$ & $\begin{array}{c}\text { Does it Connect } \\
\text { the Results of the } \\
\text { Investigation to } \\
\text { Our } \\
\text { Understanding of } \\
\text { Autism as a } \\
\text { Complex } \\
\text { Phenomenon? }\end{array}$ \\
\hline $\begin{array}{l}\text { Brookman } \\
\text { et al. }(2003)\end{array}$ & $\begin{array}{l}\text { Descriptive } \\
\text { Paper }\end{array}$ & $\begin{array}{l}\text { Implementation and description of } \\
\text { summer program for children with } \\
\text { Autism to increase participation. }\end{array}$ & $\mathrm{NO}$ & NO & $\mathrm{NO}$ & NO \\
\hline $\begin{array}{l}\text { Koegel et al. } \\
(2003)\end{array}$ & $\begin{array}{l}\text { Single-Case } \\
\text { Experimental } \\
\text { Design }\end{array}$ & $\begin{array}{l}\text { Researchers assessed and suggested, } \\
\text { "priming" to decrease problem behavior } \\
\text { and increase academic performance. }\end{array}$ & NO & NO & NO & NO \\
\hline $\begin{array}{l}\text { Koegel et al. } \\
\text { (1988) }\end{array}$ & $\begin{array}{l}\text { Single-Case } \\
\text { Experimental } \\
\text { Design }\end{array}$ & $\begin{array}{l}\text { Researchers assessed and suggested that } \\
\text { for improvement in students with Autism } \\
\text { speech the reinforcing speech is more } \\
\text { effective than reinforcing motor speech } \\
\text { sound. }\end{array}$ & NO & NO & NO & NO \\
\hline $\begin{array}{l}\text { Wilczynski } \\
\text { et al. (2011) }\end{array}$ & Book & $\begin{array}{l}\text { Chapter overview of ASD, treatments, } \\
\text { diagnostics process, and identification of } \\
\text { the most effective interventions. }\end{array}$ & NO & NO & $\begin{array}{c}\text { General } \\
\text { Overview }\end{array}$ & NO \\
\hline $\begin{array}{l}\text { Koegel et al. } \\
(2009)\end{array}$ & Book & $\begin{array}{l}\text { Guide for parents to effectively address } \\
\text { multiple questions and issues regarding } \\
\text { their children with Autism; description of } \\
\text { Koegel Autism Research and Training } \\
\text { Center. }\end{array}$ & NO & NO & NO & $\mathrm{NO}$ \\
\hline
\end{tabular}




\begin{tabular}{|c|c|c|c|c|c|c|}
\hline Reference & $\begin{array}{c}\text { Research } \\
\text { Methodology }\end{array}$ & Authors' Conclusions & $\begin{array}{c}\text { Is it a } \\
\text { Comprehensive } \\
\text { Evaluation of } \\
\text { an Entire } \\
\text { Program? }\end{array}$ & $\begin{array}{l}\text { Does it } \\
\text { Demonstrate } \\
\text { How Each of } \\
\text { the Program } \\
\text { Components } \\
\text { Produce the } \\
\text { Changes? }\end{array}$ & $\begin{array}{l}\text { Does it Provide } \\
\text { Detailed } \\
\text { Descriptions of } \\
\text { the Symptoms } \\
\text { for Each } \\
\text { Participant? }\end{array}$ & $\begin{array}{c}\text { Does it Connect } \\
\text { the Results of the } \\
\text { Investigation to } \\
\text { Our } \\
\text { Understanding of } \\
\text { Autism as a } \\
\text { Complex } \\
\text { Phenomenon? }\end{array}$ \\
\hline $\begin{array}{l}\text { Koegel et al. } \\
(2010)\end{array}$ & Book & $\begin{array}{l}\text { Chapter regarding explanation and } \\
\text { description of pivotal motivation in } \\
\text { children with Autism and identification } \\
\text { of other pivotal responses. }\end{array}$ & NO & NO & $\mathrm{NO}$ & $\mathrm{NO}$ \\
\hline $\begin{array}{l}\text { Koegel et al. } \\
(2006)\end{array}$ & Book & $\begin{array}{l}\text { Description of Pivotal Response } \\
\text { Treatment and the need to keep parents } \\
\text { involved closely in their children } \\
\text { treatment. }\end{array}$ & NO & NO & NO & NO \\
\hline & & $\begin{array}{l}\text { Book suggest that research suggest that } \\
\text { PRT lead to: } \\
\text { (a) Improvement children's academic } \\
\text { performance } \\
\text { (b) Enhancement of children's } \\
\text { communication and language skills } \\
\text { (c) Promotion of social interactions } \\
\text { (d) Reduction of disruptive behaviors } \\
\text { (e) Assistance in early identification } \\
\text { (f) Reduction of ritualistic behaviors } \\
\text { (g) Expanding of children's interests }\end{array}$ & & & & \\
\hline $\begin{array}{l}\text { Koegel et al. } \\
(2008)\end{array}$ & Book & $\begin{array}{l}\text { Book chapter: Summary of available } \\
\text { treatments and suggestion for delivery } \\
\text { for ASD }\end{array}$ & NO & NO & $\mathrm{NO}$ & NO \\
\hline
\end{tabular}




\begin{tabular}{|c|c|c|c|c|c|c|}
\hline Reference & $\begin{array}{c}\text { Research } \\
\text { Methodology }\end{array}$ & Authors' Conclusions & $\begin{array}{l}\text { Is it a } \\
\text { Comprehensive } \\
\text { Evaluation of } \\
\text { an Entire } \\
\text { Program? }\end{array}$ & $\begin{array}{l}\text { Does it } \\
\text { Demonstrate } \\
\text { How Each of } \\
\text { the Program } \\
\text { Components } \\
\text { Produce the } \\
\text { Changes? }\end{array}$ & $\begin{array}{l}\text { Does it Provide } \\
\text { Detailed } \\
\text { Descriptions of } \\
\text { the Symptoms } \\
\text { for Each } \\
\text { Participant? }\end{array}$ & $\begin{array}{c}\text { Does it Connect } \\
\text { the Results of the } \\
\text { Investigation to } \\
\text { Our } \\
\text { Understanding of } \\
\text { Autism as a } \\
\text { Complex } \\
\text { Phenomenon? }\end{array}$ \\
\hline $\begin{array}{l}\text { Koegel et al. } \\
(2005)\end{array}$ & Book & $\begin{array}{l}\text { Description and identification of the data } \\
\text { based treatments for children with } \\
\text { Autism. Presentation of variety of } \\
\text { techniques to enhance variety of } \\
\text { behavior. }\end{array}$ & NO & NO & NO & NO \\
\hline $\begin{array}{l}\text { Schreibman } \\
\text { et al. (2005) }\end{array}$ & Book Review & $\begin{array}{l}\text { Summary of getting parents } \\
\text { systematically involved into treatments } \\
\text { for their children. }\end{array}$ & NO & NO & $\mathrm{NO}$ & NO \\
\hline \multirow[t]{2}{*}{$\begin{array}{l}\text { Koegel et al. } \\
\text { (1995) }\end{array}$} & Book & $\begin{array}{l}\text { Book chapter: Summarizes the } \\
\text { importance of self-management and } \\
\text { imply the need to develop such type of } \\
\text { interventions program that would lead to } \\
\text { multiple gains. }\end{array}$ & NO & NO & NO & NO \\
\hline & & $\begin{array}{l}\text { Authors also speculate that the more the } \\
\text { persons interact with environment there } \\
\text { is larger possibility to enhance } \\
\text { neurological development. }\end{array}$ & & & & \\
\hline
\end{tabular}




\begin{tabular}{|c|c|c|c|c|c|c|}
\hline Reference & $\begin{array}{c}\text { Research } \\
\text { Methodology }\end{array}$ & Authors' Conclusions & $\begin{array}{l}\text { Is it a } \\
\text { Comprehensive } \\
\text { Evaluation of } \\
\text { an Entire } \\
\text { Program? }\end{array}$ & $\begin{array}{l}\text { Does it } \\
\text { Demonstrate } \\
\text { How Each of } \\
\text { the Program } \\
\text { Components } \\
\text { Produce the } \\
\text { Changes? }\end{array}$ & $\begin{array}{l}\text { Does it Provide } \\
\text { Detailed } \\
\text { Descriptions of } \\
\text { the Symptoms } \\
\text { for Each } \\
\text { Participant? }\end{array}$ & $\begin{array}{l}\text { Does it Connect } \\
\text { the Results of the } \\
\text { Investigation to } \\
\text { Our } \\
\text { Understanding of } \\
\text { Autism as a } \\
\text { Complex } \\
\text { Phenomenon? }\end{array}$ \\
\hline $\begin{array}{l}\text { Koegel et al. } \\
(1995)\end{array}$ & Book Review & $\begin{array}{l}\text { Description of the most common } \\
\text { characteristics of Autism, behavioral } \\
\text { diagnostics, and interventions for } \\
\text { children wit Autism. }\end{array}$ & NO & NO & $\begin{array}{l}\text { General } \\
\text { Description of } \\
\text { Symptoms }\end{array}$ & $\mathrm{NO}$ \\
\hline $\begin{array}{l}\text { Koegel et al. } \\
(1995)\end{array}$ & Book & $\begin{array}{l}\text { A guide and particular procedures for } \\
\text { parents and teachers to effectively teach } \\
\text { children with Autism. }\end{array}$ & NO & NO & NO & NO \\
\hline & & $\begin{array}{l}\text { Discussion about the vide variety of } \\
\text { different manifestation of Autism. }\end{array}$ & & & & \\
\hline $\begin{array}{l}\text { Rosenblatt } \\
\text { ET AL. } \\
(1995)\end{array}$ & Book & $\begin{array}{l}\text { Book Chapter: The need to provide } \\
\text { intervention for children with Autism; } \\
\text { interventions that produce generalized } \\
\text { responding can achieve multiple } \\
\text { outcomes. }\end{array}$ & NO & NO & NO & NO \\
\hline $\begin{array}{l}\text { Sherer } \\
(2003)\end{array}$ & $\begin{array}{l}\text { Dissertation } \\
\text { Single-Case } \\
\text { Experimental } \\
\text { Design }\end{array}$ & $\begin{array}{l}\text { Authors propose that about } 50 \% \text { of } \\
\text { children with Autism significantly } \\
\text { benefit from the treatment but } 50 \% \text { do } \\
\text { not benefit from it. (Differential } \\
\text { Responsiveness) }\end{array}$ & NO & NO & $\begin{array}{l}\text { Develops } \\
\text { several } \\
\text { behavioral } \\
\text { profiles } \\
\text { based on } \\
\text { symptoms }\end{array}$ & NO \\
\hline
\end{tabular}




\begin{tabular}{|c|c|c|c|c|c|c|}
\hline Reference & $\begin{array}{c}\text { Research } \\
\text { Methodology }\end{array}$ & Authors' Conclusions & $\begin{array}{c}\text { Is it a } \\
\text { Comprehensive } \\
\text { Evaluation of } \\
\text { an Entire } \\
\text { Program? }\end{array}$ & $\begin{array}{l}\text { Does it } \\
\text { Demonstrate } \\
\text { How Each of } \\
\text { the Program } \\
\text { Components } \\
\text { Produce the } \\
\text { Changes? }\end{array}$ & $\begin{array}{l}\text { Does it Provide } \\
\text { Detailed } \\
\text { Descriptions of } \\
\text { the Symptoms } \\
\text { for Each } \\
\text { Participant? }\end{array}$ & $\begin{array}{l}\text { Does it Connect } \\
\text { the Results of the } \\
\text { Investigation to } \\
\text { Our } \\
\text { Understanding of } \\
\text { Autism as a } \\
\text { Complex } \\
\text { Phenomenon? }\end{array}$ \\
\hline & & $\begin{array}{l}\text { Author speculates that the differential } \\
\text { responsiveness suggests that all persons } \\
\text { with Autism cannot benefit from one } \\
\text { single intervention. Therefore, author } \\
\text { proposes to identify the person's } \\
\text { characteristics by creating behavioral } \\
\text { profile that are connected with different } \\
\text { outcomes for a specific intervention. }\end{array}$ & & & & \\
\hline $\begin{array}{l}\text { Lozowski- } \\
\text { Sullivan } \\
(2012)\end{array}$ & Dissertation & $\begin{array}{l}\text { Author questioned the reliability and } \\
\text { validity of commonly used measurement } \\
\text { for Autism and suggests using multiple- } \\
\text { multiple trait assessment. }\end{array}$ & NO & NO & NO & NO \\
\hline
\end{tabular}


Table A2/9

Summary of Research Regarding: The Strategic Teaching and Reinforcement System: Verbal Behavior program

\begin{tabular}{|c|c|c|c|c|c|c|}
\hline Reference & $\begin{array}{c}\text { Research } \\
\text { Methodology }\end{array}$ & Authors' Conclusions & $\begin{array}{c}\text { Is it a } \\
\text { Comprehensive } \\
\text { Evaluation of } \\
\text { an Entire } \\
\text { Program? }\end{array}$ & $\begin{array}{l}\text { Does it } \\
\text { Demonstrate } \\
\text { How Each of } \\
\text { the Program } \\
\text { Components } \\
\text { Produce the } \\
\text { Changes? }\end{array}$ & $\begin{array}{l}\text { Does it Provide } \\
\text { Detailed } \\
\text { Descriptions of } \\
\text { the Symptoms } \\
\text { for Each } \\
\text { Participant? }\end{array}$ & $\begin{array}{l}\text { Does it Connect } \\
\text { the Results of the } \\
\text { Investigation to } \\
\text { Our } \\
\text { Understanding of } \\
\text { Autism as a } \\
\text { Complex } \\
\text { Phenomenon? }\end{array}$ \\
\hline $\begin{array}{l}\text { Carr and } \\
\text { Firth }(2005)\end{array}$ & $\begin{array}{l}\text { Conceptual } \\
\text { paper }\end{array}$ & $\begin{array}{l}\text { Conceptualizing paper that point out (a) } \\
\text { that the verbal-behavior approach is not } \\
\text { empirically well supported (while } \\
\text { conceptually sounded) and (b) offers the } \\
\text { way how to collect and publish data for } \\
\text { practitioners. }\end{array}$ & NO & $\mathrm{NO}$ & NO & NO \\
\hline $\begin{array}{l}\text { Young } \\
(2007)\end{array}$ & Dissertations & $\begin{array}{l}\text { Behaviorally based Star Program lead to } \\
\text { significantly improvement more } \\
\text { expressive language, receptive language, } \\
\text { social interaction, and pre-academic } \\
\text { skills; and significantly fewer stereotypic } \\
\text { behaviors. }\end{array}$ & $\mathrm{NO}$ & NO & NO & $\mathrm{NO}$ \\
\hline $\begin{array}{l}\text { Weiss and } \\
\text { Demiri } \\
(2011)\end{array}$ & Book & $\begin{array}{l}\text { Address/describe the Applied Verbal } \\
\text { Behavior method and the need to } \\
\text { incorporate development of language } \\
\text { early in Autism treatment. The book also } \\
\text { described other approaches such as: sign } \\
\text { language, video modeling, scripts, and } \\
\text { Social Stories. The objective of the book } \\
\text { is to provide parents guide and } \\
\text { suggestion to improve communication } \\
\text { skills in their children. }\end{array}$ & NO & $\mathrm{NO}$ & NO & NO \\
\hline
\end{tabular}


Table A2/10

Glossary of New Terms

\begin{tabular}{ll}
\hline \multicolumn{1}{c}{ New Term } & \multicolumn{1}{c}{ Explanation } \\
\hline The Incidence Autism Rate & $\begin{array}{l}\text { The rate of Autism where new cases are counted per person- } \\
\text { year }\end{array}$ \\
The Cumulative Autism Incidence & $\begin{array}{l}\text { In a specified time period the total number of new Autism } \\
\text { cases }\end{array}$ \\
The Point Autism Prevalence & $\begin{array}{l}\text { Autism population that had the condition at a specific single } \\
\text { point in time }\end{array}$ \\
The Period Autism Prevalence & $\begin{array}{l}\text { The proportion of the population that had Autism any time } \\
\text { within a specific time period }\end{array}$
\end{tabular}

Complex Phenomenon Dimension

A variable with multiple qualitative and quantitative characteristics

Autism Dynamics

A multi-relational (connections) and multi-dimensional (multiple quantitative and qualitative characteristics) interactive process leading to variable representation of Autism manifestations

Complex Phenomenon Paradox

While using rigorous research methods and arrive at contradictory results

Simpleness

Something that has only one dimension.

Categorization of Effectiveness

Different degree of Autism Treatment impact

Primary Autism Complexity

Division

A method for break down the large complexity block into a manageable and focused research

Primary Autism Symptoms

Disruptions in communication, social interaction, and repetitive behavior

Individual Autism Symptoms: Different types and level of intensity of primary Autism symptoms

Autism Etiology Research

Searches for a cause or causes of Autism

Autism Treatment Research

Searches for optimal treatments for variety of symptoms manifestation 
Autism Epidemiology Research

Autism Family Research

Autism Prevention Research

Structural Framework
Searches for reliable system of count and true pattern of Autism prevalence

Uncover the variety of impacts of the Autism on family units

Searches how to prevent Autism

Identification and description of intervention parts and relationship among parts. 
Table A3/1

\section{Appendix 3}

Complete Survey Items for Parents and/or Caregivers

\begin{tabular}{|c|c|}
\hline $\begin{array}{c}\text { Item } \\
\text { Number }\end{array}$ & Item \\
\hline Q1 & What is your relationship to the child? \\
\hline Q2 & How many children do you at The Vista School? \\
\hline Q3 & What is your highest educational degree? \\
\hline Q4 & What was your MAJOR for your highest degree? \\
\hline Q5 & What is the gender of your child who attends The Vista School? \\
\hline Q6 & What is your child's ethnicity? \\
\hline Q7 & At what age (in years) was your child first diagnosed with Autism? \\
\hline Q8 & $\begin{array}{l}\text { Has your child been diagnosed with any of these other disorders? (In addition to, or } \\
\text { previous to ASD) }\end{array}$ \\
\hline Q9 & Does your child take any medications (for symptoms of ASD)? If so, please list them: \\
\hline Q10 & How many years (including this one) has your child been attending The Vista School? \\
\hline Q11 & $\begin{array}{l}\text { Does [or has] your child receive any other treatments for Autism? (Please check the } \\
\text { treatments below and tell us when your child receive them.( In the box - type year)) }\end{array}$ \\
\hline Q13 & $\begin{array}{l}\text { Before I enrolled my child at The Vista School my child attended another program(s) } \\
\text { assisting children with Autism }\end{array}$ \\
\hline Q14 & I feel that my child benefited from the previous program? \\
\hline Q15 & I feel confident that I can describe the goals of the Competent Learner Model (CLM). \\
\hline Q16 & $\begin{array}{l}\text { I am aware that the CLM implementation at The Vista School is developing specific } \\
\text { behavioral repertoires. }\end{array}$ \\
\hline Q17 & $\begin{array}{l}\text { I believe that my child's overall writing skills (such as copying or imitating drawn lines) } \\
\text { have improved as a result of the implementation of the CLM at the Vista School. }\end{array}$ \\
\hline Q18 & $\begin{array}{l}\text { I believe that my child's overall problem solving skills (such as asking for assistance and } \\
\text { manipulating objects) have improved as a result of the implementation of the CLM at the } \\
\text { Vista School. }\end{array}$ \\
\hline Q19 & $\begin{array}{l}\text { I believe that my child's overall reading skills (such as repeating sounds or words read to } \\
\text { him/her) have improved as a result of the implementation of the CLM at the Vista } \\
\text { School. }\end{array}$ \\
\hline Q20 & $\begin{array}{l}\text { I believe that my child's overall observing skills (such as imitating actions) have } \\
\text { improved as a result of the implementation of the CLM at the Vista School. }\end{array}$ \\
\hline Q21 & $\begin{array}{l}\text { I believe that my child's overall talking skills (such as repeating sounds or words and } \\
\text { answering questions) have improved as a result of the implementation of the CLM at the } \\
\text { Vista School. }\end{array}$ \\
\hline Q22 & $\begin{array}{l}\text { I believe that my overall child's listening skills (such as following single-step or series } \\
\text { of directions) have improved as a result of the implementation of the CLM at the Vista } \\
\text { School. }\end{array}$ \\
\hline Q24 & $\begin{array}{l}\text { I believe that overall my child participates more in learning activities (such as following } \\
\text { directions, completing tasks, using a variety of objects, accepting or giving objects to } \\
\text { peers) as a result of the implementation of the CLM at the Vista School. }\end{array}$ \\
\hline
\end{tabular}


Q25 I feel that The Vista School employs rigorous and systematic teaching.

Q26 Overall, how satisfied are you with The Vista School?

Q27 I recommend The Vista School for other children with Autism.

Q28 What do you think is the strongest component of The Vista School?

Q29 Please help us understand in what ways The Vista School can be improved. What would you like to change?

Table A3/2

Complete Survey Items for Administration and Staff and/or Caregivers

\begin{tabular}{|c|c|}
\hline $\begin{array}{c}\text { Item } \\
\text { Number }\end{array}$ & Item \\
\hline Q1 & What is your position at The Vista School? \\
\hline Q2 & What is your Gender? \\
\hline Q3 & What is your ethnicity? \\
\hline Q4 & How many years (including this one) have you been working at The Vista School? \\
\hline Q5 & Overall, how satisfied are you with the CLM Collaborative Consultation? \\
\hline Q6 & Overall, how satisfied are you with the CLM Course of Study? \\
\hline Q7 & Overall, how satisfied are you with the CLM Coaching? \\
\hline Q8 & Overall, how satisfied are you with the Competent Learner Repertoire Assessments? \\
\hline Q9 & Overall, how satisfied are you with the CLM Curriculum? \\
\hline Q10 & Overall, how satisfied are you with the entire CLM? \\
\hline Q11 & The CLM Course of Study materials are easy to use. \\
\hline Q13 & The Competent Learner Model Repertoire Assessments (CLRA) are easy to use. \\
\hline Q14 & The CLM Curriculum materials are easy to use. \\
\hline Q15 & Overall the CLM materials are easy to use. \\
\hline Q16 & $\begin{array}{l}\text { I feel I was well prepared by my coach to use the components of the CLM before I began } \\
\text { using them with my students. }\end{array}$ \\
\hline Q17 & $\begin{array}{l}\text { I feel well supported by my coach when I teach students using the components of the } \\
\text { CLM. }\end{array}$ \\
\hline Q18 & $\begin{array}{l}\text { I feel that I can obtain assistance from my CLM coach with difficult student behavior in } \\
\text { a timely manner. }\end{array}$ \\
\hline Q19 & $\begin{array}{l}\text { I feel that I can obtain assistance from a BCBA with difficult student behavior in timely } \\
\text { manner. }\end{array}$ \\
\hline Q20 & $\begin{array}{l}\text { I am aware that the CLM for children with Autism is developing specific behavioral } \\
\text { repertoires. }\end{array}$ \\
\hline Q21 & $\begin{array}{l}\text { I believe that ALL of my students' overall writing skills (such as copying or imitating } \\
\text { drawn lines) have improved as a result of the implementation of the CLM at the Vista } \\
\text { School. }\end{array}$ \\
\hline Q22 & $\begin{array}{l}\text { I believe that SOME of my students' overall writing skills (such as copying or imitating } \\
\text { drawn lines) have improved as a result of the implementation of the CLM at the Vista } \\
\text { School. }\end{array}$ \\
\hline Q24 & $\begin{array}{l}\text { I believe that ALL of my students' overall problem solving skills (such as asking for } \\
\text { assistance and manipulating objects) have improved as a result of the implementation of } \\
\text { the CLM at the Vista School. }\end{array}$ \\
\hline
\end{tabular}


Q25 I believe that SOME of my students' overall problem solving skills (such as asking for assistance and manipulating objects) have improved as a result of the implementation of the CLM at the Vista School.

Q26 I believe that ALL of my students' overall reading skills (such as repeating sounds or words read to them ) have improved as a result of the implementation of the CLM at the Vista School.

Q27 I believe that SOME of my students' overall reading skills (such as repeating sounds or words read to them) have improved as a result of the implementation of the CLM at the Vista School.

Q28 I believe that ALL of my students' overall observing skills (such as imitating actions, attending to relevant features of objects/pictures or events, and labeling objects or actions) have improved as a result of the implementation of the CLM at the Vista School.

Q29 I believe that SOME of my students' overall observing skills (such as imitating actions, attending to relevant features of objects/pictures or events, and labeling objects or actions) have improved as a result of the implementation of the CLM at the Vista School.

Q30 I believe that ALL of my students' overall talking skills (such as repeating sounds or words and answering questions) have improved as a result of the implementation of the CLM at the Vista School.

Q31 I believe that SOME of my students' overall talking skills (such as repeating sounds or words and answering questions) have improved as a result of the implementation of the CLM at the Vista School.

Q32 I believe that ALL of my overall students' listening skills (such as following single-step or series of directions) have improved as a result of the implementation of the CLM at the Vista School.

Q33 I believe that SOME of my overall students' listening skills (such as following singlestep or series of directions) have improved as a result of the implementation of the CLM at the Vista School.

Q34 I believe that SOME of my overall students' listening skills (such as following singlestep or series of directions) have improved as a result of the implementation of the CLM at the Vista School.

Q35 I believe that overall ALL of my students participate more in learning activities (such as following directions, completing assignments, using a variety of objects, or accepting or giving objects to peers) as a result of the implementation of the CLM at the Vista School.

Q36 I believe that overall SOME of my students participate more in learning activities (such as following directions, completing assignments, using a variety of objects, or accepting or giving objects to peers) as a result of the implementation of the CLM at the Vista School.

Q37 I feel that The Vista School employs rigorous and systematic teaching.

Q38 I feel comfortable using the CLM Curriculum Instructional Formats.

Q39 I am using CLM support materials in my classroom:

Q40 What do you think is the strongest component of the CLM?

Q41 Please help us understand in what ways the CLM can be improved. Tells us what you would like to change? 
Table A3/3

Transcription of Parents Answers: Strongest Component of The Vista School

\section{What do you think is the strongest component of The Vista School?}

They treat my child as a whole. No one had ever successfully addressed his inappropriate behaviors before he started at The Vista School. In the Intermediate Unit, they were only supposed to focus on the academics. It became clear that without help with the negative behaviors, my son would never be successful academically. The autism classroom was understaffed and they receive very limited, inadequate training in relation to the staff at Vista. They would try to take data, create an FBA, and then come up with a behavior plan, but they usually didn't work. Then, mostly because the school psychologist is far too overworked, she wouldn't return to revise the FBA and my son would get lost in the shuffle. Also, rarely did they have the resources to come up with a good plan. They simply don't have enough training in the area of managing behaviors. Most of the time they would send him to the calming corner for as long as he needed--sometimes most of the day. The Vista School addresses my son's behaviors, responds appropriately when he's upset, teaches him coping techniques, and also teaches us at home how to respond similarly so there is consistency. Because of this, he is now also making tremendous progress in other areas. He's speaking in sentences most of the time, whereas he only occasionally spoke in a sentence before Vista. He asks for help. He calls for me when I'm out of the room. He doesn't scream in the morning because he has to go to the school. This has only been since September! They know what they are doing, they go beyond what anyone has ever done for my child, and they are willing to share their knowledge and train anyone who has contact with my son. We are so very grateful for their expertise, and their patience, and the commitment they make to helping my son and our family have a better life. I would recommend The Vista School to anyone who cares for a child with autism. I truly can't thank them enough.

$>$ The amazing staff

$>$ Staff and how they work with parents-raising the bar always for my son 
The staff makes the school successful . Each staff member is well trained and cares greatly about their students. It's the atmosphere when you walk into the school that you notice first. It's pleasant and welcoming. My son has improved so much behaviorally and academically since attending the Vista School. He actually wants to go to school. He has a strong relationship with his classroom staff. You can see how the staff respects their students and how they respond in return. Having my son attend Vista is a blessing and I don't take it for granted!

$>$ I believe the strongest component is the use of ABA.

$>$ How they work and teach my child with the pec program and how they work individual with my child. he needs lots of one on one and hand over hand, they are able to teach him things. also the training they have on working with my child. I was told he will never talk or understand the use of toileting. this boy in a few months learned how to void in the toilet. he is now 13 it is a joy and a great help that the school was able to master this for my son. I love the school in so many ways!!!!!!

$>$ Staff dedication

$>$ The dedication of the staff. NO program no matter how well thought out can do anything if the delivery method is lacking. The delivery method in this case the is the staff, their love of our children, their dedication to our children and our families. Without the staff Vista would just be another school for autistic support instead of the THIRD RANKED SCHOOL IN THE COUNTRY!!!

$>$ Work systematically to achieve each individual goal.

BA curriculum taught by highly qualified and highly dedicated staff.

The staff and teachers, aides, everyone works together and really helps our children learn and they are all very involved in making sure they grow. The staff are awesome, from the bus drivers getting him to school, the community interactions, the life skills and other training is light years ahead of where he was. Kendra Peacock was instrumental in supporting our ability to get him there and the staff and everyone at every IEP meeting always keeps us informed on all areas. We are $100 \%$ pleased with Vista and the CLM model/

Teaching and behavior technician staff

$>$ The staff who truly care for and love our students. Yes, the teaching is great, but without the dedicated and passionate staff, Vista wouldn't have the draw and success that it does. 
The personnel. Their commitment to the model and to improve their skills. Their love of the children and dogged determination to get the best out of them

$>$ Caring staff, very structured learning that takes into consideration every child's needs.

$>$ The sole focus on autism (as opposed to trying to incorporate multi-disabilities). The staff commitment to helping kids improve

$>$ I believe Vista's strongest component is the camaraderie amongst all staff, administration and families. There are no outside agencies with different agendas than the classroom. Everyone is on the same agenda and works together to help make our kids successful!

$>$ Compassion

$>$ Professionalism, Communication and interaction with parents.

$>$ Behavioral support and training

$>$ The loving, caring environment and wonderful staff!

$>$ Dedicated and caring staff in a safe environment

$>$ Most of the staff seem to have a genuine interest in the success of the students

$>$ Comprehensive, systematic approach to behavioral management.

$>$ One on one behavioral therapists

$>$ Its an all in one program. The latest teaching/learning methods. Its a one stop get it all for your child. Everything you need under 1 roof. The BEST program in all of Central Pennsylvania for children with moderate to severe Autism Spectrum Disorder.

$>$ Responsiveness to parents' needs

> The Staff who work directly with my child. The credentials and personalities of the staff are by far the best I've seen. They truly care about the child. The staff also makes it a priority to include the family as part of the child's team 
Table A3/4

Transcription of Parents Answers: In What Ways can be The Vista School improved

\section{Please help us understand in what ways The Vista School can be improved. What would you like to change?}

My son has only been at The Vista School for a few months. The only thing I can think of to change is regarding communication with the other parents. Vista does an exceptional job of offering trainings and organizing meetings where parents can meet and talk, but I'd like to be able to connect with other parents in a more casual way, if possible, without having to meet in person. I was hoping there was a place online, but I no one seems to know. I feel this would be helpful.

$>$ Very thankful for additional focus on transition age issues and opportunities after Vista. Increased focus here would always be great.

$>$ Have a work=living program for students when they graduate

$>$ Longer school days

$>$ I'd like to see more seminars, workshops.., for parents. Is like communication be a bit stronger between teachers and parents.

$>$ I do feel like my son's rate of acquisition is slower in the CLM program than in Verbal Behavior. However, I also see how CLM addresses some skills that his VB program has not. I am hoping the combination of the two will result in the best of both worlds for my son.

At this point there is not much to change. if anything I would say dr note hand in. we have to mail them, but I don't understand why I cant send in with my son to school when he returns the following day. sending dr notes is a struggle sometimes due to forgetting or getting loss before I can send.

$>$ Add a day program

Honestly, I can't think of a thing. Vista support staff, board of directors all know EXACTLY what they are doing. I can't offer anything.

$>$ Bring in a music therapist once a week to the classrooms

$>$ Expanded after school services or integration of school programming to after school programming implemented by outside agencies 
Nothing we can think of, it is very well staffed, planned, and everyone is very responsive on any concerns, we have always felt like equal partners in the process and are so pleased our son will graduate this year

Reliance on a large number of adults to deliver services. Would like to see more tapering of services as the child ages to promote skills needed for success in future, less intensive services.

Bigger enrollment. pre-school programming. Adult services.

$>$ I cannot think of any improvements

$>$ Would like more use of technology in teaching - teaching from video for example since many of our kids our very visual. Lessons can be reused as well across students. In addition, could be used in the home setting as well.

$>$ The ONLY thing I can suggest is to get the adult program up and running. It is so important for our children to have a strong program as we have now, but after 21 where resources are so limited! I cannot imagine what will happen to my son without the support and strength of Vista. It is an amazing program!

Maybe sending some homework assignments. Not a lot.

$>$ More literacy based programming.

$>$ Create an adult program that current Vista students could attend.

$>$ Consult Richard Foxx on a regular basis.

D Nothing!!!! Vista is the best in everything !!!!! Two week breaks are rough on families!

$>$ Our family is very satisfied with the Vista School and have no suggestions.

$>$ Better communication with the personal at school I would like those him at school to see how he responds there

More involvement with sports and Special Olympics

$>$ I'm wishful and hopeful the over 21 program will be in place soon, as well as Residential care. That's the only change I would like to see. Vista providing the same quality care for adults and possible residents.

$>$ More feedback from therapists

Perhaps one suggestion would be to use layman's terms when describing programs, treatments and results with parents. Sometimes paperwork will come home with requests for signatures 
of receipt, etc. and we don't understand the terminology to be able to decide whether we agree or not. 
Table A3/5

Transcription of Staff Answers: Strongest Component of the CLM

\section{What do you think is the strongest component of the CLM? (Exact Text Responses)}

Use of visual poster boards (scope and sequence, first 7 lessons) - staff tend to go to them quickly and use them as reference tools

$>$ All staff can replicate exactly the same targets, and even probe a step harder, keeping the same target in mind. I also really like that they can work on "just echoics" or "Just leisure" but a single activity can be used to gleen both targets. Often we say "Oh he's playing well, but we overlook the fact that he doesn't make sounds or echo/imitate when playing. All the skills work together to create meaningful life

$>$ Repertoires

$>$ Identifying and outlining the 7 repertoires of the competent learner

$>$ Repetition in teaching the materials, which strengthens many components of the CLM

$>$ Lessons Breakdown

$>$ Collaborative consultations

$>$ All of the tools that help you develop the best learning environment and instruction for the students and the staff.

$>$ How all the repertoires work together

$>$ Consistency in teaching and behavioral theory across teams and instructors

$>$ The formats that help create consistency in teaching across staff

$>$ CLM is able to identify where students would be according to age level

$>$ Training for adult learners, CLRA

$>$ Teaching participation

$>$ Problem Solver

$>$ The ability if the curriculum to be adapted for students to such varying functional levels

$>$ Formats to follow are fairly easy to follow with some initial instruction

$>$ Slow and systematic positive support of learners. Making a fun, accommodating, and preferred learning environment. 
The formats

$>$ Participation areas - 501/503

$>$ The early lessons for more naive learners

$>$ The curriculum

$>$ Participator repertoire

$>$ Being able to look at a learners strengths and use them to teach to their needs

$>$ Lessons are laid out to tell you as an instructor what to do and what to expect from your learner

$>$ Problem solver

$>$ The constructivist approach to teaching skills

$>$ Understanding the repertoires and how they are all intertwined

$>$ Teaching of communication skills (talker repertoire). Also, the coaching system and accountability to other instructors

$>$ The coaching

$>$ Repertoires

$>$ Coaching support and assistance

$>$ The scope and sequence and how all of the repertoires work together as the student moves up through the lessons. 
Table A3/6

Transcription of Staff Answers: In What Ways CLM can be Improved

\section{Please help us understand in what ways the CLM can be improved. Tells us what you would like to change?}

Clean up the server so the tools are easily accessible and able to be found ensure all staff are assigned a coach and have direct time with a coach - may require less coach to staff ratio to make sure everyone is getting individualized time

$>$ Clarification on the variety of materials during the .505 track (leisure). When targeting one item for a duration, or even when a bin is used and the student only PICKS one item, regardless of their value in the item, how can you better rearrange. How often to use similar tasks to test multiple tracks. I feel like we teach a kid to imitate by using objects, but it doesn't take but 2 days for those objects now to be associated with the action that we error corrected using a prompt fading strategy. ... Maybe more clarification on how to error correct by continued to require the response in THAT same repertoire. (i.e.: a child is to imitate a 2 arms up overhead, but they only imitate $1 \mathrm{arm})$. Instead of giving a verbal direction with the error correction or prompt fading, how can be target the specific deficit that caused their error (i.e.: didn't attend to the direction to "do what I do"[listener] or if they error because their physical disability requires an approximated response (then target the problem solver to teach how to manipulate their own body).

$>$ Using CLM with transition-age students

$>$ Have the Staff do the different units in a timely manner, to more understand the lessons of the students

$>$ N/A

Amount of time spent with coaches. Checkouts happen few and far between

More user friendly manual that lays out everything- so that the CLM Curriculum is more sustainable across time

More specific data collection methods would be beneficial

Increased education on ways to alter the curriculum when a student is erroring or not progressing in a common way. 
I like everything about CLM

$>$ I do not think that CLM is geared towards older students who are able to work for half hour to hour blocks of time. Most of CLM is focused on reinforcing students after a few minutes of a task. The focus for high school students does not always need to be on "rates of reinforcement", instead high school students benefit from activities that are motivating in themselves. ABI should be more of a focus than reinforcement rates.

$>$ Coaching across all instructional staff is impossible with the number of coaches Vista currently has. Additional coaches must be developed in order to continue to implement and grow with CLM.

$>$ Less programing for each student. It is difficult for BTs to remember all of the different programs for each learner and It seems that teachers have difficulty keeping up with the updates. Use the programs as building blocks instead of trying to run as many as possible.

More support from classroom coaches and bcba's. Both currently absent from room almost completely.

$>$ Online videos rarely work. Scheduling of checkouts and applications must be improved

> A hierarchy of educational and behavioral interventions that starts with CLM at the top and a guideline of when and how to include other evidence-based practices. Also, a curriculum of what to teach that compliments CLM's how to teach it.

$>$ Better explaining and reasoning on how to use documents such as learner profiles, reinforce assessments, etc.

$>$ I would like our classroom to have a classroom coordinator to check out my BTs through the CLM units and be available to coach my BTs through the units and to help to better understand the CLM components on the lesson plans

$>$ The higher lessons and it's use with higher functioning students

$>$ I would like to improve the acquisition of the course of study content in paraprofessionals. Specifically, the guided practices and application exercises in which the adult learner should be brought to mastery of the content, is currently inadequate

$>$ The CLRA could be written in a more objective way 
I think that course of study online is very lengthy and overwhelming. I think there definitely needs to be exposure to the materials before checking out with my coach, but I do not learn best online; so, I get overwhelmed with the modules and the questions.

$>$ After finishing a unit, application and meeting with coach would be done within a few days. Once you have finished a unit only doing the 1 on 1 process once then reviewing later as needed when questions arise.

$>$ A greater focus on integrating academics into repertoires. Easier/better written standards.

$>$ The entire method of teaching imitation and sorting. The computer program for training is frustrating to use. The way it allows academics to fall by the wayside if the teacher isn't also keeping up with that.

$>$ The video components of the online course of study are horrible. They are out of date and tend to have incorrect information in them in my opinion. I learn very little from them.

$>$ Ease of use with forms, less cumbersome

$>$ Having trainers in the classroom on a daily basis. We simply do not get enough "in the moment" training in the classroom. IN ORDER TO IMPLEMENT THE PROGRAM WITH FIDELITY $<$ WE HAVE TO BE TRAINED WITH FIDELITY. 


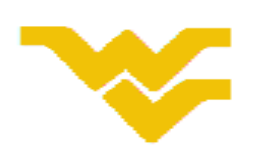

\section{West Virginia University}

\section{College of Education and Human Services}

\section{Dear Participant,}

This letter is a request for you to take part in a dissertation research project to evaluate the effectiveness of the Competent Learner Model (CLM) at The Vista School. Dana Cihelkova is conducting this project as a doctoral student at West Virginia University under the supervision of Dr. Dan Hursh, a professor of Educational Psychology at West Virginia University. Your participation in this project is greatly appreciated and will take approximately 15 minutes for you to fill out the electronic and anonymous survey.

Your involvement in this project will be kept as confidential as legally possible. All data will be reported in the aggregate. You must be 18 years of age or older to participate. You will not be asked for any information that may lead back to your identity as a participant. Your participation is completely voluntary - you can decide to fill out or not to fill out the survey. You may skip any question that you do not wish to answer and you may discontinue at any time. Your child's class standing will not be affected if you decide either not to participate or to withdraw. West Virginia University's Institutional Review Board acknowledgement of this project is on file.

I hope that you will participate in this research project, as it could be beneficial in understanding the impact of the Competent Learner Model on your child's life. Thank you very much for your time. Should you have any questions about this letter or the research project, please feel free to contact Dana Cihelkova at (304) 293-2146 or by email at Dana.Cihelkova@mail.wvu.edu. Dan Hursh, Ph.D., (304) 293-2076 or by e-mail at Dan.hursh@mail.wvu.edu

Thank you for your time and help with this project.

Sincerely,

Dana Cihelkova and Dan Hursh

Department of Learning Sciences and Human Development Instructional Design \& Technology, Educational Psychology, and Child Development \& Family Studies 


\section{West Virginia University}

\section{College of Education and Human Services}

Dear Participant,

This letter is a request for you to take part in a dissertation research project to evaluate the effectiveness of the Competent Learner Model (CLM) at The Vista School. Dana Cihelkova is conducting this project as a doctoral student at West Virginia University under the supervision of Dr. Dan Hursh, a professor of Educational Psychology at West Virginia University. Your participation in this project is greatly appreciated and it will take you approximately 15 minutes to fill out the electronic and anonymous survey.

Your involvement in this project will be kept as confidential as legally possible. All data will be reported in the aggregate. You must be 18 years of age or older to participate. You will not be asked any information that may lead back to your identity as a participant. Your participation is completely voluntary - you can decide to fill out or not to fill out the survey. You may skip any question that you do not wish to answer and you may discontinue at any time. Your employment standing will not be affected if you decide either not to participate or to withdraw. West Virginia University's Institutional Review Board acknowledgement of this project is on file.

I hope that you will participate in this research project, as it could be beneficial in understanding the impact of the Competent Learner Model on your students' life. Thank you very much for your time. Should you have any questions about this letter or the research project, please feel free to contact Dana Cihelkova at (304) 293-2146 or by email at Dana.Cihelkova@mail.wvu.edu or Dan Hursh, Ph.D., (304) 293-2076 or by email at Dan.Hursh@mail.wvu.edu.

Thank you for your time and help with this project.

Sincerely,

Dana Cihelkova and Dan Hursh

Department of Learning Sciences and Human Development

Instructional Design \& Technology, Educational Psychology, and

Child Development \& Family Studies 
Evaluation Booklet for the CLM Developer: Plan of the Evaluation and Pilot Surveys

Evaluation Booklet for the Developer of the Competent Learner Model Plan for the Comprehensive Evaluation

At The Vista School

And Pilot Surveys Items 


\section{Dynamic Evaluation Model for CLM}

\begin{tabular}{|c|c|c|c|}
\hline Phase & Objective & Instrument & Data Analysis \\
\hline Conceptualization & What is the CLM constellation & $\begin{array}{l}\text { Theoretical Construct } \\
\text { Development of visual representation } \\
\text { of the intervention (handout) }\end{array}$ & $\begin{array}{l}\text { Development of Theory of Change } \\
\text { How many parts are there in the CLM? } \\
\text { What is the intensity of CLM (time)? } \\
\text { What are the functional relations among } \\
\text { the parts of the CLM? } \\
\text { What are the CLM strategies? } \\
\text { What are the CLM assumptions? } \\
\text { What are the CLM short-term outcomes? } \\
\text { What are the CLM intermediate } \\
\text { outcomes? } \\
\text { What are the CLM ultimate outcomes? }\end{array}$ \\
\hline Diagnostics & $\begin{array}{l}\text { To collect specialized demographic } \\
\text { data and symptoms } \\
\text { Gender } \\
\text { Age } \\
\text { Race } \\
\text { Parents Occupation } \\
\text { First Diagnosed } \\
\text { Symptoms }\end{array}$ & $\begin{array}{ll} & \text { Quantitative Standardized } \\
& \text { Measurement [GARS -3] } \\
& \\
\circ & \text { Restrictive/Repetitive } \\
& \text { Behaviors } \\
\circ & \text { Social Interaction } \\
\circ & \text { Social Communication } \\
\circ & \text { Emotional Responses } \\
\circ & \text { Cognitive Style } \\
\circ & \text { Maladaptive Speech. }\end{array}$ & $\begin{array}{ll}\text { Descriptive Statistics } \\
\text { - } & \text { Means } \\
\text { - } & \text { Standard Deviations } \\
\text { - } & \text { Frequencies Tables }\end{array}$ \\
\hline Environment & To assess the contextual factors & Quantitative Assessment & $\begin{array}{l}\text { Descriptive Statistics } \\
\text { - Means }\end{array}$ \\
\hline
\end{tabular}




\begin{tabular}{|c|c|c|c|}
\hline & & $\begin{array}{l}\quad \text { [Cross-Sectional Survey for } \\
\quad \text { stakeholders] } \\
\text { Place } \\
\text { Time } \\
\text { Form } \\
\text { Cost } \\
\text { Intensity of the treatment } \\
\text { Learners' background } \\
\text { - Family } \\
\text { - School }\end{array}$ & $\begin{array}{ll}\text { - } & \text { Standard Deviations } \\
\text { - } & \text { Frequencies Tables } \\
\text { - } & \text { Correlations }\end{array}$ \\
\hline Outcomes & $\begin{array}{l}\text { Determine the actual capacity } \\
\text { (impact) of the CLM } \\
\text { What is the degree, form, and speed } \\
\text { of the changes in behavior }\end{array}$ & \begin{tabular}{ll} 
& \multicolumn{1}{c}{ Quantitative Standardized } \\
& Measurement \\
& [Vineland -II and (PLSTM-5)] \\
& \\
$\circ$ & Communication \\
$\circ$ & Daily Living Skills \\
$\circ$ & Socialization \\
$\circ$ & Motor Skills \\
$\circ$ & Maladaptive Behavior Index \\
$\circ$ & Auditory Comprehension \\
$\circ$ & Expressive Communication
\end{tabular} & $\begin{array}{l}\text { Descriptive Statistics } \\
\text { - } \quad \text { Means } \\
\text { - Standard Deviations } \\
\text { Inferential Statistics } \\
\text { - Dependent } t \text { - test } \\
\text { Kolmogorov-Smirnov Test } \\
\text { Levene's Test }\end{array}$ \\
\hline Understanding & \multicolumn{3}{|c|}{$\begin{array}{l}\text { Expand our knowledge about the Competent Learned Model } \\
\text { Provide a contextual understanding of effectiveness of the Competent Learned Model } \\
\text { Identify who benefits the most from the Competent Learned Model } \\
\text { Identify which children can benefit the most from what structures of the Competent Learned Model } \\
\text { Autism Symptoms Distribution [tabulation of symptoms, their intensity and frequency] at the Vista School } \\
\text { Dynamics of Autism symptomology [the relationships among symptoms] at the Vista School }\end{array}$} \\
\hline
\end{tabular}




\section{Conceptualization Phase What is the CLM constellation?}




\section{Visual Representation of the CLM Intervention}

Please fill out the following ...

\begin{tabular}{|l|l|l|}
\hline Parts $^{23}$ & Relations $^{24}$ & Strategies $^{25}$ \\
\hline & & \\
\hline & & \\
\hline & & \\
\hline & & \\
\hline & & \\
\hline & & \\
\hline
\end{tabular}

${ }^{23}$ How many parts are there in the CLM?

${ }^{24}$ What are the functional relations among the parts of the CLM?

${ }^{25}$ What are the CLM strategies? 


\begin{tabular}{|l|c|c|}
\hline \multicolumn{2}{|c|}{ Outcomes } \\
\multicolumn{2}{|c|}{ Please fill out the following ... } \\
\hline Short-Term & \multicolumn{1}{|c|}{ Intermediate } & \multicolumn{2}{c|}{ Ultimate } \\
\hline & & \\
\hline & & \\
\hline & & \\
\hline & & \\
\hline & & \\
\hline
\end{tabular}

${ }^{26}$ What are the CLM short-term outcomes

${ }^{27}$ What are the CLM intermediate outcomes?

${ }^{28}$ What are the CLM ultimate outcomes? 


\begin{tabular}{|l|l|}
\hline \multicolumn{1}{|c|}{ Intensity $^{29}$} & \multicolumn{1}{c|}{$\begin{array}{c}\text { Assumptions }^{30} \\
\text { Please fill out the following ... }\end{array}$} \\
\hline & \\
\hline & \\
\hline & \\
\hline & \\
\hline & \\
\hline & \\
\hline
\end{tabular}

29 What is the intensity of CLM? (Hours per week)

${ }^{30}$ What are the CLM assumptions? 


\section{Diagnostics Phase}

Specialized Demographic and Symptoms Representation 


\section{Diagnostics Phase}

\section{Surveys items for parents and/or caregivers}

Dear Parents, Legal Guardians and Caregivers,

This anonymous survey is your opportunity to expresses your opinions, feelings and satisfaction with the Competent Learner Model (CLM) that is fully implemented in The Vista School. Please, help us to understand in what ways the CLM is working and in what ways it can be improved by filling out this brief survey. Some of the survey items (demographics, education, medical and/or other psychological issue) are designed to contribute to the national survey for advance Autism Research knowledge and understating.

Thank YOU!

Dana Cihelkova and Dan Hursh

West Virginia University 


\section{What is your role?}

$\square$ Mother

$\square$ Father

$\square$ Legal Guardian

$\square$ Caregiver

$\square$ Other

2. What is your highest educational degree?

$\square$ High School Diploma

$\square \mathrm{BA}$

$\square$ MA

$\square \mathrm{MS}$

$\square \mathrm{MD}$

$\square \mathrm{PhD}$

$\square$ Other

3. What was your major? 
4. What is the gender of your child who attends The Vista School?

$\square$ Female

$\square$ Male

\section{What is your child's ethnicity?}

$\square$ Native American (American Indian)

$\square$ Pacific Islander (Samoan, Filipino, etc.)

$\square$ White, non-Latino

$\square$ White, Latino (or Hispanic)

$\square$ Black, African American

$\square$ Asian: Chinese, Japanese

$\square$ South Asian: Vietnamese, Cambodian, Hmong, Laotian, Chinese Vietnamese

$\square$ Other Asian: (Not Chinese, Japanese, or South Asian)

6. What age ( in years) your child was first diagnosed with Autism?

$\square 1$

$\square 2$

$\square 3$

$\square 4$ 
$\square 6$

$\square 7$

$\square 8$

$\square 9$

$\square 10$

$\square 11$

$\square 12$

$\square$ Other

7. Has your child been diagnosed any of these other disorders?

$\square$ Language Disorder

$\square$ Cognitive Delay

$\square$ Anxiety Disorder

$\square$ Mood Disorder

$\square$ Depression Disorder

$\square$ Attention Disorder (ADHD)

$\square$ Neurological Disorder (Subscale: what type)

$\square$ Learning Disorder (Subscale: what type)

$\square$ Medical Issue (Subscale: what type: GI, Sleep, Genetic, Bowel Disease)

$\square$ Tic Disorder

$\square$ Fragile X Syndrome 
$\square$ Epilepsy

$\square$ Mendelian

$\square$ Bipolar Disorder

$\square$ Other

8. Does your child take any medications? If so, please list them below.

\section{How many years (including this one) has your child been attending The Vista School?}

$\square 1$

$\square 2$

$\square 3$

$\square 4$

$\square 5$

$\square 6$

$\square 7$

$\square 8$

$\square 9$

$\square 10$

$\square 11$ 
10. Does or has your child receive any other treatments regarding Autism? If so, please list them below and tell us when they receive(d) them.

$\square$ Behavioral Based Treatment (ABA) (Subscale: what type:....

$\square$ Psychological Based Treatments (Relational) (Subscale: what type:....

$\square$ Educational Based Treatments (Behavioral) (Subscale: what type:....

$\square$ Neural Based Treatments (Brain) (Subscale: what type:....

$\square$ Medical Based Treatments (Pharmacological) (Subscale: what type:....

$\square$ Biological Based Treatments (Nutritional) (Subscale: what type:....

$\square$ Other

Environment Phase Items but they will be placed in one survey so that parents will have only one survey to fill out ......

11. How many years (including this one) has your child been attending The Vista School?

$\square 1$

$\square 2$

$\square 3$

$\square 4$

$\square 5$

$\square 6$ 
$\square 8$

$\square 9$

$\square 10$

$\square 11$

12. Before I enrolled my child at The Vista School my child attended another program(s) assisting children with Autism

$\square$ Yes (Drop down menu... what kind)

$\square$ No

$\square$ I don't know

13. I know a lot about the Competent Learner Model (CLM).

$\square$ Strongly Agree

$\square$ Agree

$\square$ Somewhat Agree

$\square$ Neutral

$\square$ Somewhat Disagree

$\square$ Disagree

$\square$ Strongly Disagree 
14. I know what my child is taught when the Vista School implements the Competent Learner Model.

$\square$ Strongly Agree

$\square$ Agree

$\square$ Somewhat Agree

$\square$ Neutral

$\square$ Somewhat Disagree

$\square$ Disagree

$\square$ Strongly Disagree

\section{Overall, how satisfied are you with The Vista School?}

$\square$ Strongly Satisfied

$\square$ Satisfied

$\square$ Somewhat Satisfied

$\square$ Neutral

$\square$ Somewhat Satisfied

$\square$ Dissatisfied

$\square$ Strongly Satisfied 
16. I am aware that the CLM implementation at The Vista School is developing specific behavioral repertoires.

$\square$ Strongly Agree

$\square$ Agree

$\square$ Somewhat Agree

$\square$ Neutral

$\square$ Somewhat Disagree

$\square$ Disagree

$\square$ Strongly Disagree

17. I believe that my child's writing (applicable) skills have improved as a result of the implementation of the CLM at the Vista School.

$\square$ Strongly Agree

$\square$ Agree

$\square$ Somewhat Agree

$\square$ Neutral

$\square$ Somewhat Disagree

$\square$ Disagree

$\square$ Strongly Disagree 
18. I believe that my child's problem solving (applicable) skills have improved as a result of the implementation of the CLM at the Vista School.

$\square$ Strongly Agree

$\square$ Agree

$\square$ Somewhat Agree

$\square$ Neutral

$\square$ Somewhat Disagree

$\square$ Disagree

$\square$ Strongly Disagree

19. I believe that my child's reading (applicable) skills have improved as a result of the implementation of the CLM at the Vista School.

$\square$ Strongly Agree

$\square$ Agree

$\square$ Somewhat Agree

$\square$ Neutral

$\square$ Somewhat Disagree

$\square$ Disagree

$\square$ Strongly Disagree 
20. I believe that my child's observing (applicable) skills have improved as a result of the implementation of the CLM at the Vista School.

$\square$ Strongly Agree

$\square$ Agree

$\square$ Somewhat Agree

$\square$ Neutral

$\square$ Somewhat Disagree

$\square$ Disagree

$\square$ Strongly Disagree

21. I believe that my child's talking (applicable) skills have improved as a result of the implementation of the CLM at the Vista School.

$\square$ Strongly Agree

$\square$ Agree

$\square$ Somewhat Agree

$\square$ Neutral

$\square$ Somewhat Disagree

$\square$ Disagree

$\square$ Strongly Disagree 
22. I believe that my child's listening (applicable) skills have improved as a result of the implementation of the CLM at the Vista School.

$\square$ Strongly Agree

$\square$ Agree

$\square$ Somewhat Agree

$\square$ Neutral

$\square$ Somewhat Disagree

$\square$ Disagree

$\square$ Strongly Disagree

23. I believe that my child participates more in learning activities as a result of the implementation of the CLM at the Vista School.

$\square$ Strongly Agree

$\square$ Agree

$\square$ Somewhat Agree

$\square$ Neutral

$\square$ Somewhat Disagree

$\square$ Disagree

$\square$ Strongly Disagree 
24. I feel that The Vista School employs rigorous and systematic teaching.

$\square$ Strongly Agree

$\square$ Agree

$\square$ Somewhat Agree

$\square$ Neutral

$\square$ Somewhat Disagree

$\square$ Disagree

$\square$ Strongly Disagree

\section{I recommend The Vista School for other children with Autism.}

$\square$ Strongly Agree

$\square$ Agree

$\square$ Somewhat Agree

$\square$ Neutral

$\square$ Somewhat Disagree

$\square$ Disagree

$\square$ Strongly Disagree 
26. Pleases help us understand in what ways The Vista School can be improved. What would you like to change?

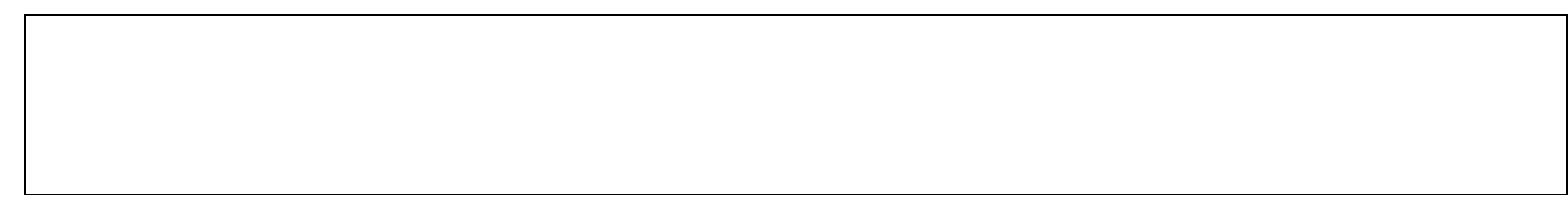

27. What do you think is the strongest component of the Vista School?

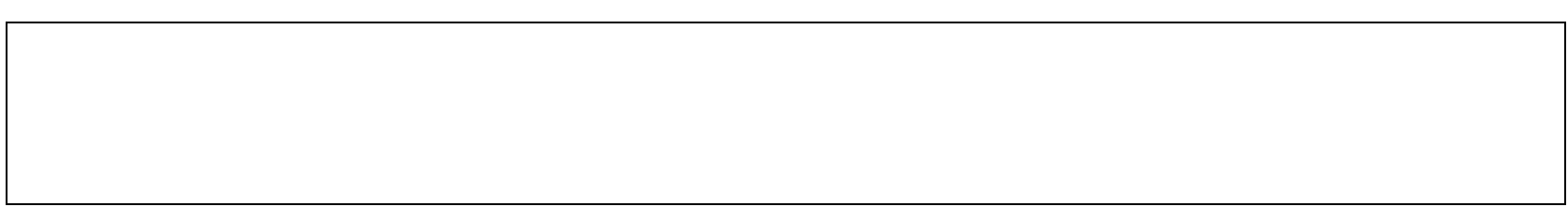




\section{Symptoms GARS-3}

\begin{tabular}{|c|l|c|}
\hline \multicolumn{1}{|c|}{ STEREOTYPED BEHAVIOR } & DATA ANALYSES \\
\hline $\mathbf{1}$ & Avoids eye contact/looks away & M, SD, Frequency \\
\hline $\mathbf{2}$ & Stares at hands, objects & M, SD, Frequency \\
\hline $\mathbf{3}$ & Flicks fingers rapidly & M, SD, Frequency \\
\hline $\mathbf{4}$ & Eats specific foods & M, SD, Frequency \\
\hline $\mathbf{5}$ & Licks, tastes, inedibles & M, SD, Frequency \\
\hline $\mathbf{6}$ & Smells/sniffs objects & M, SD, Frequency \\
\hline $\mathbf{7}$ & Whirls, turns in circles & M, SD, Frequency \\
\hline $\mathbf{8}$ & Spins objects & M, SD, Frequency \\
\hline $\mathbf{9}$ & Rocks back and forth & M, SD, Frequency \\
\hline $\mathbf{1 0}$ & Rapid lunging/darting & M, SD, Frequency \\
\hline $\mathbf{1 1}$ & Prances & M, SD, Frequency \\
\hline $\mathbf{1 2}$ & Flaps hands & M, SD, Frequency \\
\hline
\end{tabular}




\begin{tabular}{|c|l|c|}
\hline Item & STEREOTYPED BEHAVIOR & DATA ANALYSES \\
\hline $\mathbf{1 5}$ & Repeats words & M, SD, Frequency \\
\hline $\mathbf{1 6}$ & Repeats out of context & M, SD, Frequency \\
\hline $\mathbf{1 7}$ & Repeats over and over & M, SD, Frequency \\
\hline $\mathbf{1 8}$ & Speaks/signs with flat tone/affect & M, SD, Frequency \\
\hline $\mathbf{1 9}$ & Responds inappropriately & M, SD, Frequency \\
\hline $\mathbf{2 0}$ & Looks away when called & M, SD, Frequency \\
\hline $\mathbf{2 1}$ & Does not ask for things & M, SD, Frequency \\
\hline $\mathbf{2 2}$ & Does not initiate conversation & M, SD, Frequency \\
\hline $\mathbf{2 3}$ & Uses 'yes' and 'no' inappropriately 04 29 52 15 & M, SD, Frequency \\
\hline $\mathbf{2 4}$ & Uses pronouns inappropriately -07 44 6900 & M, SD, Frequency \\
\hline $\mathbf{2 5}$ & Uses the word I inappropriately 01 44 73-01 & \\
\hline $\mathbf{2 6}$ & Repeats unintelligible sounds 58 04 34 -22 & \\
\hline $\mathbf{2 7}$ & Uses gestures instead of speech & \\
\hline $\mathbf{2 8}$ & Inappropriately answers about story & \\
\hline
\end{tabular}

\begin{tabular}{|c|l|c|}
\hline Item & \multicolumn{1}{|c|}{ SOCIAL INTERACTION } & DATA ANALYSES \\
\hline $\mathbf{2 9}$ & Avoids eye contact & M, SD, Frequency \\
\hline $\mathbf{3 0}$ & Stares/looks unhappy when praised & M, SD, Frequency \\
\hline $\mathbf{3 1}$ & Resists physical contact & M, SD, Frequency \\
\hline $\mathbf{3 2}$ & Does not imitate & M, SD, Frequency \\
\hline $\mathbf{3 3}$ & Withdraws/remains aloof & M, SD, Frequency \\
\hline $\mathbf{3 4}$ & Unreasonably fearful & M, SD, Frequency \\
\hline $\mathbf{3 5}$ & Unaffectionate & M, SD, Frequency \\
\hline $\mathbf{3 6}$ & No recognition (looks through people) & M, SD, Frequency \\
\hline $\mathbf{3 7}$ & Laughs, giggles, cries inappropriately & M, SD, Frequency \\
\hline $\mathbf{3 8}$ & Uses toys/objects inappropriately & M, SD, Frequency \\
\hline $\mathbf{3 9}$ & Does things repetitively/ritualistically & M, SD, Frequency \\
\hline $\mathbf{4 0}$ & Upset when routines changed & \\
\hline
\end{tabular}


41 Tantrums when given commands

M, SD, Frequency

$\mathbf{4 2}$ Lines up objects, upset when disturbed 


\section{Environment Phase}

Assessment of the contextual factors 


\section{Environment Phase}

\section{Survey items for teachers and administration}

Dear Staff Members,

This anonymous survey is your opportunity to expresses your opinions, feelings and satisfaction with the CLM that is fully implemented in The Vista School. Please, help us to understand in what ways the CLM is working or in what ways it can be improved by filling out this brief survey. Some of the survey items (demographics) are designed to contribute to the national survey for advance Autism Research knowledge and understating.

Thank YOU!

Dana Cihelkova and Dan Hursh

West Virginia University 


\section{What is your position at The Vista School?}

$\square$ Teacher

$\square$ Administration

$\square$ Other

\section{What is your Gender?}

$\square$ Female

$\square$ Male

\section{What is your ethnicity?}

$\square$ Native American (American Indian)

$\square$ Pacific Islander (Samoan, Filipino, etc.)

$\square$ White, non-Latino

$\square$ White, Latino (or Hispanic)

$\square$ Black, African American

$\square$ Asian: Chinese, Japanese

$\square$ South Asian: Vietnamese, Cambodian, Hmong, Laotian, Chinese Vietnamese

$\square$ Other Asian: (Not Chinese, Japanese, or South Asian) 
Please, indicate the extent to which you Strongly Agree or Strongly Disagree with following questions regarding the CLM curriculum at The Vista School. Check one option that most closely reflects you or your opinion.

\section{How many years (including this one) your have you been working at The Vista School?}

$\square 1$

$\square 2$

$\square 3$

$\square 4$

$\square 5$

$\square 6$

$\square 7$

$\square 8$

$\square 9$

$\square 10$

$\square 11$

\section{Overall, how satisfied are you with CLM?}

$\square$ Strongly Satisfied

$\square$ Satisfied

$\square$ Somewhat Satisfied

$\square$ Neutral 
$\square$ Somewhat Dissatisfied

$\square$ Dissatisfied

$\square$ Strongly Dissatisfied

\section{The CLM material is easy to use.}

$\square$ Strongly Agree

$\square$ Agree

$\square$ Somewhat Agree

$\square$ Neutral

$\square$ Somewhat Disagree

$\square$ Disagree

$\square$ Strongly Disagree

7. I feel well supervised by couch when I teach students using the CLM curriculum.

$\square$ Strongly Agree

$\square$ Agree

$\square$ Somewhat Agree

$\square$ Neutral

$\square$ Somewhat Disagree

$\square$ Disagree

$\square$ Strongly Disagree 
8. I feel I was well trained by couch to use the CLM Curriculum before I began using it with my students.

$\square$ Strongly Agree

$\square$ Agree

$\square$ Somewhat Agree

$\square$ Neutral

$\square$ Somewhat Disagree

$\square$ Disagree

$\square$ Strongly Disagree

9. I feel I have received enough information from coach about the key components of the CLM and how I may use them in my classroom.

$\square$ Strongly Agree

$\square$ Agree

$\square$ Somewhat Agree

$\square$ Neutral

$\square$ Somewhat Disagree

$\square$ Disagree

$\square$ Strongly Disagree 
10. I feel I have received enough information from coach on what the CLRA is and how to use it in my classroom.

$\square$ Strongly Agree

$\square$ Agree

$\square$ Somewhat Agree

$\square$ Neutral

$\square$ Somewhat Disagree

$\square$ Disagree

$\square$ Strongly Disagree

\section{I feel the CLRA is "user-friendly."}

$\square$ Strongly Agree

$\square$ Agree

$\square$ Somewhat Agree

$\square$ Neutral

$\square$ Somewhat Disagree

$\square$ Disagree

$\square$ Strongly Disagree 
12. I feel that I can quickly obtain assistance (who to call or where to go) with difficult student behavior.
$\square$ Strongly Agree
$\square$ Agree
$\square$ Somewhat Agree
$\square$ Neutral
$\square$ Somewhat Disagree
$\square$ Disagree
$\square$ Strongly Disagree

13. I am aware that the CLM for children with Autism is developing specific behavioral repertoires.

$\square$ Strongly Agree

$\square$ Agree

$\square$ Somewhat Agree

$\square$ Neutral

$\square$ Somewhat Disagree

$\square$ Disagree

$\square$ Strongly Disagree 
14. I believe that the CLM has been effective in developing Writer Repertoires for ALL of my students.
$\square$ Strongly Agree
$\square$ Agree
$\square$ Somewhat Agree
$\square$ Neutral
$\square$ Somewhat Disagree
$\square$ Disagree
$\square$ Strongly Disagree
1. $\square$ Strongly Agree
2. $\square$ Agree
3. $\square$ Somewhat Agree
4. $\square$ Neutral
5. $\square$ Somewhat Disagree
6. $\square$ Disagree
7. $\square$ Strongly Disagree

15. I believe that the CLM has been effective in developing Writer Repertoires for SOME of my students.

16. I believe that the CLM has been effective in developing Listener Repertoires for ALL of my students.

$\square$ Strongly Agree

$\square$ Agree

$\square$ Somewhat Agree

$\square$ Neutral 
$\square$ Somewhat Disagree

$\square$ Disagree

$\square$ Strongly Disagree

17. I believe that the CLM has been effective in developing Listener Repertoires for SOME of my students.

8. $\square$ Strongly Agree

9. $\square$ Agree

10. $\square$ Somewhat Agree

11. $\square$ Neutral

12. $\square$ Somewhat Disagree

13. $\square$ Disagree

14. $\square$ Strongly Disagree

18. I believe that the CLM has been effective in developing Problem Solver Repertoires for $\underline{A L L}$ of my students.

$\square$ Strongly Agree

$\square$ Agree

$\square$ Somewhat Agree

$\square$ Neutral

$\square$ Somewhat Disagree

$\square$ Disagree

$\square$ Strongly Disagree 
19. I believe that the CLM has been effective in developing Problem Solver Repertoires for SOME of my students.

$\square$ Strongly Agree

$\square$ Agree

$\square$ Somewhat Agree

$\square$ Neutral

$\square$ Somewhat Disagree

$\square$ Disagree

$\square$ Strongly Disagree

20. I believe that the CLM has been effective in developing Reader Repertoires for ALL of my students.

$\square$ Strongly Agree

$\square$ Agree

$\square$ Somewhat Agree

$\square$ Neutral

$\square$ Somewhat Disagree

$\square$ Disagree

$\square$ Strongly Disagree 
21. I believe that the CLM has been effective in developing Reader Repertoires for SOME of my students.

$\square$ Strongly Agree

$\square$ Agree

$\square$ Somewhat Agree

$\square$ Neutral

$\square$ Somewhat Disagree

$\square$ Disagree

$\square$ Strongly Disagree

22. I believe that the CLM has been effective in developing Observer Repertoires for ALL of my students.

$\square$ Strongly Agree

$\square$ Agree

$\square$ Somewhat Agree

$\square$ Neutral

$\square$ Somewhat Disagree

$\square$ Disagree

$\square$ Strongly Disagree

23. I believe that the CLM has been effective in developing Observer Repertoires for SOME of my students.

$\square$ Strongly Agree

$\square$ Agree

$\square$ Somewhat Agree 
$\square$ Neutral

$\square$ Somewhat Disagree

$\square$ Disagree

$\square$ Strongly Disagree

24. I believe that the CLM has been effective in developing Talker Repertoires for ALL of my students.

$\square$ Strongly Agree

$\square$ Agree

$\square$ Somewhat Agree

$\square$ Neutral

$\square$ Somewhat Disagree

$\square$ Disagree

$\square$ Strongly Disagree

25. I believe that the CLM has been effective in developing Talker Repertoires for SOME of my students.

$\square$ Strongly Agree

$\square$ Agree

$\square$ Somewhat Agree

$\square$ Neutral

$\square$ Somewhat Disagree

$\square$ Disagree

$\square$ Strongly Disagree 
26. I think CLM has been effective in developing Participator Repertoires for ALL of my students.

\author{
$\square$ Strongly Agree \\ $\square$ Agree \\ $\square$ Somewhat Agree \\ $\square$ Neutral \\ $\square$ Somewhat Disagree \\ $\square$ Disagree \\ $\square$ Strongly Disagree
}

27. I think CLM has been effective in developing Participator Repertoires for SOME of my students.

28. $\square$ Strongly Agree

29. $\square$ Agree

30. $\square$ Somewhat Agree

31. $\square$ Neutral

32. $\square$ Somewhat Disagree

33. $\square$ Disagree

34. $\square$ Strongly Disagree

35. I feel comfortable using the CLM lessons plans.

$\square$ Strongly Agree

$\square$ Agree

$\square$ Somewhat Agree 
$\square$ Neutral

$\square$ Somewhat Disagree

$\square$ Disagree

$\square$ Strongly Disagree

36. I feel that CLM coaches are helpful and supportive.

$\square$ Strongly Agree

$\square$ Agree

$\square$ Somewhat Agree

$\square$ Neutral

$\square$ Somewhat Disagree

$\square$ Disagree

$\square$ Strongly Disagree

37. I am frequently using the CLM support materials in my classroom.

$\square$ Strongly Agree

$\square$ Agree

$\square$ Somewhat Agree

$\square$ Neutral

$\square$ Somewhat Disagree

$\square$ Disagree

$\square$ Strongly Disagree 
38. Pleases help us understand in what ways the CLM can be improved. Tells us what you would like to change?

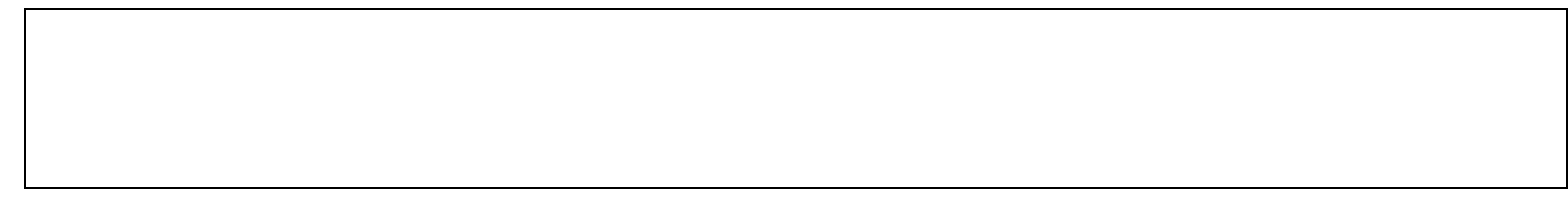

39. What do you think is the strongest component of the CLM? 


\section{Outcomes Phase}

Assessment of Actual Capacity of CLM 


\section{Outcome Phase}

Objective: To determinate the actual capacity (impact) of the intervention the degree, form, and speed of the changes in Autism behavior) via (Vineland -II) and (PLSTM-5)

Administration and Data Analyses of Vineland -II) and (PLS $\left.{ }^{\text {TM}}-5\right)$

\begin{tabular}{|c|c|c|}
\hline ITEM & VINELAND - II & $\begin{array}{l}\text { DATA ANALYSIS } \\
\end{array}$ \\
\hline 1 & Adaptive behavior & \multirow{4}{*}{$\begin{array}{l}\text { Descriptive Statistics } \\
\text { - } \quad \text { Means } \\
\text { - } \quad \text { Standard Deviations } \\
\text { - } \\
\text { Inferential Statistics } \\
\text { - } \quad \text { Dependent } t-\text { test }\end{array}$} \\
\hline 2 & Receptive Language & \\
\hline 3 & Expressive language & \\
\hline 4 & Personal & \\
\hline 5 & Domestic & \multirow{4}{*}{$\begin{array}{l}t=\frac{\frac{\Sigma d}{N}}{\sqrt{\frac{\sum d^{2}-\frac{(\Sigma d)^{2}}{N}}{N(N-1)}}} \\
\begin{array}{l}\text { Kolmogorov-Smirnov Test } \\
\text { Levene's Test }\end{array}\end{array}$} \\
\hline 6 & Intommononol Dolotion & \\
\hline 7 & Interpersonal Relationship & \\
\hline 8 & Leisure time & \\
\hline 10 & Coping Skills & [[Graphics (Scatterplot) \\
\hline
\end{tabular}




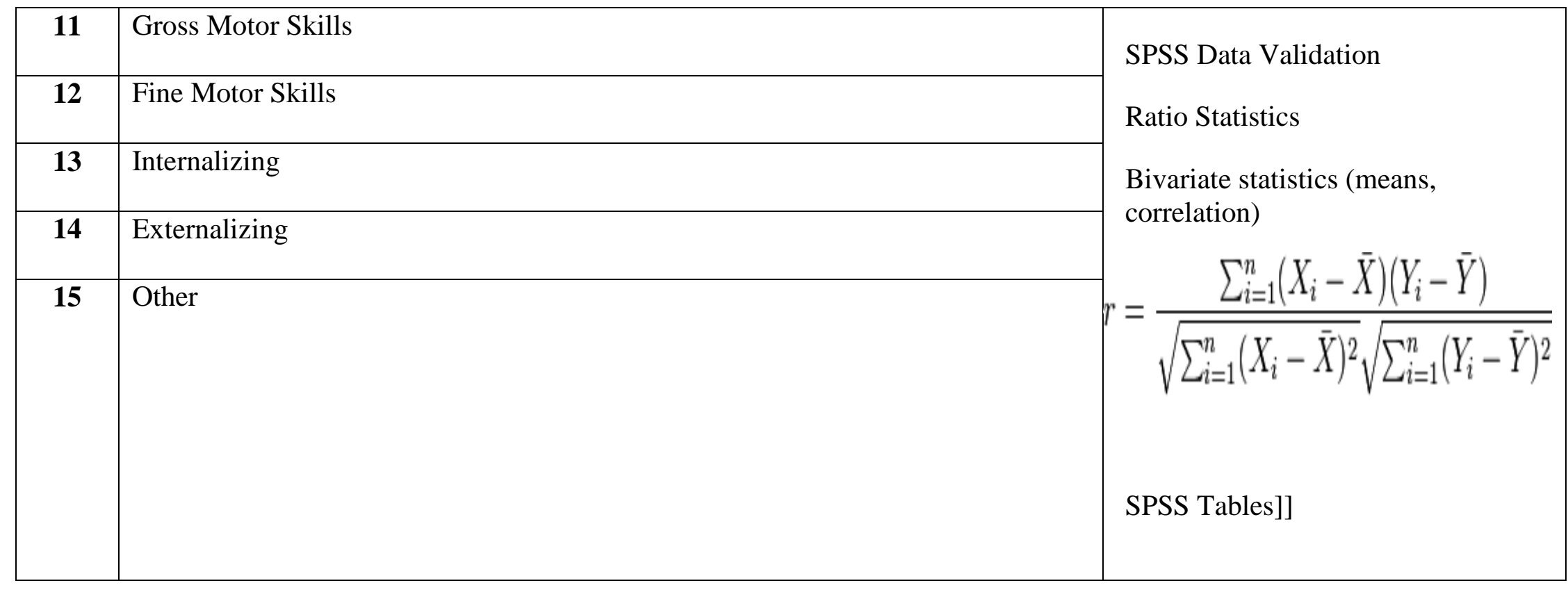

\begin{tabular}{|c|l|l|}
\hline ITEM & \multicolumn{1}{|c|}{ PLS $^{\text {TM-5 }}$} & \multicolumn{1}{c|}{ DATA ANALYSIS } \\
\hline $\mathbf{1}$ & Auditory Comprehension & $\begin{array}{l}\text { Descriptive Statistics } \\
\text { Means } \\
\end{array}$ \\
$\mathbf{2}$ & Expressive Communication & $\begin{array}{l}\text { Standard Deviations } \\
\text { Inferential Statistics } \\
\text { Dependent } t-\text { test }\end{array}$ \\
\hline
\end{tabular}




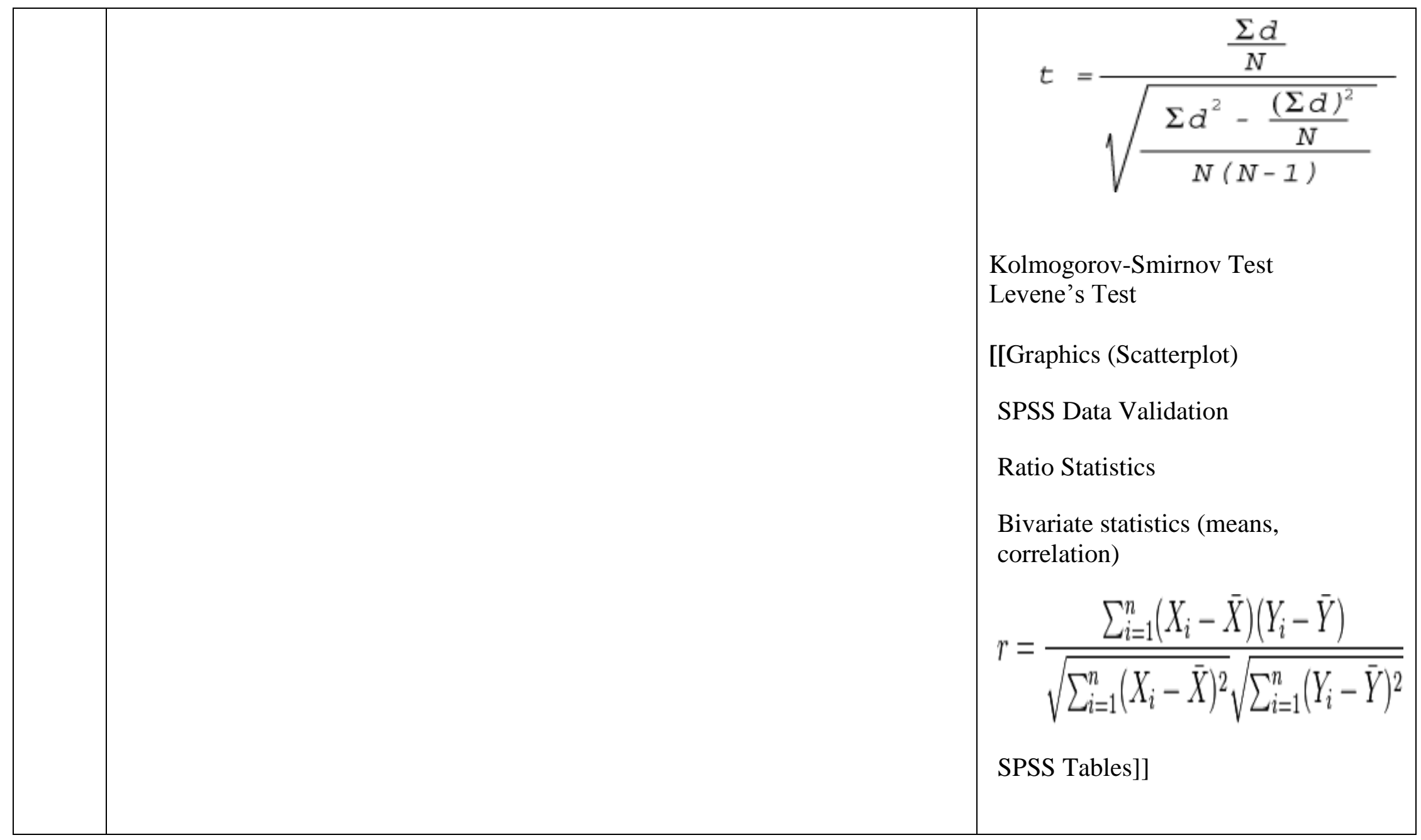




\section{Understanding Phase \\ Assessment of Actual Capacity of CLM}




\section{Understanding Phase}

Results and interpretation of the evaluation to:

Expand our knowledge about the Competent Learned Model

$>$ Provide a contextual understanding of effectiveness of the Competent Learned Model

$>$ Identify who benefits the most from the Competent Learned Model

$>$ Identify which children can benefit the most from what structures of the Competent Learned Model

$>$ Autism Symptoms Distribution [tabulation of symptoms, their intensity and frequency] at the Vista School

Dynamics of Autism symptomology [the relationships among symptoms] at the Vista School 